


\title{
Modern Applications of Electrostatics and Dielectrics
}

\author{
Edited by Dengming Xiao \\ and Krishnaswamy Sankaran
}



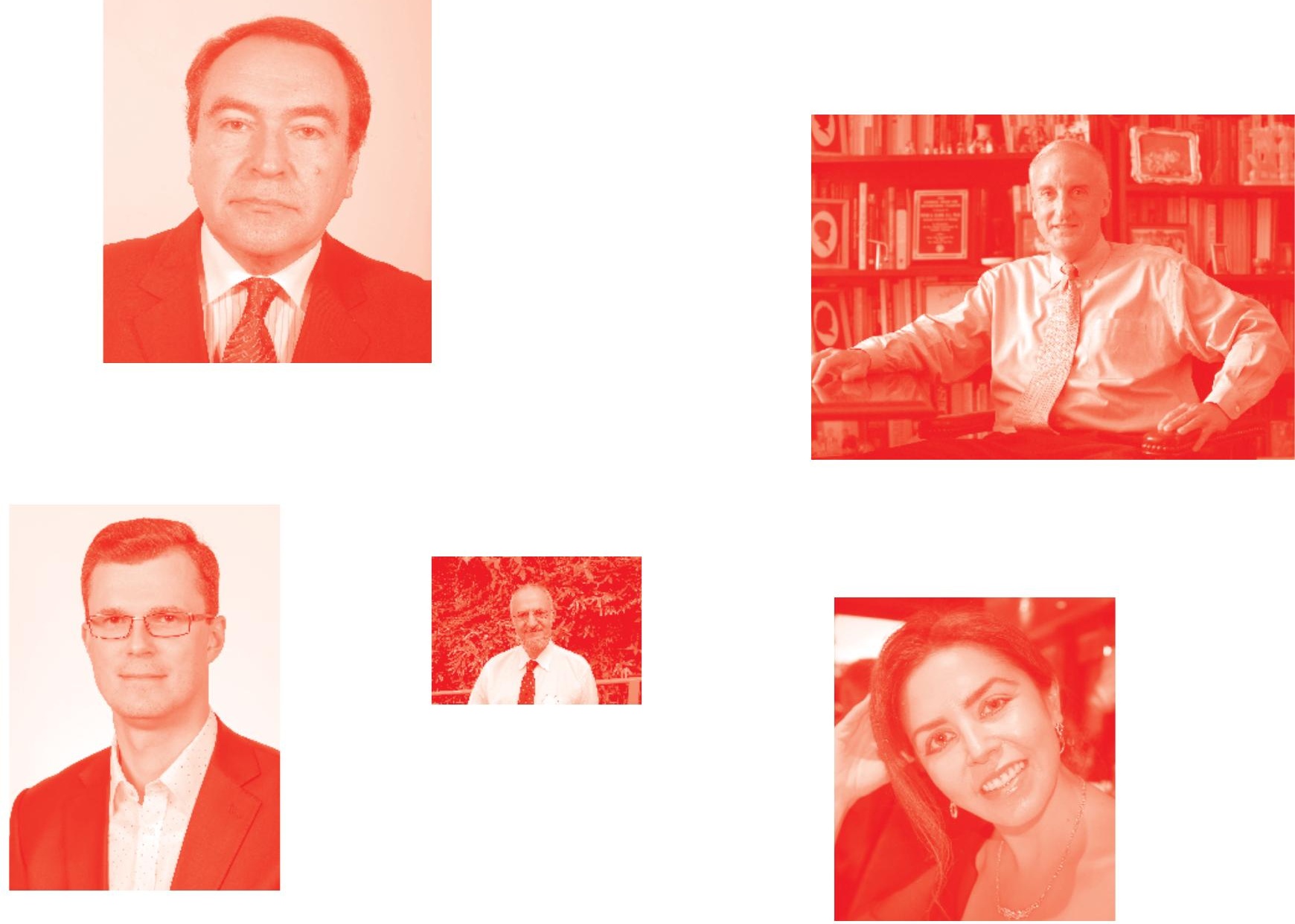

Supporting open minds since 2005
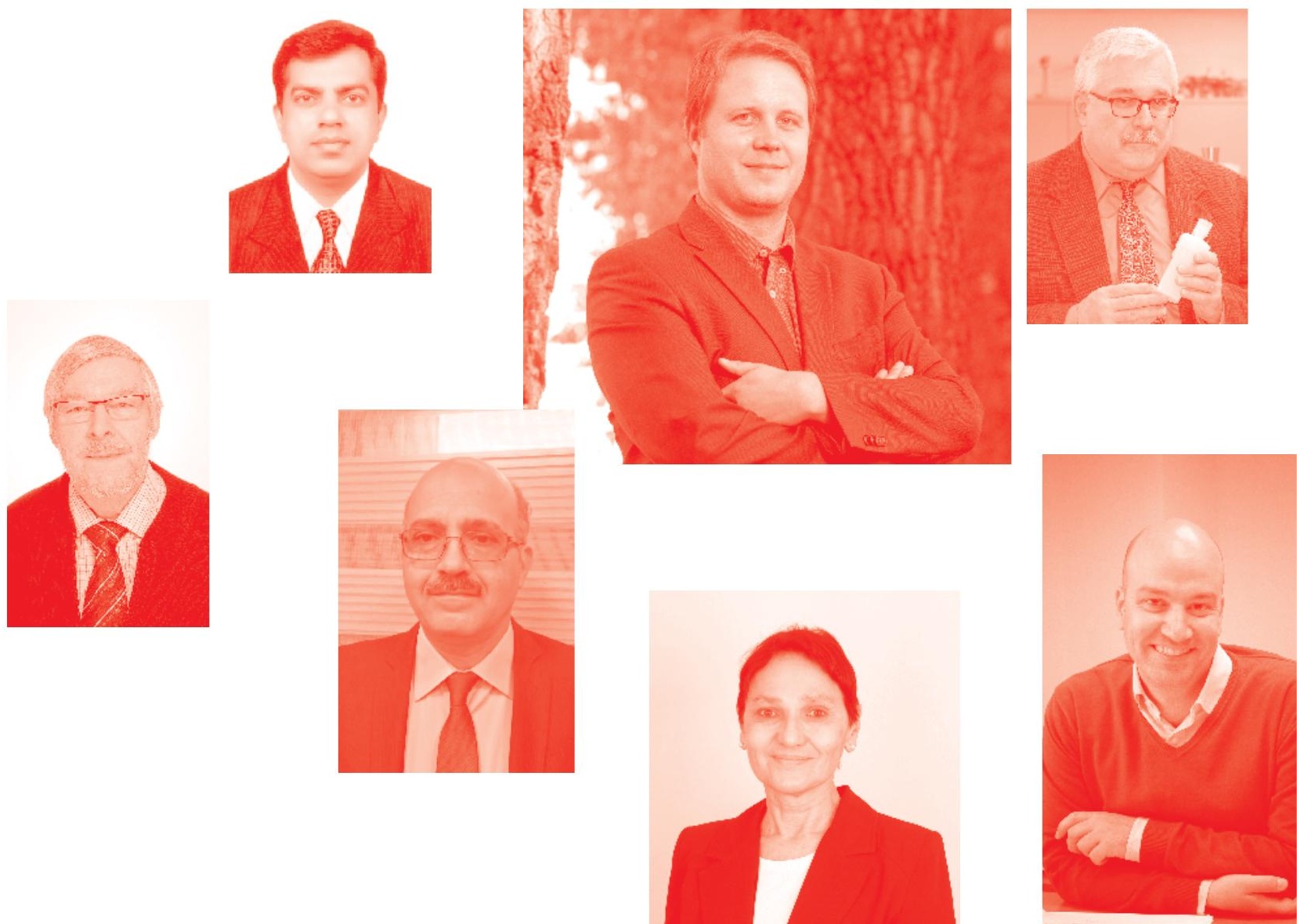
Modern Applications of Electrostatics and Dielectrics

http : //dx. doi . org/10.5772/intechopen. 78806

Edited by Dengming Xiao and Krishnaswamy Sankaran

\section{Contributors}

Chao Wang, Katsuyuki Takahashi, Isao Hiyoshi, Yosuke Enomoto, Shinichi Yamaguchi, Hidemi Nagata, Koichi Takaki, Reza Haghighi Osgouei, Yan Yang, Guangning Wu, Muhammad Mukhlisin, Almushfi Saputra, Zion Menachem, Su Zhao, Dengming Xiao, Krishnaswamy Sankaran, Yifan Kang, Yonglin Bai

( ) The Editor(s) and the Author(s) 2020

The rights of the editor(s) and the author(s) have been asserted in accordance with the Copyright, Designs and Patents Act 1988. All rights to the book as a whole are reserved by INTECHOPEN LIMITED. The book as a whole (compilation) cannot be reproduced, distributed or used for commercial or non-commercial purposes without INTECHOPEN LIMITED's written permission. Enquiries concerning the use of the book should be directed to INTECHOPEN LIMITED rights and permissions department (permissions@intechopen.com).

Violations are liable to prosecution under the governing Copyright Law .

\section{(cc) BY}

Individual chapters of this publication are distributed under the terms of the Creative Commons Attribution 3.๑ Unported License which permits commercial use, distribution and reproduction of the individual chapters, provided the original author(s) and source publication are appropriately acknowledged. If so indicated, certain images may not be included under the Creative Commons license. In such cases users will need to obtain permission from the license holder to reproduce the material. More details and guidelines concerning content reuse and adaptation can be found at http : //www . intechopen . com/copyright-policy . html .

\section{Notice}

Statements and opinions expressed in the chapters are these of the individual contributors and not necessarily those of the editors or publisher. No responsibility is accepted for the accuracy of information contained in the published chapters. The publisher assumes no responsibility for any damage or injury to persons or property arising out of the use of any materials, instructions, methods or ideas contained in the book.

First published in London, United Kingdom, 2020 by IntechOpen IntechOpen is the global imprint of INTECHOPEN LIMITED, registered in England and Wales, registration number: 11086078 , 7th floor, 10 Lower Thames Street, London,

EC3R 6AF, United Kingdom

Printed in Croatia

British Library Cataloguing-in-Publication Data

A catalogue record for this book is available from the British Library

Additional hard and PDF copies can be obtained from orders@intechopen.com

Modern Applications of Electrostatics and Dielectrics

Edited by Dengming Xiao and Krishnaswamy Sankaran

p. cm.

Print ISBN 978-1-83880-301-8

Online ISBN 978-1-83880-302-5

eBook (PDF) ISBN 978-1-83962-984-6 


\section{We are IntechOpen, \\ the world's leading publisher of Open Access books}

\section{Built by scientists, for scientists}

\section{$4,900+$}

Open access books available

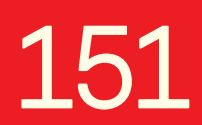

Countries delivered to

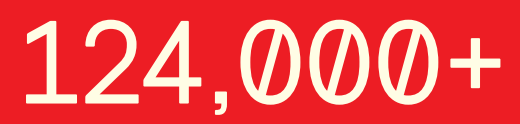

International authors and editors

Our authors are among the

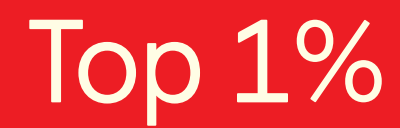

most cited scientists

Contributors from top 500 universities
$140 \mathrm{M}+$

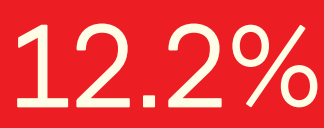

$12.2 \%$

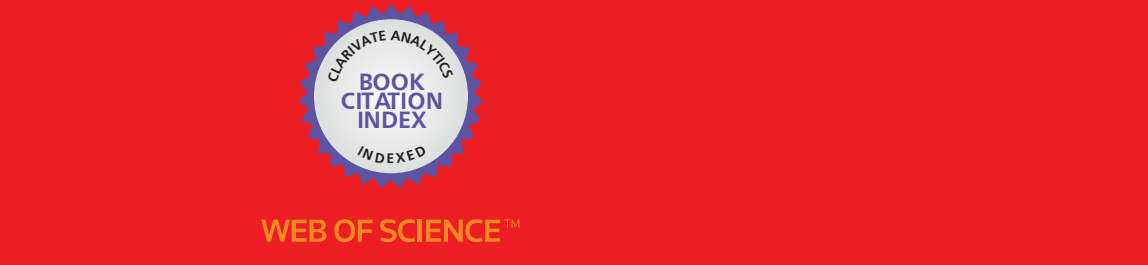

Selection of our books indexed in the Book Citation Index

in Web of Science ${ }^{\mathrm{TM}}$ Core Collection (BKCI)

\section{Interested in publishing with us? \\ Contact book.department@intechopen.com}

Numbers displayed above are based on latest data collected.

For more information visit www.intechopen.com 



\section{Meet the editors}

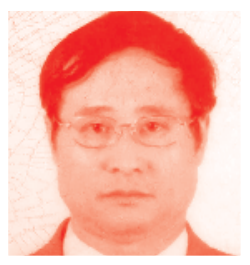

Dengming Xiao was born in Guangxi Province, China in 1953. He received a $\mathrm{PhD}$ from the School of Electrical Engineering, Xian Jiaotong University, China, in 1994. Currently, he is Professor in the Department of Electrical Engineering, Shanghai Jiao Tong University, Shanghai, China. He has worked on gas insulation systems, electrical equipment online monitoring, and fault diagnosis for more than 20 years. His current focus is on environmental friendly substitutes for the insulating medium SF6. He has presided over a number of projects supported by the Natural Science Foundation of China and has published more than 150 papers.

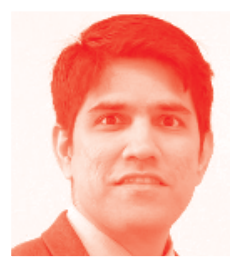

Dr. Krishnaswamy Sankaran is the Chief Executive Officer of Radical Innovations Group AB, a clean energy and circular economy infrastructure development company based in Finland. He is the winner of the 2020 Mission Innovation Champion Award under the flagship of 24 countries around the world plus European Commission for his pioneering work in Clean Energy and Circular Economy. He is an established industrialist and entrepreneur with several years of demonstrated track record in energy, utilities, infrastructure, manufacturing, and recycling industries. He has served in various operational and leadership roles in industries, the World Economic Forum, and the European Commission in more than sixteen countries and four continents. He is nominated by the Government of Finland for the prestigious 2020 Mission Innovation Champion Award, a global recognition for clean energy innovation (http://mission-innovation. net/). Dr. Sankaran received a doctorate in Engineering Sciences from the Swiss Federal Institute of Technology ETH Zurich, Switzerland, a master's degree from Karlsruhe Institute of Technology KIT, Germany, and a joint executive master's degree in Organisational Development and Leadership from Wharton School, Columbia University, INSEAD, and London Business School. He has several years of training in yoga and Advaita Vedanta, an Indian traditional system of philosophy. 



\section{Contents}

Preface

Chapter 1

Introductory Chapter: Modern Applications of Electrostatics

and Dielectrics

by Krishnaswamy Sankaran

Chapter 2

Coulomb Potential Modulation of Atoms by Strong Light Field:

Electrostatic Tunneling Ionization and Isolated Attosecond Light Pulse

by Chao Wang, Yifan Kang and Yonglin Bai

Chapter 3

Development of a Corona Discharge Ionizer Utilizing High-Voltage

AC Power Supply Driven by PWM Inverter for Highly Efficient

Electrostatic Elimination

by Katsuyuki Takahashi, Koichi Takaki, Isao Hiyoshi, Yosuke Enomoto,

Shinichi Yamaguchi and Hidemi Nagata

Chapter 4

Electrostatic Friction Displays to Enhance Touchscreen Experience

by Reza Haghighi Osgouei

Chapter 5

Failure Mechanism and Property Modification of Insulation Material Used in Inverter-Fed Motors

by Yan Yang and Guangning Wu

Chapter 6

Dielectric Analysis Model for Measurement of Soil Moisture Water Content Using Electrical Capacitance Volume Tomography

by Mukhlisin Muhammad and Saputra Almushfi

Chapter 7

Techniques for Calculating Two Interesting Types of Dielectric Materials in Straight Rectangular Waveguides and Their Applications

by Zion Menachem

Research Progress on Synergistic Effect between Insulation Gas Mixtures by Su Zhao and Dengming Xiao 



\section{Preface}

Electrostatics and dielectrics have important applications in modern society. The research on electrostatics and dielectrics has been very active, and the emergence and development of modern science and technology has made a great contribution to the in-depth study of various dielectric materials and electrostatics. This research has played a great role in promoting the further production and development of science and technology. However, with the continuous development of modern society, better characteristic requirements have been put forward for dielectric materials and electrostatics. More and more equipment needs to operate at high frequency, high voltage, high temperature, and other harsh conditions. Therefore dielectric materials need high performance requirements as well as good work stability. This book contains the research progress and application status of electrostatics and dielectrics in different fields.

There are eight chapters in the book. The first chapter is the introductory chapter of modern applications of electrostatics and dielectrics. The second chapter is about the electrostatic potential modulation of atoms. The third chapter examines the development of a new electrostatic eliminator using a pulse width modulation (PWM) inverter. The fourth chapter discusses the electrostatic effect application of touch screens. The fifth chapter analyzes the variable frequency motor insulation failure mechanism and improvement measures. The sixth chapter is about the dielectric analyzing model of soil moisture content. The seventh chapter discusses dielectric materials in the study of the calculation method of the rectangular waveguide. Finally, the last chapter is about the study of synergistic effect of mixed-gas dielectrics.

Dengming Xiao Shanghai Jiao Tong University,

China

Krishnaswamy Sankaran

Radical Innovations Group - RIG,

Finland 



\section{Introductory Chapter: Modern Applications of Electrostatics and Dielectrics}

Krishnaswamy Sankaran

\section{Introduction}

Electrostatics is one of the most basic, yet very important, branches of physics with applications in almost every domain. We observe how a plastic comb after its use on dry hair attracts small bits of paper. We are stunned by the power of lightning from dark rainy clouds during thunder storms. Time to time, we might have also experienced small electric shocks when we touch any metal after rubbing hands on certain materials. All these experiences have to do with the underlying phenomena of electrostatics.

Materials are broadly classified into two large groups based on their electrical properties. Some are called conductors and the others are termed as insulators (or dielectrics). In dielectrics, contrary to metals, all charges are attached to specific atoms and molecules. Such charges are known as bound charges. These charges can be displaced within an atom or a molecule. Compared to the dramatic rearrangements of charges in a conductor under electric field, charge displacements in dielectric materials are only microscopic in nature. However, these microscopic displacements can have cumulative effects that are responsible for several characteristic behaviours of dielectric materials.

Electrostatics-based applications are ubiquitous. Lightning conductors, surface coaters, electrostatic imagers, non-impact printers, industrial processes such as material separation, electrodialysis, static dischargers, etc., are some of the most common applications [1-5].

Dielectrics have been used for developing various devices particularly using their unique material properties. Recent advancements in material science have made it possible to develop and engineer devices for applications in elasto-optics, electro-optics, ultrasonic [6] and surface-mount electronics [7]. Dielectrics are also used to develop compact and efficient antennas [8]. There is an increase in the use of dielectrics for developing advanced defence applications [9]. Dielectric material properties can carefully engineered to develop artificial materials and coatings that can efficiently absorb or dampen incident electromagnetic waves. Such materials are both used in real-time stealth applications $[10,11]$ and modelling absorbing layers to truncate computational domains [12].

Accurate modelling of dielectric properties is one of the most challenging tasks. For basic applications like studying oil spills on ocean surface, one can easily model oil spills as dielectric with complex permittivity [13]. Other advanced applications require more detailed modelling of dielectric properties. For example, dielectric elastomers are actively studied for applications involving artificial muscle actuators for robots and solid-state optical devices for various electronic components [14]. 
These advanced applications of electrostatics and dielectrics rely on accurate modelling methods and tools. The choice of right electromagnetic tools for modelling is an important challenge for design and application engineers $[15,16]$. Various advanced modelling tools are being developed for modelling such applications that includes some of the classical computational methods [17, 18], and non-mainstream computational methods $[19,20]$.

In this book, we have introduced seven advanced applications of electrostatics and dielectrics. These papers bring forth recent developments using electrostatics and dielectrics. In the first paper on electrostatic potential modulation of atoms by strong light field, Wang presents tunnelling ionization and single isolated attosecond light pulse application. In the second paper, Takahashi et al. discuss development of an electrostatic eliminator utilizing high-voltage AC power supply-driven by pulse width modulation (PWM) inverter. In the third paper, Osgouei shows how electrostatic friction mechanism can be used in display technology to enhance touchscreen experience. In the fourth paper, Wu and Yang investigate dielectric failure mechanism and property modification in inverter-fed motors. Mukhlisin and Saputra elaborate their dielectric analysis model for measuring soil moisture (water content) using electric capacitance volume tomography in the fifth paper. Menachem presents techniques for calculating dielectric maternal parameters for waveguide applications in the sixth paper. In the final paper of this book, Xiao and Zhao discuss research progress on synergistic effect between insulation gas mixtures.

I believe this compendium of research papers in the domain of electrostatics and dielectrics will open new opportunities for future research. I thank all the authors for their collaborative efforts and excellent contribution. Final words of gratitude are due to IntechOpen for making this work possible. Good luck!

\section{Author details}

Krishnaswamy Sankaran

Radical Innovations Group AB, Vaasa, Finland

*Address all correspondence to: krish@sankaran.org

IntechOpen

(C) 2020 The Author(s). Licensee IntechOpen. This chapter is distributed under the terms of the Creative Commons Attribution License (http://creativecommons.org/licenses/ by/3.0), which permits unrestricted use, distribution, and reproduction in any medium, provided the original work is properly cited. (cc) BY 


\section{References}

[1] Bright AW. Modern electrostatics. Physics Education. 1974;9(6):381. DOI: $10.1088 / 0031-9120 / 9 / 6 / 303$

[2] Sharp KA, Honig B. Electrostatic interactions in macromolecules: Theory and applications. Annual Review of Biophysics and Biophysical Chemistry. 1990;19(1):301-332. DOI: 10.1146/ annurev.bb.19.060190.001505

[3] Haga K, Chang JS, Kelly AJ, Crowley JM. Applications of the electrostatic separation technique. In: Handbook of Electrostatic Processes. New York: Marcel Dekker; 1995. pp. 365-386

[4] Jeon JU, Higuchi T. Electrostatic suspension of dielectrics. IEEE Transactions on Industrial Electronics. 1998;45(6):938-946. DOI: 10.1109/41.735338

[5] Castle GS. Industrial applications of electrostatics: The past, present and future. Journal of Electrostatics. 2001;51:1-7. DOI: $10.1016 /$ S0304-3886(01)00068-7

[6] Spencer EG, Lenzo PV, Ballman AA. Dielectric materials for electrooptic, elastooptic, and ultrasonic device applications. Proceedings of the IEEE. 1967;55(12):2074-2108. DOI: 10.1109/ PROC.1967.6087

[7] Hennings D, Klee M, Waser R. Advanced dielectrics: Bulk ceramics and thin films. Advanced Materials. 1991;3(7-8):334-340. DOI: 10.1002/ adma.19910030703

[8] Fumeaux C, Almpanis G, Sankaran K, Baumann D, Vahldieck R. Finite-volume time-domain modeling of the mutual coupling between dielectric resonator antennas in array configurations. In: The Second European Conference on Antennas and Propagation (EuCAP). 2007. pp. 1-4. DOI: 10.1049/ic.2007.0909
[9] Sankaran K. Recent trends in computational electromagnetics for defence applications. Defence Science Journal. 2019;69(1):65-73. DOI: 10.14429/dsj.69.13275

[10] Musal HM, Hahn HT. Thin-layer electromagnetic absorber design. IEEE Transactions on magnetics. 1989;25(5):3851-3853. DOI: 10.1109/ 20.42454

[11] Abbas SM, Chandra M, Verma A, Chatterjee R, Goel TC. Complex permittivity and microwave absorption properties of a composite dielectric absorber. Composites Part A: Applied Science and Manufacturing. 2006;37(11):2148-2154. DOI: 10.1016/j. compositesa.2005.11.006

[12] Sankaran K, Kaufmann T, Fumeaux C, Vahldieck R. Different perfectly matched absorbers for conformal time-domain method: A finite-volume time-domain perspective. In: 23rd Annual Review of Progress in Applied Computational Electromagnetics. 2007. pp. 1712-1718

[13] Sankaran K, Fortuny-Guasch J. Radar remote sensing for oil spill classification (optimization for enhanced classification). In: Proceedings of the 12th IEEE Mediterranean Electrotechnical Conference MELECON. 2004. pp. 511-514. DOI: $10.1109 /$ MELCON.2004.1346979

[14] Pelrine R, Sommer-Larsen $P$, Kornbluh RD, Heydt R, Kofod G, Pei Q, et al. Applications of dielectric elastomer actuators. In: Smart Structures and Materials 2001: Electroactive Polymer Actuators and Devices. Vol. 4329. International Society for Optics and Photonics; 2001. pp. 335349. DOI: $10.1117 / 12.432665$

[15] Sankaran K. Are you using the right tools in computational 
electromagnetics? Engineering Reports. 2019;1(3):1-19. DOI: 10.1002/eng2.12041

[16] Aakash, Bhatt A, Sankaran K. Transcending limits: Recent trends \& challenges in computational electromagnetics. In: Proceedings of IEEE-INAE Workshop on Electromagnetics-IIWE. 2018

[17] Yu W, Mittra R. A conformal finite difference time domain technique for modeling curved dielectric surfaces. IEEE Microwave and Wireless Components Letters. 2001;11(1):25-27. DOI: $10.1109 / 7260.905957$

[18] Brosseau C, Beroual A. Computational electromagnetics and the rational design of new dielectric heterostructures. Progress in Materials Science. 2003;48(5):373-456. DOI: 10.1016/S0079-6425(02)00013-0

[19] Sankaran K. Beyond DIV, CURL and GRAD: Modelling electromagnetic problems using algebraic topology. Journal of Electromagnetic Waves and Applications. 2017;31(2):121-149. DOI: 10.1080/09205071.2016.1257397

[20] Aakash, Bhatt A, Sankaran K. How to model electromagnetic problems without using vector calculus and differential equations? IETE Journal of Education. 2018;59(2):85-92. DOI: $10.1080 / 09747338.2018 .1554456$ 


\title{
Coulomb Potential Modulation of Atoms by Strong Light Field: Electrostatic Tunneling Ionization and Isolated Attosecond Light Pulse
}

\author{
Chao Wang, Yifan Kang and Yonglin Bai
}

\begin{abstract}
An attosecond research upsurge has been overwhelmingly rising since the establishment of novel light source-single isolated attosecond laser in extreme ultraviolet/X-ray resulted by strong field high-order harmonics generation (HHG). In this chapter, based on the electrostatic tunneling ionization from Coulomb potential modulation of atoms by strong light field, we scrutinized the intrinsic phase of high-order harmonics and analyzed qualitatively the salient dependence of two mainstream single isolated attosecond pulse generation techniques as polarization gating $(\mathrm{PG})$ and amplitude gating $(\mathrm{AG})$ on carrier-envelope phase (CEP) of femtosecond driving laser. The conclusion is that the optimized CEP corresponding to the highest intensity contrast between the main and sideband attosecond pulses is $\pi / 2$ and 0 for polarization gating and amplitude gating, respectively. Further, an experimental implementation was given in detail to exemplify the tricks for optimum phase-matching process of HHG from the interaction of high-intensity femtosecond laser field with noble gas target. The effects of the relative location between Gaussian-shaped driving femtosecond laser field focus and the gas target source used on the HHG phase matching were studied, and the conclusion found that the expected position of gas target for optimum phase matching is always lying behind the focal point of the driving field used.
\end{abstract}

Keywords: tunneling ionization, high-order harmonics generation, single isolated attosecond pulse, carrier-envelope phase, phase matching, few-cycle femtosecond laser

\section{Introduction}

With the invention in general and realization of the laser in particular by Maiman in 1960, field of optics soon entered the new era of nonlinear optics. In this regime, the optical properties of materials are no longer independent of the intensity of light-as was believed for hundreds of years before-but rather change with light intensity, giving rise to a wealth of new phenomena, effects, and applications. Today, nonlinear optics has entered our everyday life in many ways and has also 
been the basis for numerous new developments in spectroscopy and laser technology. Indeed, from the moment of birth of nonlinear optics, laser physics and nonlinear optics have been intimately related to each other. Within "traditional" nonlinear optics, the absolute changes of the optical properties are tiny if one follows them versus time on a timescale of a cycle of light. This simple fact is the basis of many concepts and approximations of "traditional" nonlinear optics. Over the years, however, lasers have improved in many ways, especially in terms of the accessible peak intensities and in terms of the minimum pulse duration available. These days, over 50 years after the invention of the laser, the shortest optical pulses generated are about one and a half cycles of light in duration. This comes close to the ultimate limit of a single optical cycle [1].

Thanks to the technique of chirped-pulse amplification (CPA) and the availability of CEP stabilized few-cycle laser [2], amplified laser pulses with focused peak intensities in the range of $10^{22} \mathrm{~W} / \mathrm{cm}^{2}$ are available in some laboratories. As a result of this, today's light intensities can lead to substantial or even to extreme changes on the timescale of light, and the range of optics research is now shifting from the stage of perturbative to extreme (or nonperturbative) nonlinear optical mechanism $[3,4]$. As for the latter, one remarkable achievement is the establishment of novel light source-single isolated attosecond ( 1 attosecond[as] $=10^{-18} \mathrm{~s}$ ) laser pulse in extreme ultraviolet or even X-ray electromagnetic spectrum based on strong field HHG [5-7]. Since the fundamental processes of chemistry, biology, and materials science are triggered or mediated by the motion of electrons inside or between atoms, and that the atomic-scale motion of electrons typically unfolds within tens to thousands of attoseconds, breakthroughs in light source science are now opening the door to watching and controlling these hitherto inaccessible microscopic dynamics, opening up a new horizon of science by observing, controlling, and manipulating nature in a new dimension. Consequently, an attosecond research upsurge is overwhelmingly rising from physics, chemistry, and material science to even information processing and other fields [8-10]. What is worth mentioning is that transnational or transregional attosecond science research infrastructures are emerging, such as Extreme Light Infrastructure Attosecond Light Pulse Source (ELI-ALPS) in Szeged, Hungary. Its main objective is to establish a unique attosecond laser facility which provides developers and users with light sources within the THz to X-ray frequency range in the form of ultrashort pulses at high repetition rate.

Until now, the HHG based on the interaction between high-intensity few-cycle femtosecond laser and noble gases is regarded as the main process for generating attosecond laser pulse. The physical process in essence is frequency up-conversion resulted by optical field caused by atomic tunneling ionization, which means that, no matter what kind of single isolated attosecond pulse generation technique, such as amplitude gating [11] or polarization gating [12], the phase matching involved is the key issue, which affects the macroscopic response of the multiatom gas system and eventually determines the conversion efficiency. According to the three-step scenario of HHG proposed by Corkum [13], phase matching in process of HHG is microcosmically influenced by multiple factors involved: fundamental driving laser field, the gas system used, the high-order harmonics field that generated, and the plasma formed by the atomic ionization. It is very difficult and even impossible to carry out such a complete and accurate analysis on phase matching of HHG. So far, most theoretical analysis are addressing incompletely on one or two factors mentioned above, while the experimental demonstrations are just concerned about high-order harmonics yield, omitting intentionally or unintentionally the important details of phase matching optimization $[14,15]$. Consequently, the experimental details to optimize HHG attosecond laser pulse generation are still not made clear. 
In this chapter, based on the three-step scenario of HHG, we scrutinized the intrinsic phase of high-order harmonics from the atom tunneling ionization induced by strong laser field and analyzed qualitatively the salient dependence of two fundamental single attosecond pulse generation techniques as polarization gating and amplitude gating on CEP of femtosecond driving laser. Finally, an experimental implementation was given in great detail to show the way to realize optimum HHG phase matching during interaction of strong optical field with noble gas target.

\section{Strong field tunneling ionization and HHG}

The light intensities necessary to rapidly ionize an atom are on the order of $\mathrm{I} \approx 10^{14} \sim 10^{16} \mathrm{~W} / \mathrm{cm}^{2}$, which is well within the nonrelativistic regime. Thus, we can ignore the laser magnetic field for the moment and focus on the laser electric field [3]. Figure 1 visualizes this kind of ionization by high-intensity few-cycle femtosecond laser pulse for an electron bound in the Coulomb potential of a nucleus (assumed to be much more massive or fixed in space). The Gaussian linearly polarized laser pulse of $t_{F W H M}=5 f s$ is characterized by its electric field $E(t)=$ $\tilde{E}(t) \cos \left(\omega_{0} t\right)$ with carrier photon energy $\hbar \omega_{0}=1.5 \mathrm{eV}$, together with the twodimensional scheme of the resulting electric potential experienced by an electron initially bound in an atom at three characteristic points in time. The large "tilt" along the electric field vector axis in the center of the pulse can lead to tunneling of the electron out of its binding potential through the potential barrier. If the barrier height is lowered below the binding energy, above-barrier ionization can occur. For circularly polarized light, the "tilt" stays constant, but its axis rotates in time [1].

Whether we can use the concept of electrostatic tunneling for light fields oscillating depends on the ratio between the light field period and the time the electron spends within the barrier, electron tunneling time. If the tunneling time is shorter than the period of light, the laser electric field can indeed be viewed as a static field along its polarization direction that parametrically changes its instantaneous value, which is the case of HHG process-based single attosecond pulse generation. Within the "static-field approximation," the tunneling ionization rate $\Gamma_{i}(t)$ depends exponentially on the instantaneous barrier width $l(t)$ because the electron wave function

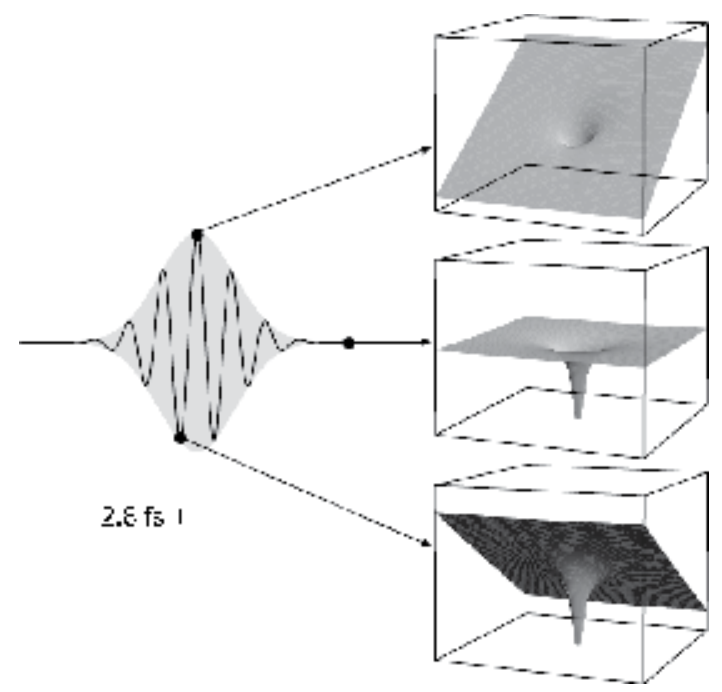

Figure 1.

Coulomb potential modulation of atoms by strong light field. 


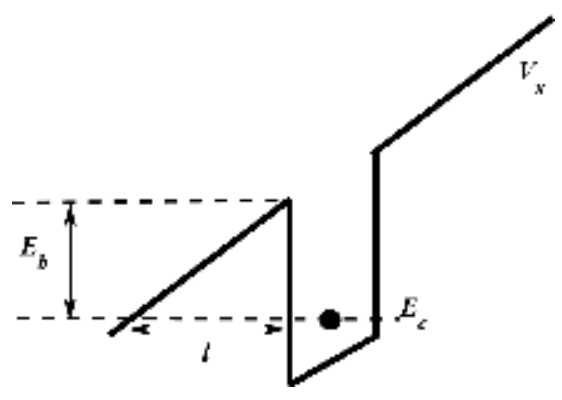

Figure 2.

Strong field-induced tunneling ionization.

is decaying exponentially in the barrier according to $\psi(x) \propto \exp \left(-\left|k_{x}\right| x\right)$, as in

Figure 2. The probability of tunneling through the barrier is $\propto|\psi(l)|^{2}$. Further, one can expect the general behavior $\Gamma_{i}(t)$ as

$$
\Gamma_{i}(t) \propto e^{-\frac{\frac{3}{2}}{E_{b}^{2}} \sqrt{2 m_{e}}} 1(t)
$$

Apparently, the ionization characteristics would definitely have a strong dependence on CEP of the exciting laser pulses.

To get the exact description of the HHG spectrum, one can in principle solve the time-dependent Schrödinger equation numerically for the ionization and compute the atomic dipole moment from the known instantaneous wave function $\Psi(r, t)$ via the expectation value $\langle\Psi(r, t)|-e r| \Psi(\boldsymbol{r}, t)\rangle$. Multiplying by the atoms' density delivers the macroscopic optical polarization $\boldsymbol{P}$, which is one of the main parameters in the Maxwell equations. Neglecting the propagation effects, the radiated electric field is proportional to the second temporal derivative of $\boldsymbol{P}$. The square modulus of its Fourier transform delivers the intensity spectrum of high-order harmonics. The most intuitive way to discuss semiclassically the extreme nonlinear interaction that leads to attosecond pulses is through the so-called three-step scenario introduced by Corkum [13], as in Figure 3. The first step (1) is the atom ionization via tunneling through the distorted Coulomb potential. Now the electron wave packet is released in the continuum where it gets accelerated by the external field (2)). The third step (3) is the possible recombination with the nucleus and emission of a high-energy photon.

As for the second step, the movement of the electron wave packet can be described using Newton's second theorem of classical mechanics. Again, the magnetic field of the laser and the Coulomb potential bound on the electrons are ignored, and only the electric field of the laser is considered. And also we neglect the spatial dependence of the electric field since the wavelength of the laser field is much bigger than the distance the electron moves within the external field. We assume a laser field that the atom is exposed to is $E(t)=E_{0} \cos \left(w_{L} t\right)$. The electron tunnels through the potential wall and is released into the continuum with zero velocity at position zero at birth time $t=t_{i}, v\left(t_{i}\right)=0$, and $x\left(t_{i}\right)=0$. When we assume that the only force experienced by the electron is $F(t)=m_{e} x^{\prime \prime}=-e E(t)$, the expression for $x(t)$ follows by integrating.

$$
\begin{gathered}
v(t)=\frac{e E_{0}}{m_{e} w_{L}}\left[\sin \left(w_{L} t\right)-\sin \left(w_{L} t_{i}\right)\right] \\
x(t)=\frac{e E_{0}}{m_{e} w_{L}^{2}}\left[\cos \left(w_{L} t\right)-\cos \left(w_{L} t_{i}\right)+w_{L}\left(t-t_{i}\right) \sin \left(w_{L} t_{i}\right)\right] .
\end{gathered}
$$


Now one can use Eq. (3) to calculate the return time $t_{r}$ of the electron for different tunneling times $t_{i}$. Since the process is periodic in time with a periodicity of $\pi / w_{L}$, we use $0 \leq t_{i} \leq 0.5 T=\pi / w_{L}$. Using Eq. (3), one can easily get that only those electrons freed at $0 \leq t_{i} \leq 0.25 T$ have the chance to return to the nucleus, as illustrated in Figure 4. Intuitively, the return time $t_{r}$ of electron with birth time $t_{i}$ is the horizontal ordinate of the intersection point formed by the laser field curve and its tangent line passing the point with $t=t_{i}$. And two important values for analyzing HHG are free time $\tau=t_{r}-t_{i}$ that the electron spends in the continuum and the return energy $W_{k i n}$.

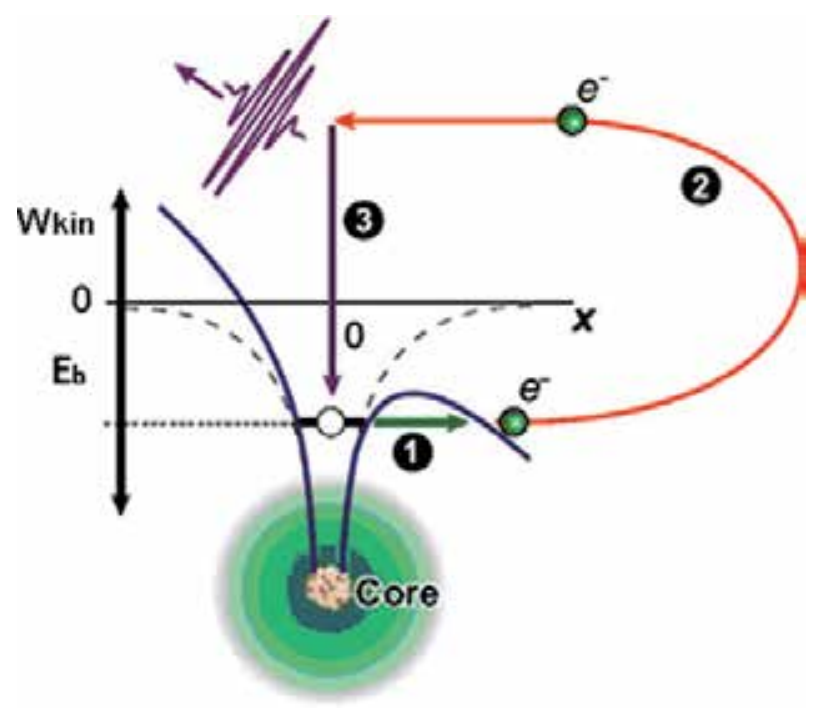

Figure 3 .

Three-step scenario of high-harmonic generation.

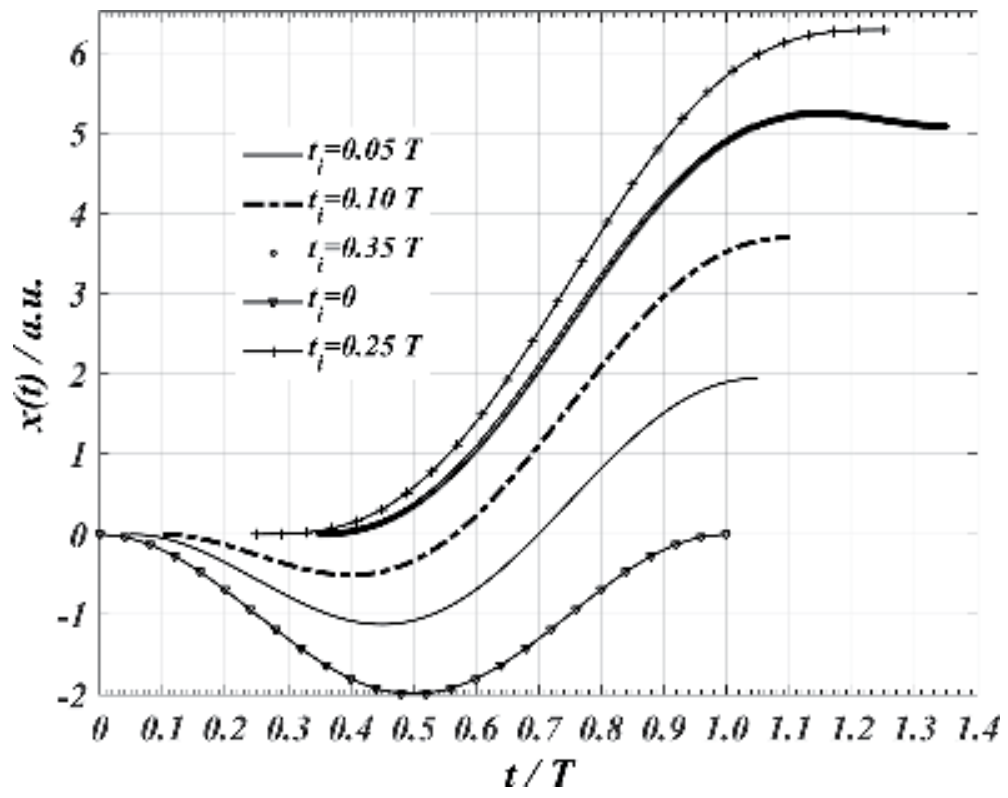

Figure 4 .

Wiggling motion of electrons in external laser field. 


$$
\begin{gathered}
W_{k i n}\left(t_{i}\right)=2 U_{p}\left[\sin \left(w_{L} t_{r}\right)-\sin \left(w_{L} t_{i}\right)\right]^{2} \\
t_{i}=\frac{1}{w_{L}} \operatorname{atan}\left[\frac{-w_{L} \tau+\sin \left(w_{L} \tau\right)}{1-\cos \left(w_{L} \tau\right)}\right] .
\end{gathered}
$$

Here $U_{p}=e^{2} E_{0}^{2} / 4 m_{e} w_{L}^{2}$ is the wiggling electron's ponderomotive energy which is the period-averaged kinetic energy in external laser field $E(t)=E_{0} \cos \left(w_{L} t\right)$.

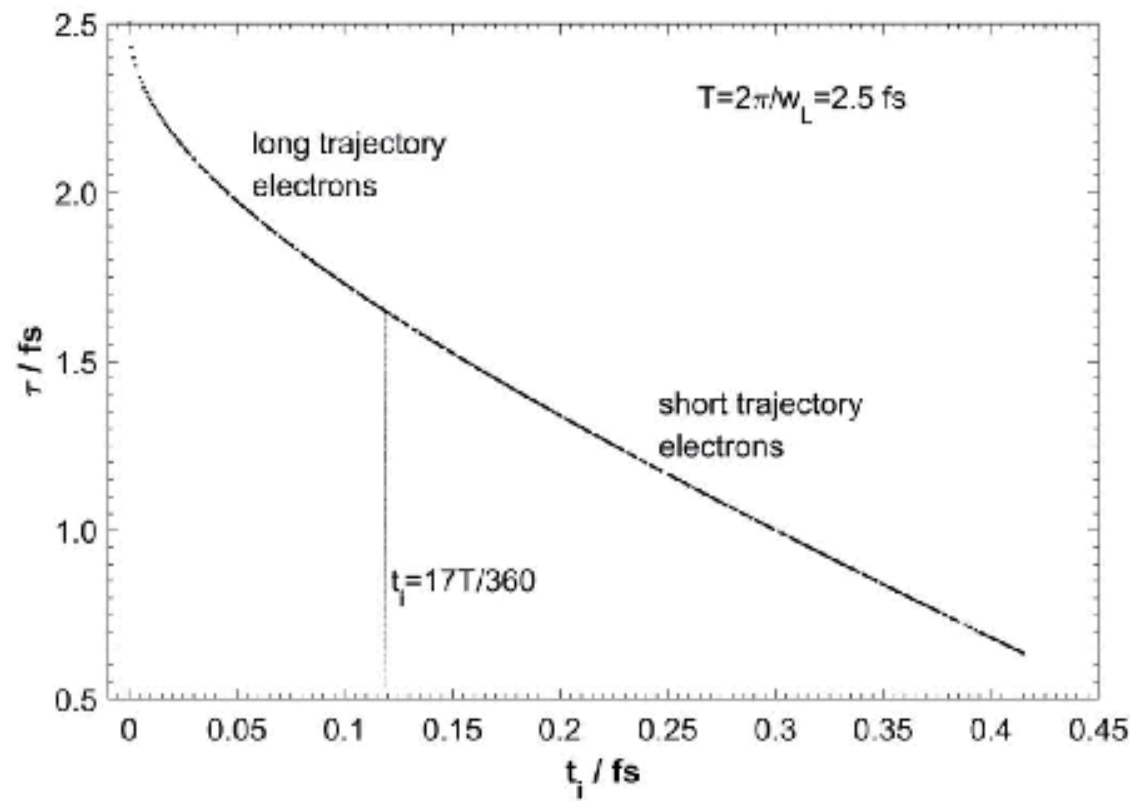

Figure 5.

Electron's free time during the process of HHG.

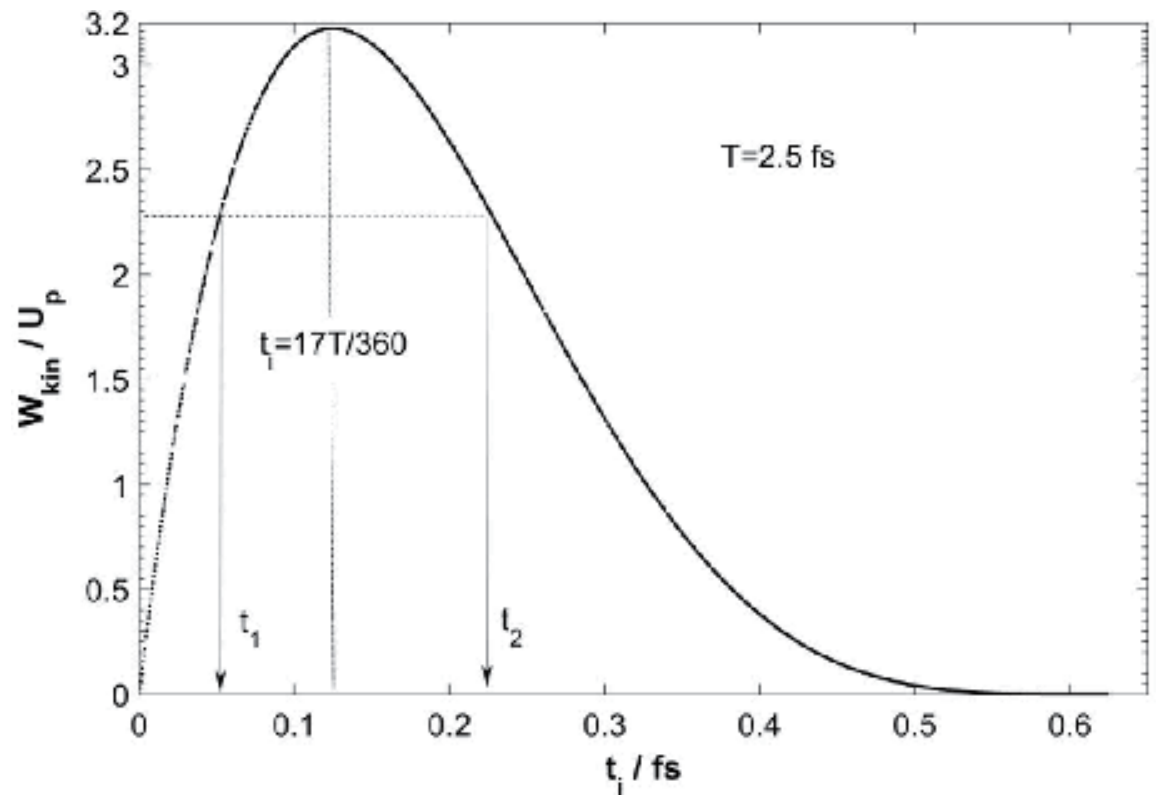

Figure 6.

Electron's return energy during the process of HHG. 
The electrons with different freed time $t_{i}$ are bound to have different free times $\tau$ but might have equal return kinetic energy, as shown in Figures 5 and 6 . Taking the characteristic time $t_{\mathrm{m}}=17 \mathrm{~T} / 360$ as the boundary, which corresponds to the maximum return kinetic energy of $3.17 \mathrm{U}_{\mathrm{p}}$, electrons freed earlier are called long trajectory electrons, while the others short trajectory electrons. The intrinsic phase of high-order harmonics resulted from the latter definitely has linear chirp, since the parameter $\tau$ linearly determining the phase has linear feature.

\section{CEP dependence of single attosecond pulse generation}

Single isolated attosecond pulses originated from HHG process have been demonstrated experimentally by a variety of gating techniques, which include spectral selection of half-cycle cutoffs as in amplitude gating (AG) $[11,16,17]$ and ionization gating (IG) [18-21], temporal gating techniques such as polarization gating (PG) (including double optical gating) [12, 22-27], and spatiotemporal gating with the attosecond lighthouse effect [28, 29]. Based on the intuitive description of HHG as the three-step scenario, PG and AG are the two most fundamental techniques, of which the former utilizes the dependence of HHG yield on driving field ellipticity, while on the latter the essential dependence of HHG itself is on driving field intensity.

PG involves the synthesis of laser field tailored specifically with time-dependent ellipticity. The left and right circularly polarized Gaussian laser fields used are as follows:

$$
\begin{aligned}
& \vec{E}_{L C}(t)=E_{0}\left[e^{-2 \ln (2) \frac{\left(t-T_{d} / 2\right)^{2}}{\tau_{\omega}{ }^{2}}}\right]\left[\cos \left(\frac{2 \pi}{T} t+C E P\right) \hat{x}+\sin \left(\frac{2 \pi}{T} t+C E P\right) \hat{y}\right], \\
& \vec{E}_{R C}(t)=E_{0}\left[e^{-2 \ln (2) \frac{\left(t+T_{d} / 2\right)^{2}}{\tau_{\omega} \omega^{2}}}\right]\left[\cos \left(\frac{2 \pi}{T} t+C E P\right) \hat{x}-\sin \left(\frac{2 \pi}{T} t+C E P\right) \hat{y}\right] .
\end{aligned}
$$

Here $T_{d}$ is the time delay between the two laser pulses. So one can get the field synthesized and its ellipticity:

$$
\begin{aligned}
\vec{E}_{C}(t)= & E_{0}\left[e^{-2 \ln (2) \frac{\left(t-T_{d} / 2\right)^{2}}{\tau_{\omega^{2}}}}+e^{-2 \ln (2) \frac{\left(t+T_{d} / 2\right)^{2}}{\tau_{\omega^{2}}}}\right] \cos \left(\frac{2 \pi}{T} t+C E P\right) \hat{x} \\
& +E_{0}\left[e^{-2 \ln (2) \frac{\left(t-T_{d} / 2\right)^{2}}{\tau_{\omega^{2}}}}-e^{-2 \ln (2) \frac{\left(t+T_{d} / 2\right)^{2}}{\tau_{\omega}{ }^{2}}}\right] \sin \left(\frac{2 \pi}{T} t+C E P\right) \hat{y} \\
\xi(t)= & \frac{\left|1-e^{-4 \ln (2)\left(T_{d} / \tau_{\omega}{ }^{2}\right) t}\right|}{\left|1+e^{-4 \ln (2)\left(T_{d} / \tau_{\omega}{ }^{2}\right) t}\right|} .
\end{aligned}
$$

The field in Eq. (8) can be decomposed into two linearly polarized fields, the driving field and gating field for HHG, respectively. Taking $\tau_{\omega}=2 T=5 f s, T_{d}=$ $5 f s$, and the critical field ellipticity $\xi_{c}=0.2$ [30], one can have the gating zone with notable HHG effect as $T_{G}=1.5 f s$. As shown in Figure 7, the two channels for HHG are appearing different evolutionary characteristics corresponding to the CEP changing from 0 to $\pi / 2$, with channel (1) depressed and channel (2) enhanced. The intensity contrast between the HHG that resulted reaches the maximum at $\mathrm{CEP}=$ $\pi / 2$, meaning the generation of single isolated attosecond pulse from one driving laser pulse. Consequently the optimized phase setting for driving field is CEP $=\pi / 2$. 

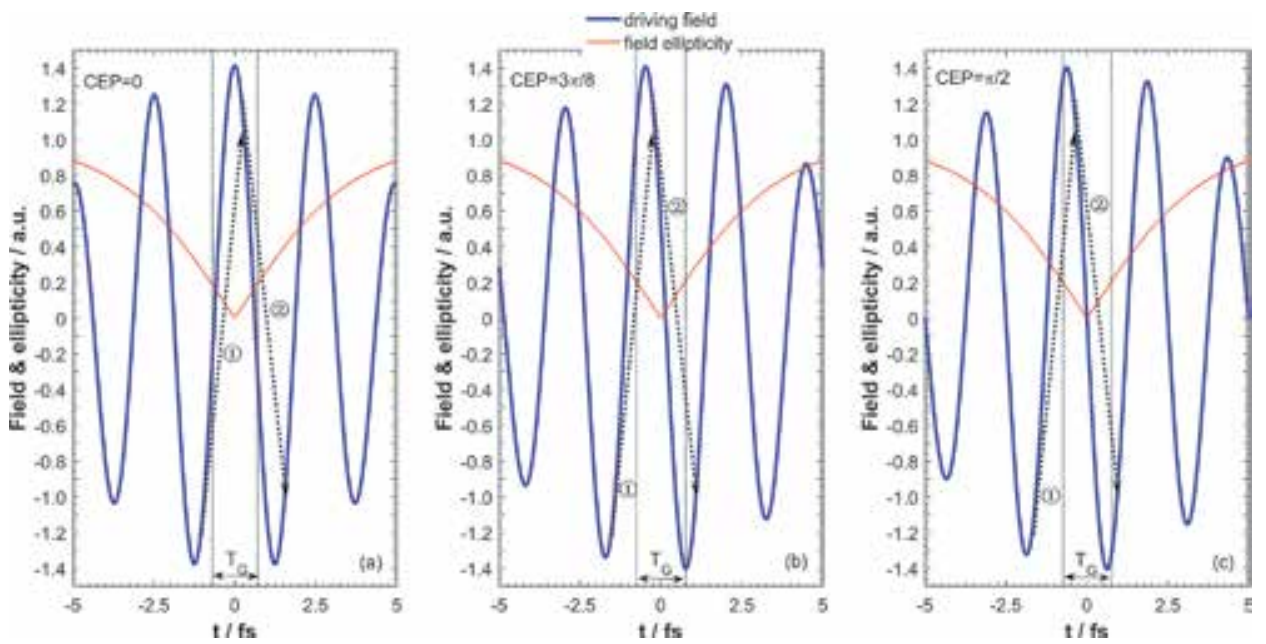

Figure 7.

CEP dependence of $H H G$ for $P G$.
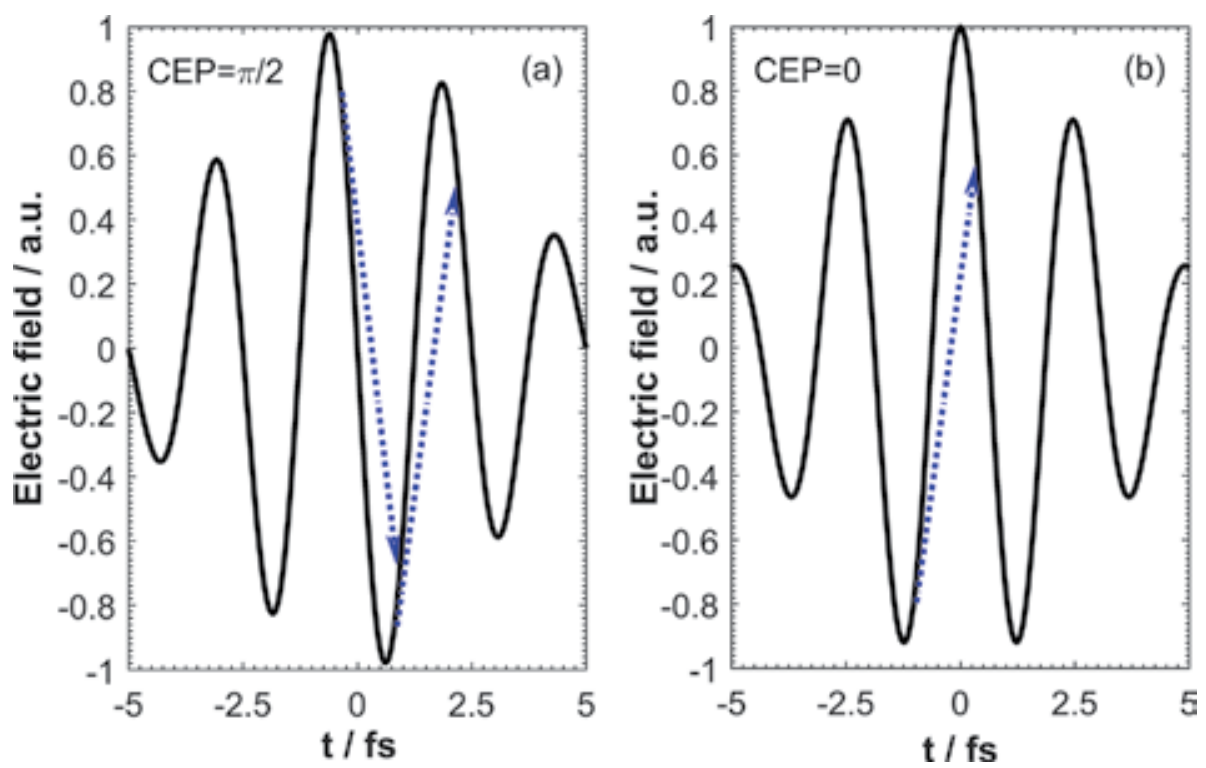

Figure 8.

CEP dependence of $H H G$ for $A G$.

As for AG, the essence is spectral selection of half-cycle HHG cutoffs, so the linearly polarized driving field with CEP $=0$ could guarantee that the cutoff spectrum only comes from one channel and thus is supercontinuum supporting single isolated attosecond pulse, as shown in Figure 8. So the optimized phase setting for AG comparatively is CEP $=0$, which has been proven by the experimental results in Ref. [16].

\section{Phase matching for high-energy attosecond pulse}

The device used for optimizing HHG phase matching is schematically shown in Figure 9. The CEP stabilized few-cycle femtosecond laser (ladled as 1) is used as the 
driving optical field for HHG, with the inert gas (ladled as 5) in the nickel tube (ladled as 4) as the target source. The metal filter (ladled as 8), e.g., aluminum or zirconium, is used to filter the spectrum of residual driving laser from the HHG generated. Acrylic cover (ladled as 12) is specifically adopted for easier monitoring of the driving laser condition when the system is in vacuum. The procedure for optimizing high-order harmonics phase matching includes three steps. The details are deliberated in order as follows.

The first step is done in air. A Ti:sapphire oscillator (Rainbow, Femtolasers $\mathrm{GmbH}$ ) produces sub-10-fs seed pulses with 2.4-nJ energy at 76-MHz. The oscillator was pumped by a continuous wave laser (Coherent Verdi) at 532-nm and 3.10watt. The oscillator output pulses are then stretched before coupling them into the amplifier. The output of the nine-pass amplifier with three-mirror configuration was more than 1.1-mJ with hundreds of picosecond duration and $3-\mathrm{kHz}$ repetition rate. After amplification, the beam is sent into a grating compressor consisting of two high-efficiency transmission gratings and a vertical retro-reflector to get 25 -fs laser pulse of $0.95-\mathrm{mJ}$. The beam from this laser system was focused into a fused silica hollow-core fiber filled with neon gas with a length of 1.2-m (250- $\mu \mathrm{m}$ inner diameter and 750- $\mu \mathrm{m}$ outer diameter) [31]. The broad optical spectrum ranging from 420-nm to around 950-nm obtained by self-phase modulation effect in hollow-core fiber, after traveling through a set of finely designed chirped-mirror compressor, gives laser pulse width of 4.6 -fs which is about 1.7 optical cycles for the center wavelength of 803.5-nm, as shown in Figure 10. With focus spot size of 50$\mu \mathrm{m}$, the peak intensity reaches above the level of $1.5 \times 10^{14} \mathrm{~W} / \mathrm{cm}^{2}$, which is enough to initiate the HHG in inert gas. The high-intensity femtosecond laser pulse will bring about air breakdown plasma near its focus, the color of which is blue and purple, as shown in Figure 11. Under the dark atmosphere, the longitudinal midpoint of the plasma can be roughly regarded as the focus point of the driving pulse. What is worth mentioning is that, before sending the drive pulse to the vacuum chamber, both the nickel tube and the metal filter plates must not be in the propagation path of the driving pulse.

Subsequent experimental steps are done using the focus point determined as a benchmark. Firstly, keep the driving femtosecond laser in its experimental settings, and roughly place the nickel tube $3 \sim 5$-mm after the focus point (yet outside the driving laser path by now to avoid damage). The nickel tube is a hollow-core cylinder with an outer diameter of $2.5-\mathrm{mm}$ and inner diameter of $2.0-\mathrm{mm}$, the top of which is fully sealed to keep the inert gas system. Secondly, lower the power of the driving laser and then move slowly the nickel tube into the laser path so that the

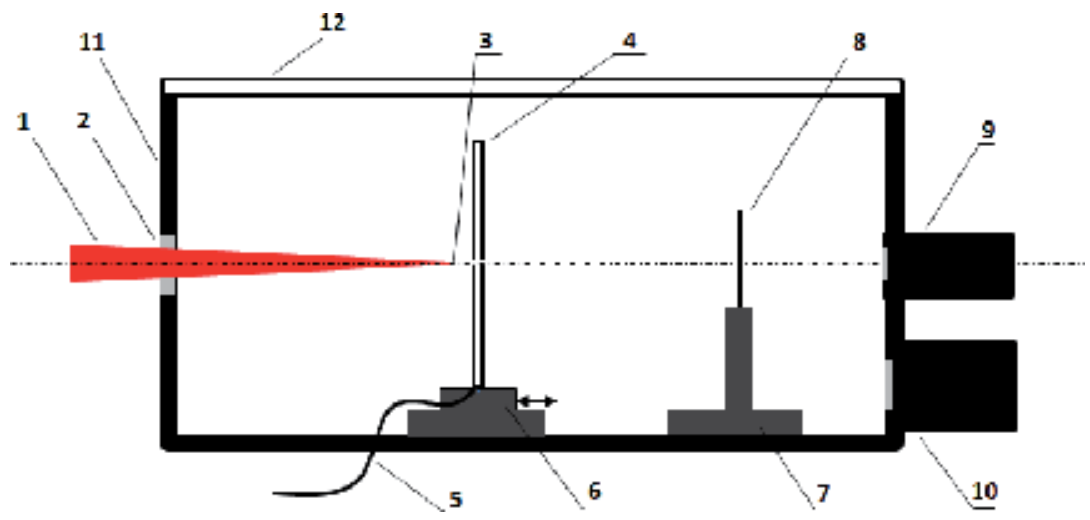

Figure 9.

Schematic diagram of the HHG experimental illustration. 


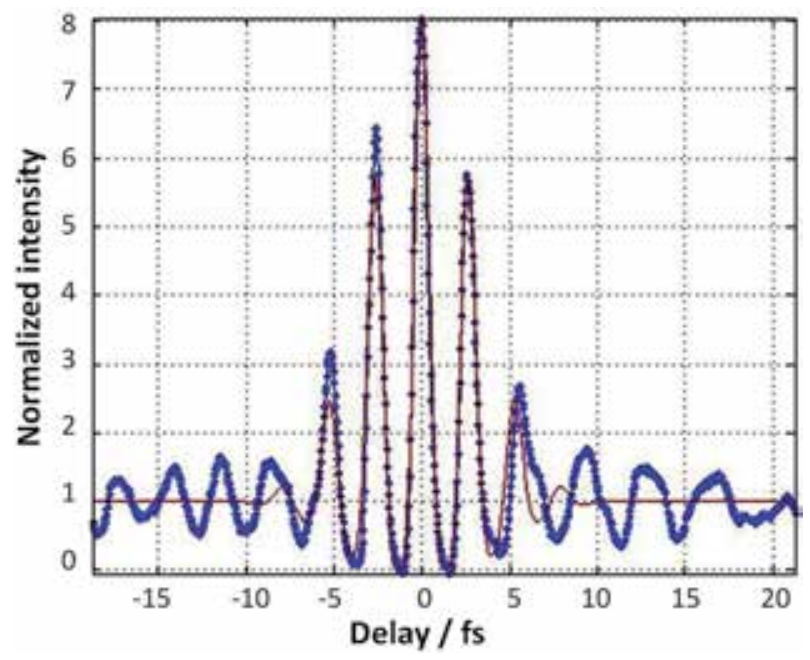

Figure 10.

Autocorrelation trace of laser pulse width measurement.

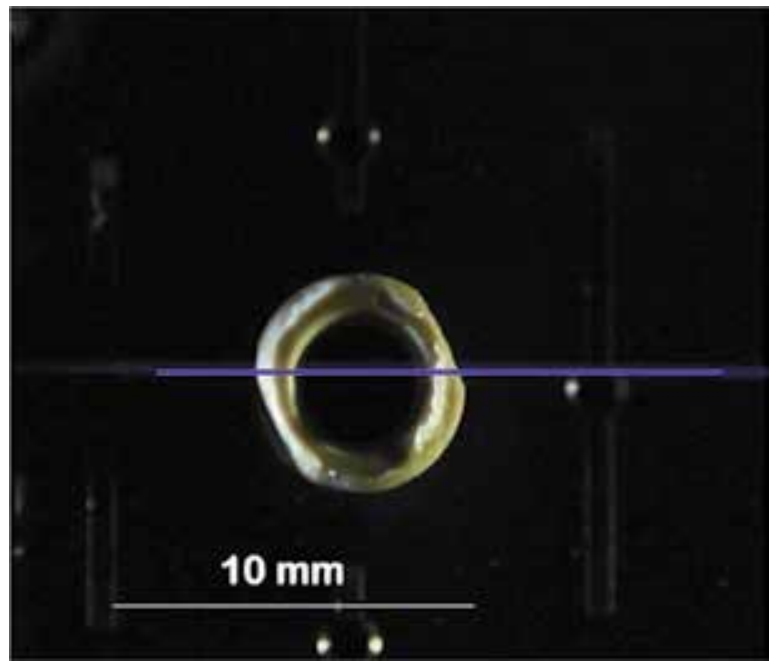

Figure 11.

Strong laser-driven air breakdown plasma.

laser beam spot is just located at the transverse center of the gas tube. At this time the laser power should be too low to make any ablation of the nickel tube. Finally, recover the driving laser power gradually to its experimental value to drill the nickel tube. To reduce the impact of the surrounding air flow on the driving laser beam pointing stability, it is necessary to put the acrylic cover back to get nice tube drilling. It takes at least 30 minutes to finish the drilling process, and the quality can be checked through the pinhole far-field diffraction image, as shown in Figure 12.

This part illustrates the details to achieve the optimum phase matching for the most efficient harmonic conversion output. The zirconium filter of thickness of $0.15-\mu \mathrm{m}$ is used, and its spectral transmittance characteristics are shown in Figure 13. When the HHG chamber reaches the required degree of vacuum, $2 \times 10^{-4} \mathrm{~Pa}$ or even lower, send the driving femtosecond laser and open the neon gas pipeline control valve to generate high-order harmonics. The high-order 
harmonics signal is collected by an X-ray CCD. For the given driving laser settings and nickel tube position, the maximum high-order harmonics yield can be found by finely tuning the backing pressure of the incoming neon gas. By changing the nickel tube position, one can obtain the dependency of high-order harmonics yield on the relative position between the driving laser focus and the target source.

The results are shown in Figure 14, in which the horizontal ordinate of 0 indicates the focus point of the driving laser traveling along the positive direction. The optimum phase matching can be arrived when the nickel tube gas target is placed at about 4-mm after the driving laser focus, indicated by the maximum highorder harmonics yield there. The harmonics yield is showing significant asymmetry and is extremely low for the target position before the driving laser focus, which is different from the result of reference [9]. The beam profiles of the high-order harmonics detected by CCD and the driving field at the gas target are shown in Figures 15 and 16, respectively. It shows that, under the condition of optimum harmonics phase matching with gas target positioned about 4-mm after the driving laser focus, the harmonics generated have similar intensity spatial distribution with the driving laser field, providing an experimental evidence for the commonly used

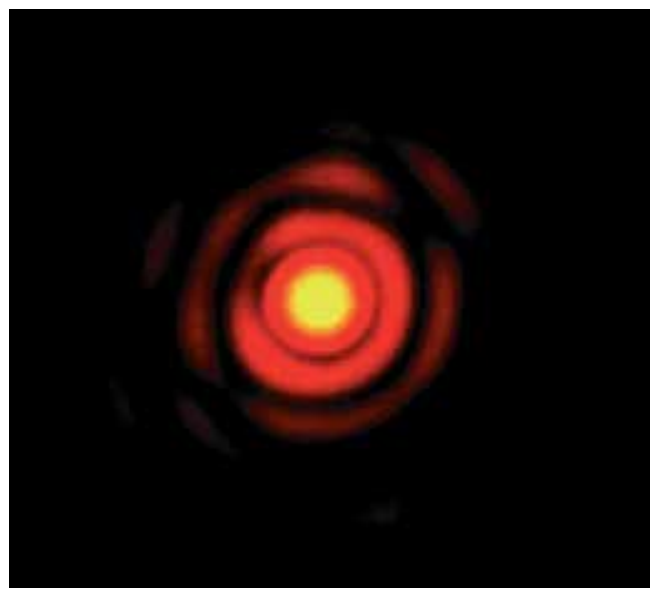

Figure 12.

Far-field pinhole diffraction image of the laser-drilled nickel tube.

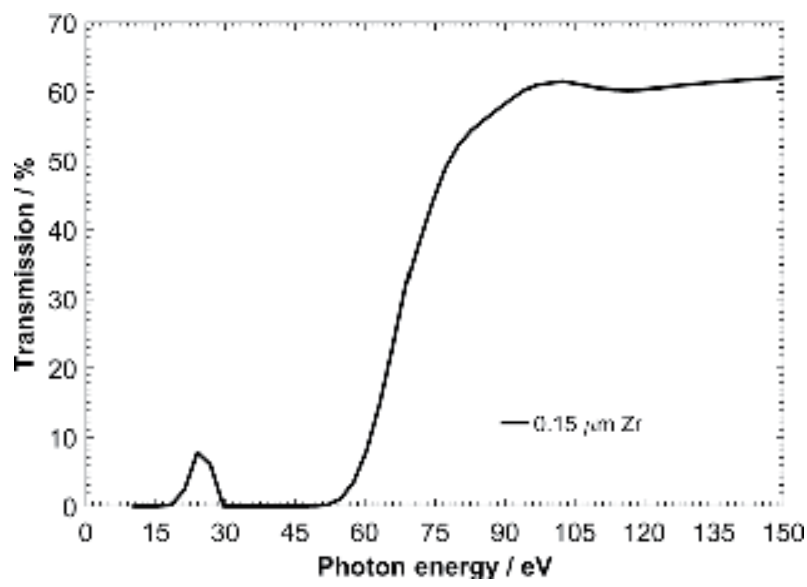

Figure 13.

Transmittance characteristics of 0.15- $\mu m$ zirconium filter [32]. 
Modern Applications of Electrostatics and Dielectrics

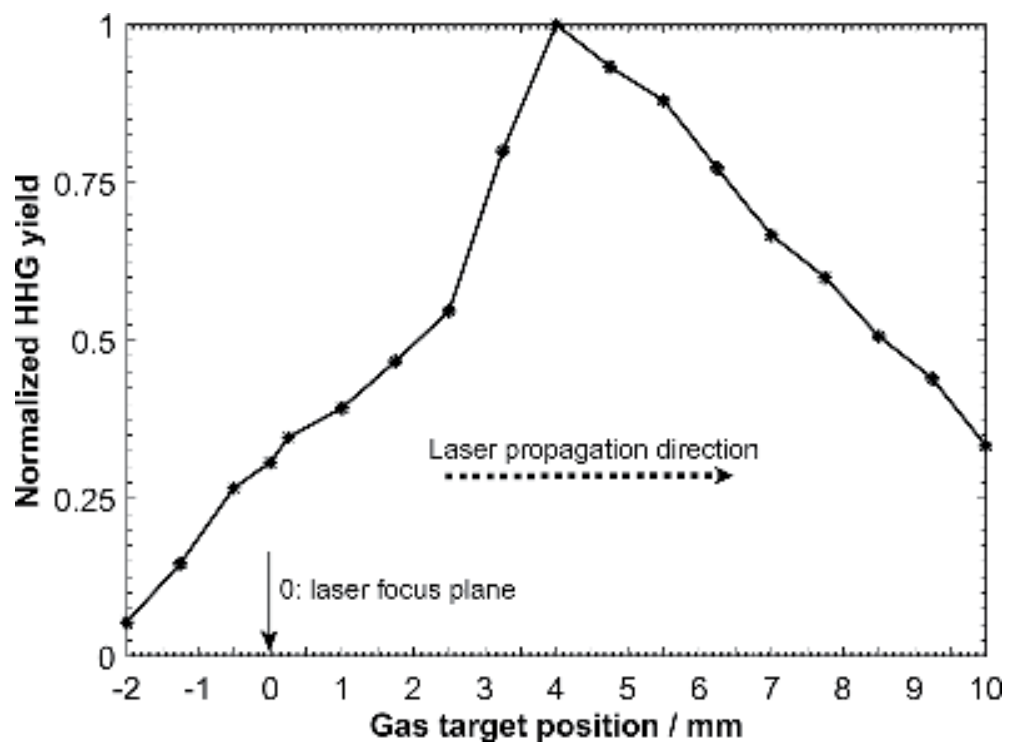

Figure 14.

Dependence of high-order harmonics yield on gas target position.

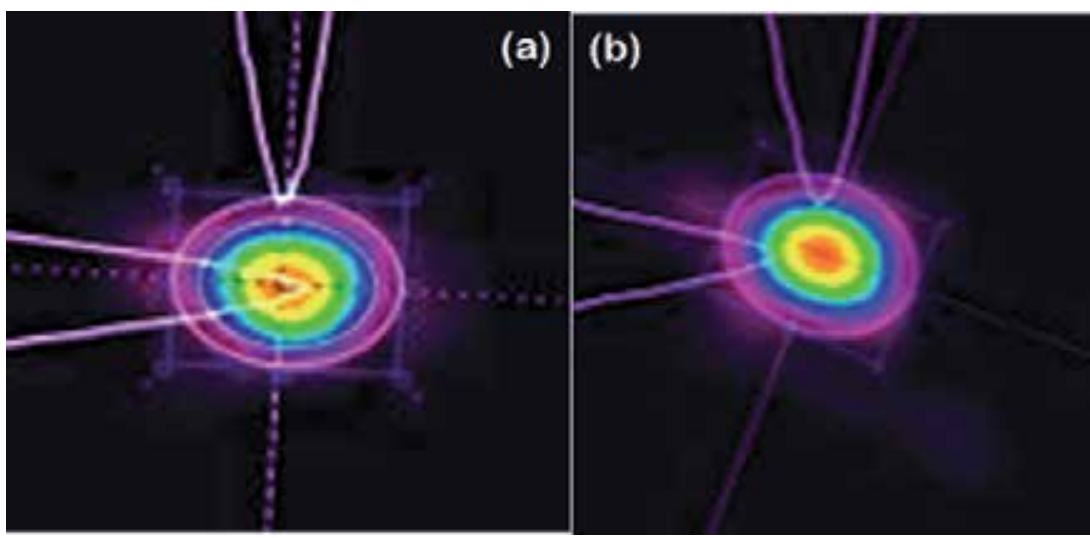

Figure 15.

Driving laser profiles at (a) 4-mm and (b) 2.5-mm after its focus.
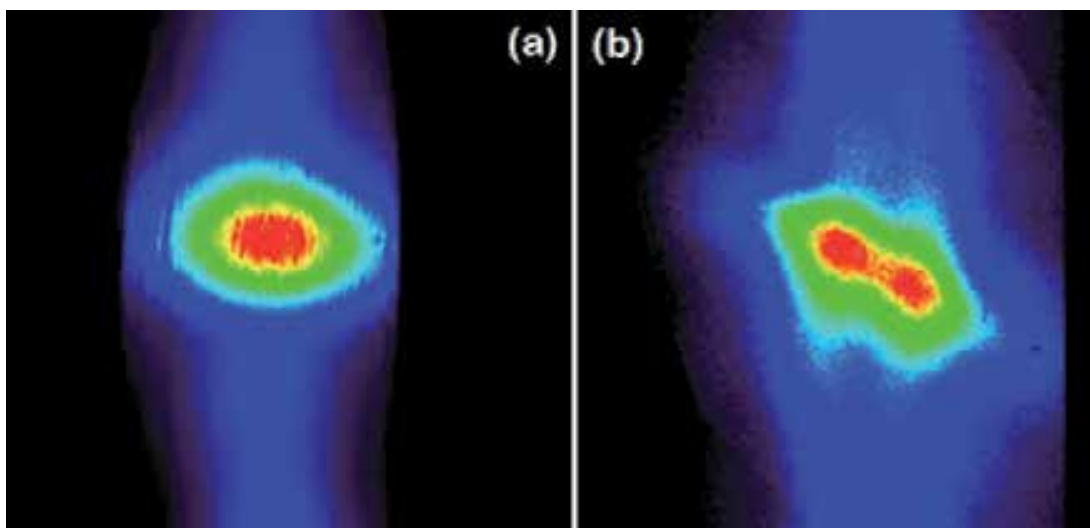

Figure 16.

Far-field profiles of the high-order harmonics with gas target positioned at (a) 4-mm and (b) 2.5-mm after the driving laser focus. 


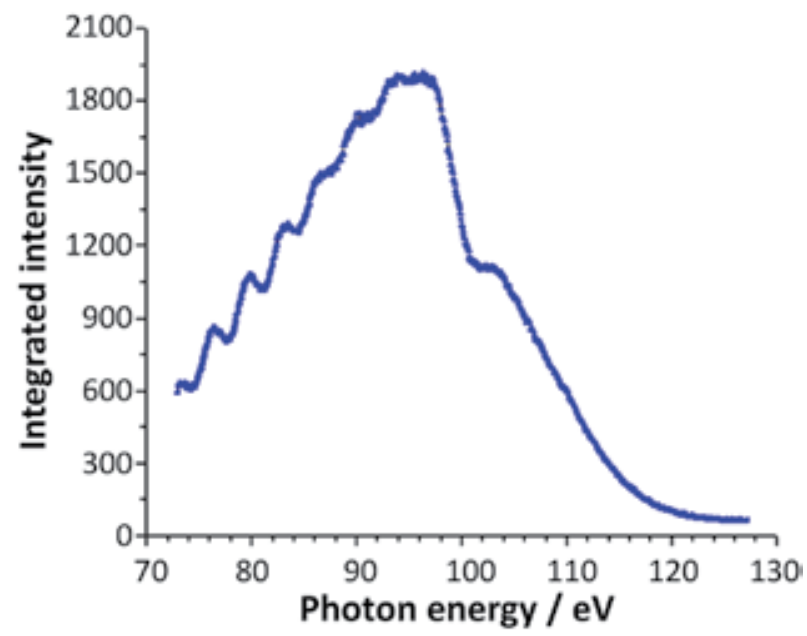

Figure 17.

High-order harmonics spectrum.

assumption that the single isolated attosecond pulse generated by a Gaussian femtosecond laser is also a Gaussian beam. But for the case of worse phase matching as in Figure 15(b), a Gaussian driving laser does not necessarily generate high-order harmonics with similar profile. Using a grazing incidence flat-field spectrometer, the HHG spectrum is analyzed and shown in Figure 17. The spectrum in the cutoff region is continuum, indicating that only one channel gave its contribution for the HHG as in Figure 8(b).

It is worth mentioning that other nickel tubes with different inner diameter sizes were also tried. The findings showed that optimum phase matching of HHG existing after the driving laser focus keeps the same for all these experimental situations, though the gas target position for best harmonic phase matching, the harmonics yield, and the backing pressure of gas target used vary to some extent. So we can safely say that the conclusion is universal for Gaussian-type driving laserbased HHG process.

\section{Conclusions}

The establishment of novel attosecond light source gave rise to an attosecond research upsurge from physics, chemistry, and material science to information processing, among which high-energy single isolated attosecond pulse resulted by interaction between strong optical field and noble gases which has been attracting much attention. Phase matching between the fundamental driving laser field and the high-order harmonics resulted is the key issue to such frequency conversion. In this chapter we scrutinized the intrinsic phase of high-order harmonics resulted from the atom tunneling ionization induced by strong laser field and analyzed qualitatively the salient dependence of polarization gating and amplitude gating on CEP of femtosecond driving laser. The conclusion is that the optimized CEP of driving femtosecond laser for generating single attosecond pulse is $\pi / 2$ and 0 for techniques of polarization gating and amplitude gating, respectively. Meanwhile, an implementation was presented experimentally to exemplify the details for optimum HHG phase matching in the interaction of Gaussian-shaped high-intensity fewcycle femtosecond laser with inert gas target. We studied the dependence of the harmonics phase matching on the relative position between the gas target source 
and driving laser field focus and found that the optimum gas target position for HHG phase matching is always lying behind the focus of the driving field.

\section{Acknowledgements}

The work was supported by the National Natural Science Foundation of China (Grant No. 11675258, 11505289, 61690222).

\section{Author details}

Chao Wang ${ }^{1 *}$, Yifan Kang ${ }^{2}$ and Yonglin Bai $^{1}$

1 Key Laboratory of Ultra-fast Photoelectric Diagnostics Technology, Xi'an Institute of Optics and Precision Mechanics, Chinese Academy of Sciences, Xi'an, People's Republic of China

2 School of Science, Air Force Engineering University, Xi'an, People's Republic of China

*Address all correspondence to: igodwang@163.com

\section{IntechOpen}

(C) 2020 The Author(s). Licensee IntechOpen. This chapter is distributed under the terms of the Creative Commons Attribution License (http://creativecommons.org/licenses/ by/3.0), which permits unrestricted use, distribution, and reproduction in any medium, provided the original work is properly cited. (c) BY 


\section{References}

[1] Wegener M. Extreme Nonlinear Optics. Berlin Heidelberg: SpringerVerlag; 2005. p. 224. DOI: 10.1007/ b137953

[2] Nisoli M, De SS, Svelto O, et al. Compression of high-energy laser pulses below 5 fs. Optics Letters. 1997;22:522-524. DOI: 10.1364/ OL.22.000522

[3] Wegener M. Extreme Nonlinear Optics. 1st ed. Heidelberg: Springer-Verlag Berlin Heidelberg; 2005. p. 223. DOI: $10.1007 /$ b137953

[4] Chang Z. Fundamentals of Attosecond Optics. 1st ed. Florida: CRC Press; 2011. p. 536

[5] Hentschel M, Kienberger R, Ch S, et al. Attosecond metrology. Nature. 2001;414:509-513. DOI: 10.1038/ 35107000

[6] Krausz F, Ivanov M. Attosecond physics. Reviews of Modern Physics. 2009;81:163-234. DOI: 10.1103/ RevModPhys.81.163

[7] Chang Z, Corkum P. Attosecond photon sources: The first decade and beyond [invited]. Journal of the Optical Society of America B: Optical Physics. 2010;27:B9-B17. DOI: 10.1364/ JOSAB.27.0000B9

[8] Calegari F, Ayuso D, Trabattoni A, et al. Ultrafast electron dynamics in phenylalanine initiated by attosecond pulses. Science. 2014;346:336-339. DOI: 10.1126/science. 1254061

[9] Despre V, Marciniak A, Loriot V, et al. Attosecond hole migration in benzene molecules surviving nuclear motion. The Journal of Physical Chemistry Letters. 2015;6:426-431. DOI: 10.1021/ jz502493j
[10] Hassan M. Attomicroscopy: From femtosecond to attosecond electron microscopy. Journal of Physics B: Atomic, Molecular and Optical Physics. 2018;51:1-30. DOI: 10.1088/1361-6455/ aaa183

[11] Goulielmakis E, Schultze M, Hofstetter M, et al. Single-cycle nonlinear optics. Science. 2008;320: 1614-1617. DOI: $10.1126 /$ science. 1157846

[12] Sansone G, Benedetti E, Calegari F, et al. Isolated single-cycle attosecond pulses. Science. 2006;314:443-446. DOI: 10.1126/science. 1132838

[13] Corkum P. Plasma perspective on strong-field multiphoton ionization. Physical Review Letters. 1993;71: 1994-1997. DOI: 10.1103/ PhysRevLett.71.1994

[14] Salieres P, L'Huilier A, Lewenstein M. Coherence control of high-order harmonics. Physical Review Letters. 1995;74:3776-3779. DOI: 10.1103/PhysRevLett.74.3776

[15] Gragossian A, Seletskiy DV, SheikBahae M. Classical trajectories in polorasymmetric laser fields: Synchronous $\mathrm{THz}$ and XUV emission. Scientific Reports. 2016;6:34973/1-34973/8. DOI: 10.1038/srep34973

[16] Kienberger R, Goulielmakis E, Uiberacker $\mathrm{M}$, et al. Atomic transient recorder. Nature. 2004;427:817-821. DOI: $10.1038 /$ nature02277

[17] Witting T, Frank F, Okell WA, et al. Sub-4-fs laser pulse characterization by spatially resolved spectral shearing interferometry and attosecond streaking. Journal of Physics B: Atomic, Molecular and Optical Physics. 2012;45:074014/1-074014/6. DOI: 10.1088/0953-4075/45/7/074014 
[18] Jullien A, Pfeifer T, Abel MJ, et al. Ionization phase-match gating for wavelength-tunable isolated attosecond pulse generation. Applied Physics B. 2008;93:433-442. DOI: 10.1007/ s00340-008-3187-z

[19] Abel MJ, Pfeifer T, Nagel PM, et al. Isolated attosecond pulses from ionization gating of high-harmonic emission. Chemical Physics. 2009;366: 9-14. DOI: 10.1016/j.chemphys. 2009.09.016

[20] Thomann I, Bahabad A, Liu X, et al. Characterizing isolated attosecond pulses from hollow-core waveguides using multi-cycle driving pulses. Optics Express. 2009;17:4611-4633. DOI: 10.1364/OE.17.004611

[21] Ferrari F, Calegari F, Lucchini M, et al. High-energy isolated attosecond pulses generated by above-saturation few-cycle fields. Nature Photonics. 2010;4:875-879. DOI: $10.1038 /$ nphoton.2010.250

[22] Corkum PB, Burnett NH, Ivanov MY. Subfemtosecond pulses. Optics Letters. 1994;19:1870-1872. DOI: 10.1364/OL.19.001870

[23] Sola IJ, Mevel E, Elouga L, et al. Controlling attosecond electron dynamics by phase-stabilized polarization gating. Nature Physics. 2006;2:319-322. DOI: 10.1038/nphys281

[24] Zhao K, Zhang Q, Chini M, et al. Tailoring a 67 attosecond pulse through advantageous phase-mismatch. Optics Letters. 2012;37:3891-3893. DOI: 10.1364/OL.37.003891

[25] Mashiko H, Gilbertson S, Li C, et al. Double optical gating of high-order harmonic generation with carrierenvelope phase stabilized lasers. Physical Review Letters. 2008;100: 103906/1-103906/4. DOI: 10.1103/ PhysRevLett.100.103906
[26] Feng X, Gilbertson S, Mashiko H, et al. Generation of isolated attosecond pulses with 20 to 28 femtosecond lasers. Physical Review Letters. 2009;103: 183901/1-183901/4. DOI: 10.1103/ PhysRevLett.103.183901

[27] Mashiko H, Bell MJ, Beck AR, et al. Tunable frequency-controlled isolated attosecond pulses characterized by either $750 \mathrm{~nm}$ or $400 \mathrm{~nm}$ wavelength streak fields. Optics Express. 2010;18: 25887-25895. DOI: 10.1364/QELS.2011. QMG4

[28] Vincenti H, Quéré F. Attosecond lighthouses: How to use spatiotemporally coupled light fields to generate isolated attosecond pulses. Physical Review Letters. 2012;108: 113904/1-113904/5. DOI: 10.1103/ PhysRevLett.108.113904

[29] Kim KT, Zhang C, Ruchou T, et al. Photonic streaking of attosecond pulse trains. Nature Photonics. 2013;7: 651-656. DOI: 10.1038/NPHOTON. 2013.170

[30] Antoine P, L'Huillier A, Lewenstein $\mathrm{M}$, et al. Theory of highorder harmonic generation by an elliptically polarized laser field. Physical Review A. 1996;53:1725-1745. DOI: 10.1103/PhysRevA.53.1725

[31] An J, Kim DE. Compact in-line autocorrelator using double wedge. Optics Express. 2012;20:3325-3330. DOI: 10.1364/OE.20.003325

[32] Available at: http://henke.lbl.gov/ optical_constants/filter2.html [Accessed: 20 May 2018] 


\title{
Development of a Corona Discharge Ionizer Utilizing High-Voltage AC Power Supply Driven by PWM Inverter for Highly Efficient Electrostatic Elimination
}

\author{
Katsuyuki Takahashi, Koichi Takaki, Isao Hiyoshi, \\ Yosuke Enomoto, Shinichi Yamaguchi and Hidemi Nagata
}

\begin{abstract}
The corona discharge ionizer has been widely used to eliminate electrostatic charges on insulators in a variety of manufacturing industries for the prevention of electrostatic discharge (ESD) problems. High-speed electrostatic elimination is conventionally required for ionizer performance. Because of the high sensitivity of recent electronic devices to ESD damage, an extremely low-offset voltage (ion balance) is required for the performance of electrostatic eliminators. Long-term performance stability is required to maintain the quality of the products, but the short cleaning interval of the unit increases the operating cost. The efficiency is also affected by the waveform of the applied voltage. The optimization of the applied voltage is an important factor in achieving long-term performance stability. In this study, an intermittent pulse voltage AC power supply was developed to achieve a highly efficient electrostatic elimination with long-term stability high-speed electrostatic elimination and an excellent ion balance.
\end{abstract}

Keywords: electrostatic, ionizer, corona discharge, ion balance, high voltage

\section{Introduction}

Elimination of electrostatic charges on insulators for the prevention of electrostatic discharge (ESD) problems in manufacturing industries such as semiconductor and electric device is very important for the enhancement of productivity and reliability [1-5]. The simplest and the most important method to prevent ESD is eliminating charges by keeping conductive materials connected to the ground. In work spaces, wearing anti-ESD wrist straps and conductive clothes and shoes and keeping conductivity of tables and floors high are required for ESD control. In the case of insulators, charges are not removed by connecting them to the ground. Therefore, generally, the charges accumulated on insulators are decayed by decreasing the 
surface resistance by increasing humidity. Although these methods are effective for the prevention of ESD problems, they are restricted by environment, materials, and conditions of objects. When these methods are unavailable, the methods to forcibly eliminate the charges by neutralizing using ions are required.

The corona discharge ionizer has been widely used for elimination of electrostatic charges because of its good operability. Figure 1 shows schematic illustration of elimination of electrostatic charge accumulated on an insulator using an ionizer. The corona discharges are produced at high-voltage electrodes in the ionizer, which enable the production of positive and negative ions such as $\mathrm{H}_{3} \mathrm{O}^{+}, \mathrm{CO}_{3}{ }^{-}, \mathrm{NO}_{3}{ }^{-}$, and their cluster ions $[6-9,10]$. The ions produced by the corona discharges are transported by the electric field or blown air to insulators, and the charges on insulators are neutralized by the ions. The time required for the electrostatic elimination depends on the amount of ions transported to the insulators. For the ionizer performance, not only the short electrostatic elimination time but also the good ion balance (a low-offset voltage), the balance between the amounts of ions of both polarities (Figure 2), is required

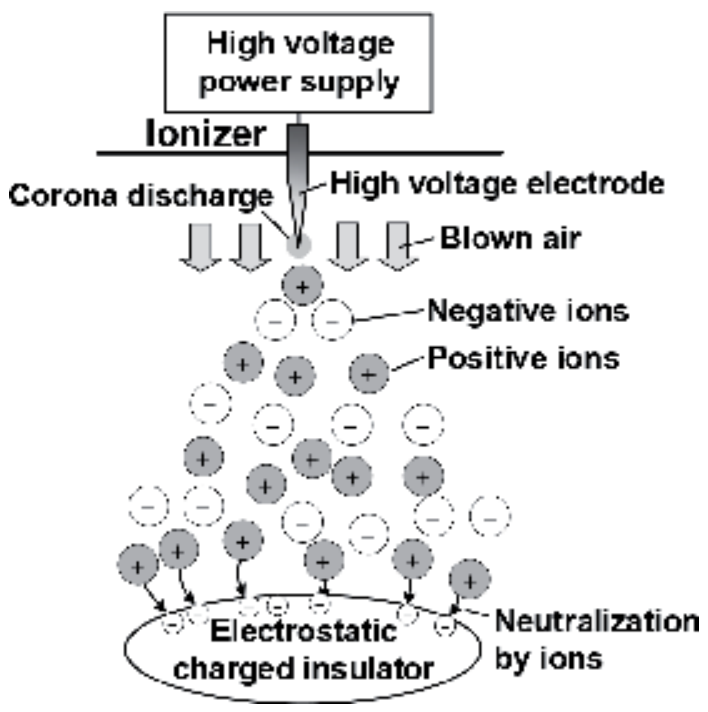

Figure 1.

Schematic illustration of elimination of electrostatic charge accumulated on an insulator using an ionizer.

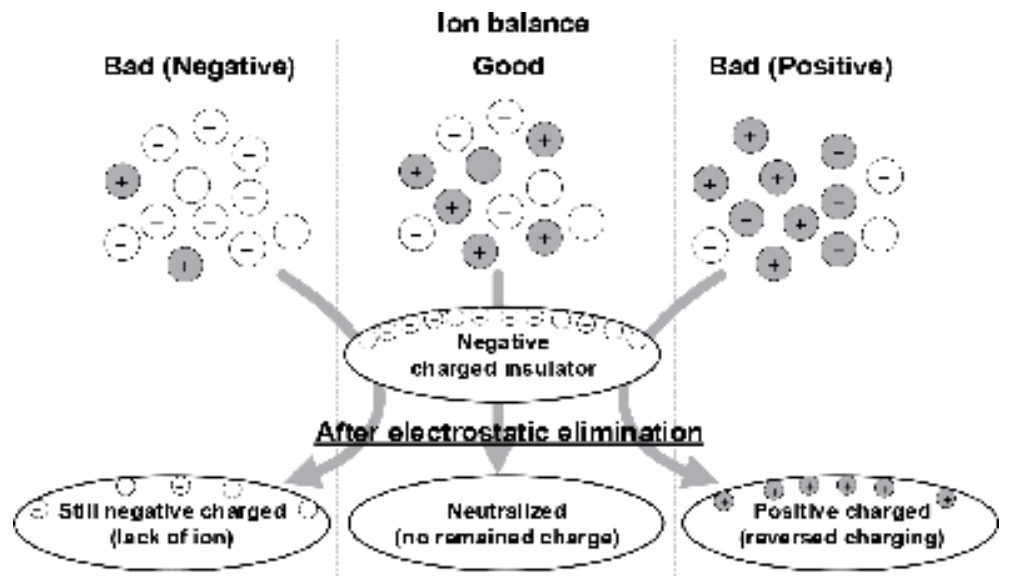

Figure 2.

Relationships between ion balance and electrostatic charges on insulator after electrostatic elimination. 
Development of a Corona Discharge Ionizer Utilizing High-Voltage AC Power Supply Driven... DOI: http://dx.doi.org/10.5772/intechopen.88352

\begin{tabular}{|c|c|c|c|c|c|}
\hline & DC & $\begin{array}{l}\text { Commerclallzed } \\
\text { frequency AC } \\
(50 / 60 \mathrm{~Hz})\end{array}$ & $\begin{array}{c}\text { Rectangular AC } \\
\quad(\leqslant 100 \mathrm{H}<)^{\circ}\end{array}$ & $\begin{array}{c}\text { Intemiltent } \\
\text { blpolar pulse } \\
\text { (several huridied } \\
\mathrm{Hz} \text { ) }\end{array}$ & $\begin{array}{c}\text { High frequency } \\
\text { AC } \\
\text { (steveral ters } \\
\text { kHz) }\end{array}$ \\
\hline $\begin{array}{l}\text { Electrostatic } \\
\text { elmini natlon } \\
\text { without blown alr }\end{array}$ & Excellent & Average & Good & $\begin{array}{l}\text { Below } \\
\text { Avcrage }\end{array}$ & Inferlor \\
\hline $\begin{array}{c}\text { Ion balance } \\
\text { unevenness } \\
\text { In delonzing space }\end{array}$ & Inferior & Average & $\begin{array}{l}\text { Below } \\
\text { Average }\end{array}$ & Good & Excellent \\
\hline $\begin{array}{l}\text { Ion balance } \\
\text { nuctuation }\end{array}$ & Average & Imferior & Inferior & Good & Excellent \\
\hline $\begin{array}{l}\text { Performance } \\
\text { stabifity }\end{array}$ & Inferior & Average & Average & Excellent & Inferior \\
\hline size and welght & Average & Imfentor & $\begin{array}{l}\text { Below } \\
\text { Aver ayge }\end{array}$ & Good & Excellent \\
\hline
\end{tabular}

Table 1.

Characteristics of typical corona discharge ionizers for the various types of voltage supplies.

[11-14]. The bad ion balance causes ESD problem on the latest electronic devices, which have high sensitivity to ESD damage. For example, the ion balance is limited less than 25-100 V in the manufacturing process of electric devices, and only several volts in the process of hard disc heads have a very high sensitivity for ESD [15-18].

The polarity of the ions produced by the corona discharges is the same as the polarity of the applied voltage to the needles $[19,20]$. Table 1 shows the characteristics of the typical corona discharge ionizers for the various types of voltage supplies. The corona discharge ionizers are mainly characterized by the frequency of the applied voltage to the electrodes. There are advantages and disadvantages with each frequency, which require an optimum selection for each situation.

The charge decay time, the time required to eliminate electrostatic charge on a charged object, is significantly affected by the amplitude of applied voltage to the needles, the voltage waveform, and its timing and frequency. Usually, the ions are transported to the charged objects by blown air using a fan or compressed air. When the blown air is limited, the ions must be transported by Coulomb force along the electric field from the needle and by self-diffusion [21]. In this case, DC and lowfrequency and commercialized AC are suitable for electrostatic elimination because the polarity change of the electric field is slow and ions are efficiently transported to the object; on the other hand, the fluctuation width of the ion balance becomes large with low-frequency AC [21]. The fluctuation causes ion balance unevenness in the deionizing space $[20,22,23]$. Although a high-frequency AC requires blown air for electrostatic elimination due to its rapid polarity change, the well-mixed positive and negative ions suppress the fluctuation of the ion balance.

Long-term performance stability is required to maintain the quality of the products, but the short cleaning interval of the unit increases the operating cost. The performance of the electrostatic eliminator declines with the deterioration of the high-voltage electrode because of dust attraction and abrasion from the corona discharge. The amount of ions produced by corona increases with discharge current which depends on amplitude of applied voltage and geometry of electrode [24, 25], which decreases the charge decay time. On the other hand, the efficiency of electrostatic elimination decreases with increasing discharge current that does not contribute to eliminating electrostatic charge, which causes electrode deterioration. The efficiency is significantly affected by the waveform of the applied voltage. DC-type ionizers have two polarity electrodes. Since the abrasion of the electrode depends 
on the polarity, the balance of the positive and negative ions collapses. In the high-frequency AC ionizers, the ions are easily lost by the ion-ion recombination reaction, and the efficiency for ion transport to the charged object is very low. The optimization of the applied voltage is an important factor in achieving long-term performance stability.

In this study, an intermittent pulse voltage AC power supply with a small and lightweight transformer was developed for highly efficient electrostatic elimination. A corona ionizer driven by a power supply offers long-term performance stability in low-offset voltage. The high stability enables the installation of an electrostatic eliminator in the manufacturing processes of high electrostatic-sensitive devices required for extremely low-offset voltage. In this chapter, the specifications of the power supply and the electrostatic eliminator are outlined with quantitative data.

\section{Bipolar pulse voltage power supply driven by PWM converter}

Figure 3 shows a schematic diagram of the bipolar pulse voltage power supply driven by a full-bridge PWM converter [26]. The primary current of the transformer is controlled by a full-bridge converter circuit consisting of four MOSFETs with a DC voltage supply $\left(V_{\mathrm{DD}}\right)$. The value of $V_{\mathrm{DD}}$ depends on the turn ratio of the transformer and is $24 \mathrm{~V}$ for this example. The MOSFETs are driven by PWM input signals $\left(\mathrm{Q}_{1}-\mathrm{Q}_{4}\right)$ generated by a microcomputer through the FET driver circuits. The ON signal and PWM signal are outputted to $Q_{1}$ and $Q_{4}$, respectively, in a positive phase; the $O N$ signal and PWM signal are outputted to $Q_{3}$ and $Q_{2}$, respectively, in a negative phase. Figure 4a shows the relationship between the output voltage and the control signals. The amplitude of the pulse voltage is adjusted by the duty ratio of the PWM signals at the rising edge of the pulse. The PWM signals at the falling edge of the pulse suppress the ringing induced by the resonance, which contributes to generating a pulse shape close to an ideal condition. Figure 5 shows the typical waveform of the output voltage controlled by the PWM signals. The amplitude, interval, and generation timing of the pulses can be arbitrarily set according to the PWM signals as shown in Figure 4b, which realizes the generation of not only intermitted pulse voltage but also AC sinewave voltage.

The frequency and pulse width of the output voltage are determined by the characteristics of the transformer such as the material and the cross section of the core and the number of turns of the coil. Since the characteristics of the

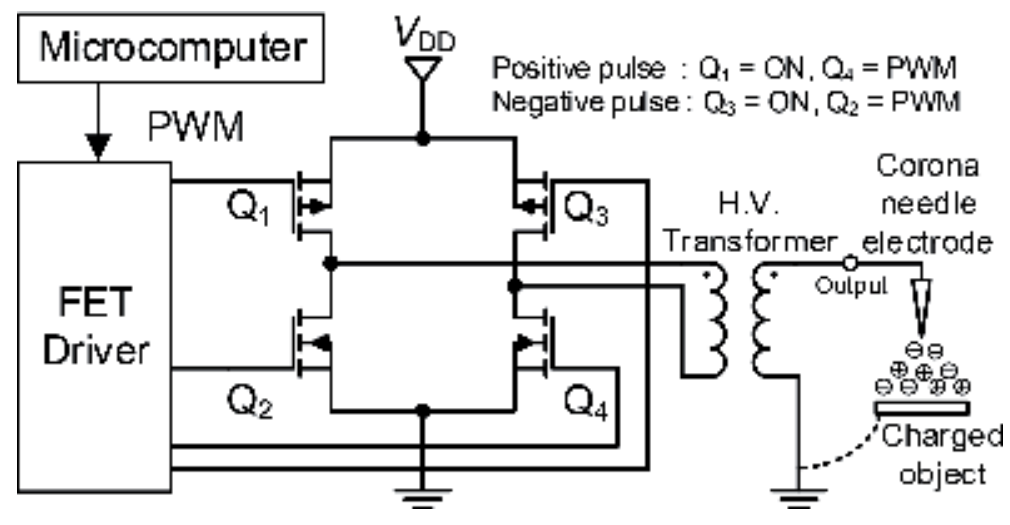

Figure 3.

Schematic diagram of the bipolar pulse voltage power supply driven by a full-bridge PWM converter [26]. 
Development of a Corona Discharge Ionizer Utilizing High-Voltage AC Power Supply Driven... DOI: http://dx.doi.org/10.5772/intechopen.88352

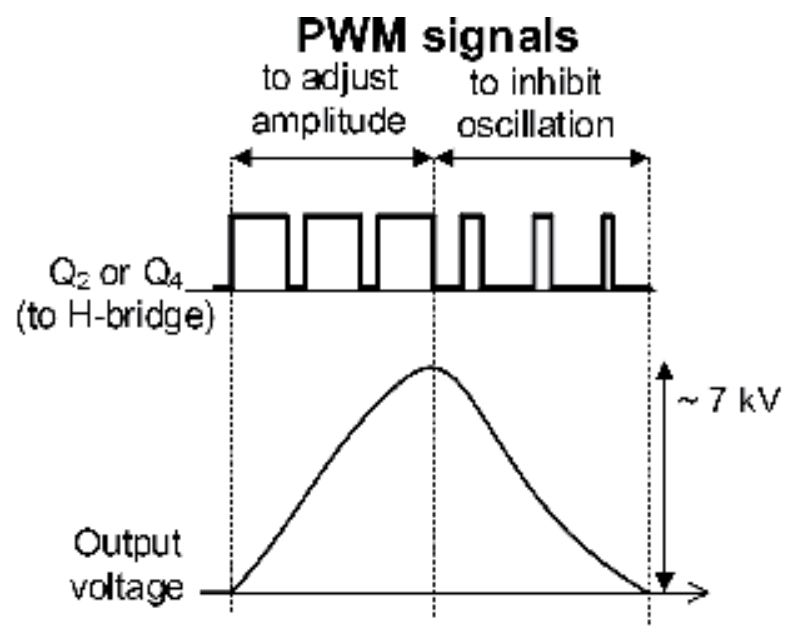

(a)

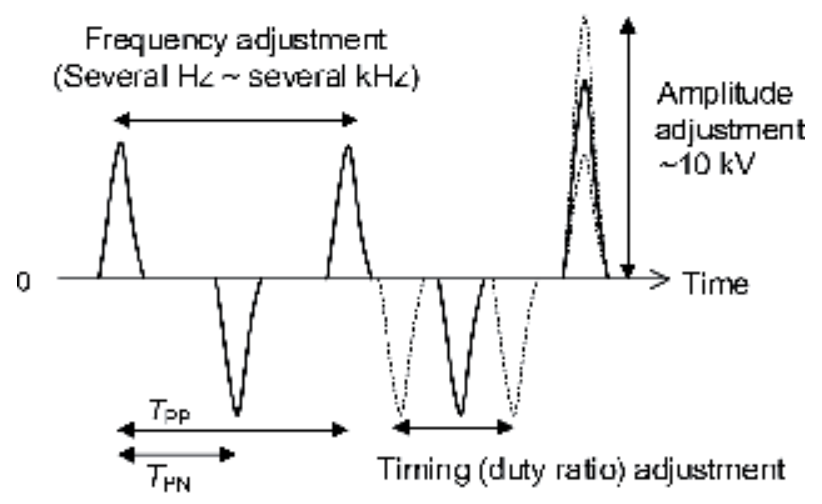

Pulse duty ratio $=\left(T_{1,1}-T_{1 \mathrm{~N}}\right) ! T_{11}, \times 100[\%]$

$T_{\mathrm{p}}$ : Interval between positive to positive pulses

$T_{\Gamma \mathrm{N}}$ : Interval between positive to negative pulses

(b)

Figure 4.

Schematic diagram of PWM control [26]. (a) Relationship between the output voltage and the control signals, (b) output voltage.

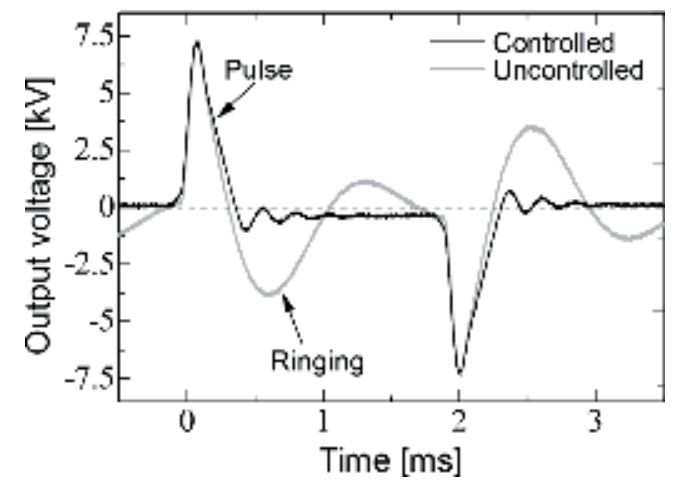

Figure 5.

Typical waveform of output voltage [26]. 


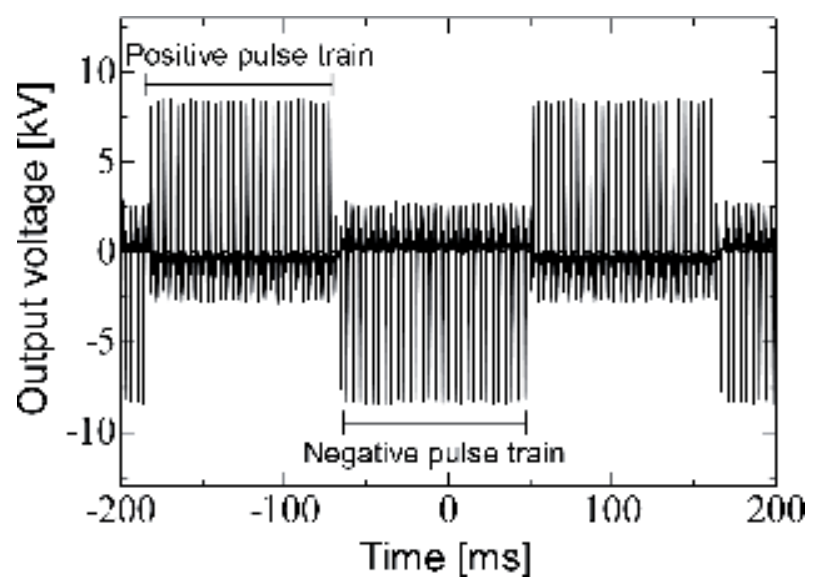

Figure 6.

Typical waveform of continuously generated pulse train [26].

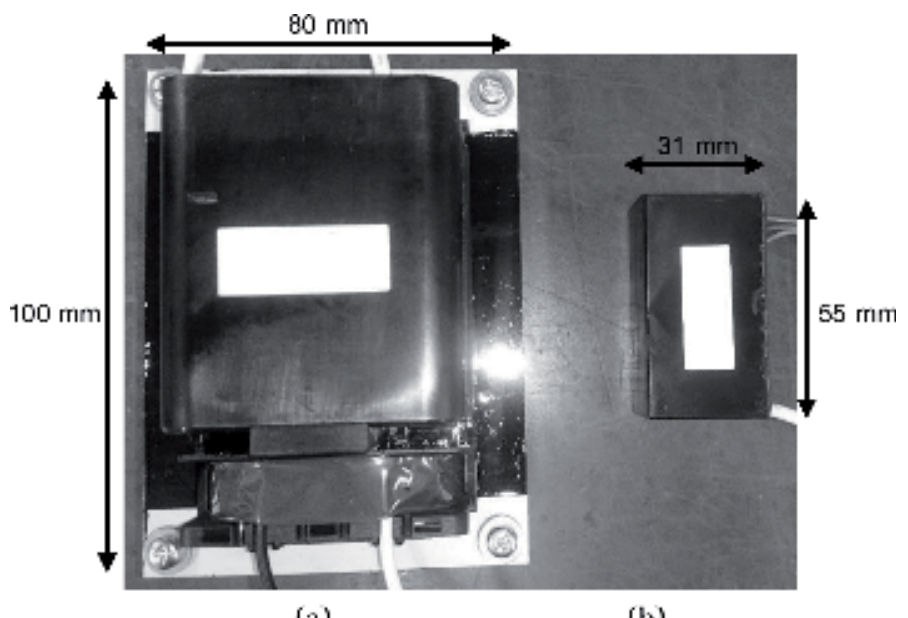

(a)

(b)

Figure 7.

Photograph of two transformers [27]. (a) Commercial frequency, (b) bipolar intermittent pulse.

electrostatic eliminator depend on the frequency as mentioned above, the various types of transformers are prepared for various applications to generate the optimized frequency. Generally, the magnetic flux inside the core required to magnetically couple the primary and secondary windings increases with decreasing the frequency, which increases the size and weight of the transformer. To generate the low-frequency voltage with a small and lightweight transformer, the same polarity pulses are continuously generated in a phase as a pulse train by the PWM converter, and the polarity is switched with a period, as shown in Figure 6. The apparent frequency decreases with increasing number of pulses in the period. The unipolar pulse train can continuously generate unipolar ions in a phase and transport them to the charged object by the electric field and self-diffusion, which contributes to improving the electrostatic elimination speed without blown air, as described later.

The bipolar pulse voltage power supply driven by a full-bridge PWM converter provides a certain degree of freedom in generating the pulse voltages. Figure 7 shows a photograph of a transformer designed for commercial frequency (50 or $60 \mathrm{~Hz}$ ) and a transformer designed for the bipolar intermittent pulse with a frequency of $250 \mathrm{~Hz}$ [27]. The turn ratios are 70 and 360. The cores are both made 
using a silicon steel sheet. Although the transformer designed for the bipolar intermittent pulse is over 10 times smaller and lighter than the transformer designed for commercial frequency, the performance for electrostatic elimination is almost the same in many situations with the optimum voltage waveforms controlled by PWM control. The optimization of voltage waveforms in various applications realizes a design of small and lightweight systems and a long-term performance stability.

\section{Evaluation method of electrostatic elimination performance of ionizer}

The charge plate monitor (CPM) is usually used for evaluation of electrostatic elimination performance of ionizer. CPM consists of a metal plate with a plate-to-

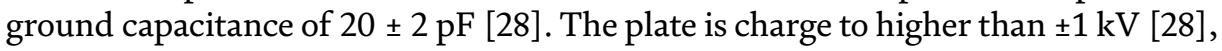
and the voltage is reduced by the ionic current caused by ion flow in the plate. The electrostatic elimination time is defined as time required for decreasing the plate voltage from $\pm 1 \mathrm{kV}$ to $\pm 100 \mathrm{~V}$. The ionic current flow to the plate is determined by the temporal change of the plate voltage according to an equation, $i=\mathrm{d} q / \mathrm{d} t=C \mathrm{~d} v / \mathrm{d} t$. The ion current measuring device such as CPM is required for a high insulation resistance, a low capacitance, and a high-voltage charge because the ionic current into the insulators is on the order of $10^{-9} \mathrm{~A}$ [29]. The frequency response of the CPM is limited by these requirements, which causes an incorrect measurement. For example, the frequency response of the conventional CPMs is lower than $100 \mathrm{~Hz}$. Furthermore, the displacement current induced by the high voltage applied to the corona discharge electrodes interferes with the measurement of the ionic current.

Figure 8 shows a schematic diagram of the ion measuring system developed for a precise evaluation of the ionizer with a high-frequency component [30]. The system consists of the ion-trapping metal plate, capacitors $\mathrm{C}_{\mathrm{I} 1}$ consisted of three series capacitors (WIMA, FKP2-33/1000/10, $33 \mathrm{pF}$ ) and $\mathrm{C}_{\mathrm{I} 2}$ (KEMET, PHE448SB4100JR06, $1 \mathrm{nF}$ ), and a buffer circuit op-amp $\mathrm{A}_{1}$ (Texas instruments, OPA454). The ion-trapping plate voltage $\left(v_{\mathrm{I}}\right)$, usually higher than $\pm 1 \mathrm{kV}$, can be determined by measuring the voltage across $\mathrm{C}_{\mathrm{I} 2}$ using the buffer circuit as a low voltage because the ion-trapping plate voltage $\left(v_{\mathrm{I}}\right)$ is divided by $\mathrm{C}_{\mathrm{I} 1}$ and $\mathrm{C}_{\mathrm{I} 2}$. The buffer circuit does not interfere with measuring $v_{\mathrm{I}}$ because of its very high input impedance $\left(10^{13} \Omega\right)$ and low-bias current $(1.4 \mathrm{pA})$. The ratio between $v_{\mathrm{I}}$ and the output voltage of the buffer circuit $\left(v_{\mathrm{A} 1}\right)$ is determined by the capacitances of $\mathrm{C}_{\mathrm{I} 1}$ and $\mathrm{C}_{\mathrm{I} 2}$. The op-amp

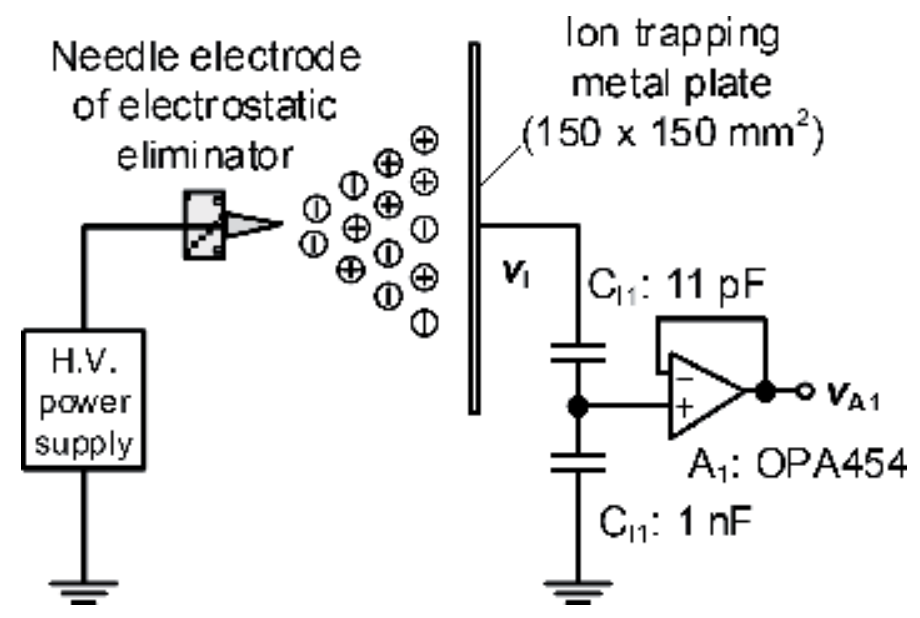

Figure 8.

Schematic diagram of the ion measuring system. 


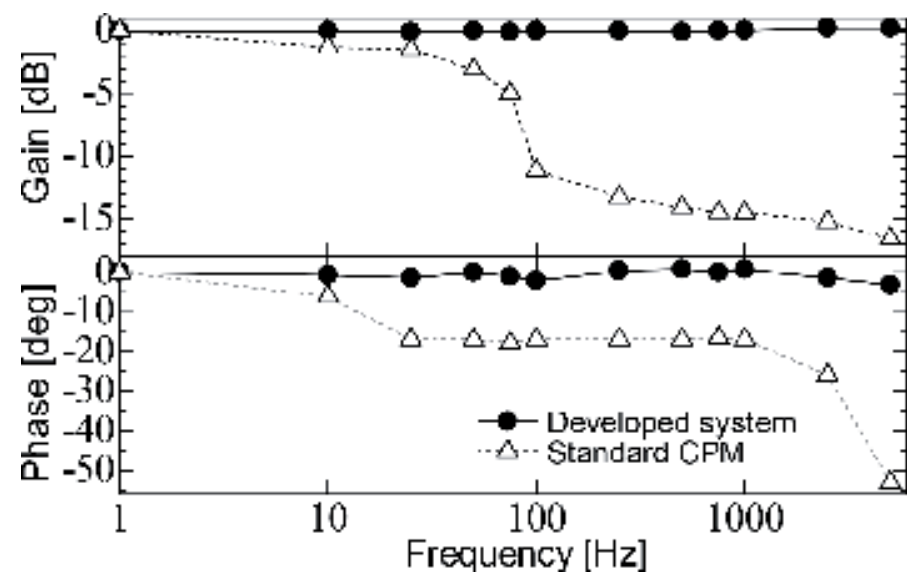

Figure 9.

Bode plots for the developed system and the standard CPM.

allows to apply the input voltage less than $\pm 50 \mathrm{~V}$. Therefore, in the system shown in Figure 6, the maximum voltage that can be applied to the plate is $4.25 \mathrm{kV}$. The total capacitance between the ion-trapping plate and ground is $21.2 \mathrm{pF}$ with the plate area of $150 \times 150 \mathrm{~mm}^{2}$, which is based on a standard established by ESD association [28].

To compare the developed system with a conventional ion evaluation and ion measuring device, a standard CPM (Trek, Model 158) is employed. Figure 9 shows the bode plots for the developed system and the standard CPM [30]. The gain and phase shift have constant values in the case of the developed system with the frequencies lower than $10 \mathrm{kHz}$. Although the gain of the standard CPM drastically decreases with the frequency higher than approximately $60 \mathrm{~Hz}$, that of the developed system has a constant gain without phase shift. The voltage of the plate is maintained with the charge decay time of 83 minutes. The insulation resistance estimated by the decay time according to an equation is $R=t / C$ and is $0.23 \times 10^{9} \Omega$. The time required for decaying $5 \%$ of initial charging voltage is 243 seconds, which is satisfied for the evaluation of the performance of the corona discharge ionizer [28].

Figure 10 shows the typical waveform of the charged voltage of the developed CPM in the case of evaluating the charge decay time of an ionizer. The initial charge voltage to the plate is $\pm 1.1 \mathrm{kV}$ and is decayed exponentially by the ions generated by the ionizer. Generally, the charged decay time is defined as the time required to decay to $\pm 0.1 \mathrm{kV}$ from $\pm 1.0 \mathrm{kV}$. When the plate voltage assumes a steady-state condition after electrostatic elimination, the average value of the plate voltage is defined as the ion balance or the offset voltage.

Figure 11 shows the equivalent circuit model of the electrostatic elimination system using a corona discharge ionizer. The ionizer and the charged object are connected by the deionizing space. The $v_{\mathrm{O}}$ is the output voltage of the high-voltage power supply applied to the electrode of electrostatic eliminator. $C_{\mathrm{A}}$ is the stray capacitance and is on the order of $10^{-12} \mathrm{~F}$. $R_{\text {ion }}$ is resistance expressed by the ion flow and is on the order of $10^{8}-10^{10} \Omega$ [27]. $C_{\text {obj }}$ and $R_{\text {obj }}$ are the capacitance and the leakage resistance of the charged object, respectively. Since the high-voltage supply (ionizer) and the object (CPM) are coupled by $C_{\mathrm{A}}$, the voltage synchronized to $v_{\mathrm{O}}$ is induced on $C_{\text {obj. }}$. In the electrostatic elimination system shown in Figure 8, the voltage is induced on the ion-trapping plate of the CPM and is induced by the electric field emitted from the ionizer in the same manner. Additionally, the ions generated by the corona discharges flow into the plate electrode through $R_{\text {ion }}$. The polarity of the ion flows into the plate is the same as the phase of $v_{\mathrm{O}}$. Therefore, 


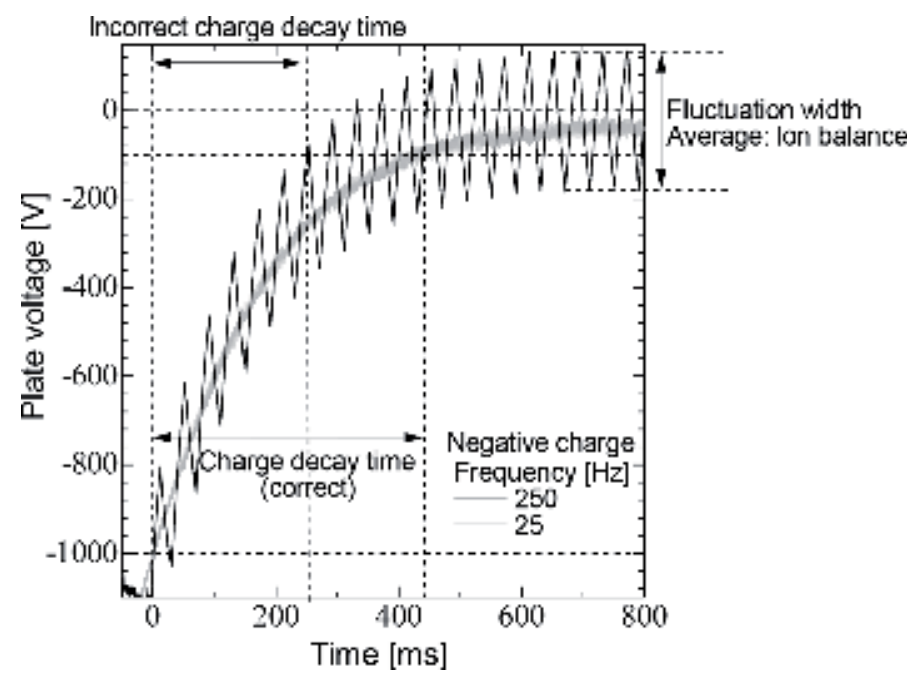

Figure 10.

Typical waveform of the plate voltage in the case of negative charged electrostatic elimination for two frequencies.

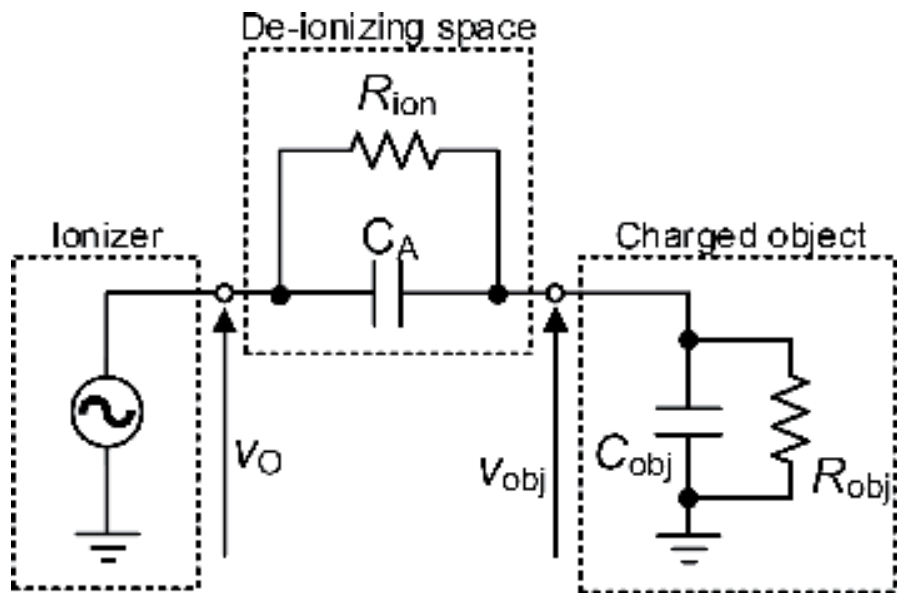

Figure 11.

Equivalent circuit model of the electrostatic elimination system using a corona discharge ionizer.

the plate voltage fluctuates during the steady-state condition after electrostatic elimination. Figure 12 shows the example waveforms of a high voltage applied to an electrode of an electrostatic eliminator, the plate voltage, an induced voltage by high voltage, and a voltage generated by an ionic flow current to the plate. A rectangular waveform with a frequency of $1 \mathrm{kHz}$ is applied to the electrostatic eliminator. The induced voltage is estimated by the electric field from the electrostatic eliminator using a probe [30]. The voltage due to the ionic current is estimated by subtracting the induced voltage from the plate voltage. The plate voltage is induced by the electric field and the ionic current, which causes a fluctuation of the voltage potential of the object.

This fluctuation is submerged when the CPM's frequency characteristics are not sufficiently high for the frequency of the voltage applied to the electrostatic eliminator, which could lead to unexpected ESD problems. Additionally, the fluctuation could lead to underestimation of the charge decay time because some CPMs define 


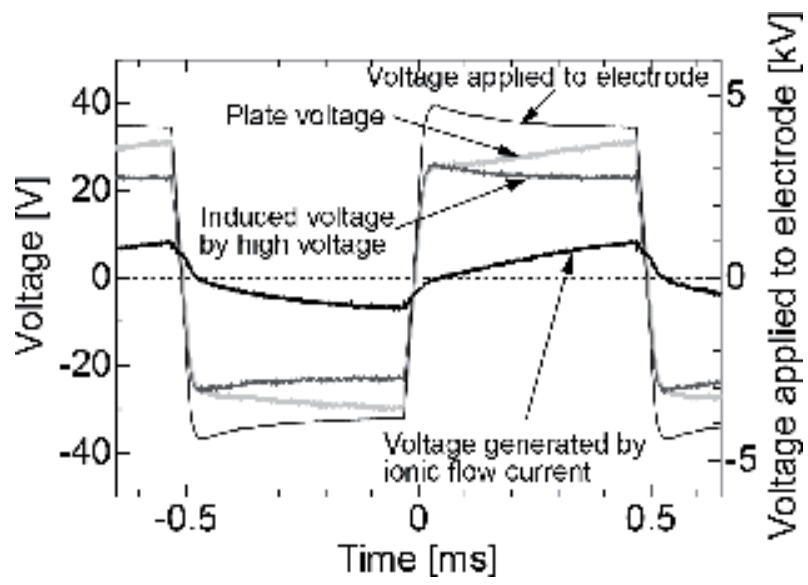

Figure 12.

Example waveforms of a high voltage applied to an electrode of an electrostatic eliminator, the plate voltage, an induced voltage by the high voltage, and a voltage generated by an ionic flow current to the plate.

the charge decay time when the plate voltage reaches to $\pm 0.1 \mathrm{kV}$ at once, although it should be measured with the time averaged voltage as shown in Figure 8. The precise evaluation of the voltage potential of the object is vitally important for high-level ESD control.

\section{Characteristics of electrostatic elimination using bipolar pulse voltage with blown air}

Figure 13 shows the charge decay time as a function of air velocity of the blown air using two intermittent bipolar pulsed voltages, uncontrolled with the ringing and controlled close to an ideal condition, as shown in Figure 5 [26]. A bar-type corona discharge ionizer (Shishido electrostatic, CABX-350L) is used as the ionizer

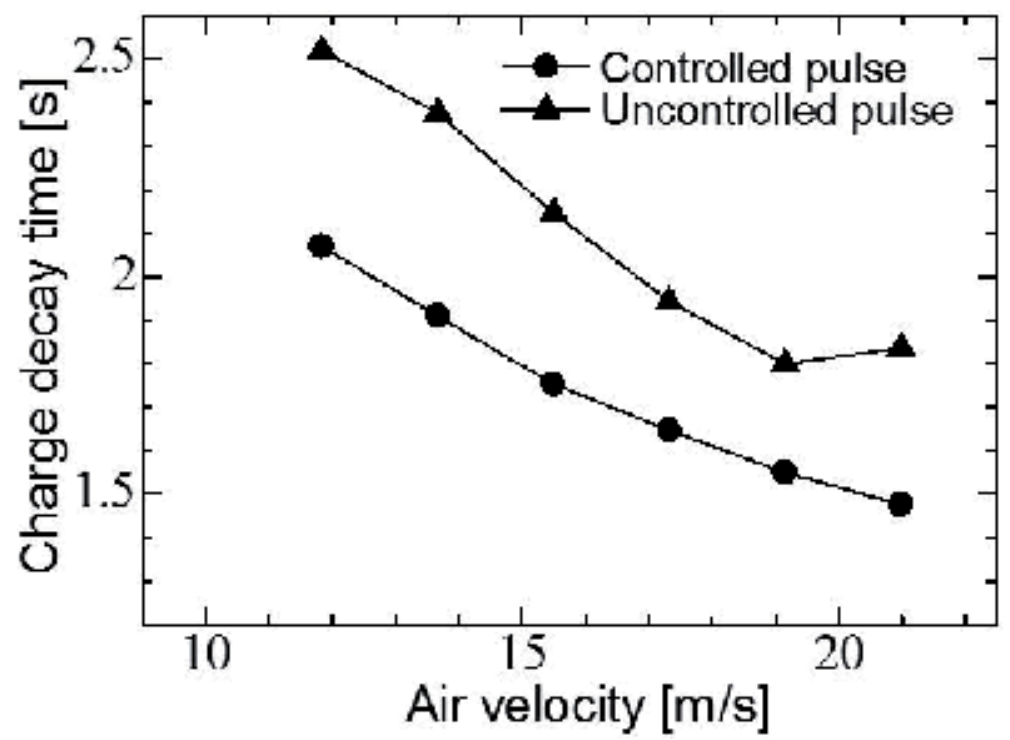

Figure 13.

Charge decay time as a function of air velocity using two intermitted bipolar pulsed voltages, controlled and uncontrolled pulses to inhibit ringing [26]. 


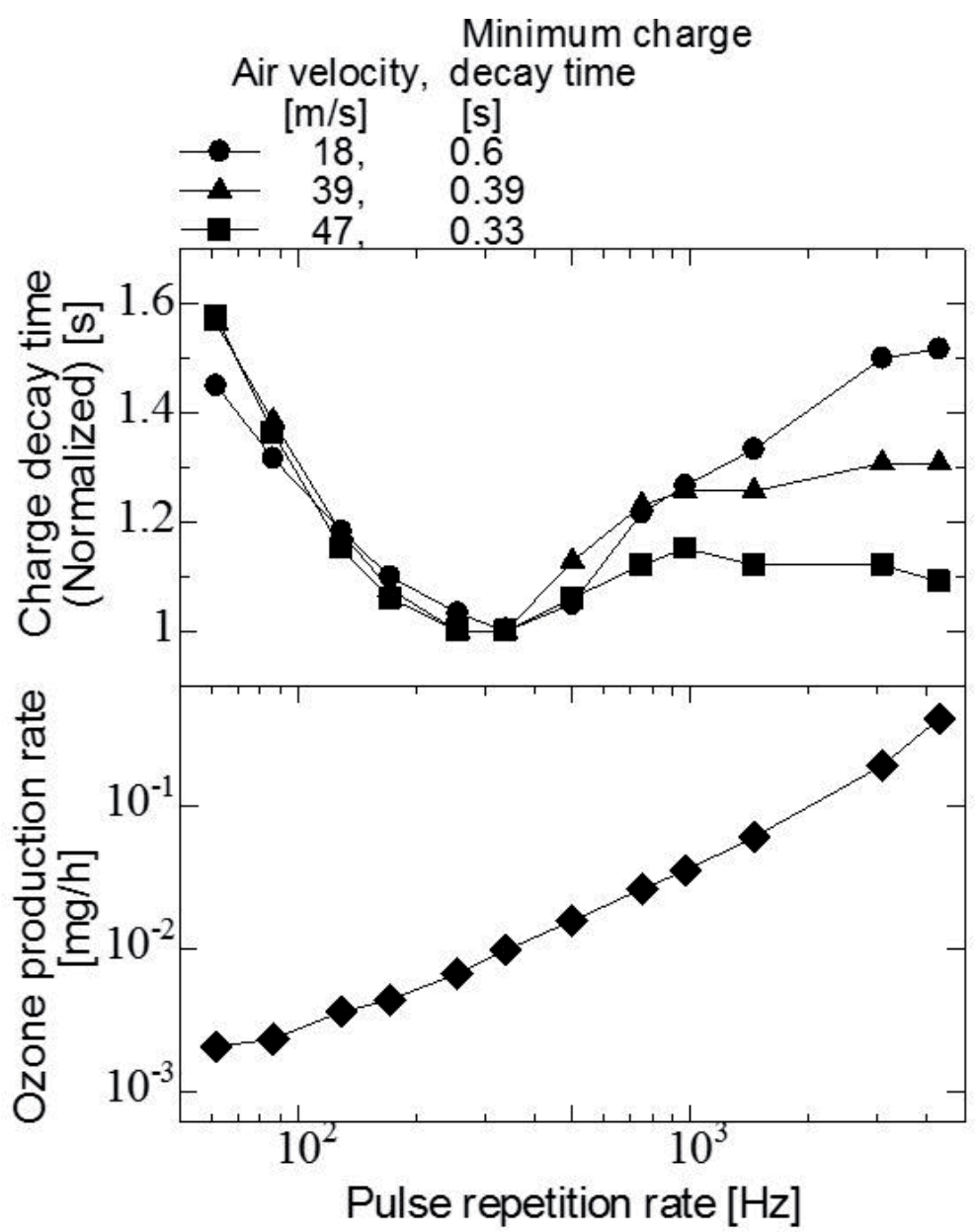

Figure 14 .

Charge decay time and ozone production rate as a function of pulse repetition rate [26].

with the air blown by compressed air. The air velocity is measured at $10 \mathrm{~mm}$ in front of the needles. The amplitude and pulse repetition rate of the pulsed voltage are $7.5 \mathrm{kV}$ and $250 \mathrm{~Hz}$, respectively. The charge decay time in the case of controlled pulsed voltage is much shorter than that of uncontrolled pulsed voltage.

Figure 14 shows the charge decay time and the ozone production rate as a function of the pulse repetition rate. The pulse width is fixed at $0.2 \mathrm{~ms}$. The charge decay time is normalized by the minimum charge decay time for each air velocity. The ozone concentration is determined by an ozone monitor (Ebara, EG-2001). The charge decay time has a minimum at the pulse repetition rate of $250 \mathrm{~Hz}$. The ozone concentration increases with increasing pulse repetition rate. Since ozone is produced by the ionization reactions in the corona discharges, the amount of ions produced by the corona discharges increases with the ozone concentration [31]. The charge decay time depends on the ion flux into the ion-trapping plate and decreases with increasing ion concentration. The number of corona discharges per unit time decreases with decreasing pulse repetition rate, which decreases the ion concentration and increases the charge decay time. On the other hand, with increasing pulse repetition rate, the ions dragged toward the needles by Coulomb force decrease, and the amount of ions lost by the recombination reactions at the vicinity of the needle increases. Therefore, the charge decay time has a minimum value at the pulse repetition rate of $250 \mathrm{~Hz}$. Because the time of flight of the ions decreases with increasing 
the air velocity, the recombination reactions of ions in the deionizing space decrease. Thus, the increase in the charge decay time is suppressed with the higher air velocity, as shown in Figure 14. The uncontrolled pulse waveform with ringing, as shown in Figure 5, has a higher-frequency component than the controlled pulse waveform. This is one of the reasons that the charge decay time in the case of an uncontrolled pulse is higher than that of a controlled pulse as shown in Figure 13.

The corona needle electrodes are degraded with a dust attachment to the electrode, a crystallization of a neutral gas molecule, such as siloxane, at the tip of the electrode, and an erosion of the electrode by an etching effect of corona discharge [32-35]. The performance of the electrostatic eliminator is degraded with needle degradation, which leads to an increase in the charge decay time due to the decrease in ion production and the collapse of the ion balance with unbalanced ion production. The increase in the ions that do not contribute to the electrostatic elimination, such as ions lost by recombination reactions, causes the degradation of the needle electrode, which is strongly affected by the frequency and the waveform of the applied voltage to the electrode. The optimization of frequency and waveform is important for improving the efficiency of electrostatic elimination and maintaining performance during long-term operation.

The ion balance can be controlled by the timing of pulse generation. Figure 15 shows the ion balance as a function of the pulse duty ratio. The pulse duty ratio, a timing of pulse generation, is defined as shown in Figure $5 \mathbf{b}$. The ion balance linearly increases with increasing pulse duty ratio with a controlled pulse, which enables the easy control of ion balance. Generally, the ion balance has been controlled with the amplitude of the voltages in the cases of DC and AC type ionizers. Figure 16 shows the time change of the plate voltage using three different types of high-voltage power supply: DC, a commercialized frequency $(50 \mathrm{~Hz})$, and an intermittent pulse $(250 \mathrm{~Hz})$. The amplitude of the voltage is fixed at $7 \mathrm{kV}$ in every type of high-voltage power supply. The fluctuation of the plate voltage is suppressed using the intermittent pulse type in comparison with the others because the corona discharge and ion production are stable. The ion balance control by the duty ratio of the intermittent pulse enables an easy and rapid control of the ion balance, which also contributes to long-term stability.

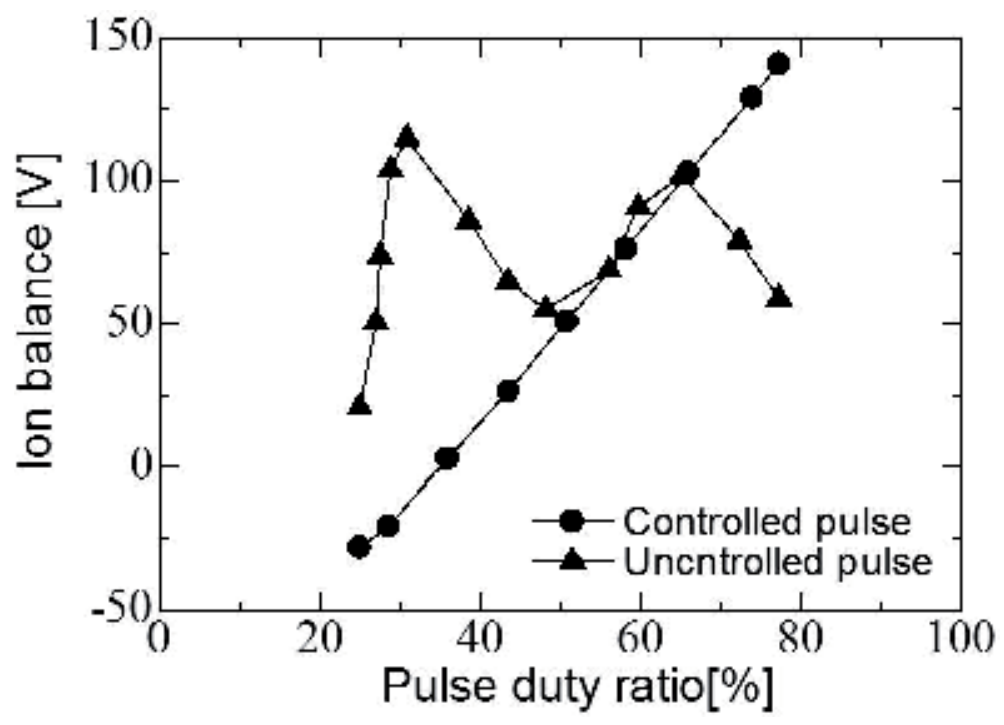

Figure 15.

Ion balance as a function of pulse duty ratio [26]. 


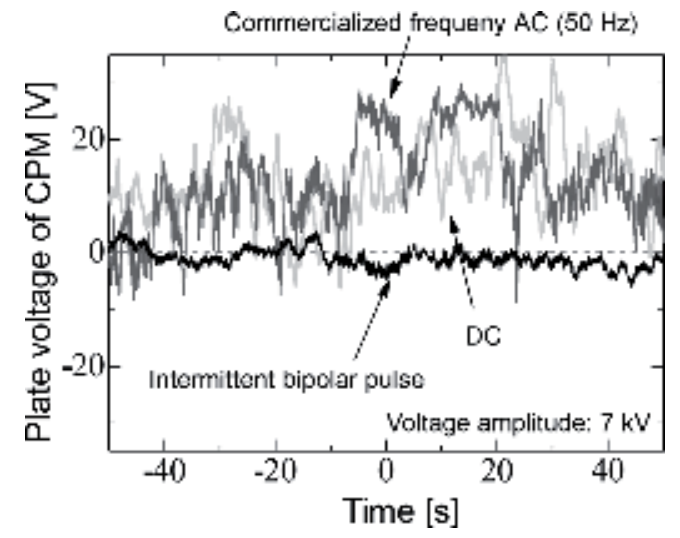

Figure 16.

Time change of the plate voltage using three different types of high-voltage power supply.

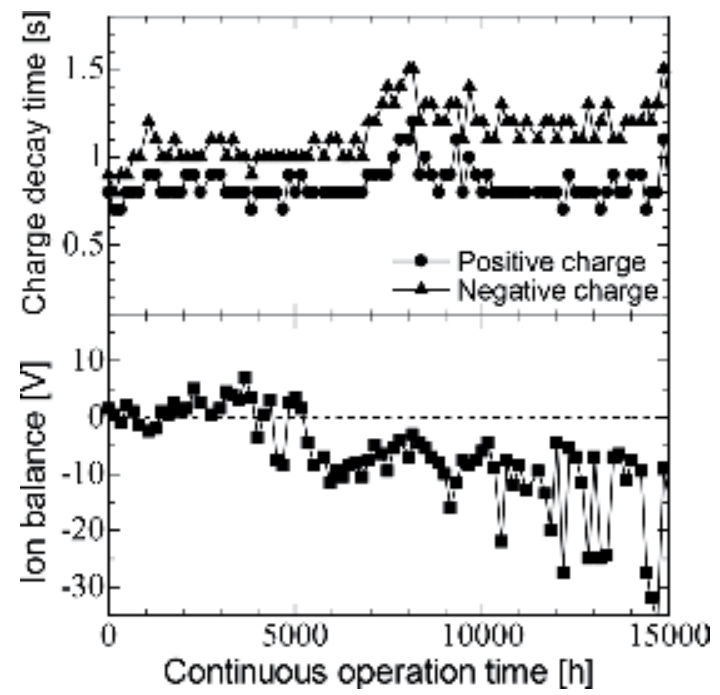

Figure 17.

Time change of the charge decay time and ion balance of a corona discharge electrostatic eliminator driven by the bipolar intermittent pulse voltage.

Figure 17 shows the time change of the charge decay time and the ion balance of a corona discharge electrostatic eliminator driven by the bipolar intermittent pulse voltage. The performance is maintained for 15,000 hours, several times longer than conventional commercial frequency AC and DC types. Recently, a perfectly balanced fan-type ionizer utilizing an intermittent pulse AC voltage power supply has been developed [36]. The short-term fluctuation range of the offset voltage is smaller than $\pm 2 \mathrm{~V}$ without a sensor feedback system, which has maintained 2500 hours of continuous operation. The charge decay times are also maintained. The electric field expanded from this electrostatic eliminator has an insignificant effect on the voltage potential of the object. This ionizer is very promising for electrostatic elimination situations requiring an extremely low-offset voltage (ion balance), such as a magnetic head slider used in hard disc drives where the ion balance is restricted to a maximum of 2-10 V in the manufacturing process $[15,16,17]$. 


\section{Electrostatic elimination without blown air}

Generally, the ions produced by the corona discharges are transported to the electrostatic charged objects by blown air using a fan or a compressed air. The charge decay time decreases with increasing the air velocity as shown in Figure 13 and is mainly dominated by the air velocity [13, 37-41]. However, blown air is limited in many situations, such as the electrostatic elimination of light weight objects and those in a clean room. In these situations, the ions are mainly transported to the objects by the Coulomb force with the electric field between the electrodes and the objects. In the cases of AC and bipolar pulse voltages, the ions are not efficiently accelerated with the rapid polarization change, which causes the decrease of the electrostatic elimination efficiency due to the increase of ion recombination loss. Therefore, a low-frequency voltage waveform is suitable for electrostatic elimination without blown air. However, as mentioned above, in a high-voltage power supply using a transformer, the magnetic flux inside the core required to magnetically couple the primary and secondary windings increases with decreasing frequency, which increases in the size and weight of the transformer. To generate a low-frequency voltage with a small and lightweight transformer, the same polarity pulse train is continuously generated in a phase by the PWM converter, as shown in Figure 6. Figure 18 shows the typical waveforms of the applied voltage and the plate voltage of the CPM using the continuously generated pulses. The plate voltage continuously increases in the phase in which the same polarity pulses are continuously generated. The results show that the ions flowing into the plate and the phase have the same polarity.

When the same polarity current flows to the primary side of the transformer, the magnetic core is magnetically saturated, which decreases the amplitude and pulse width of the output voltage and causes ringing. To prevent magnetic saturation, a counter pulse with a low voltage is generated before the main pulse voltage. Figure 19 shows the typical waveforms of pulse voltage with and without the counter pulses and the bipolar intermittent pulse. The counter pulse prevents magnetic saturation, which inhibits ringing after the main pulse and decreases the amplitude and pulse width of the pulse voltage. Figure 20 shows the charge decay time with and without the counter pulses. Without blown air, the electrostatic charge is efficiently eliminated by the continuously generated pulses. The charge decay time

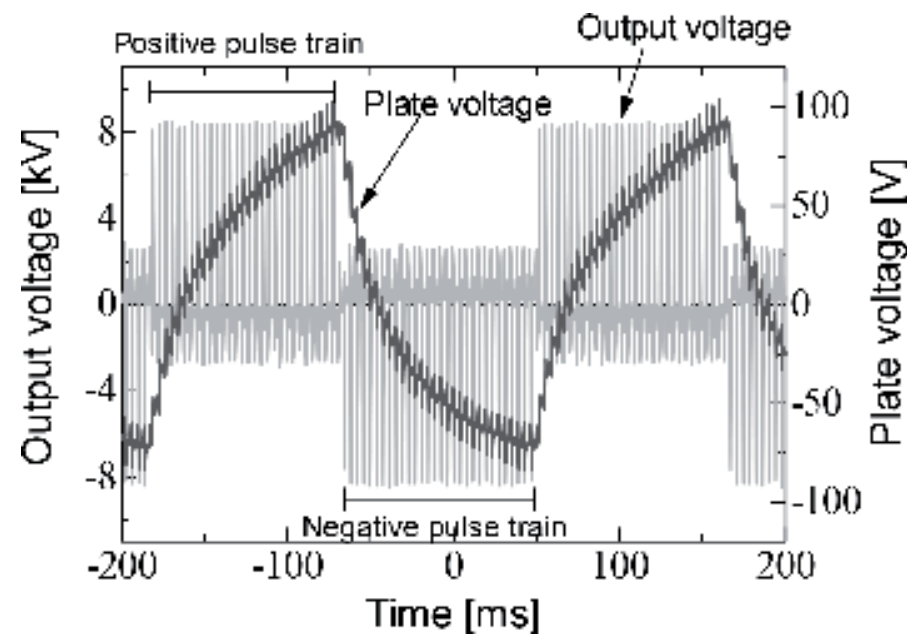

Figure 18.

Typical waveforms of the applied voltage and the plate voltage of CPM. 
Development of a Corona Discharge Ionizer Utilizing High-Voltage AC Power Supply Driven... DOI: http://dx.doi.org/10.5772/intechopen.88352

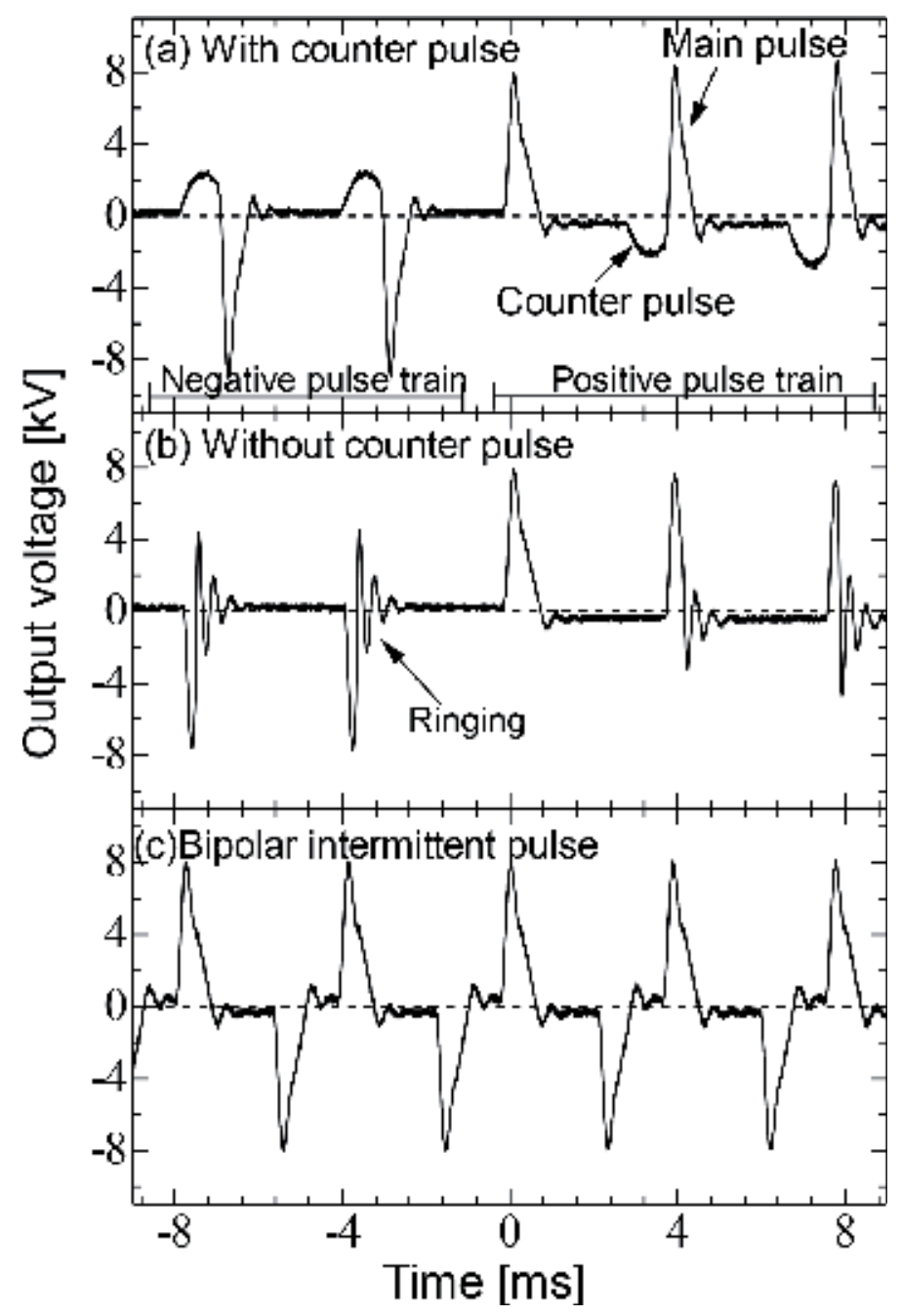

Figure 19.

Typical waveforms of pulse voltage with and without the counter pulses and the bipolar intermittent pulse [26].
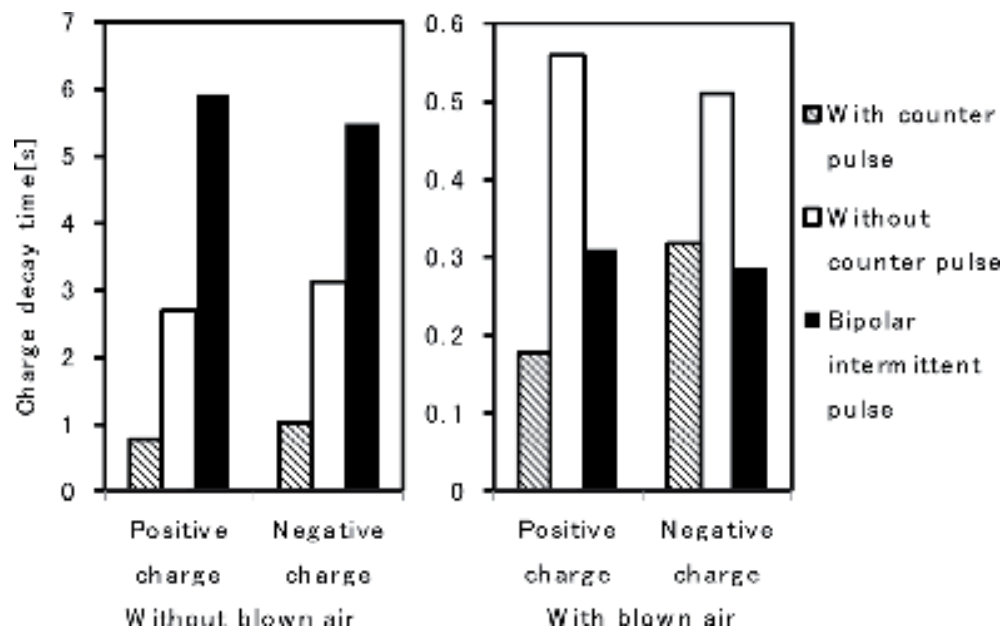

Figure 20.

Charge decay time with and without the counter pulses [26]. 


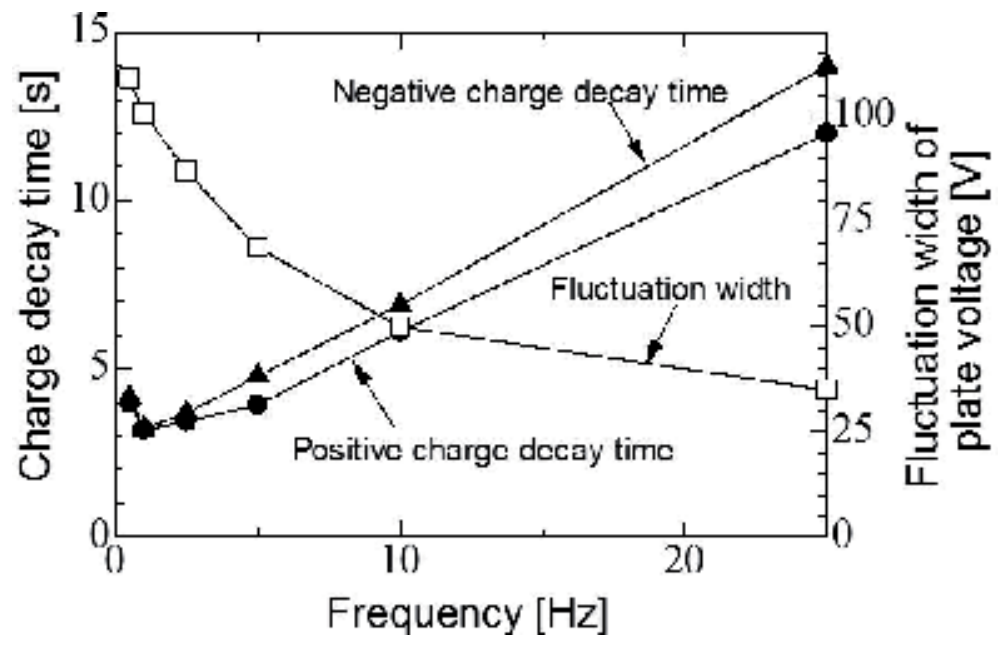

Figure 21.

Charge decay time and the fluctuation width of the plate voltage as a function of the frequency of the voltage phase [26].

with the counter pulses is shorter than that without the counter pulses. In the case of the bipolar intermittent pulse, the charge decay time is over six times longer than that of the continuously generated pulses. The ozone concentration produced by the corona discharges in the case of this method is $0.011 \mathrm{mg}-\mathrm{O}_{3} / \mathrm{h}$ and is lower than that with a conventional commercial frequency AC voltage, at $0.017 \mathrm{mg}-\mathrm{O}_{3} / \mathrm{h}$. The results show that the efficiency of electrostatic elimination is much higher than conventional methods. With blown air Figure 20b, the charge decay time with the counter pulse is shorter than that without the counter pulses. The decrease of the amplitude and pulse width decreases the ion production and the efficiency of ion transportation as shown in Figure 20a.

As shown in Figures 12 and 18, the same polarity ions as the applied voltage continuously flow into the plate at the same polarity phase, and the plate voltage increases according to the polarity. Figure 21 shows the charge decay time and the fluctuation width of the plate voltage as a function of the frequency of the voltage phase. The fluctuation width is measured during the steady-state condition as shown in Figure 10. The charge decay time and the fluctuation width increase with decreasing frequency. Without blown air, the lower frequency is suitable for the electrostatic elimination; however, it causes a large fluctuation of the voltage potential on the objects. When the capacitance and the limitation of the voltage potential of the objects are low, suppressing the fluctuation of the voltage potential is required. The developed method can generate pulse voltages with wide-range frequencies ranging from several $\mathrm{Hz}$ to several $\mathrm{kHz}$ using the same transformer and circuit, which can adjust to various situations.

\section{Conclusion}

An intermittent pulse voltage AC power supply controlled by a PWM inverter with a small and lightweight transformer has been developed for highly efficient electrostatic elimination, and its specifications as an electrostatic eliminator have been highlighted. The PWM control can provide a flexible control of the frequency, pulse width, and amplitude of the voltage waveform applied to the corona discharge electrode. A certain degree of freedom in generating the pulse voltages and the waveforms can be optimized in various applications, which enables the design 
Development of a Corona Discharge Ionizer Utilizing High-Voltage AC Power Supply Driven... DOI: http://dx.doi.org/10.5772/intechopen.88352

of small and lightweight systems. The charge decay time, the time required to eliminate electrostatic charge on a charged object, is evaluated using the charge plate monitor with a high-frequency characteristic for accurate measurements. The efficiency for electrostatic elimination increases with increasing velocity of blow air and has a maximum value with pulse repetition rate of around $250 \mathrm{~Hz}$ in the case of intermittent bipolar pulse voltages. The high-efficiency electrostatic elimination can be realized with the optimization of the waveforms, which contributes to a long-term performance stability. The intermittent pulse voltage AC power supply can contribute to the various processes such as the processes which require very good ion balance.

\section{Conflict of interest}

The authors declare no conflict of interest.

\section{Author details}

Katsuyuki Takahashi, ${ }^{1,2 *}$, Koichi Takaki ${ }^{1,2}$, Isao Hiyoshi ${ }^{3}$, Yosuke Enomoto ${ }^{3}$, Shinichi Yamaguchi ${ }^{1,3}$ and Hidemi Nagata ${ }^{3}$

1 Faculty of Science and Engineering, Iwate University, Morioka, Iwate, Japan

2 Agri-Innovation Center, Iwate University, Morioka Iwate, Japan

3 Shishido Electrostatic, Ltd., Yokohama, Japan

*Address all correspondence to: ktaka@iwate-u.ac.jp

IntechOpen

(C) 2019 The Author(s). Licensee IntechOpen. This chapter is distributed under the terms of the Creative Commons Attribution License (http://creativecommons.org/licenses/ by/3.0), which permits unrestricted use, distribution, and reproduction in any medium, provided the original work is properly cited. (cc) BY 


\section{References}

[1] Greason WD, Castle GSP. The effects of electrostatic discharge on microelectronic devices a review. IEEE Transactions on Industry Applications. 1984;IA-20:247-252. DOI: 10.1109/ TIA.1984.4504404

[2] Lerner A. A new additive for electrostatic discharge control in foams and elastomers. Journal of Cellular Plastics. 1985;21:31. DOI: 10.1177/0021955X8502100103

[3] Vinson JE, Liou JJ. Electrostatic discharge in semiconductor devices: An overview. Proceedings of the IEEE. 1998;86:399-418. DOI: $10.1109 / 5.659493$

[4] Vinson JE, Liou JJ. Electrostatic discharge in semiconductor devices: Protection techniques. Proceedings of the IEEE. 2000;88:1878-1900. DOI: 10.1109/ICMEL.2000.840579

[5] Greason WD, Castle GSP. Review of the effect of electrostatic discharge and protection techniques for electronic systems. IEEE Transactions on Industry Applications. 1987;IA-23:205-216. DOI: 10.1109/TIA.1987.4504895

[6] Nagato K, Matsui Y, Miyata T, Yamauchi T. An analysis of the evolution of negative ions produced by a corona ionizer in air. International Journal of Mass Spectrometry. 2006;248:142-147. DOI: 10.1016/j.ijms.2005.12.001

[7] Nagato K, Nakauchi M. Experimental study of particle formation by ion-ion recombination. The Journal of Chemical Physics. 2014;141:164309. DOI: 10.1063/1.4898376

[8] Inaba H, Ohmi T, Morita M, Nakamura M. Neutralization of wafer charging in nitrogen gas. IEEE Transactions on Semiconductor Manufacturing. 1992;5:359-367. DOI: 10.1109/66.175368
[9] Inaba H, Sakata S, Yoshida T, Okada T, Ohmi T. Antistatic protection in wafer drying process by spin-drying. IEEE Transactions on Semiconductor Manufacturing. 1992;5:234-240. DOI: 10.1109/66.149814

[10] Manninen HE, Franchin A, Schobesberger S, Hirsikko A, Hakala J, Skromulis A, et al. Characterisation of corona-generated ions used in a neutral cluster and air ion spectrometer (NAIS). Atmospheric Measurement Techniques. 2011;4:2767-2776. DOI: 10.5194/ amt-4-2767-2011

[11] Kraz V. Notes on maintaining Sub-1V balance of an ionizer. In: Proceedings of the 2004 Electrical Overstress/Electrostatic Discharge Symposium; 19-23 September 2004; Grapevine, TX, USA; pp. 1-6. DOI: 10.1109/EOSESD.2004.5272608

[12] Levit L, Wallash A. Measurement of the effects of ionizer imbalance and proximity to ground in MR head handling. Journal of Electrostatics. 1999;47:375-382. DOI: 10.1016/ S0304-3886(99) 00040-6

[13] Chang JS. Neutralization theory of static surface charges by an ionizer under wide gas pressure environments. IEEE Transactions on Industry Applications. 2012;37:1641-1645. DOI: 10.1109/28.968172

[14] Takahashi K, Sato M, Ohkubo T, Fujiwara T, Takaki K. 2-D measurement of charged particles diffusing from a double DC corona discharge ionizer. IEEE Transactions on Plasma Science. 2013;41:1863-1868. DOI: 10.1109/ TPS.2013.2252610

[15] Mizoh Y, Nakano T, Tagashira K, Nakamura K, Suzuki T. Soft ESD phenomena in GMR heads in the HDD manufacturing process. Journal of Electrostatics. 2006;64:72-79. DOI: 10.1016/j.elstat.2005.03.090 
[16] Wallash A, Smith D.

Electromagnetic interference (EMI)

damage to giant magnetoresistive

(GMR) recording heads. In:

Proceedings of Electrical Overstress/

Electrostatic Discharge Symposium;

6-8 October 1998; Reno, NV,

USA; pp. 368-374. DOI: 10.1109/

EOSESD.1998.737058

[17] Baril L, Nichols M, Wallash A.

Degradation of GMR and TMR recording heads using very-

short-duration ESD transients.

IEEE Transactions on Magnetics.

2002;38:2283-2285. DOI: 10.1109/

TMAG.2002.802803

[18] Paasi J. Assessment of ESD threats to electronic components. Journal of Electrostatics. 2005;63:589-596. DOI: 10.1016/j.elstat.2005.03.021

[19] Asano K, Fukada Y, Yasukawa T. Measurement of AC ion current from a corona ionizer using a Faraday cage. Journal of Electrostatics. 2008;66:275282. DOI: $10.1016 /$ j.elstat.2008.01.006

[20] Ohsawa A, Nomura N.

Continuously balanced pulse-DC ioniser to minimise the offset voltage. Journal of Electrostatics. 2016;79:16-19. DOI: 10.1016/j.elstat.2015.11.005

[21] Jones JE, Davies M, Goldman A, Goldman B. A simple analytic alternative to Warburgs law. Journal of Physics D: Applied Physics. 1990;23:542-552. DOI: $10.1088 / 0022-3727 / 23 / 5 / 012$

[22] Ohsawa A. Modeling of charge neutralization by ionizer. Journal of Electrostatics. 2005;63:767-773. DOI: 10.1016/j.elstat.2005.03.043

[23] Ohsawa A. Computational comparison of charge neutralisations of conductors and insulators with corona ionisers. Journal of Electrostatics. 2004;62:219-230. DOI: 10.1016/j. elstat.2004.05.009
[24] Dordizadeh P, Adamiak K, Castle GSP. Experimental study of the characteristics of Trichel pulses in the needle-plane negative corona discharge in atmospheric air. Journal of Electrostatics. 2017;88:49-54. DOI: 10.1016/j.elstat.2016.12.013

[25] Frylladitakis ED, Moronis AX, Theodoridis MP. A mathematical model for determining an electrohydrodynamic accelerator's monopolar flow limit during positive corona discharge. IEEE Transactions on Plasma Science. 2017;45:432-440. DOI: 10.1109/TPS.2017.2663778

[26] Takahashi K, Hiyoshi I, Enomoto Y, Nagata H. Development of an electrostatic eliminator utilizing high voltage AC power supply driven by PWM inverter. Journal of International Electrostatics Japan. 2014;38:124-129

[27] Takahashi K, Goto A, Nagata H. Development of an electrostatic eliminator utilizing compact highvoltage AC power supply. Journal of International Electrostatics Japan. 2015;39:238-241

\section{[28] ANSI/ESD STM3.1-2015. ESD} association standard test method for the protection of electrostatic discharge susceptible items-Ionization: ESD Association; 2015

[29] Crowley JM, Leri D, Dahlhoff G, Levit L. Equivalent circuits for air ionizers used in static control. Journal of Electrostatics. 2004;61:71-83. DOI: 10.1016/j.elstat.2003.11.006

[30] Takahashi K, Kaga H, Kubo K, Takaki K, Yamaguchi S, Nagata H. Development of an ion measuring system for AC corona discharge. IEEJ Transactions on Fundamentals and Materials. 2018;138:551-552. DOI: 10.1541/ieejfms.138.551

[31] Chen J, Davidson JH. Electron density and energy distributions in the 
positive DC corona: Interpretation for corona-enhanced chemical reactions. Plasma Chemistry and Plasma Processing. 2002;22(2):199-224. DOI: 10.1023/A:1014851908545

[32] Chen J, Davidson JH. Ozone production in the negative DC corona: The dependence of discharge polarity. Plasma Chemistry and Plasma Processing. 2003;23:501-518. DOI: 10.1023/A:1023235032455

[33] Noll CG, Lawless PA. Comparison of germanium and silicon needles as emitter electrodes for air ionizers. Journal of Electrostatics. 1998;44:221-238. DOI: $10.1016 /$ S0304-3886(98)00015-1

[34] Petrov AA, Amirov RH, Samoylov IS. On the nature of copper cathode erosion in negative corona discharge. IEEE Transactions on Plasma Science. 2009;37:1146-1149. DOI: 10.1109/TPS.2009.2018561

[35] Weissler GL. Positive and negative point-to-plane corona in pure and impure hydrogen, nitrogen and argon. Physical Review. 1943;63:96-107. DOI: 10.1103/PhysRev.63.96

[36] Takahashi K, Goto A, Yamaguchi S, Saito T, Sakamoto K, Nagata H. Development of a perfectly balanced electrostatic eliminator utilizing an intermittent pulse $\mathrm{AC}$ voltage power supply. In: Proceedings of 2015 37th Electrical Overstress/ Electrostatic Discharge Symposium (EOS/ESD), 27 September-2 October 2015; Reno, NV, USA; DOI: 10.1109/ EOSESD.2015.7314753

[37] Ohsawa A. Efficient charge neutralization with an ac corona ionizer. Journal of Electrostatics. 2007;65:598-606. DOI: 10.1016/j.elstat.2007.01.003

[38] Osawa A. 2-D electrohydrodynamic simulations towards zero offset voltage with corona ionisers. Journal of
Electrostatics. 2013;71:116-124. DOI:

10.1016/j.elstat.2012.12.021

[39] Noll C. Charge-carrier extraction from air and nitrogen gas streams that entrain charge from DC corona ionizers. Journal of Electrostatics. 2002;54:271-282. DOI: $10.1016 /$ S0304-3886(01)00170-X

[40] Intra P, Tippayawong N. Design and evaluation of a high concentration high penetration unipolar corona ionizer for electrostatic discharge and aerosol charging. Journal of Electrical Engineering and Technology. 2013;8:1175-1181. DOI: $10.5370 /$ JEET.2013.8.5.1175

[41] Intra P, Tippayawong N. Effect of needle cone angle and air flow rate on electrostatic discharge characteristics of a corona-needle ionizer. Journal of Electrostatics. 2010;68:254-260. DOI: 10.1016/j.elstat.2010.01.008 


\title{
Chapter 4
}

\section{Electrostatic Friction Displays to Enhance Touchscreen Experience}

\author{
Reza Haghighi Osgouei
}

\begin{abstract}
Touchscreens are versatile devices that can display visual content and receive touch input, but they lack the ability to provide programmable tactile feedback. This limitation has been addressed by a few approaches generally called surface haptics technology. This technology modulates the friction between a user's fingertip and a touchscreen surface to create different tactile sensations when the finger explores the touchscreen. This functionality enables the user to see and feel digital content simultaneously, leading to improved usability and user experiences. One major approach in surface haptics relies on the electrostatic force induced between the finger and an insulating surface on the touchscreen by supplying high AC voltage. The use of AC also induces a vibrational sensation called electrovibration to the user. Electrostatic friction displays require only electrical components and provide uniform friction over the screen. This tactile feedback technology not only allows easy and lightweight integration into touchscreen devices but also provides dynamic, rich, and satisfactory user interfaces. In this chapter, we review the fundamental operation of the electrovibration technology as well as applications have been built upon.
\end{abstract}

Keywords: electrostatic display, variable friction display, electrovibration technology, surface haptics, tactile rendering, texture rendering

\section{Introduction}

Among the five senses, touch is the most fundamental one we are equipped from the moment we enter this world. Even newborn babies know how to utilize their sense of touch to interact with their surrounding environment. Many of the typical tasks around us require touch which without it even a very basic task would be challenging to accomplish. Just imagine how difficult it can be to grab any object if you cannot feel its shape and weight or determine the amount of force you need to apply to hold it. Touch is very important to human being, and we rely on our touch sense more than we think we do [1].

Modern technologies in this digital era added new interactive agents around us which require our touch input. Touchscreen consumer electronics such as smartphones and tablet devices are among them. They are a versatile device that displays visual content and takes touch input simultaneously. More specifically, smartphones are an inevitable part of our daily life. Users spend a significant amount of time interacting with the digital contents on their mobile phones. So, equipping such devices with functionality to provide some sort of touch feedback was inevitable and seemed to be a natural course of technological development. 
However, despite technological advances, these devices lack the ability to provide programmed tactile feedback, which can be essential for more natural and intuitive interaction. At best, they provide some simple monotonic vibration patterns in response to the user's touch input. This is neither appealing nor satisfactory given the expectations users have from such modern devices [1].

With the introduction of variable friction displays, this limitation has been addressed by technologies collectively called surface haptics. These technologies modulate friction between a user's fingertip and a touchscreen surface in order to create a variety of tactile sensations when the finger explores on the touchscreen. This functionality allows the user to see and feel the digital content simultaneously with richer haptic information, leading to improved user experience and/ or usability. There exist two major approaches in surface haptics: electrovibration and ultrasonic vibration. Whereas the former increases the surface friction by modulating attractive electrostatic force, the latter decreases the friction by vibrating the surface at an ultrasonic frequency and creating an air gap. Such electrovibration displays have the advantages that they require only electrical components and that the friction can be controlled uniformly on the screen, which are particularly attractive for mobile devices with a provision of adequate amplifiers [2].

The rest of the chapter is organized as following. In the next section, a brief overview of the fundamental operation of the electrovibration technology is given. Next, the literature has been reviewed for the studies, and applications have been built upon. In the final section, conclusions and future remarks are provided.

\section{Electrovibration technology}

The earliest known observation of electrical attraction between the human skin and a charged surface was made by Gray in 1875 [3, 4]. Forgotten for a while, a similar phenomenon was rediscovered later and called electroadhesion by Johnsen and Rahbek in 1923 [4, 5]. In 1953, Mallinckrodt et al. again reported a rubber-like sensation when a coated metallic surface connected to a $110-\mathrm{V}$ power line was touched by a grounded finger $[4,6]$. This phenomenon is called electrovibration by Grimnes in 1983, explaining its principle of operation based on Coulomb's electrostatic force [7]. Electrovibration is due to the electrostatic attraction force between two conductive plates separated by a dielectric. When the finger scans an insulated electrode, a condenser is formed between the electrode and the conductive substance under the skin [7] (Figure 1). Exciting the electrode using a periodic voltage

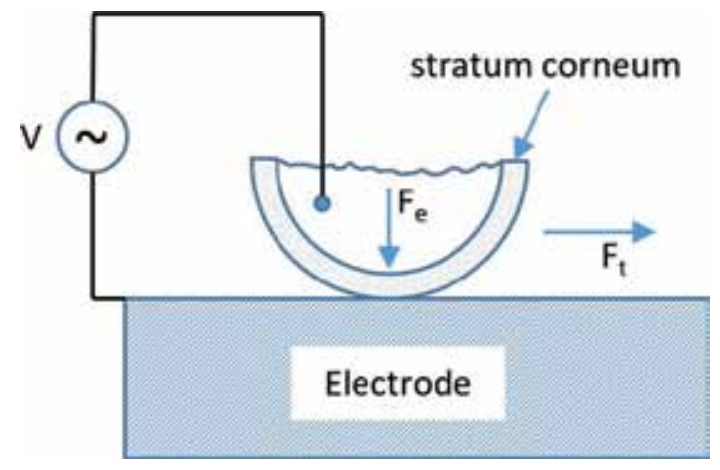

Figure 1.

Interaction between the finger, the isolating part of the skin (stratum corneum), and the conductive plate. 
induces electrostatic attraction, and this increases the friction force between the surface and the moving finger.

The induced friction is perceived by the mechanoreceptors in the fingertip skin. In general, mechanoreceptors are responsible to perceive sensations such as pressure, vibration, and texture, and there are four types of them in hairless skin, Merkel discs, Meissner's corpuscles, Ruffini corpuscles, and Pacinian corpuscles, as shown in Figure 2. They are categorized into fast-adapting (Pacinian and Meissner) and slow-adapting (Merkel and Ruffini) receptors. The former ones detect small and fast changes such as surface roughness, while the latter ones detect static perception such as pressure. It has been shown that the electrovibration is primarily perceived through the Pacinian channel [9].

Nevertheless, when a potential is applied, the electrostatic force, $F_{e}=\frac{\epsilon A V^{2}}{2 d^{2}}$, compresses the stratum corneum, where $A$ is contact area, $d$ is thickness of stratum corneum, $\mathrm{V}$ is instantaneous potential difference, and $\epsilon$ is dielectric constant. Because there are no nerve endings in the stratum corneum, the compression will not be sensed. By moving the skin along the metal electrode, another force perpendicular to the compressive force will arise. This frictional tangential force is given by $F_{t}=\mu\left(F_{e}+F_{n}\right)$, where $\mu$ is coefficient of friction and $F_{n}$ is contact pressure (normal force) exerted by human body. $\mu$ is therefore an appreciable amount of transfer from the perpendicular compressional force to the tangential frictional force.

This electrostatic stimulation was introduced into a tactile display by Strong et al. [10]. They developed the first electrostatic display using a stimulator array consisting of a large number of small electrodes. They reported that the intensity of the perceived vibration was mainly due to the peak applied voltage. Later, a polyimide-on-silicon electrostatic fingertip tactile display was fabricated with 49 electrodes arranged in a square array [11]. They conducted experiments to assess the intensity and spatial resolution of the tactile percepts. In a following study, its application to present various spatial tactile patterns such as line, triangle, square, and circle to the visually impaired users is investigated [12]. In all these works, the dryness of fingertip is emphasized to be the key factor maintaining the percept, reporting that a small amount of sweat could cause the percept to fade or disappear. The direct method has difficulty in stable stimulation because of finger perspiration. Indirect stimulation was suggested as a solution. Yamamoto et al. built a display with a thin slider film between electrostatic stator electrodes and fingertip

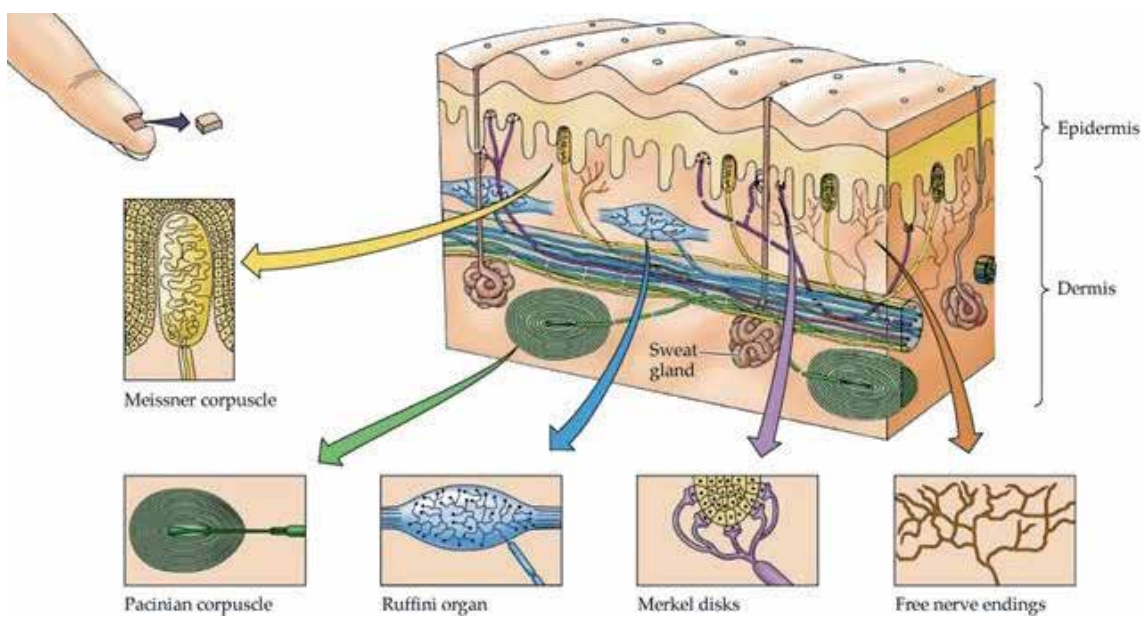

Figure 2.

Touch mechanoreceptors in the hairless (glabrous) skin of the human fingertip [8]. 
for presenting surface roughness [13]. In another work, multiple contact pads are used for multi-finger interaction with a large electrostatic display [14]. This was mainly to address finger perspiration during direct interaction and also to create larger force by applying higher voltage. This also enables multi-finger interaction.

Electrovibration regained attention in 2010 after a collaboration between Disney Research and Carnegie Mellon University yielded to a system for rendering 3D textures onto an electrovibration touchscreen. Called TeslaTouch [15], the developed system could deliver variable friction to user's sliding finger by modifying amplitude and frequency of the excitation signal. Implemented on top of a tablet computer, a user could perceive real-time tactile feedback correspond to the displayed digital content. Different tactile effects could be generated mimicking surface geometry such as bumps and ridges or surface texture such as frictional patterns to enhance user experience interacting with the objects in the scene.

The core of TeslaTouch is a transparent capacitive touch panel (Microtouch, $3 \mathrm{M}$, USA) driven by a high-voltage signal to modulate friction on a sliding finger. The panel is made of a thick glass layer on the bottom, a transparent electrode (indium tin oxide; ITO) in the middle, and a thin insulator layer on the top. In the usual setup, the electrode is excited by high AC voltage, and the human body is grounded electrically. The big advantage of TeslaTouch is that the capacitive panel is a commercial off-the-shelf product which requires only an additional high-voltage amplifier for proper operation. The same panel has been used in electrovibration displays by other groups [16-22]. Radivojevic et al. at Nokia introduced a flexible and bendable version by replacing indium tin oxide (ITO) with graphene [23].

While TeslaTouch was mainly designed for desktop applications, a company in Finland, Senseg, developed Tixel [24], a transparent electrostatic film targeting handheld devices. The touch panel is made of transparent electrodes on a glass plate coated with an insulating layer. By applying a periodic voltage to the electrodes via connections used for sensing a finger's position on the screen, the researchers were able to effectively induce a charge in a finger dragged along the surface. By changing the amplitude and frequency of the applied voltage, the surface can be made to feel as though it is bumpy, rough, sticky, or vibrating. The major difference is the specially designed control circuit that produces the sensations.

The tactile experience comes from two components: a coating layered atop touchscreen and electronics that modulate the electrostatic field and produce textures. Senseg's Tixel is the means by which Senseg's technology transmits electrovibration stimulus. It is an ultrathin durable coating on the touch interface that outputs tactile effects. The hardware inside a device modulates the signal for varied intensities of tactile sensation and types of tactile effects and provides accurate spatial resolution over the entire Tixel surface area.

Senseg later introduced a short-lived commercial product called Feelscreen, a 7" Android tablet overlaid with Tixel, into the market between 2014 and 2016. Feelscreen has been used in several projects such as 3D shape rendering [25], texture gradients [26], and visual and haptic latency [27]. At the moment, Tanvas [28], a startup company in the USA, is commercializing similar products but on a larger $10^{\prime \prime}$ tablet with some improvements such as generating stronger friction forces and not requiring an external power supply.

Some other researchers developed their own electrovibration display not using the $3 \mathrm{M}$ capacitive touch panel. Pyo et al. built a tactile display that provides both electrovibration and mechanical vibration on a large surface [29]. They fabricated an insulated ITO electrode on top of an electrostatic parallel plate actuator, both operating based on the electrostatic principle. A nontransparent electrostatic friction display was also developed in $[30,31]$ using an aluminum plate covered with a thin plastic insulator film. 
These displays do not support multi-touch or localized friction modulation, and all fingers in contact with the surface experience the same sensation. This issue was addressed by several prototypes presenting local stimulation. For example, a display panel was developed with multiple horizontal and vertical ITO electrodes in a grid enabling localized stimulation at the region where the vertical and horizontal electrodes cross each other [32]. In [14], a multi-finger electrostatic display was developed consisting of a transparent electrode and multiple contact pads on which users place their fingers. Applying different voltages to the pads and electrically grounding the transparent electrode induce different frictional stimuli to the multiple fingers.

The relationship between input signal and output friction in electrostatic friction displays is not clearly understood, and a number of studies have shown great interest in defining such relationship. Researchers have worked on this topic either by measuring friction forces using a tribometer $[16,31]$ or by estimating perceived intensities in psychophysical experiments [17,33]. For instance, Meyer et al. [16] developed a tribometer to make precise measurements of finger friction and confirmed the expected square law of frictional force to driving voltage. They also showed a linear mapping between friction and normal force, confirming the Coulombic model of dry friction. Conducting a six-value effect strength subjective index rating, Wijekoon et al. showed a significant correlation (0.8) between signal amplitude and perceived intensity but no correlation between frequency and perceived intensity [33]. In [17], participants assigned a number between 0 and 100 to the subjective friction intensity. A linear fit in log-log scale was observed in the normalized results relating applied voltage amplitude to perceived friction force intensity.

As well as fabrication, various properties of electrovibration have been investigated too. The polarity effect of the actuation signal is studied in [34], reporting that tactile sensation is more sensitive to negative than positive pulses. Meyer et al. showed an expected square law dependence of frictional force, measured by a tribometer, on actuation voltage [16]. A similar approach is taken by Vezzoli et al. to develop a model for electrovibration effect considering frequency dependence [31]. Kim et al. proposed a current control method to provide more uniform perceived intensity of electrovibration [19]. In another work and by comparing two actuation signals, it is reported that square waves are more detectable than sine waves at frequencies lower than $60 \mathrm{~Hz}$ while they are same at higher frequencies [35]. Testing three methods, amplitude modulation, adding DC offset, and their combination, Kang et al. investigated low-voltage operation of electrovibration display [22]. They showed all methods increased dynamic friction force, while only DC offset increased static friction force.

\section{Applications}

To perceive the friction force generated on an electrovibration display, one requires to drag or slide their finger over the surface. While this type of interaction is natural and intuitive for most of handheld touchscreen devices, however, it limits the range of applications can benefit from this functionality. It is worth to recall that the two key attributes of real and simulated objects are shape (surface geometry) and texture (simply surface frictional properties) [36]. Addressing these two attributes separately, in this section we review the relevant work in the literature.

\subsection{Rendering surface geometry}

Rendering 3D objects on a flat surface, either using a haptic interface or a variable friction display, has not been addressed much in the literature. In an early 
work regarding haptic perception of curvature, Gordon and Morison showed that the gradient is an effective stimulus for curvature perception and humans rely on local curvature when perceiving surface [37]. Later, Minsky et al. demonstrated that tangential force alone can be sufficient for rendering surface texture assuming it is made of little bumps [38]. They introduced gradient technique to create the illusion of bumps and valleys using a 2D force-feedback joystick. As the user moves the joystick in a direction which is up a bump, his motion is opposed by a spring force proportional to the height of the bump. This gives the sense that it is very difficult to move to the top of the bump (springs resist being stretched) and easy to fall off the bump back into a lower region of the simulated surface (springs like to revert to a short length). For fine-grained surfaces, joystick spring forces can be computed based on a local gradient. As the user moves the joystick on the virtual surface, the change in height in the direction of motion is noted. We create virtual springs opposing the motion "up" the sides of each tiny bump. Thus, the spring forces applied to the hand are computed from local gradients of the height of the surface.

Based on the gradient technique, an early attempt to create the haptic illusion of a non-flat shape on a nominally flat surface was introduced in [39] using a forceshading algorithm. Later continuing their earlier work [40], Robles-De-La-Torre and Hayward demonstrated that in active exploration of a physical shape, lateral force applied to the sliding finger plays the main role in the perception of shape [41]. They investigated the accuracy of physical shape recognition using a onedegree of freedom (DoF) force-feedback device without visual cues. Different combinations of physical and virtual geometries (bump, hole, and flat surface), e.g., a virtual bump laid on a physical flat surface, were presented to participants. The virtual shapes were rendered using lateral force only. Participants could accurately identify the virtual shapes in all conditions.

This study was foundational to the gradient-based algorithm of Kim et al. [17] for rendering 3D features on a touchscreen using electrovibration. In their work, a psychophysical perceptual model, subjectively relating the perceived friction to the applied voltage, was formulated. The model was a straight line in log-log scale, fitted over average users' ratings of the perceived friction intensity in a scale of 0-100. The model then utilized to modulate friction and render three lateral force profiles: height, slope, and rectangular. They compared users' preference for three types of force profile for a visual bump displayed on the screen. Results indicated that the slope profile best matched the visual bump. They generalized this finding to a $2 \mathrm{D}$ gradient-based rendering algorithm for 3D features and applied the algorithm to many user interface examples.

In Ref. [25], the authors presented an effective rendering method for improving the recognition of 3D features rendered on a touchscreen using an electrostatic friction display. First, a formative user study is carried out using a basic gradient-based algorithm adapted from [41] in order to assess users' ability of recognizing primitive 3D shapes based on lateral force feedback provided by an electrostatic tablet and a force-feedback interface. Experimental results demonstrated that users are not able to associate electrovibration patterns with geometric shapes in an absolute manner without contextual information. However, when such guidance was given, participants achieved moderate recognition. Then, they extended the basic algorithm to support general 3D mesh objects. The generalized algorithm computes the frictional rendering force by estimating the gradient at the touch point and also emphasizes sharp edges on the surface by rendering perceptually salient friction effects. Lastly, they conducted a summative user study to evaluate the effectiveness of their proposed shape rendering algorithm in reducing the visual uncertainty in 3D shape perception. They found that when frictional feedback was provided, the 
correct recognition performance was notably increased in comparison with when only visual rendering was presented.

\subsection{Rendering surface texture}

Compared to the problem of rendering 3D geometries on a flat electrostatic display, rendering surface textures seems more feasible and intuitive on such displays. As mentioned earlier, depending on the actuation signal, an electrovibration display generates different textural patterns. A simple illustration is given in Figure 3. On one hand, a sinusoid actuation signal creates a smooth bumpiness underneath of sliding finger. On the other hand, a square wave signal generates a rough and edgy feeling. A more complicated texture can be re-created using a proper complex signal.

Therefore, the type of input signal, its waveform, its amplitude, and its frequency components play a significant role on the generated textural patterns. Hence looking at the problem from a systematic standpoint, knowing the input-output relationship of the display is vital for this problem. As stated earlier, several efforts have been made modeling the display and drawing a relationship between the input actuation voltage and the output friction force. However, aside from the fact that the output force is somehow proportional to the squared input voltage, there exists no reliable general model covering all type of input signals across a wide range of frequencies. This suggests an alternative method, the so called data-driven texture rendering. Data-driven, or measurement-based, haptic rendering is a general approach that uses recordings from real objects to generate realistic haptic feedback in virtual environments [42, 43]. It can be either parametric- and physics-based, to optimize parameters of a predefined model, or nonparametric and generic. It is usually accompanied by a generic interpolation scheme to handle the data sets not being measured. It provides a unified framework to capture and display a diverse range of physical phenomena, while not requiring simulations of complex contact dynamics. This data-driven approach enables researchers to bypass the complex step of hand tuning a dynamic simulation of the target interaction to try to match a haptic sensation. Instead, the goal of the modeling process is to capture the output response of the system (e.g., force and acceleration) given some set of user inputs (e.g., position, velocity, and force). Such methods shift the focus from reproducing the physics of the interaction to reproducing the real sensations felt by the user, and thus they have been largely successful at realistic haptic simulation [44].

While the problem of data-driven haptic texture rendering has been fairly addressed in the literature using conventional or customized haptic interfaces
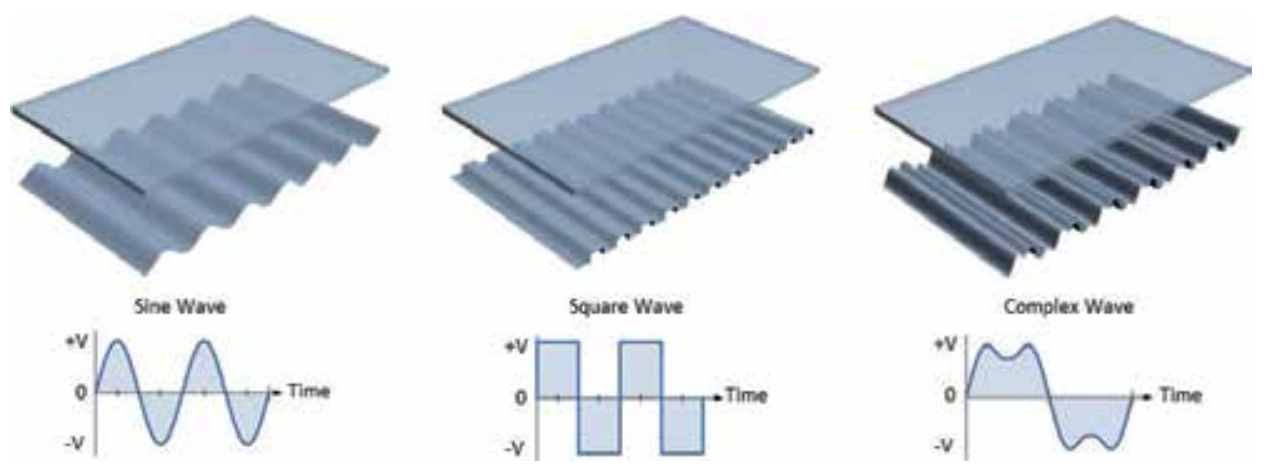

Figure 3.

How the input actuation signal makes the perceived friction different. 
[45-51], little work has been done on variable friction displays and particularly using electrovibration attraction.

An electrostatic friction display creates clearly perceptible stimuli when the surface is laterally scanned, but not when the finger is stationary. This fundamental limitation has confined the application of electrostatic friction displays mostly to texture rendering. In the only relevant work [18], Ilkhani et al. proposed a data-driven texture rendering method by recording accelerations from three real materials and playing them back on an electrovibration display. Their automated data collection is done under single constraint condition (contact force $0.35 \mathrm{~N}$ and scanning velocity $0.74 \mathrm{~m} / \mathrm{s}$ ) using a servomotor controlled by an Arduino Uno. They conducted a user study to compare the perceived surface roughness generated with their data-driven signals and with that of square wave signals. The frequency of each square wave is set based on the main frequency of the corresponding acceleration. Using a visual indicator, they made the user to keep a constant scanning velocity, but not equal to the data collection velocity and presumably very slower than that. In addition, there is no mention of contact force status during experimentation. Nevertheless, they reported higher percentage of similarity between data-driven textures and real ones than square wave patterns. In their extended work [52], they applied the same approach on the data from Penn Haptic Texture Toolkit [53] and performed MDS analysis to create a perceptual space and to extract underlying dimensions of the textures. Their results showed roughness and stickiness as the primary dimensions of texture perception.

In ref. [54], a data-driven neural network for realistic texture rendering on an electrovibration display is proposed. First, a motorized linear tribometer is developed to collect lateral frictional forces from the textured surfaces under various scanning velocities and normal forces. Then an inverse dynamics model of the display is created to describe its output-input relationship using nonlinear autoregressive with external input (NARX) neural networks. Forces resulting from applying a full-band pseudorandom binary signal (PRBS) to the display are used to train each network under the given experimental condition. A comparison between the real and virtual forces in frequency domain shows promising results and reveals the capabilities and limitations of the proposed technique.

\section{Conclusions}

In this chapter, we have introduced the concept behind electrostatic friction displays (also called electrovibration displays) and their potential applications for shape and texture rendering. The potential uses for the technique are exciting. Electrovibration could make interactive textbooks more engaging on tablets, allowing students to explore the three-dimensional features of an object directly on each page. Software for iOS or Android could be augmented with unique haptic feedback for button presses and swipe gestures. Games could incorporate electrovibration to add a new layer of interactivity to touch controls. With some smart design, it could really improve the functionality of touchscreens used in other fields, as well. For instance, the use of touchscreens in automobiles to navigate the map or control the music playback persuades drivers to avert their eyes from the road. Possibly, with an appropriate design, the same control functionalities could be delivered using a variable touch-based feedback without the need to take our eyes off the road. Given the commonness of capacitive touchscreens, the addition of richer tactile feedback through electrovibration promises to enhance almost all of our interactions with digital contents. 
While the technique has a lot of potential, the form factor remains a primary barrier to adoption. Implementing the alternating voltage results in a much bulkier device than with an ordinary capacitive touchscreen. As the technology sees more frequent use, however, there may be technological developments that allow more smartphone and tablet manufacturers to feature electrovibration without sacrificing the compactness of their designs.

\section{Acknowledgements}

I would like to thank Prof. Seungmoon Choi for his extensive personal and professional guidance teaching me a great deal about both scientific research and life in general. As my teacher and mentor, he has taught me more than I could ever give him credit for here. He has shown me, by his example, what a good scientist (and person) should be. I also would like to thank Dr. Jin Ryung Kim for his constant support and encouragement.

\section{Conflict of interest}

I certify that I have no affiliations with or involvement in any organization or entity with any financial interest (such as honoraria; educational grants; participation in speakers' bureaus; membership, employment, consultancies, stock ownership, or other equity interest; and expert testimony or patent-licensing arrangements) or nonfinancial interest (such as personal or professional relationships, affiliations, knowledge, or beliefs) in the subject matter or materials discussed in this manuscript.

\section{Author details}

Reza Haghighi Osgouei

Imperial College London, London, UK

*Address all correspondence to: r.haghighi-osgouei@imperial.ac.uk

IntechOpen

(C) 2020 The Author(s). Licensee IntechOpen. This chapter is distributed under the terms of the Creative Commons Attribution License (http://creativecommons.org/licenses/ by/3.0), which permits unrestricted use, distribution, and reproduction in any medium, provided the original work is properly cited. (cc) BY 


\section{References}

[1] Haghighi Osgouei R. Haptic Rendering of Surface 3D Curvature and Texture on Electrostatic Friction Display. 2017

[2] Haghighi Osgouei R, Kim JR, Choi S. Identification of primitive geometrical shapes rendered using electrostatic friction display. In: IEEE Haptics Symposium (HAPTICS). 2016. pp. 198-204

[3] Gray E, inventor. Improvement in electric telegraphs for transmitting musical, tones. United States patent US US166,096; 1875

[4] Haghighi Osgouei R, Kim JR, Choi S. Data-driven texture modeling and rendering on electrovibration display. IEEE Transactions on Haptics. 2019. DOI: 10.1109/TOH.2019.2932990

[5] Johnsen A, Rahbek K. A physical phenomenon and its applications to telegraphy, telephony, etc. Journal of the Institution of Electrical Engineers. 1923;61(320):713-725

[6] Mallinckrodt E, Hughes AL, Sleator W Jr. Perception by the skin of electrically induced vibrations. Science. 1953;118:277-278. Available from: https://doi.org/10.1126/ science.118.3062.277

[7] Grimnes S. Electrovibration, cutaneous sensation of microampere current. Acta Physiologica

Scandinavica. 1983;118(1):19-25

[8] Darian-Smith I. The sense of touch: Performance and peripheral neural processes. In: Comprehensive Physiology. Wiley [Online Library]. 2011. pp. 739-788

[9] Vardar Y, Güçlü B, Basdogan C.

Effect of waveform on tactile perception by electrovibration displayed on touch screens. IEEE Transactions on Haptics. 2017;10(4):488-499
[10] Strong RM, Troxel DE. An electrotactile display. IEEE Transactions on Man-Machine Systems. 1970;11(1): 72-79

[11] Beebe DJ, Hymel CM, Kaczmarek KA, Tyler ME. A polyimideon-silicon electrostatic fingertip tactile display. In: Engineering in Medicine and Biology Society, 1995, IEEE 17th Annual Conference. Vol. 2. 1995. pp. 1545-1546

[12] Tang H, Beebe DJ. A microfabricated electrostatic haptic display for persons with visual impairments. IEEE Transactions on Rehabilitation Engineering. 1998;6(3): 241-248

[13] Yamamoto A, Ishii T, Higuchi T. Electrostatic tactile display for presenting surface roughness sensation. In: IEEE International Conference on Industrial Technology. Vol. 2. 2003. pp. 680-684

[14] Nakamura T, Yamamoto A. Multifinger electrostatic passive haptic feedback on a visual display. In: World Haptics Conference (WHC). 2013. pp. $37-42$

[15] Bau O, Poupyrev I, Israr A, Harrison C. TeslaTouch: Electrovibration for touch surfaces. In: Proceedings of the 23nd Annual ACM Symposium on User Interface Software and Technology. 2010. pp. 283-292

[16] Meyer DJ, Peshkin MA, Colgate JE. Fingertip friction modulation due to electrostatic attraction. In: World Haptics Conference (WHC). 2013. pp. 43-48

[17] Kim SC, Israr A, Poupyrev I. Tactile rendering of $3 \mathrm{D}$ features on touch surfaces. In: Proceedings of the 26th Annual ACM Symposium on User Interface Software and Technology. 2013. pp. 531-538 
[18] Ilkhani G, Aziziaghdam M, Samur E. Data-driven texture rendering with electrostatic attraction. In: International Conference on Human Haptic Sensing and Touch Enabled Computer Applications. 2014. pp. $496-504$

[19] Kim H, Kang J, Kim KD, Lim KM, Ryu J. Method for providing electrovibration with uniform intensity. IEEE Transactions on Haptics.

2015;8(4):492-496

[20] Zhang Y, Harrison C. Quantifying the targeting performance benefit of electrostatic haptic feedback on touchscreens. In: Proceedings of the International Conference on Interactive Tabletops \& Surfaces. 2015. pp. 43-46

[21] Wang Q, Ren X, Sarcar S, Sun X. EV-pen: Leveraging Electrovibration haptic feedback in pen interaction. In: Proceedings of the ACM on Interactive Surfaces and Spaces. 2016. pp. 57-66

[22] Kang J, Kim H, Choi S, Kim KD, Ryu J. Investigation on low voltage operation of electrovibration display. IEEE Transactions on Haptics. 2017;10(3):371-381

[23] Radivojevic Z, Beecher P, Bower C, Haque S, Andrew P, Hasan T, et al. Electrotactile touch surface by using transparent graphene. In: Proceedings of the Virtual Reality International Conference. 2012. p. 16

[24] Linjama J, Mäkinen V. E-sense screen: Novel haptic display with capacitive electrosensory interface. In: HAID, 4th Workshop for Haptic and Audio Interaction Design. 2009

[25] Haghighi Osgouei R, Kim JR, Choi S. Improving 3D shape recognition with electrostatic friction display. IEEE Transactions on Haptics. 2017;10(4):533-544

[26] Klatzky RL, Adkins S, Bodas P, Haghighi Osgouei R, Choi S, Tan HZ.
Perceiving texture gradients on an electrostatic friction display. In: World Haptics Conference (WHC). 2017. pp. $154-158$

[27] Kim JR, Haghighi Osgouei R, Choi S. Effects of visual and haptic latency on touchscreen interaction: A case study using painting task. In: World Haptics Conference (WHC). 2017. pp. $159-164$

[28] TanvasTouch ${ }^{\circledR}$. Available from: https://tanvas.co [Accessed: 02 September 2020]

[29] Pyo D, Ryu S, Kim SC, Kwon DS. A new surface display for 3D haptic rendering. In: International Conference on Human Haptic Sensing and Touch Enabled Computer Applications. 2014. pp. 487-495

[30] Giraud F, Amberg M, LemaireSemail B. Merging two tactile stimulation principles: Electrovibration and squeeze film effect. In World Haptics Conference (WHC). 2013. pp. 199-203

[31] Vezzoli E, Amberg M, Giraud F, Lemaire-Semail B. Electrovibration modeling analysis. In International Conference on Human Haptic Sensing and Touch Enabled Computer Applications. 2014. pp. 369-376

[32] Haga H, Yoshinaga K, Yanase J, Sugimoto D, Takatori K, Asada H. Electrostatic tactile display using beat phenomenon of voltage waveforms. In: SID Symposium Digest of Technical Papers. Vol. 45. No. 1. 2014. pp. 623-626

[33] Wijekoon D, Cecchinato ME, Hoggan E, Linjama J. Electrostatic modulated friction as tactile feedback: Intensity perception. In: International Conference on Human Haptic Sensing and Touch Enabled Computer Applications. 2012. pp. 613-624

[34] Kaczmarek KA, Nammi K, Agarwal AK, Tyler ME, Haase SJ, 
Beebe DJ. Polarity effect in electrovibration for tactile display. IEEE Transactions on Biomedical Engineering. 2006;53(10):2047-2054

[35] Vardar Y, Güçlü B, Basdogan C. Effect of waveform in haptic perception of electrovibration on touchscreens. In: International Conference on Human Haptic Sensing and Touch Enabled Computer Applications. 2016. pp. 190-203

[36] Campion G, Hayward V. Fundamental limits in the rendering of virtual haptic textures. In: First Joint Eurohaptics Conference and Symposium on Haptic Interfaces for Virtual Environment and Teleoperator Systems. World Haptics Conference. Pisa, Italy; 2005. pp. 263-270

[37] Gordon IE, Morison V. The haptic perception of curvature. Perception \& Psychophysics. 1982;31(5):446-450

[38] Minsky M, Ming OY, Steele O, Brooks Jr FP, Behensky M. Feeling and seeing: Issues in force display. In: Proceedings of the 1990 Symposium on Interactive 3D Graphics (I3D '90). Association for Computing Machinery. New York, NY, USA; 1990:235-241. Available from: https://doi.org/10.1145/91385.91451

[39] Morganbesser HB, Srinivasan MA. Force shading for shape perception in haptic virtual environments. In: Proceedings of the 5th Annual Symposium on Haptic Interfaces for Virtual Environment and Teleoperator Systems. Vol. 58. Atlanta, GA, DSC: ASME/IMECE; 1996

[40] Robles-De-La-Torre G, Hayward V. Virtual surfaces and haptic shape perception. In: Proceedings ASME IMECE Symposium on Haptic Interfaces for Virtual Environments and Teleoperator Systems. Vol. 69. 2000. p. 2

[41] Robles-De-La-Torre G, Hayward V. Force can overcome object geometry in the perception of shape through active touch. Nature. 2001;412(6845):445

[42] Hover R, Kósa G, Szekly G, Harders M. Data-driven haptic rendering-From viscous fluids to visco-elastic solids. IEEE Transactions on Haptics. 2009;2(1):15-27

[43] Yim S, Jeon S, Choi S. Data-driven haptic modeling and rendering of viscoelastic and frictional responses of deformable objects. IEEE Transactions on Haptics. 2016;9 (4):548-559

[44] Romano JM, Yoshioka T, Kuchenbecker KJ. Automatic filter design for synthesis of haptic textures from recorded acceleration data. In: IEEE International Conference on Robotics and Automation (ICRA). 2010. pp. $1815-1821$

[45] Choi S, Tan HZ. Toward realistic haptic rendering of surface textures. IEEE Computer Graphics and Applications. 2004;24(2):40-47

[46] Andrews S, Lang J. Haptic texturing based on real-world samples. In: IEEE International Workshop on Haptic, Audio and Visual Environments and Games (HAVE). 2007. pp. 142-147

[47] Campion G. On the synthesis of haptic textures. In: The Synthesis of Three Dimensional Haptic Textures: Geometry, Control, and Psychophysics. London: Springer; 2008. pp. 73-97

[48] Lang J, Andrews S. Measurementbased modeling of contact forces and textures for haptic rendering. IEEE Transactions on Visualization and Computer Graphics. 2011;17(3):380-391

[49] Culbertson H, Unwin J, Kuchenbecker KJ. Modeling and rendering realistic textures from unconstrained tool-surface interactions. IEEE Transactions on Haptics. 2014;3:1

[50] Shin S, Haghighi Osgouei R, Kim KD, Choi S. Data-driven modeling 
of isotropic haptic textures using frequency-decomposed neural networks. In: World Haptics Conference (WHC). 2015. pp. 131-138

[51] Abdulali A, Jeon S. Data-driven rendering of anisotropic haptic textures. In: International Asia Haptics Conference. 2016. pp. 401-407

[52] Ilkhani G, Aziziaghdam M, Samur E. Data-driven texture rendering on an electrostatic tactile display. International Journal of Human-Computer Interaction. 2017;33(9):756-770

[53] Culbertson H, Lopez Delgado JJ, Kuchenbecker KJ. The Penn Haptic Texture Toolkit for modeling, rendering, and evaluating haptic virtual textures, Departmental Papers (MEAM). 299; 2014

[54] Haghighi Osgouei R, Shin S, Kim JR, Choi S. An inverse neural network model for data-driven texture rendering on electrovibration display. In: IEEE Haptics Symposium (HAPTICS). 2018. pp. 270-277 



\title{
Failure Mechanism and Property Modification of Insulation Material Used in Inverter-Fed Motors
}

\author{
Yan Yang and Guangning Wu
}

\begin{abstract}
The insulation system of inverter-fed motors is subjected to repetitive impulse voltages, which are generated by pulse width modulation (PWM) converters, and is expected to withstand partial discharge (PD) activity during service. PD-induced dielectric breakdown becomes one of the most important reasons for premature failures of motor insulation. Therefore, the dielectric properties of insulation material used in inverter-fed motors need to be improved to meet the requirement for rapid development of applications. Many approaches have been proposed to improve dielectric properties of insulation material used in inverter-fed motors. In this chapter, we will discuss the failure mechanism, modification approaches, and their effects on dielectric properties of insulation material of inverter-fed motors.
\end{abstract}

Keywords: inverter-fed motors, insulation material, failure mechanism, modification

\section{Introduction}

The insulation system of inverter-fed motors is subjected to repetitive impulse voltages that are generated by pulse width modulation (PWM) converters. For high-voltage motors with the rated voltage value greater than $700 \mathrm{~V}$, which also be called as Type II motors, partial discharges (PD) may occur in all insulation systems during operation, but breakdown due to high-voltage endurance may not occur. The insulation materials are expected to withstand partial discharge (PD) activity during service. Under repetitive impulse voltages, accelerating of insulation deterioration would be caused [1]. PD-induced dielectric breakdown is one of the most important reasons for premature failures of motor insulation [2, 3]. In inverter-fed motors, most insulation failures are originated from the non-homogeneous distribution of voltage along the stator windings and are closely related with the short rise time and high frequency of the repetitive impulse voltages [4].

Polyimide (PI) is used as an insulating material for turn-to-turn insulation of inverter-fed traction motors because of its excellent electrical, thermal, and mechanical characteristics $[1,5]$. Therefore, the dielectric properties of PI films still need to be improved to meet the requirement for rapid development of industry application [6, 7], especially for PD-dependent lifetime under repetitive impulse voltages. Many approaches have been proposed to improve PD-dependent lifetime of 
PI films. One effective approach is synthesizing PI/inorganic nanocomposites [8-11]. Polymer nanocomposites consisting of nanoparticles allow for a large tunability of the dielectric properties by varying the identity and content of nanoadditives. Moreover, lifetime under repetitive impulse voltages is much more closely related to the surface characteristics of PI films, for the degradation is always initiated from surface partial discharge, which is mainly caused by surface charge accumulation and local field enhancement, due to the mismatching of surface charge dissipation and voltage reversion [12-14]. Therefore, surface modification would be another effective approach to improve the lifetime of PI films under repetitive impulse voltages, by modifying the surface characteristics.

To provide a comprehensive understanding to this topic and to clarify the emerging problems, we review the recent progress in the failure mechanism and property modification of insulation material used in inverter-fed motors. Particular attention is paid on PD-dependent lifetime improvement under repetitive impulse voltages of PI films.

\section{Failure mechanism of insulation material used in inverter-fed motors}

\subsection{Electrical stresses in inverter-fed motors}

A typical schematic diagram and output waveform of inverter-fed traction motors used in high-speed railways are shown in Figure 1 [5]. Different from traditional alternating current (AC) motors stressed by the sinusoidal $(50$ or $60 \mathrm{~Hz}$ ) voltages, the insulation systems of inverter-fed motors are exposed to transient surge voltages with steep fronts generated by the switching of insulated gate bipolar transistor (IGBT) [6]. Besides, there are other factors that cause PDs, including overvoltage caused by impedance mismatch (between supply cable and motor) and uneven potential distribution could induce partial discharge (PD) in stator

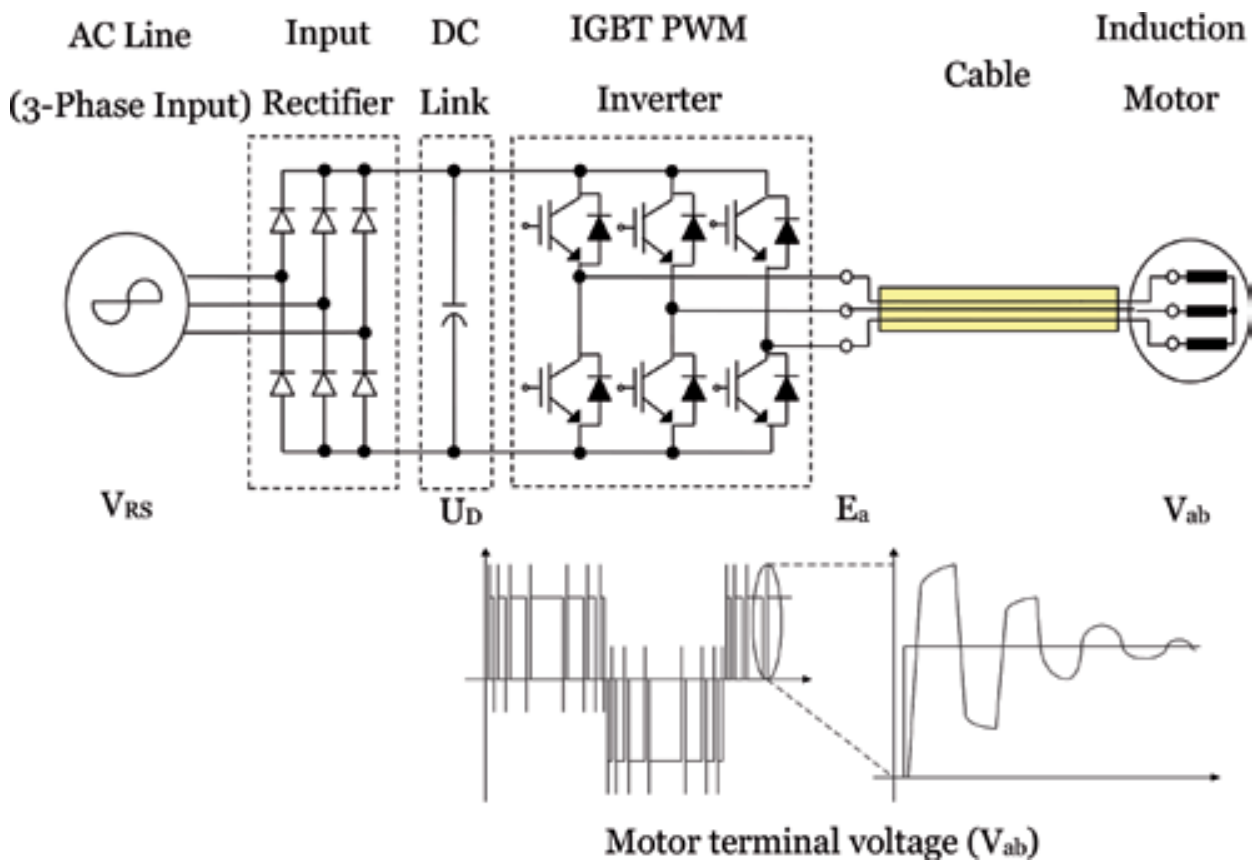

Figure 1.

Schematic diagram and output waveform of an inverter [5]. 

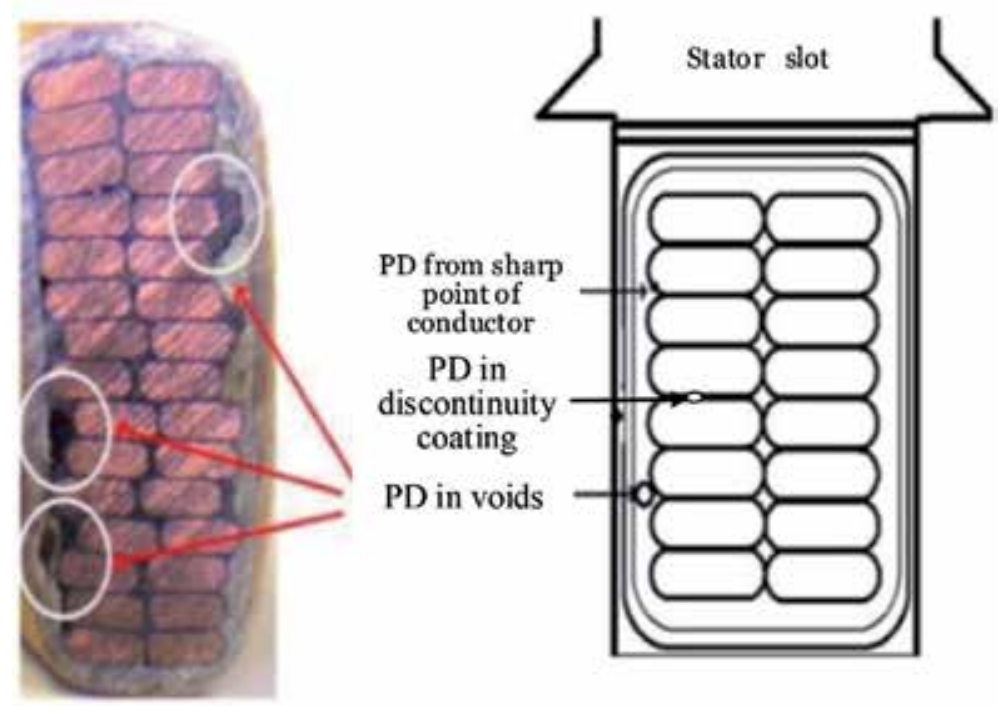

Figure 2.

Typical PD positions in the inner stator slot of an inter-fed motor [18].

insulation systems [15-17]. For Type II motors, formed windings are used in which the preformed coils are uniformly layered with rectangular section conductors. Form the typical cross section image of stators slot as shown in Figure 2 [18], it can be inferred that PDs are easily occur from the sharp point of conductor, discontinuity coating, and voids formed during manufacture.

\subsection{Failure mechanism under repetitive impulse voltage}

Hereinafter, we will explain failure mechanism under repetitive impulse voltages though the investigation of the aging process and PD characteristics of turn-to-turn insulation of coils under repetitive impulse voltages [5]. In order to simulate the insulation structure of coils of an inverter-fed motor, the turn insulation specimens were manufactured according to the standard IEC 60034-18-42. In laboratory, bipolar repetitive square impulse voltages are used to take the place of pulse width modulated (PWM) voltage, with adjustable parameters such as amplitude, repetition frequency, and rising time. As bipolar repetitive square impulse voltages were adopted as the testing voltage, one cycle of test time is divided into $360^{\circ}$, and thus, we can analyze $\mathrm{PD}$ signals as they can be measured under AC power frequency voltage.

PD characteristics of turn insulation specimens were investigated, with the specimens be aged for 20,60, and $100 \mathrm{~h}$, respectively. Phase distribution of the discharge numbers and amplitude of PD pulses is shown in Figure 3 [5]. Most of PD pulses appear next to the rising edge and the falling edge of square wave with a narrow distribution range from about 3 to $23^{\circ}$ and 180 to $200^{\circ}$. PD pulses are rarely present during the flat region of repetitive square impulse voltages. This phase distribution characteristic is quite different from the PD characteristics under power frequency. The phase distribution appears widening tendency with the increase of aging time. Especially for the phase near $20^{\circ}$, the PD number and PD quantity increase obviously.

Storage effect of charges is obvious under repetitive impulse voltages. Figure 4 [5] describes the change of space charge and electric field in the air gap at different time of bipolar continuous square impulse voltage. $E(t)$ is the applied field with electric field amplitude $E_{0} . E_{\mathrm{q}}$ is the electric field induced by space charge deposited 


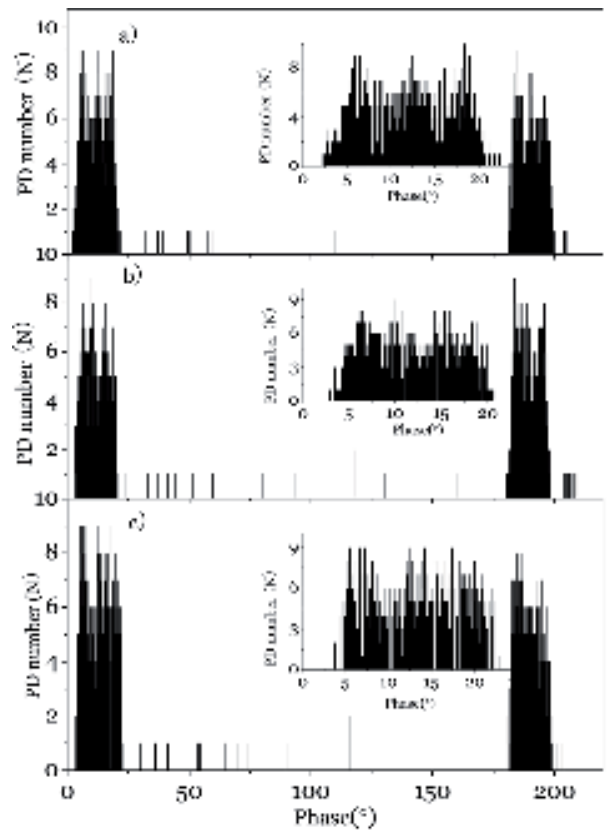

(a)

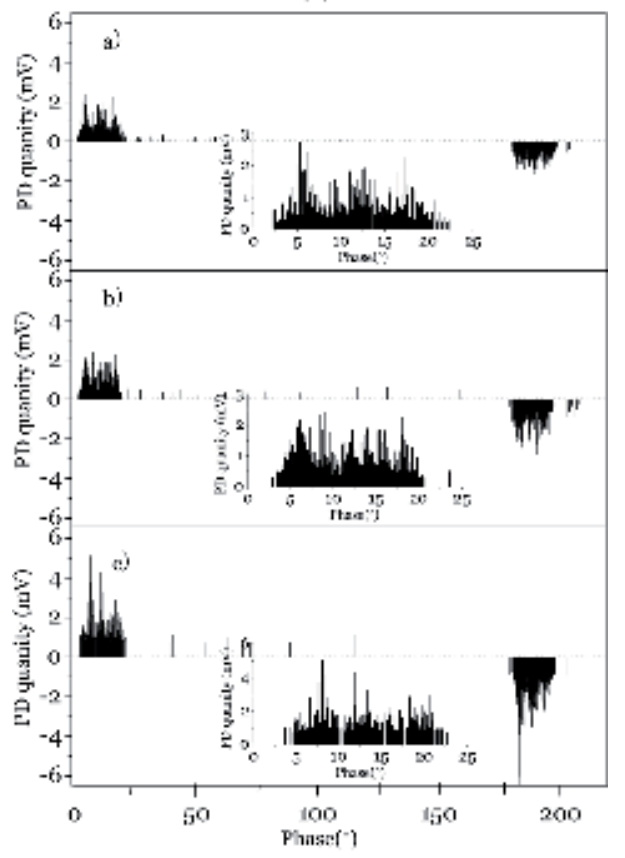

(h)

Figure 3.

$P D$ characteristics of turn insulation specimens with different aging time. (a) Relevant phase distribution of the PD number. (b) Relevant phase distribution of PD quantity [5].

in the air gap. At the beginning, bipolar continuous square impulse voltage is applied on the sample, and the induced space charges gather in the air gap, as shown in Figure 4a. If the field $E_{\mathrm{i}}$, difference between $E(t)$ and $E_{\mathrm{q}}$, reaches PD inception field and the initiating electron is available, PD occurs, dropping the electric field $E_{\mathrm{i}}$ to a residual field value Eres = E0- Eq, as shown in Figure $\mathbf{4 b}$. When the polarity of impulse voltage reverses, the field value in the air gap changes to $\mathrm{Ei}=-(\mathrm{E}(\mathrm{t})+\mathrm{Eq})$, as shown in Figure 4c. Currently, space charge moves under the inverted electric 


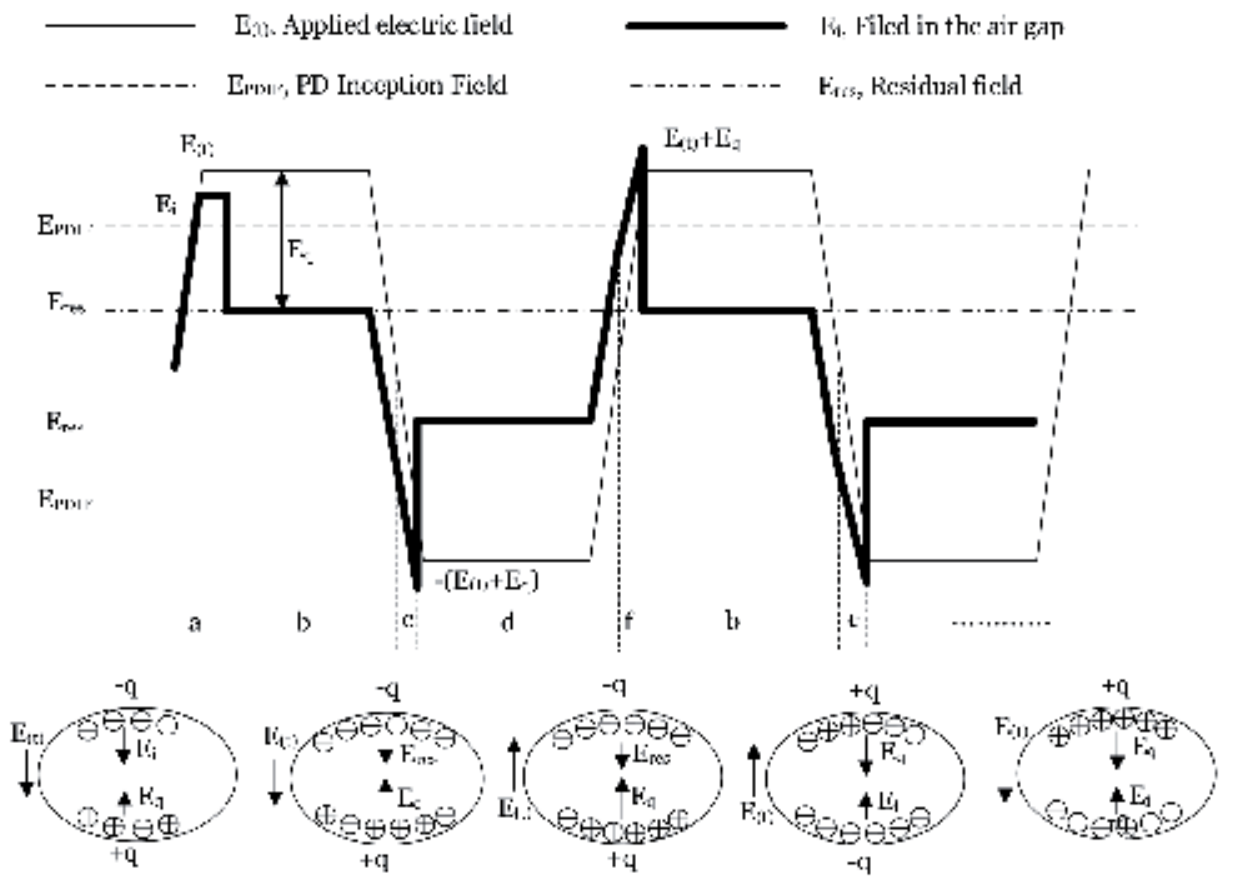

(a) boliore brealidown

(h) after breakdown

(c) polarity inwersion

(d) after bueakilown (li polarily intersion

Figure 4 .

Storage effect of charges under repetitive impulse voltages [5].

field. But this process is very weak and most of the charge cannot perform the invert motion because of the steep front of impulse voltage. The value of $E_{\mathrm{q}}$ decreases only a little, where $\mathrm{Eq} \approx \mathrm{E} 0$-Eres. So, at the instant of polarity reverses, $\mathrm{Ei} \approx(\mathrm{E}(\mathrm{t})+\mathrm{E} 0$-Eres $)$ would be larger than PD inception field, another PD could be promoted at the falling time of bipolar continuous square impulse voltage, as shown in Figure 4c. The charge in the air gap completes reverse movement during the process of PD and the electric field Ei drops again to the value -Eres, as shown in Figure 4d. When the applied field inverse again, which is shown in Figure 4f, the changes of charge and electric field Ei in the air are similar with that in Figure 4c. PD pulse would appear again. The behavior of charge and field could repeat in succession with the testing time, which is similar with the procedures from step $b$ to step $f$.

As displayed in Figure 4, the electric field $\mathrm{Ei}$ in the air gap reaches to the maximum value at the rising stage and falling stage of bipolar continuous square impulse voltage. The main reason is that the movement of charge in the air gap could not follow the polarity reversion of impulse voltage; superposition effect between applied field $\mathrm{E}(\mathrm{t})$ and the field $\mathrm{Eq}$ in the air gap is obvious. Consequently, Most of PDs occur during the rising edge and falling edge of continuous square impulse voltage with a narrow distribution range, which can be observed in Figure 3. Residual field value Eres is not constant strictly because of the dissipation of charge. Individual PD appears during the flat region of bipolar square impulse voltage when the field Ei increases to PD inception filed accidentally because of the dissipation of charge in the air gap.

Partial discharge behavior is closely related to the degree of insulation aging. During PD activities, the organic polymer is eroded and degraded gradually, which could lead to two results: first, more new voids generate, which promote the increase of PD number, and second, the original voids were enlarged because of volatilization of organics, resulting in higher PDIV. When the polarity of bipolar 
continuous square impulse voltage inverses, applied voltage needs a longer delayed time to reach the higher PDIV, resulting in a higher energy of PD, which will be appear at the phase around $20^{\circ}$ due to the time delay. This is consistent with what we discussed before that PD amplitudes and discharge numbers become higher near $20^{\circ}$ in aged samples. Therefore, PD amplitudes and discharge numbers near $20^{\circ}$ can be considered as critical characteristic parameters to evaluate the insulation condition under repetitive impulse voltages.

\section{Property modification of polyimide films}

\subsection{Polyimide nanocomposites}

Samples of $\mathrm{PI} / \mathrm{Al}_{2} \mathrm{O}_{3}$ nanocomposites are usually synthetized by using in-situ polymerization. Corona-resistance lifetime under repetitive impulse voltages for nanocomposites with different content of $\mathrm{Al}_{2} \mathrm{O}_{3}$ nanofillers is investigated. And then, the effects of nanoparticles on reducing the degradation of polyimide nanocomposites will be analyzed in this section.

\subsubsection{Lifetime under repetitive impulse voltages for polyimide nanocomposites}

During lifetime measurement under repetitive impulse voltages, PDs occur in the air gap between the film sample and the upper rod electrode. PDs generate highenergy electron, ultraviolet rays, and high activity chemical groups, which would accelerate the insulation material aging $[19,20]$. Reaching the lifetime, the failure of polymer nanocomposites would occur due to the fracture and degradation of the polymer chains [21].

As shown in Figure 5 [22], the lifetime increases with the increasing nano- $\mathrm{Al}_{2} \mathrm{O}_{3}$ content. For the $\mathrm{PI} / \mathrm{Al}_{2} \mathrm{O}_{3}$ nanocomposites with $25 \mathrm{wt}$ \% content of nano- $\mathrm{Al}_{2} \mathrm{O}_{3}$, the lifetime is around $136 \mathrm{~h}$, which is about 23 times longer than that of pure PI film, at

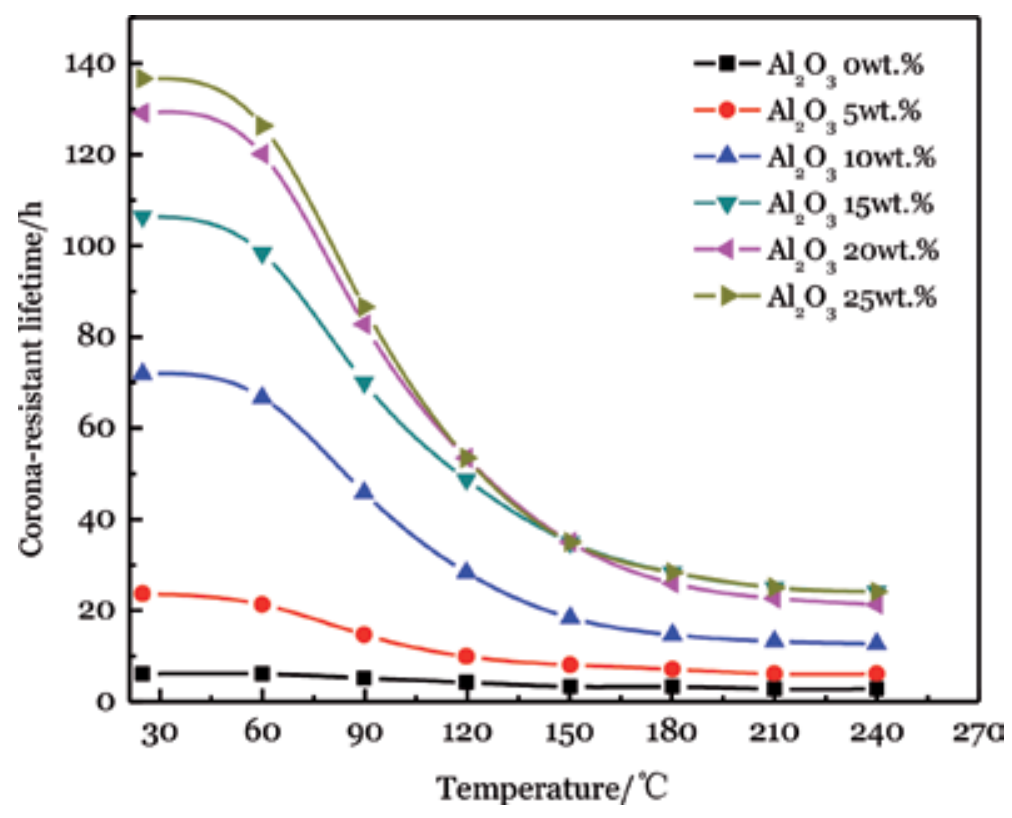

Figure 5.

Lifetime under repetitive impulse voltages of $\mathrm{PI} / \mathrm{Al}_{2} \mathrm{O}_{3}$ nanocomposites [22]. 
the temperature of $25^{\circ} \mathrm{C}$. Besides, the lifetime of $\mathrm{PI} / \mathrm{Al}_{2} \mathrm{O}_{3}$ nanocomposites exhibits the obvious temperature dependence, which decreases gradually with increasing temperature. With increasing nano- $\mathrm{Al}_{2} \mathrm{O}_{3}$ content, the temperature dependence is much stronger.

\subsubsection{Effects of nanoparticles on reducing the degradation of polyimide nanocomposites}

The effects of nanoparticles on reducing the degradation of polyimide nanocomposites can be deriving from the following three aspects.

First, during the lifetime measurement under repetitive impulse voltages, PD-induced degradation of the polymer chains occurs. With higher melting point, inorganic particles would leave on the film surface, acting as obstacles in the erosion path of polyimide matrix. Then, the degradation of polymer matrix can be reduced, resulting in a prolonged lifetime. This statement can be proved through the element distribution analysis around the breakdown point, as shown in Figure 6 and Table 1 [23]. Next to the breakdown point, the mass percentage of $\mathrm{Al}$ is almost twice higher than the mean content (2.65\%) in $\mathrm{PI} / \mathrm{Al}_{2} \mathrm{O}_{3}$ nanocomposites with $\mathrm{Al}_{2} \mathrm{O}_{3}$ content of $5 \mathrm{wt} . \%$. This is consistent with the statement that inorganic particles left on the surface of eroded regions.

Second, new chemistry bonds would be generated in the interfacial regions of polyimide nanocomposites, which is the lowest bonding energy. Under the effect of PDs, these weak bonds would be broken first, followed by the ether linkage, imide ring and aromatic ring of polyimide matrix, as shown in Figure 7 [23].

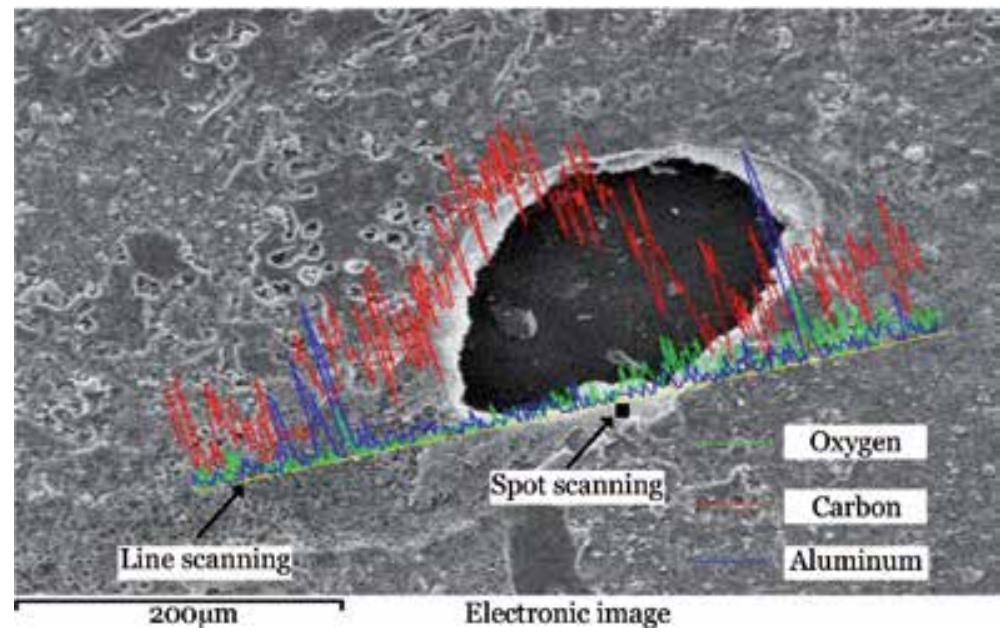

Figure 6.

EDS of spot and line scanning around the breakdown point of $\mathrm{PI} / \mathrm{Al}_{2} \mathrm{O}_{3}$ nanocomposites with $\mathrm{Al}_{2} \mathrm{O}_{3}$ content of $5 w t . \%[23]$.

\begin{tabular}{lcc}
\hline Element & Spot scanning (Mass \%) & Line scanning (Mass \%) \\
\hline Carbon & 30.61 & 29.70 \\
\hline Oxygen & 65.65 & 57.27 \\
\hline Aluminum & 5.74 & 13.03 \\
\hline
\end{tabular}

Table 1.

Mass percentage of elements in EDS result [23]. 


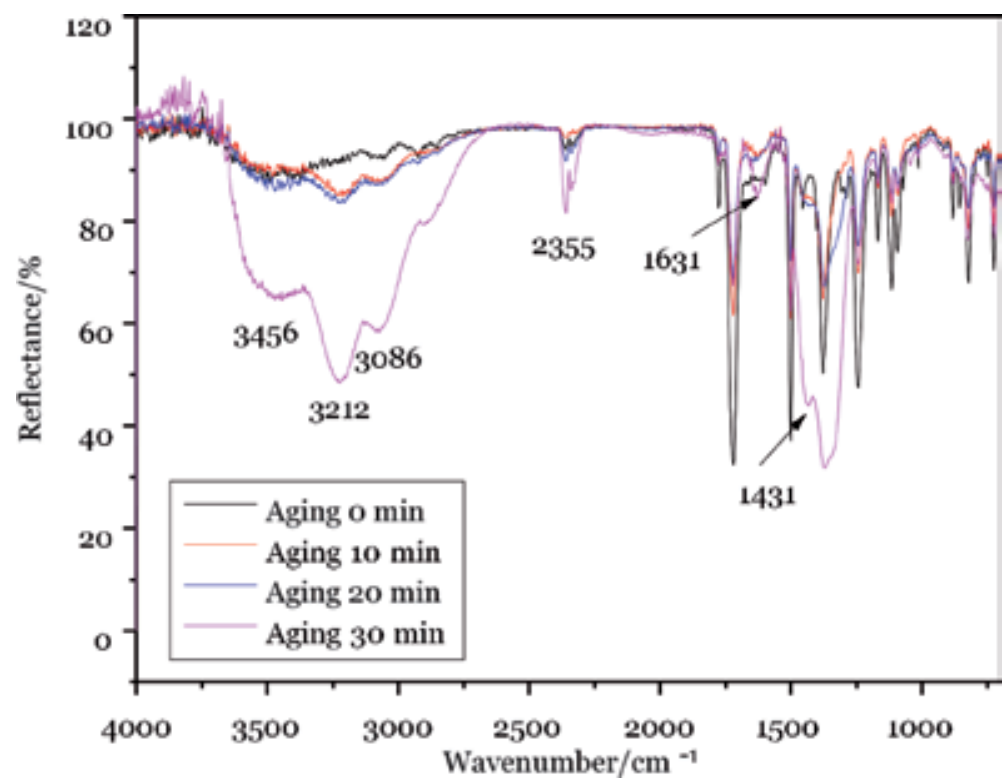

Figure 7.

FTIR spectrums of PI and PI/Al $\mathrm{O}_{3}$ nanocomposites (5 wt.\%) at different aging time [23].

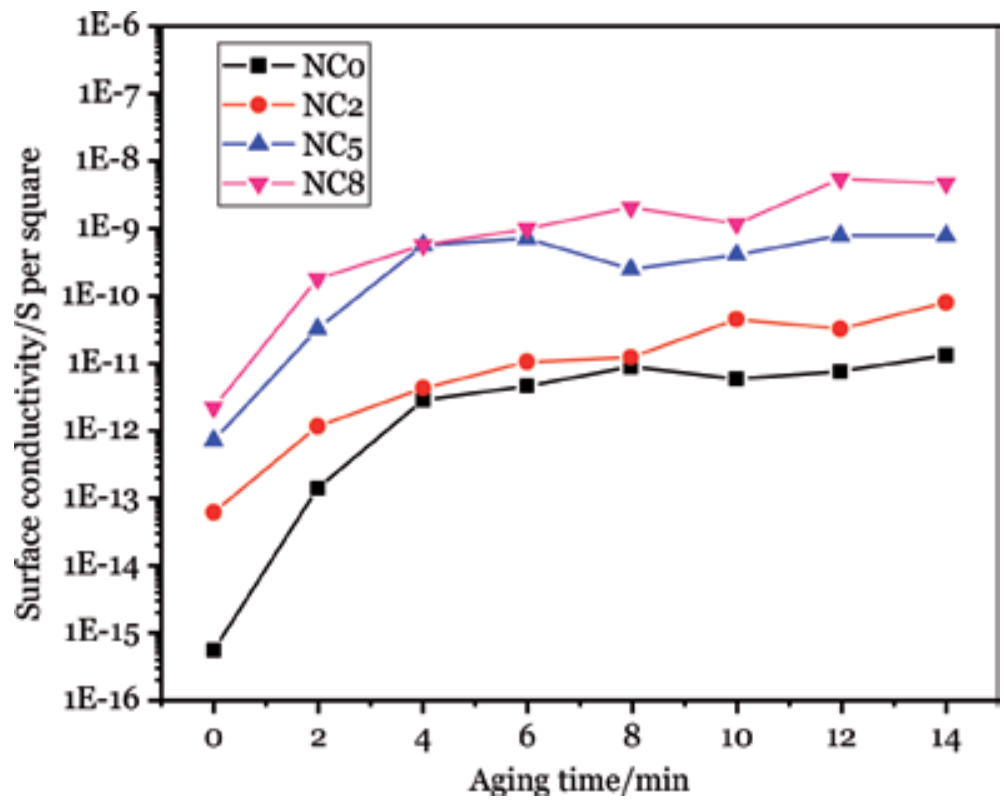

Figure 8.

Surface conductivity of $\mathrm{PI}$ and $\mathrm{PI} / \mathrm{Al}_{2} \mathrm{O}_{3}$ nanocomposites [23].

Thus, weakest bonds in interfacial regions would be destroyed at the first stage of aging, which helped reduce the dissociations of other chemical bonds of polyimide molecules, by absorbing part of PD energy, resulting in a prolonged lifetime.

Third, surface conductivity of polyimide nanocomposites is higher than that of polyimide matrix, as shown in Figure 8 [23], which facilitates surface charge dissipation, leading to lower PD intensity, and longer lifetime. The inorganic particles and the interfacial region near the film surface, with higher carrier 


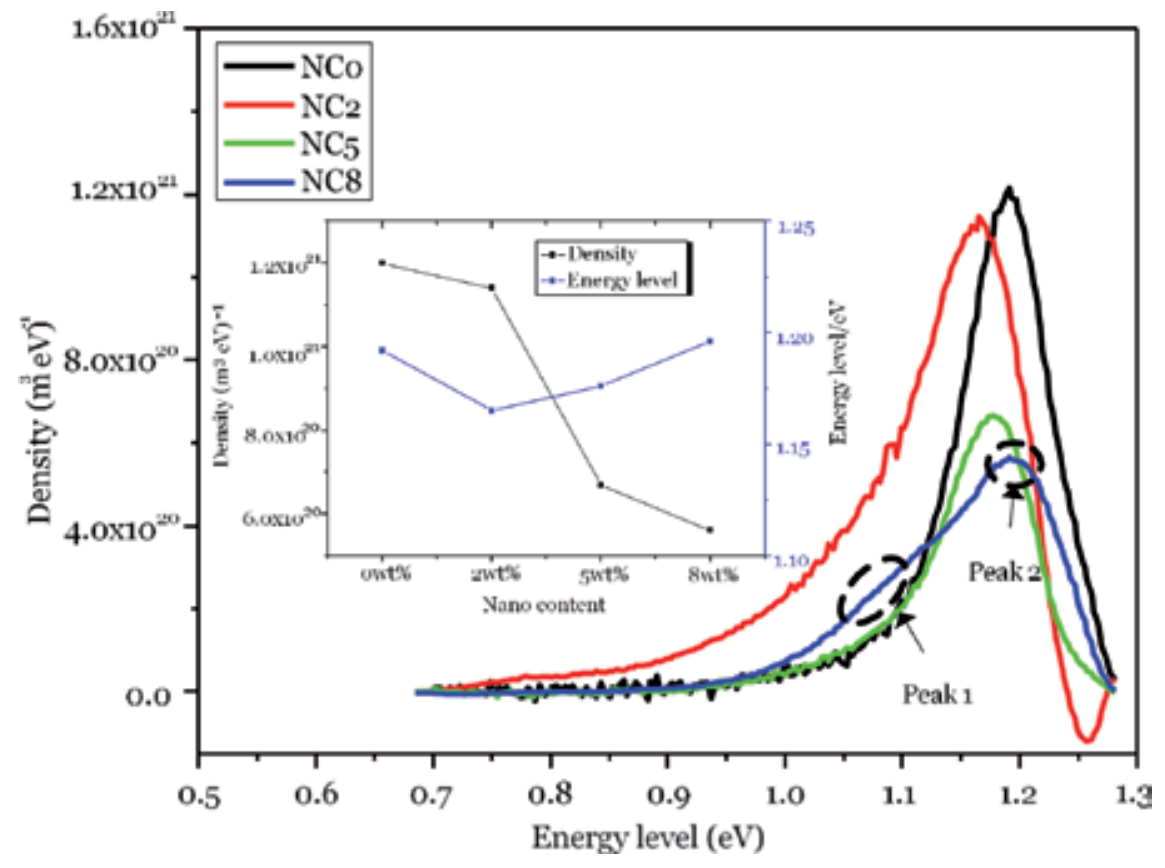

Figure 9.

Trap energy and density distributions of $\mathrm{PI}$ and $\mathrm{PI} / \mathrm{Al}_{2} \mathrm{O}_{3}$ nanocomposites [23].

transfer ability, are mainly responsible for the increase in surface conductivity of polyimide nanocomposites. Improved surface conductivity can facilitate charge dissipation and mitigates the electric field distortion, resulting in higher PDIV and longer lifetime.

At last, dielectric characteristics of polyimide nanocomposites are greatly influenced by different trap density and energy distributions which depend on physical and chemical defects in dielectrics. As to deep traps, named as peak 2 in Figure 9 [23], the trap energy nearly changes for polyimide nanocomposites, but trap density declines with increasing nanoparticle concentration, which means that the bonds between nanoparticles and polyimide molecules can restrict the movement of molecular and thus reduce the chemical disorder.

Besides, shallower traps are introduced in nanocomposites as nanocomposites content increases, as peak 1 appears on the curve of polyimide nanocomposites. With lower trap density, it is less possible for charge recombination in polyimide nanocomposites, thus lead to lower released energy, which is beneficial to reduce the degradation of polymer.

Many chemical defects are generated during PD aging due to the breakage of long molecular chain, which are reflected on the increase of both trap density and energy level. As the degree of electrical aging can be determined by the rate of increase in the density of stress-created traps [24], it can be concluded that the degree of PD aging is quite serious in PI after aging for $15 \mathrm{~min}$, while that of PI/ $\mathrm{Al}_{2} \mathrm{O}_{3}$ nanocomposites is much slighter. That is why no obvious increase trend can be observed in the trap density when the PD aging time is less than $15 \mathrm{~min}$.

Besides, the trap-level distribution showed that both trap density and energy in $\mathrm{PI} / \mathrm{Al}_{2} \mathrm{O}_{3}$ nanocomposites decreased as degradation, while the trend of PI was opposite. It indicated that the degradation of $\mathrm{PI} / \mathrm{Al}_{2} \mathrm{O}_{3}$ nanocomposites was mitigated compared with $\mathrm{PI}$, which could be attributed to the improved electrical characteristics of $\mathrm{PI} / \mathrm{Al}_{2} \mathrm{O}_{3}$ nanocomposites. 


\subsection{Surface modification of polyimide films}

Non-thermal plasma generated by dielectric barrier discharge (DBD) can be used to conduct surface modification of polyimide films [25]. This kind of plasma modification can efficiently cause the material surface cross-linking, etching, and the introduction of polar groups, without changing its bulk properties. Surface modification of polymer films using DBD are usually conducted to improve adhesion or hydrophilicity properties, as DBD exited high-energy electrons produce ions and reactive species that interact with film surface to modify the surface characteristics. Electrical properties such as surface flashover voltage and surface resistivity can also be improved by non-thermal plasma modification [26-28].

In this section, we will study lifetime improvement and its mechanism under repetitive impulse voltages, by considering the influence of trap energy distribution on carrier transportation and charge dissipation with voltage reversion. Thus, isothermal surface potential decay (ISPD) [29, 30] technique was conducted to study the charge transport characteristics of PI films before and after surface modification, revealing the energy distribution of both electron-type and holetype traps [31].

\subsubsection{Properties of plasma-modified polyimide films}

Polyimide films were put between the air gap of DBD for surface modification, with different treating time set as $0,10,20,30,60$, and $90 \mathrm{~s}$. The DBD was excited by non-thermal plasma generator in atmospheric air, as shown in Figure 10.

The surface of the polyimide films was etched during plasma treatment. As shown in Figure 11, embossing, micro-voids, and obvious etched channels gradually appear on film surface, with increasing treating time. Based on the energy disperse spectroscopy (EDS) analysis results shown in Table 2, it is indicated that the oxygen content of plasma-treated films is higher than that of untreated film. This would be attributed to the introduction of rich-oxygen groups onto the film surface during plasma treatment.

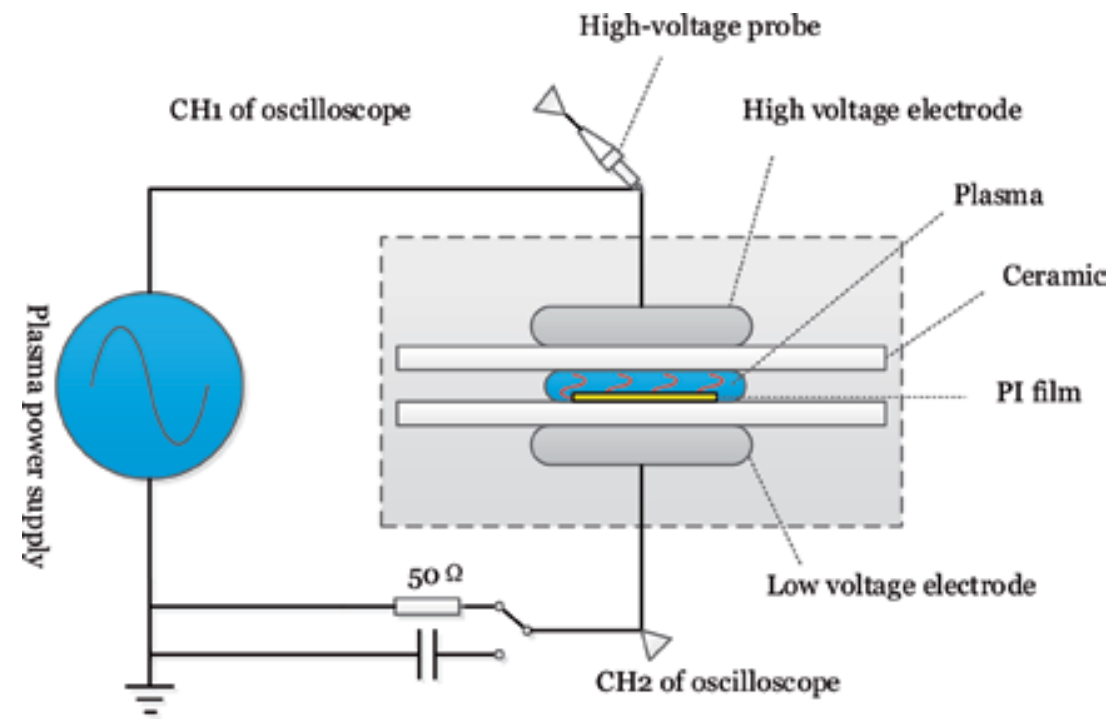

Figure 10.

Setup for dielectric barrier discharge (DBD) plasma treatment. 

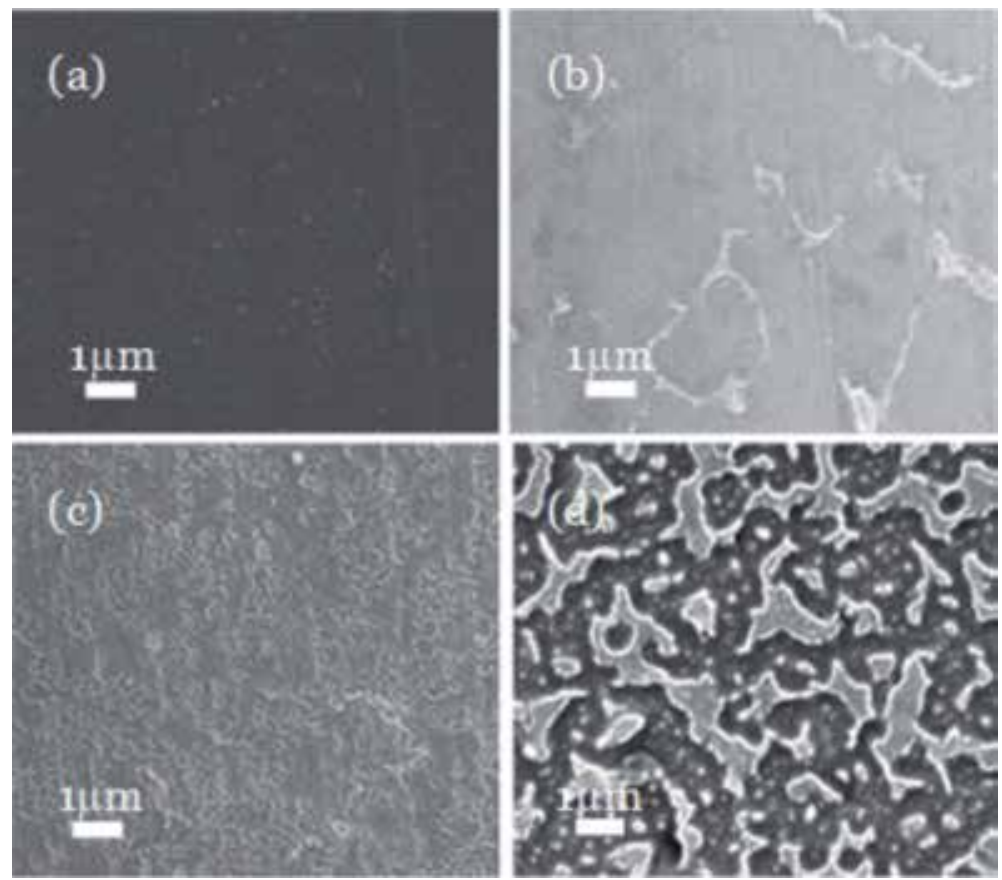

Figure 11.

SEM images of PI after different treating time. (a) PI. (b) PI-20. (c)PI-30. (d) PI-go.

\begin{tabular}{lcc}
\hline Samples & Weight percentage of C (wt \%) & Weight percentage of O (wt \%) \\
\hline PI & 21.71 & 78.29 \\
\hline PI-90 & 18.70 & 81.30 \\
\hline
\end{tabular}

Table 2.

Elemental analysis of PI films before and after plasma treatment.

Main molecular chains of PI have not been changed by plasma treatment [32, 33], while the intensities of these characteristic absorption peaks for plasma-treated films obviously decrease compared with untreated films, indicating some ring-opening reactions occurred during plasma treatment, as shown in Figure 12. Moreover, absorption bands between 3660 and $3200 \mathrm{~cm}^{-1}$, which represent $\mathrm{O}-\mathrm{H}$ stretching peaks in carboxyl acids [32,33], can be observed in plasma-treated films. Peaks appear at $1410(-\mathrm{COOH})$ and $3220(-\mathrm{NH} 2) \mathrm{cm}^{-1}$ also increase in plasma-treated films, indicating that reactive species, containing oxygen and nitrogen, have been introduced onto the film surface during plasma treatment. However, when the treating time is longer than $30 \mathrm{~s}$, the intensities of characteristic absorption peaks representing $-\mathrm{COOH}$ and $-\mathrm{NH} 2$ obviously decrease. This would be attributed to further oxidizing reactions during plasma treatment, as working gas is air. Thus, appropriate treating time is an important factor to introduce reactive groups effectively.

Lifetime under repetitive impulse voltage for plasma-treated films can be improved when the plasma treating time is less than $20 \mathrm{~s}$, but it decreases with increasing treating time, when the treating time is longer than $20 \mathrm{~s}$, as shown in Figure 13. When the treating time is 20 s, the lifetime reaches the maximum value of $23.5 \mathrm{~min}$, which is $16.9 \%$ higher than that of untreated samples. When the treating time exceeds $30 \mathrm{~s}$, the lifetime decreases sharply, by reaching the values even shorter than that of untreated samples. 


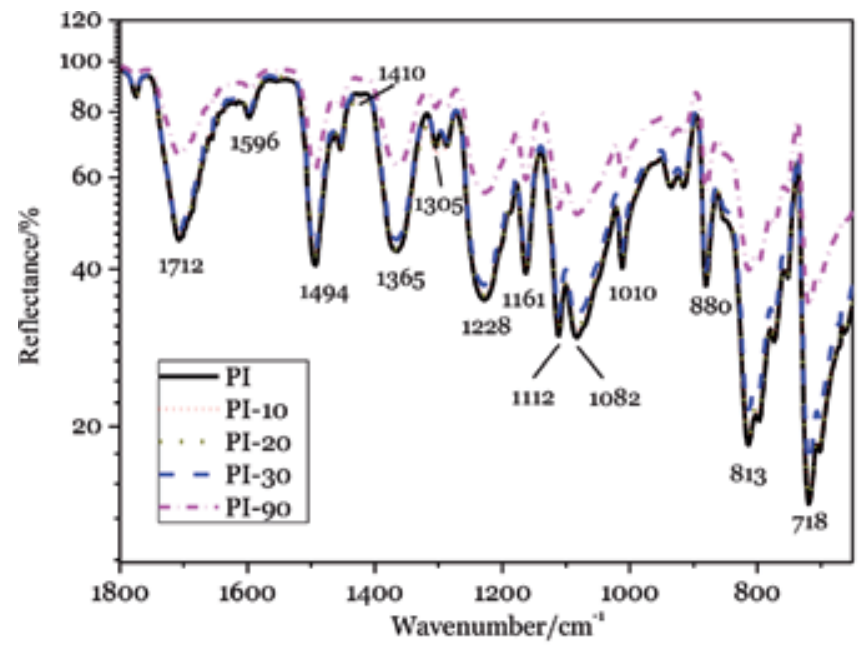

(a)

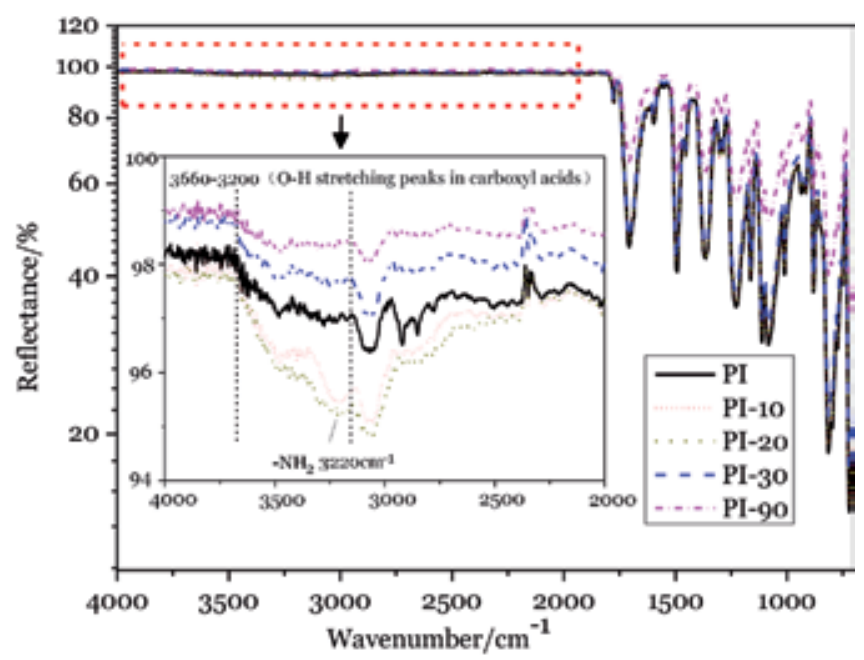

(b)

Figure 12.

FTIR spectra of PI films under different plasma treating time. (a) $1800-700 \mathrm{~cm}^{-1}$. (b) $4000-700 \mathrm{~cm}^{-1}$.

\subsubsection{Modification mechanism}

The energy levels of both electron-type and hole-type traps are lower in plasmatreated films than that in untreated ones, as shown in Figure 14. Apparent decrease of trap energies takes place when the treating time is shorter than $20 \mathrm{~s}$. However, the trap energy levels increase when the treating time exceeds $20 \mathrm{~s}$, but they are still lower than that of untreated samples. For each curve, two peaks can be observed, which represent the distribution of shallow and deep traps, respectively.

Shallow trap energy level for PI-20 is the lowest compared with the others, corresponding to the longest lifetime repetitive impulse voltages. Trap energy level dominates charge transfer characteristics rather than trap density. With lower shallow trap energy level, carriers are easier to transfer through the sample, causing less charge accumulation. For deeper traps, more energy is needed to detrap the carriers, which means more charges are trapped in samples. Only the near surface region with the depth about $\sim 90 \mathrm{~nm}$ of the material has been modified by reactive 
Failure Mechanism and Property Modification of Insulation Material Used in Inverter-Fed Motors DOI: http://dx.doi.org/10.5772/intechopen.89698

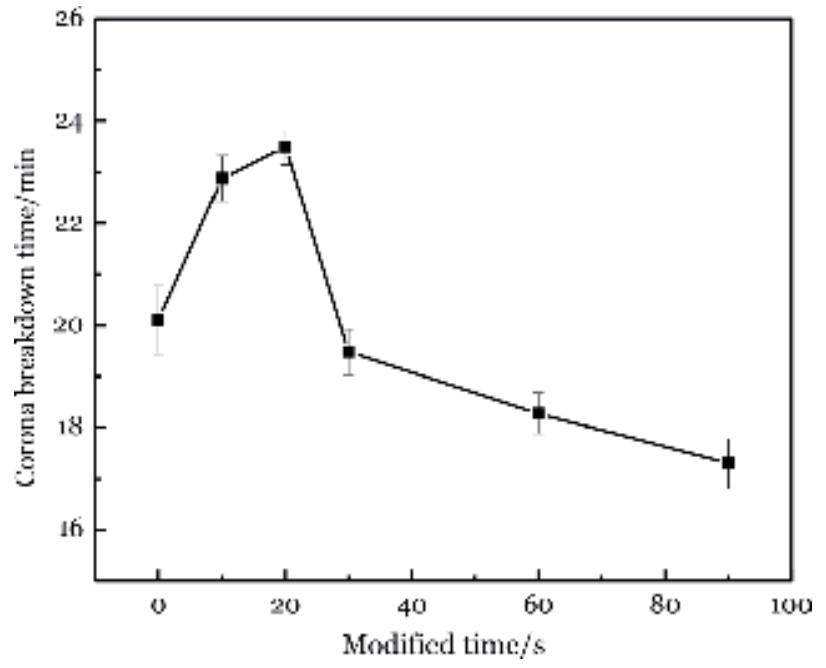

Figure 13.

Lifetime under repetitive impulse voltage with different plasma treating time.

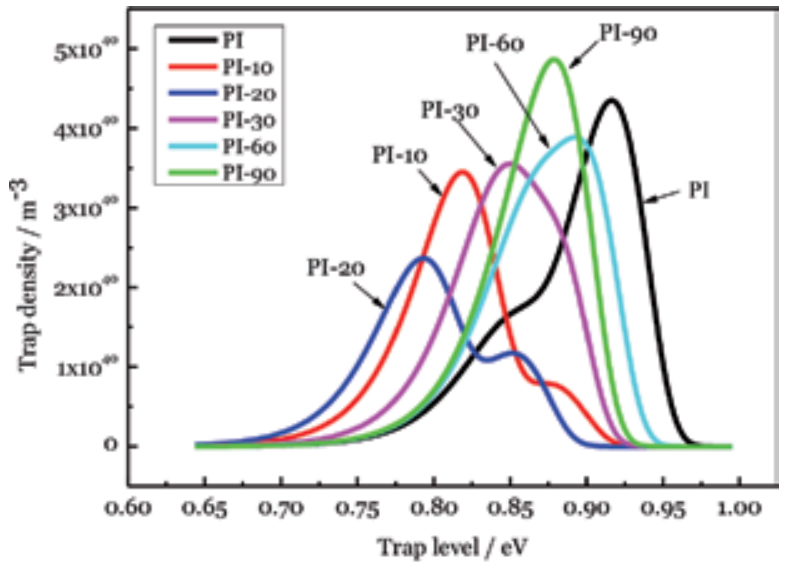

(a)

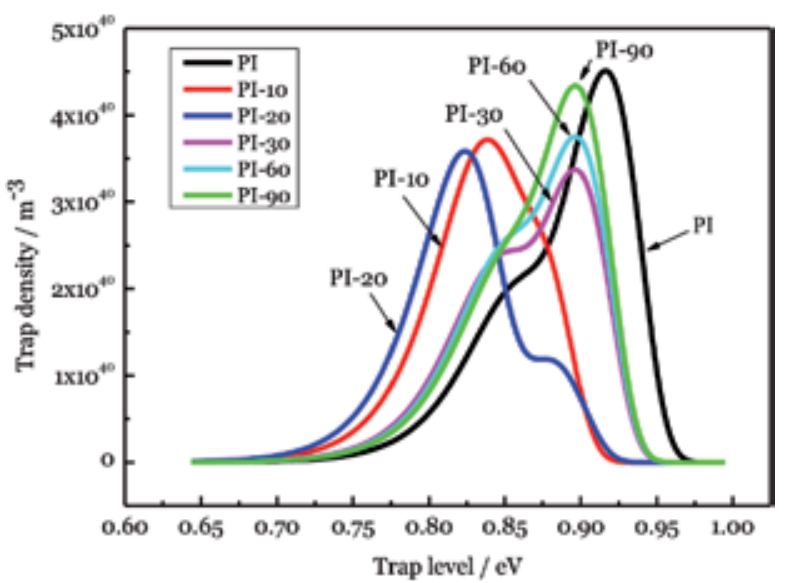

(b)

Figure 14 .

Trap energy distribution of PI films under different treating time. (a) Energy distribution of hole-type traps. (b) Energy distribution of electron-type traps. 
groups during plasma treatment [34]. Thus, the trap distribution modification mainly occurs in the near surface region, by introducing groups contained oxygen and nitrogen. Corresponding charge transportation and accumulation characteristics modification also takes place in this near surface region. When it comes to the repetitive impulse voltages, the trapped surface charges will be superposed to the applied voltage as polarity reverses, and local field would be further enhanced [23]. In this case, lifetime will be shortened due to intensive PD activity. For plasmatreated films, with lower energies of both shallow and deep traps, charge dissipation would be enhanced and the density of trapped charges would be reduced, leading to the local electric filed mitigation, resulting in suppressed PD intensity and corresponding longer lifetime.

However, when the treating time is too long, plasma-introduced oxygen and nitrogen rich groups would be broken by further oxidizing reactions, so less effective active groups have been introduced through plasma treatment. Furthermore, the degradation of PI films begins to occur, which means large molecular chains of PI began to break. It is more likely to form low-density region on the film surface. Although the charge dissipation is enhanced by the effect of shallower traps and corresponding PD activity might be weakened, this low-density region still cannot withstand the weakened PD intensity, and cleavages of the molecular chain will take place subsequently. Finally, the dielectric breakdown will occur in a short time.

\section{Conclusion}

The insulation system of inverter-fed motors is subjected to repetitive impulse voltages which are generated by pulse width modulation (PWM) converters. The insulation materials are expected to withstand partial discharge (PD) activity during service. Under repetitive impulse voltages, most of PD pulses appear next to the rising edge and the falling edge of applied voltage but rarely present during the flat region of repetitive square impulse voltages, which is quite different from the PD characteristics under power frequency. The phase distribution appears widening tendency with the increase of aging time. Especially for the phase near $20^{\circ}$, the PD number and PD quantity increase obviously.

As turn-to-turn insulation of inverter-fed traction motors, the dielectric properties of polyimide need to be improved to meet the requirement for rapid development of industry application, especially for PD-dependent lifetime under repetitive impulse voltages. Lifetime under repetitive impulse voltages can be improved by synthesizing polyimide/inorganic nanocomposites and plasma treatment.

For polyimide nanocomposites, during degradation process, inorganic particles would leave on the film surface of polyimide due to higher melting point, acting as obstacles in the erosion path of polyimide matrix. New chemistry bonds would be generated in the interfacial regions of polyimide nanocomposites, which is the lowest bonding energy. These weak bonds would be destroyed at the first stage of aging, reducing dissociations of other chemical bonds of polyimide molecules. With improved surface conductivity, charge dissipation of polyimide nanocomposites mitigates the electric field distortion, resulting in higher PDIV. As shallower traps are introduced in nanocomposites, it is less possible for charge recombination in polyimide nanocomposites, leading to lower released energy, which is beneficial to reduce the degradation of polymer.

For plasma-treated films, lower shallow trap energy level is beneficial to charge transfer; with lower deep trap energy level, less charge accumulation takes place. Trap energy level dominates the charge transportation and accumulation characteristics rather than trap density. Active groups contained oxygen and nitrogen, which 
are introduced during plasma treatment, are responsible for trap energy distribution modification of PI films. Under repetitive impulse voltage, faster surface potential decay rate and less charge accumulation can facilitate charge dissipation and local electric field mitigation, resulting in weakened PD intensity and a longer lifetime. Appropriate treating time is an important factor to introduce reactive groups and prolong the lifetime.

\section{Acknowledgements}

This work was financially supported by the National Natural Science Foundation of China (NSFC) under grant no. 51837009 and the State Key Laboratory of Electrical Insulation and Power Equipment (EIPE18212).

\section{Author details}

Yan Yang* and Guangning Wu

School of Electrical Engineering, Southwest JiaotongUniversity, China

*Address all correspondence to: yangyanyy@swjtu.edu.cn

\section{IntechOpen}

(C) 2019 The Author(s). Licensee IntechOpen. This chapter is distributed under the terms of the Creative Commons Attribution License (http://creativecommons.org/licenses/ by/3.0), which permits unrestricted use, distribution, and reproduction in any medium, provided the original work is properly cited. (cc) BY 


\section{References}

[1] Zhou L, Wu G, Gao B, et al. Study on charge transport mechanism and space charge characteristics of polyimide films. IEEE Transactions on Dielectrics and Electrical Insulation. 2009;16(4):1143-1149

[2] Hayakawa N. Partial discharge characteristics of inverter-fed motor coil samples under ac and surge voltage conditions. IEEE Electrical Insulation Magazine. 2005;21(1):5-10

[3] Kandev N, Castelan P, Lebey T, et al. Testing of low-voltage motor turn insulation intended for pulsewidth modulated applications. IEEE Transactions on Dielectrics and Electrical Insulation. 2000;7(6):783-789

[4] Yin W. Failure mechanism of winding insulations in inverter-fed motors. IEEE Electrical Insulation Magazine. 1997;13(6):18-23

[5] Luo Y, Wu G, Liu J, et al. PD characteristics and microscopic analysis of polyimide film used as turn insulation in inverter-fed motor. IEEE Transactions on Dielectrics and Electrical Insulation. 2014;21(5):2237-2244

[6] Kaufhold M, Aninger H, Berth $\mathrm{M}$, et al. Electrical stress and failure mechanism of the winding insulation in PWM-inverter-fed low-voltage induction motors. IEEE Transactions on Industrial Electronics. 2000;47(2):396-402

[7] Akram S, Wu G, Gao GQ, et al. Cavity and interface effect of PI-film on charge accumulation and PD activity under bipolar pulse voltage. Journal of Electrical Engineering and Technology. 2015;10(5):2089-2098

[8] Nehete K, Sharma RA, Chaudhari L, et al. Study of erosion resistance and mechanical properties of unsaturated polyester based nano-composites. IEEE Transactions on Dielectrics and Electrical Insulation. 2012;19(2):373-382

[9] Li Z, Okamoto K, Ohki Y, et al. Effects of nano-filler addition on partial discharge resistance and dielectric breakdown strength of micro-Al2O3/ epoxy composite. IEEE Transactions on Dielectrics and Electrical Insulation. 2010;17(3):653-661

[10] Tanaka T, Ohki Y, Ochi M, et al. Enhanced partial discharge resistance of epoxy/clay nanocomposite prepared by newly developed organic modification and solubilization methods. IEEE Transactions on Dielectrics and Electrical Insulation. 2008;15(1):81-89

[11] Maity P, Basu S, Parameswaran V, et al. Degradation of polymer dielectrics with nanometric metal-oxide fillers due to surface discharges. IEEE Transactions on Dielectrics and Electrical Insulation. 2008;15(1):52-62

[12] Kwon OJ, Tang S, Myung SW, et al. Surface characteristics of polypropylene film treated by an atmospheric pressure plasma. Surface and Coatings Technology. 2005;192(1):1-10

[13] Park WJ, Yoon SG, Jung WS, et al. Effect of dielectric barrier discharge on surface modification characteristics of polyimide film. Surface \& Coatings Technology. 2007;201(9):5017-5020

[14] Kostov KG, Nishime TMC, Hein LRO, et al. Study of polypropylene surface modification by air dielectric barrier discharge operated at two different frequencies. Surface and Coatings Technology. 2013;234:60-66

[15] Persson E. Transient effects in application of PWM inverters to induction motors. IEEE Transactions on Industry Applications. 2002;28(5):1095-1101 
[16] Stone GC, Van Heeswijk RG, Bartnikas R. Investigation of the effect of repetitive voltage surges on epoxy insulation. IEEE Transactions on Energy Conversion. 1992;7(4):754-760

[17] Wheeler JCG. Effects of converter pulses on the electrical insulation in low and medium voltage motors. IEEE Electrical Insulation Magazine. 2005;21(2):22-29

[18] Wang P, Wu G, et al. Study of partial discharge characteristics at repetitive square voltages based on UHF method. Science China Technological Sciences. 2013;56(1):262-270

[19] Morshuis PHF. Degradation of solid dielectrics due to internal partial discharge: Some thoughts on Progress made and where to go now. IEEE Transactions on Dielectrics and Electrical Insulation. 2005;12(5):905-913

[20] Mayoux C. Degradation of insulating materials under electrical stress. IEEE Transactions on Dielectrics and Electrical Insulation. 2000;7(5):590-601

[21] Zhu Y, Otsubo M, Honda C. Degradation of polymeric materials exposed to corona discharges. Polymer Testing. 2006;25(3):313-317

[22] Luo Y, Wu G, Liu J, et al. Investigation of temperature effects on voltage endurance for polyimide/A12O3 nanodielectrics. IEEE Transactions on Dielectrics and Electrical Insulation. 2014;21(4):1824-1834

[23] Zhong X, Wu G, Yang Y, et al. Effects of nanoparticles on reducing partial discharge induced degradation of polyimide/Al2O3 nanocomposites. IEEE Transactions on Dielectrics and Electrical Insulation.

2018;25(2):594-602

[24] Liufu D, Wang X, Tu D, et al. High-field induced electrical aging in polypropylene films. Journal of Applied Physics. 1998;83(4):2209-2214

[25] Shao T, Zhang C, Long K, et al. Surface modification of polyimide films using unipolar nanosecond-pulse DBD in atmospheric air. Applied Surface Science. 2010;256(12):3888-3894

[26] Shao T, Yang W, Zhang C, et al. Enhanced surface flashover strength in vacuum of polymethylmethacrylate by surface modification using atmospheric-pressure dielectric barrier discharge. Applied Physics Letters. 2014;105(7):082903-082264

[27] Shao T, Liu F, Hai B, et al. Surface modification of epoxy using an atmospheric pressure dielectric barrier discharge to accelerate surface charge dissipation. IEEE Transactions on Dielectrics and Electrical Insulation. 2017;24(3):1557-1565

[28] Zhao W, Xu R, Ren C, et al. Ionimplantation modification of surface flashover properties in vacuum of polytetrafluoroethylene. IEEE Transactions on Plasma Science. 2018:1-7

[29] Li J, Zhou F, Min D, et al. The energy distribution of trapped charges in polymers based on isothermal surface potential decay model. IEEE Transactions on Dielectrics and Electrical Insulation. 2015;22(3):1723-1732

[30] Min D, Cho M, Khan AR, et al. Surface and volume charge transport properties of polyimide revealed by surface potential decay with genetic algorithm. IEEE Transactions on Dielectrics and Electrical Insulation. 2012;19(2):600-608

[31] Han Y, Li S, Min D. Trap energy distribution in polymeric insulating materials through surface potential decay method. IEEE Transactions on Dielectrics and Electrical Insulation. 2018;25(2):639-648 
[32] Park SJ, Lee HY. Effect of atmospheric-pressure plasma on adhesion characteristics of polyimide film. Journal of Colloid and Interface Science. 2005;285(1):267-272

[33] Yang Y, Yin D, Xiong R, et al. Ftir and dielectric studies of electrical aging in polyimide under $\mathrm{AC}$ voltage. IEEE Transactions on Dielectrics and Electrical Insulation. 2012;19(2):574-581

[34] Park B, Kim J, Cho M, et al. Ballistic-mode plasma-based ion implantation for surface-resistivity modification of polyimide film. IEEE Transactions on Plasma Science. 2012;40(6):1749-1752 


\title{
Dielectric Analysis Model for Measurement of Soil Moisture Water Content Using Electrical Capacitance Volume Tomography
}

\author{
Mukhlisin Muhammad and Saputra Almushfi
}

\begin{abstract}
Electromagnetic methods have been widely used in the measurement of the water content of the soil. These methods utilize the permittivity as electrical properties of the soil, to determine the moisture content of the soil. Since the measurements are carried out indirectly, a calibration between permittivity and the water content of the soil is needed. Generally, the calibration method is generated by using an empirical and mixing model. This study presents a proposed model of calibration by using a normalization approach to calibrate the value of the permittivity of the water content of the soil. Then the model was applied using electrical capacitance volume tomography (ECVT) to image soil water content during infiltration of water in a soil column. Granular and silty sand were used as soil material in the experiments. The result showed that the model for measuring moisture water content can be seen in each layer during soil water infiltration in the soil column.
\end{abstract}

Keywords: dielectric analysis model, soil moisture water content, ECVT

\section{Introduction}

Measurement of soil moisture water content has become an important part of the analysis of various fields of study, especially those involving irrigation in agriculture, forestry, hydrology, and land activity. For example, in agriculture study, it is required to determine water source and ensure the quality of crop [1]. Moreover, soil moisture water content is useful to analyze soil water contamination by observing changes in water content during the addition of substance [2]. Soil water content also plays an important role in slope stability analysis [3-6].

Various techniques in the measurement of contamination and water content of soil have been discussed in literatures (e.g., [7-12]). Based on previous studies, soil water content measurement techniques are widely used as an electromagnetic method [13] such as time domain reflectometry [14], ground-penetrating radar (GPR) [8], and electrical capacitance [15]. These all methods measure the value of relative permittivity of soil to find soil moisture water content.

Tomography is a promising technique for measurement of water content in soil, especially for capacitance-based tomography. It is because this technique is not only capable of measuring water content in the soil but also is capable of imaging the 
distribution of water in the soil. Tomography technique is also preferred because it is nondestructive and noninvasive. As a tomography technique, ECVT is a system used to view enclosed objects by measuring changes in capacitance and then compute relative permittivity distribution to create three-dimensional images in real time [16]. The shape of geometry sensor on ECVT is not confined to one form; it can be in the form of arbitrary shape of geometries [17]. This possibility gives an extra advantage of ECVT in measuring soil water content.

The previous study has successfully monitored the propagation of distribution of water in soil column [18]. In this study, the equation of normalized volumetric water content from ECVT method is proposed and compared with other equations. The proposed model is then used to analyze the volumetric water content during soil water infiltration in a vessel.

\section{ECVT principle}

The basic measurement of ECVT is derived from Poisson's equation:

$$
\nabla \cdot(\varepsilon(x, y, z) \nabla \varphi(x, y, z)=-\rho(x, y, z))
$$

where $\varepsilon$ is relative permittivity distribution, $\phi$ is electric potential, and $\rho$ is charge distribution. From Eq. (1), capacitance value can be obtained by using the equation below:

$$
C=\frac{1}{\Delta V} \oint_{\Gamma} \varepsilon(x, y, z) \nabla \varphi(x, y, z) \cdot \hat{n} d l
$$

where $\Delta V$ is potential difference and $C$ is capacitance. By using matrix expression, Eq. (2) can be written like the following equation:

$$
\mathrm{C}=\mathrm{S} \mathbf{G}
$$

where $\mathbf{C}$ is capacitance matrix, $\mathbf{G}$ is distribution of relative permittivity matrix, and $\mathbf{S}$ is sensitivity matrix. The sensitivity matrix is generated from the sensor and geometry design and number of sensors. In matrix operation, the value of $\mathbf{G}$ can be obtained by inversing matrix $\mathbf{S}$ and multiplying it with matrix $\mathbf{C}$. For non-square matrix, matrix inversion is very difficult to solve, so the approximation could be attempted by using transpose matrix. The equation to calculate matrix $\mathbf{G}$ becomes

$$
\mathbf{G}=\mathbf{S}^{\mathbf{T}} \mathbf{C}
$$

Equations (3) and (4) are known as the forward and inverse problem, respectively. Inverse problem is used to reconstruct the capacitance measurements to become relative permittivity distribution. The simple method for reconstruction is using linear back projection (LBP) [19].

\section{Models of soil moisture water content and relative permittivity relationship}

The relationship between relative permittivity and volumetric water content has been used by previous researchers to determine the volumetric water content. Many numbers of functions have been proposed to describe the $\varepsilon-\theta$ relationship 
model across a range of soil water content. This model can be divided into two categories, which are model with one parameter and model with two or more parameters.

\subsection{Model with one parameter}

There are some models that proposed the $\varepsilon-\theta$ relationship. Topp et al. [20] introduced successfully the $\varepsilon-\theta$ relationship that is commonly used in geotechnical area. The relationship is as shown below:

$$
\varepsilon=3.03+9.3 \theta+146.0 \theta^{2}-76.7 \theta^{3}
$$

where $\varepsilon$ is the relative permittivity or dielectric constant and $\theta$ is the volumetric water content of soil.

Equation (1) is derived empirically through experiments of various of mineral soil using a time-domain reflectometer (TDR) at a frequency between $1 \mathrm{MHz}$ and $1 \mathrm{GHz}$, with an estimated error value of 0.013 [20]. In another form, Topp's equation can also be written as follows:

$$
\theta=-5.3 \times 10^{-2}+2.92 \times 10^{-2} \varepsilon-5.5 \times 10^{-4} \varepsilon^{2}+4.3 \times 10^{-6} \varepsilon^{3}
$$

In addition, Topp et al. [20] also proposed equations of $\varepsilon-\theta$ relationship for organic soil and $450 \mu \mathrm{m}$ glass beads.

$$
\begin{gathered}
\varepsilon=1.74-0.34 \theta+135 \theta^{2}-55.3 \theta^{3} \quad \text { Organic soil } \\
\varepsilon=3.57+31.7 \theta+114 \theta^{2}-68.2 \theta^{3} \quad 450-\mu \text { m glass beads }
\end{gathered}
$$

Then Roth et al. [21] proposed another empirical equation by an experiment using miniprobe TDR which has been used previously by [22]. The $\varepsilon-\theta$ relationship for mineral soil proposed by [21] is

$$
\theta=-0.0728+0.0448 \varepsilon-0.00195 \varepsilon^{2}+0.0000361 \varepsilon^{3}
$$

while the $\varepsilon-\theta$ relationship for organic soil and material is

$$
\theta=-0.0233+0.0285 \varepsilon-0.000431 \varepsilon^{2}+0.00000304 \varepsilon^{3}
$$

Calibration of these equations has a volumetric water content error value of $0.015 \mathrm{~cm}^{3} \mathrm{~cm}^{-3}$ for mineral soil and $0.035 \mathrm{~cm}^{3} \mathrm{~cm}^{-3}$ for organic soil [22].

Simple equation of $\varepsilon-\theta$ relationship was proposed by [23]. This equation resulted from the principal of dielectric mixing models and analyzed the TDR without coatings that are considered potential sources of error in measurement:

$$
\theta=0.1181 \sqrt{\varepsilon}-0.1841
$$

Schaap et al. [24] also introduce a simple equation by performing experiments of 505 measurements of organic forest floor sample by using TDR where the $\varepsilon-\theta$ relationship is

$$
\theta=0.136 \sqrt{\varepsilon}-0.119
$$

The next equation comes from [25], which is using coaxial transmission system and using soil samples with wide range of soil textures: 


$$
\theta=-0.0286+0.02435 \varepsilon-0.0003421 \varepsilon^{2}+0.00000237 \varepsilon^{3}
$$

Equations (5)-(8) are used for measurement at a frequency of $100 \mathrm{MHz}$.

[13] proposed an empirical model where permittivity measurement was measured based on capacitance. This experiment used a type of quartz sand with a range of particle sizes between 0.15 and $0.9 \mathrm{~mm}$ :

$$
\varepsilon=A\left(\frac{1}{1+(\alpha(1-\theta))^{n}}\right)^{1-\frac{1}{n}}+B
$$

where $\mathrm{A}=33, \mathrm{~B}=2, \alpha=1.5$, and $\mathrm{n}=14$.

\subsection{Model with two or more parameters}

Some relationship equations between permittivity and soil water content were also influenced by other parameters such as porosity and bulk density. By using the concept of mixing models and using data from others study [26-28], [29] proposed the following equations:

$$
\varepsilon=\theta\left(\varepsilon_{i}+\left(\varepsilon_{w}-\varepsilon_{i}\right) \frac{\theta}{\theta_{t}} \gamma\right)+(\eta-\theta) \varepsilon_{a}+(1-\eta) \varepsilon_{r}
$$

Equation (11) is used for $\theta \leq \theta_{t}$, while $\theta>\theta_{t}$ used the following equation:

$$
\varepsilon=\theta_{t}\left(\varepsilon_{i}+\left(\varepsilon_{w}-\varepsilon_{i}\right) \gamma\right)+\left(\theta-\theta_{t}\right) \varepsilon_{w}+(\eta-\theta) \varepsilon_{a}+(1-\eta) \varepsilon_{r}
$$

where $\varepsilon_{i}, \varepsilon_{w}, \varepsilon_{a}$, and $\varepsilon_{r}$ are the permittivity of ice, water, air and rock, respectively (i.e., $\varepsilon_{i}=3.2, \varepsilon_{w}=80$, and $\varepsilon_{a}=1$ ), while $\theta_{t}$ is transition moisture (0.16-0.33), $\eta$ is the porosity of soil (0.5), and $\gamma$ is the fitting parameter (0.3-0.5) [29].

In [30], the equation based on dielectric mixing model, which has been described by [31], is proposed. Experiments carried out by measuring a wide range of soil types using TDR with the error value of soil water content is not more than $0.013 \mathrm{~cm}^{3} \mathrm{~cm}^{-3}$ [30], with forms of the equation below:

$$
\begin{array}{cc}
\theta=\frac{\varepsilon^{\gamma}-(1-\eta) \varepsilon_{s}^{\gamma}-\eta \varepsilon_{a}^{\gamma}}{\varepsilon_{w}^{\gamma}-\varepsilon_{a}^{\gamma}} & ; \gamma=-1 \\
\theta=\frac{\varepsilon^{\gamma}-(1-\eta) \varepsilon_{s}^{\gamma}-\eta \varepsilon_{a}^{\gamma}}{\varepsilon_{w}^{\gamma}-\varepsilon_{a}^{\gamma}} & ; \gamma=1
\end{array}
$$

where $\gamma=-1$ for three phases in series and $\gamma=1$ for three phases in parallel.

Another model is proposed by [32]. They conducted the experiments by using TDR and 62 kinds of soil sample that consist of mineral soils, organic soil, standard pot soils, artificial peat loess and peat sand, sea and river sand, forest litter, etc. which differ in terms of texture and bulk density [32].

$$
\theta=\frac{\sqrt{\varepsilon}-3.47+6.22 \eta-3.82 \eta^{2}}{7.01+6.89 \eta-7.83 \eta^{2}}
$$

Equation (13) gives the uncertainty of soil water content value of 0.03 .

Gardner et al. [15] used capacitance measurement methods to obtain soil water content with the soil dry bulk density values ranging between 1.08 and 1.49 , then 
using multiple linear regression analysis to best fit the measurement data, resulting in the following equation:

$$
\theta=\frac{\sqrt{\varepsilon}+1.208-2.454 \rho}{9.93}
$$

where $\rho$ is dry bulk density.

Robinson et al. [14] give the equation used for coarse-textured, layered soils by using TDR and coarse-grained, glass bead, and quartz grains:

$$
\theta=\eta\left(\frac{\sqrt{\varepsilon}-\sqrt{\varepsilon_{d r y}}}{\sqrt{\varepsilon_{s a t}}-\sqrt{\varepsilon_{d r y}}}\right)
$$

where $\varepsilon_{d r y}$ and $\varepsilon_{\text {sat }}$ are the permittivity measured at oven dry soil and saturation soil (Table 1).

\subsection{Comparison using existing data}

Data from previous research (e.g., [20, 21, 25-27, 29, 31-35]) are used to compare the patterns of the equations which are discussed in this study. This data consists of various soil types with different properties.

Figure 1 shows several curves representing Eqs. (5)-(14). All equations look occupied by all the available data. However, each equation appears to have certain characteristics to the data. Eqs. (8) and (12) only cover the boundary area of the data, while the other equations lie mostly in the central part of the data. These would seem to depend on the properties of the soil types used. One of the characteristics analyzed in this study is the porosity of the soil.

Figure 2 shows the influence of porosity $(\eta=0.3$ to $\eta=0.7)$, on the suitability of the equations (Eqs. (15), (16), (18) and (19)-(21)) with data, and also displayed some of the data with a value of porosity $(0.33,0.44$, and 0.62$)$ in order to see the effect of porosity on predictions of water content of the equation. From the image it can be seen that the different porosity values of the data will result in different patterns.

In Figure 2a, the equations already have the same pattern with the data, but a change of porosity in the equation does not give a significant effect on the pattern of the line, so it is only fit in certain small area of data though with different porosity.

Figure $\mathbf{2 b}$ shows that the equation is such as the linear equation that has not affected on the changes in porosity. These equations also appear not to follow the pattern of distribution of data. The same thing happened in Figure $2 \mathrm{c}$ with a shift in values on the $\mathrm{x}$-axis which is more to the left.

An overestimated result is produced in Figure 2e, where the equation is not able to cover all areas of data; whereas in Figure $\mathbf{2 f}$, it has been seen covering almost all areas except the data on water content values smaller than 0.2 , but this equation has not been able to adjust to the data that have a value of porosity. The only equation that gives the better approach is Figure 2 d. This equation produces a pattern in accordance with the existing data. The equation is also seen fit to data that has a value of porosity.

In this study the relative permittivity was analyzed by the ECVT system generated in the form of normalization. Normalized volumetric water content can be defined as

$$
\Theta=\frac{\theta-\theta_{r}}{\theta_{s}-\theta_{r}}
$$




\begin{tabular}{|c|c|c|c|c|c|c|}
\hline \multirow[t]{2}{*}{ Eq. } & \multirow[t]{2}{*}{ Source } & \multirow[t]{2}{*}{ Experimental method } & \multirow[t]{2}{*}{ Soil type } & \multicolumn{3}{|c|}{ Properties of soil } \\
\hline & & & & $\begin{array}{l}\text { Porosity } \\
\left(\mathrm{cm}^{3} \mathbf{c m}^{-3}\right)\end{array}$ & $\begin{array}{l}\text { Bulk density } \\
\left(\mathrm{g} \mathrm{cm}^{-3}\right)\end{array}$ & $\begin{array}{l}\text { Particle } \\
\text { density } \\
\left(\mathrm{g} \mathrm{cm}^{-3}\right)\end{array}$ \\
\hline \multicolumn{7}{|c|}{ Model with one parameter } \\
\hline (5) & \multirow[t]{4}{*}[20]{} & \multirow{4}{*}{$\begin{array}{l}\varepsilon: \text { using TDR Tektronix } \\
\text { 7S12 model to perform } 18 \\
\text { experiments with } \\
\text { different treatments } \\
\theta: \text { using gravimetric } \\
\text { technique }\end{array}$} & - Mineral soil & \multirow{2}{*}{-} & \multirow{2}{*}{$\begin{array}{l}\cdot 1.04-1.44 \\
\cdot \quad 0.422 \\
\cdot 1.08 \\
\cdot 1.49-1.61\end{array}$} & \multirow[t]{2}{*}{-} \\
\hline (6) & & & $\begin{array}{l}\text { - Organic } \\
\text { soil } \\
\text { - Vermiculite } \\
\text { - Glass beads } \\
\end{array}$ & & & \\
\hline (7) & & & Organic soil & - & 0.422 & - \\
\hline (8) & & & $\begin{array}{l}450 \mu \mathrm{m} \text { glass } \\
\text { beads }\end{array}$ & - & $1.60-1.61$ & - \\
\hline (9) & \multirow[t]{2}{*}[21]{} & \multirow{2}{*}{$\begin{array}{l}\varepsilon: \text { TDR miniprobe } 250 \text { ps } \\
\text { rise-time needle pulse } \\
\theta: \text { gravimetric technique }\end{array}$} & $\begin{array}{l}9 \text { mineral } \\
\text { soils }\end{array}$ & $\begin{array}{l}0.418- \\
0.482\end{array}$ & $1.26-1.55$ & $2.28-2.67$ \\
\hline (10) & & & 7 organic soils & $0.527-0.785$ & $0.2-0.77$ & $\begin{array}{l}0.70- \\
1.63\end{array}$ \\
\hline (11) & {$[23]$} & $\begin{array}{l}\text { Using model of inverse } \\
\text { averaging for TDR } \\
\text { method by analyzing the } \\
\text { mixing model }\end{array}$ & - & - & - & - \\
\hline (12) & [24] & $\begin{array}{l}\varepsilon: \text { TDR Tektronix } 1502 B \\
\theta: \text { gravimetric technique }\end{array}$ & $\begin{array}{l}25 \text { samples of } \\
\text { forest floors }\end{array}$ & - & $0.086-0.263$ & 1.3 \\
\hline (13) & {$[25]$} & $\begin{array}{l}\text { Coaxial transmission/ } \\
\text { reflection apparatus } \\
\text { controlled by a } \\
\text { Hewlett-Packard } 8510 \mathrm{C} \\
\text { Vector Network Analyzer } \\
\text { system } 45 \mathrm{MHz} \text { to } \\
26.5 \mathrm{GHz}\end{array}$ & - & - & - & - \\
\hline (14) & {$[13]$} & $\begin{array}{l}\varepsilon: \text { based on capacitance } \\
\text { measurement } \\
\theta: \text { gravimetric technique }\end{array}$ & Quartz sand & - & - & - \\
\hline \multicolumn{7}{|c|}{ Model with two or more parameters } \\
\hline$(15)$ & \multirow[t]{2}{*}[29]{} & \multirow{2}{*}{$\begin{array}{l}\text { Modeling by using } \\
\text { data from other studies } \\
{[26-28]}\end{array}$} & \multirow{2}{*}{$\begin{array}{l}22 \text { different } \\
\text { samples }\end{array}$} & \multirow[t]{2}{*}{$0.4-0.6$} & \multirow[t]{2}{*}{$1.1-1.7$} & \multirow[t]{2}{*}{$2.6-2.75$} \\
\hline$(16)$ & & & & & & \\
\hline (17) & \multirow[t]{2}{*}{ [30] } & \multirow[t]{2}{*}{ TDR } & \multirow{2}{*}{$\begin{array}{l}\text { From } 11 \\
\text { different field } \\
\text { sites }\end{array}$} & & & \\
\hline$(18)$ & & & & & & \\
\hline (19) & {$[32]$} & TDR CAMI & $\begin{array}{l}62 \text { kinds of } \\
\text { soil samples }\end{array}$ & $0.33-0.95$ & $0.13-1.66$ & $1.06-2.7$ \\
\hline (20) & {$[15]$} & $\begin{array}{l}\text { Capacitance probe } 80- \\
150 \mathrm{MHz}\end{array}$ & 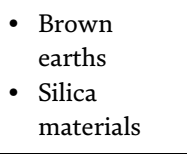 & - & $\begin{array}{l}\cdot 1.08-1.49 \\
\cdot 1.24-1.63\end{array}$ & - \\
\hline (21) & [14] & TDR Tektronix 1502B & $\begin{array}{l}\text { Coarse- } \\
\text { grained, } \\
\text { quartz grain, } \\
\text { sandy soil }\end{array}$ & - & - & - \\
\hline
\end{tabular}

Table 1.

Summary of all equations of $\varepsilon-\theta$ relationship. 
Dielectric Analysis Model for Measurement of Soil Moisture Water Content Using Electrical... DOI: http://dx.doi.org/10.5772/intechopen.89057

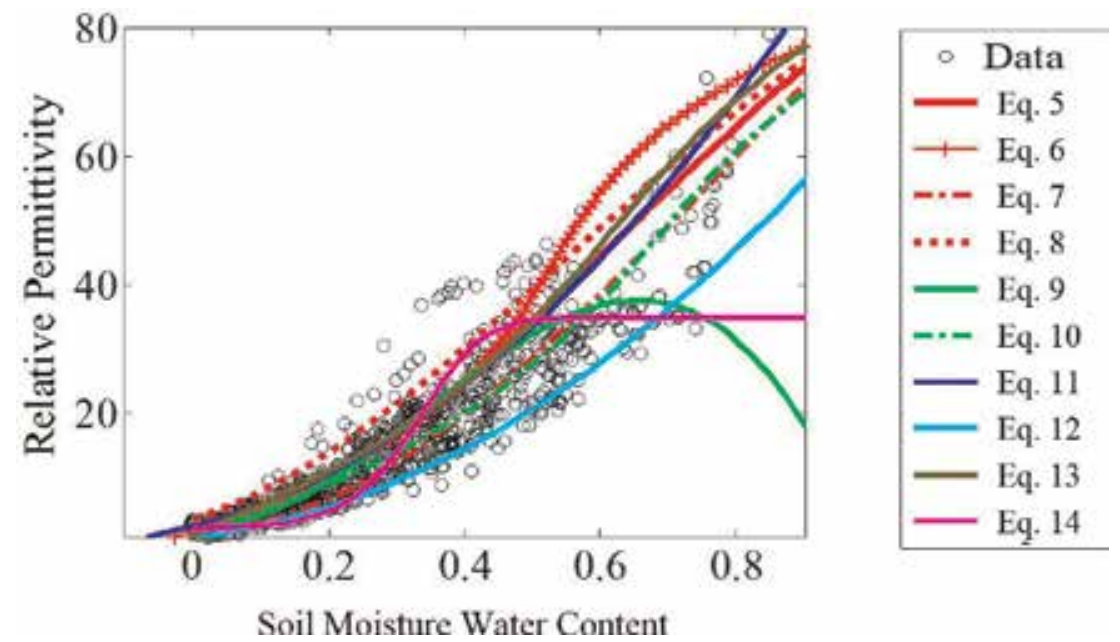

Figure 1.

Comparisons using all data for Eqs. (5)-(14).
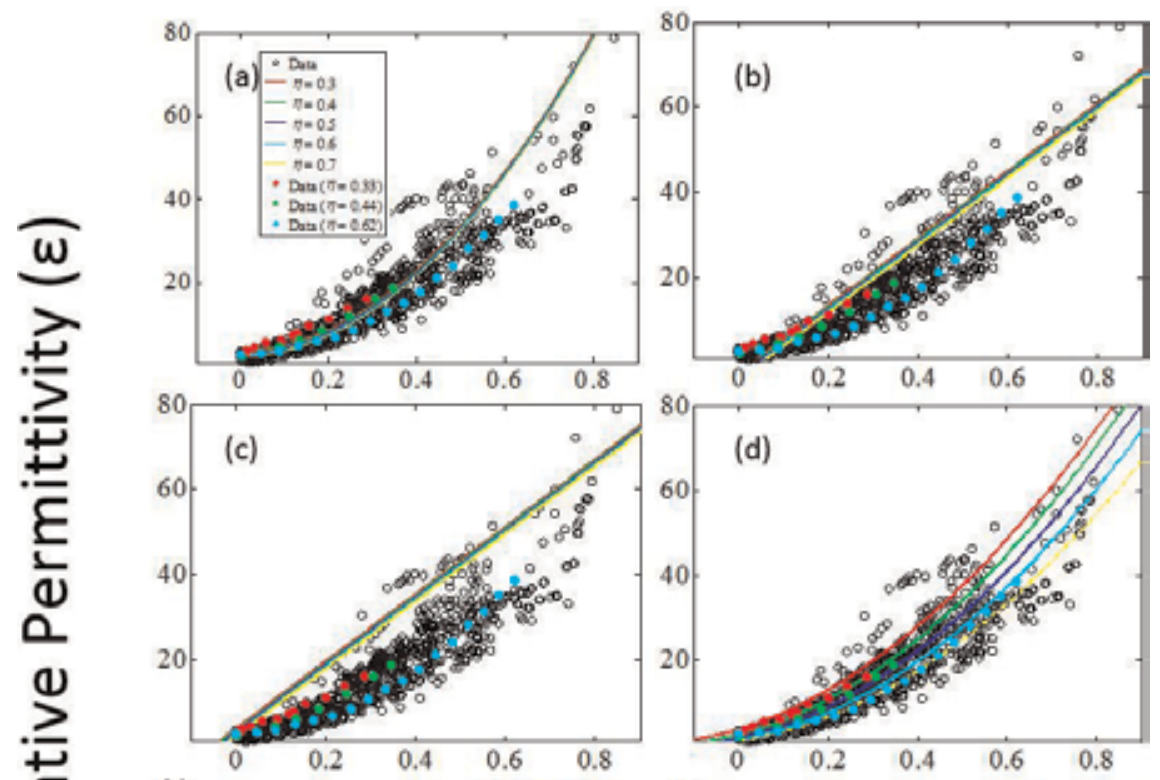

$\frac{\pi}{\mathscr{D}}$
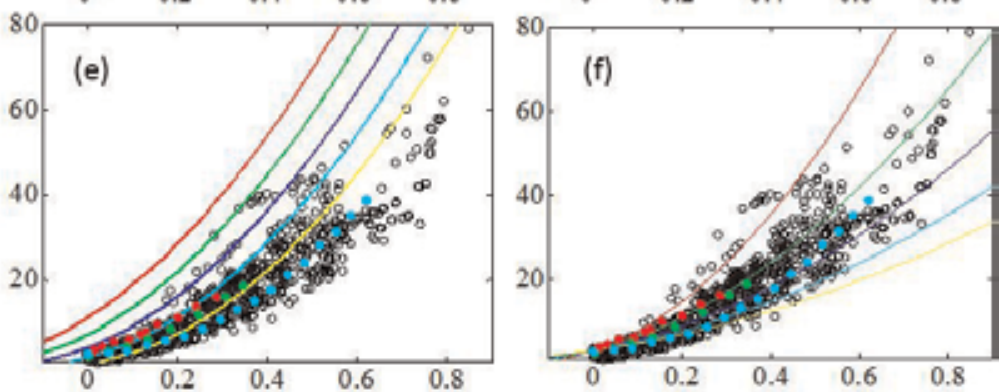

\section{Soil Moisture Water Content $(\theta)$}

Figure 2.

Comparison all equations with different porosity, by using: a) Eq. 15; b) Eq. 16; c) Eq. 18; d) Eq. 19; e) Eq. 20;f) Eq. 21. 


\begin{tabular}{lcc}
\hline Material & Relative permittivity & Elements \\
\hline Air & 1 & $\mathrm{~N}_{2}, \mathrm{O}_{2}$ \\
\hline Water & 80 & $\mathrm{H}_{2} \mathrm{O}$ \\
\hline Dry soil & $3-10$ & $\mathrm{~N}, \mathrm{P}, \mathrm{K}, \mathrm{Ca}, \mathrm{Mg}, \mathrm{S}, \mathrm{Cu}, \mathrm{Zn}, \mathrm{Fe}, \mathrm{Mn}, \mathrm{B}, \mathrm{Cl}, \mathrm{Na}, \mathrm{H}$ \\
\hline
\end{tabular}

Table 2.

Material properties.

where $\theta$ is the volumetric water content, $\theta_{r}$ is the residual volumetric water content, and $\theta_{s}$ is the saturated volumetric water content.

The normalization of relative permittivity gives privilege to define normalized volumetric water content, in this study it is assumed in three models:

$$
\begin{aligned}
\Theta & =\varepsilon_{N}^{0.5} \\
\Theta & =\varepsilon_{N} \\
\Theta & =\varepsilon_{\mathrm{N}}{ }^{2}
\end{aligned}
$$

where $\varepsilon_{N}$ is normalized permittivity which can be calculated as

$$
\varepsilon_{N}=\frac{\varepsilon-\varepsilon_{\text {air }}}{\varepsilon_{\text {water }}-\varepsilon_{\text {air }}}
$$

where $\varepsilon, \varepsilon_{\text {air }}$, and $\varepsilon_{\text {water }}$ are actual relative permittivity measurement, relative permittivity of air, and relative permittivity of water, respectively (Table 2 ).

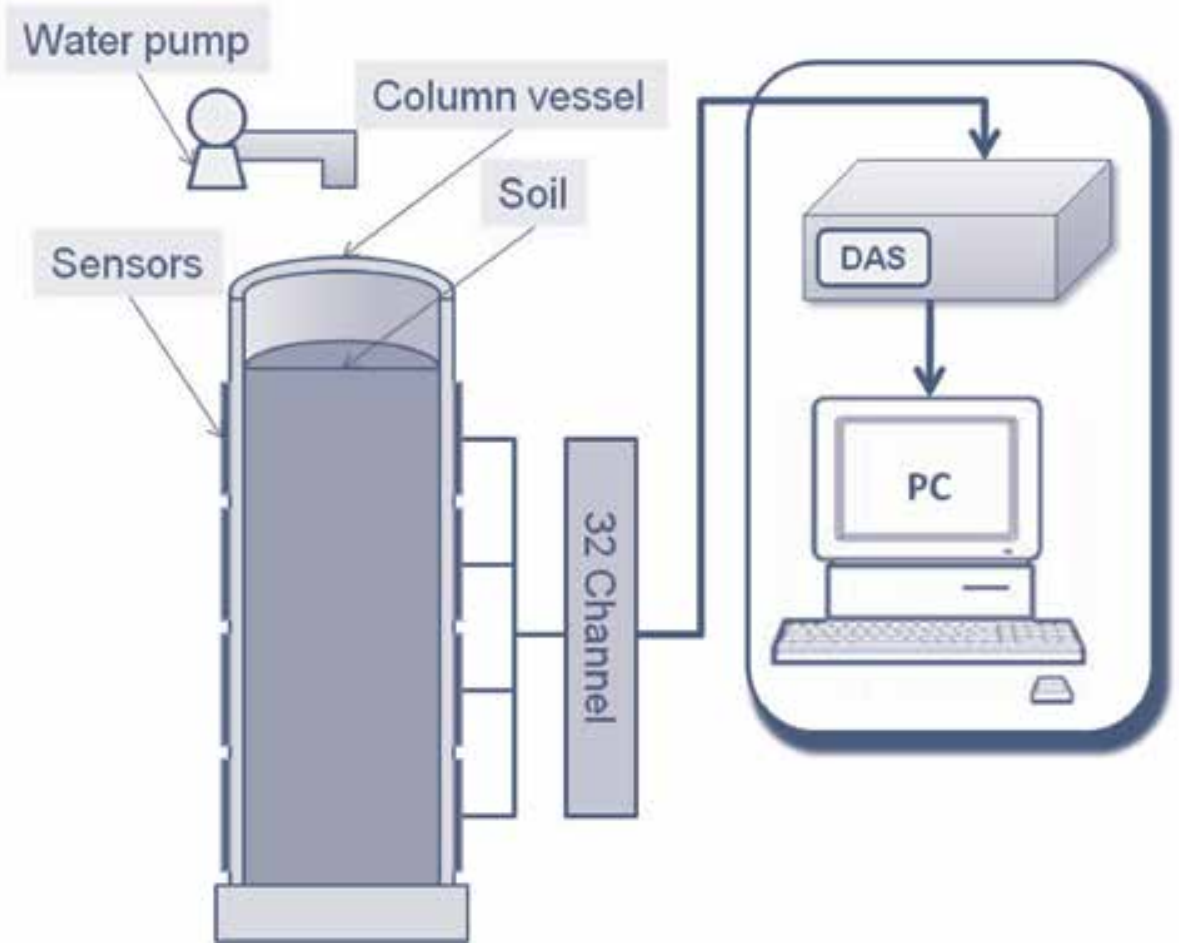

Figure 3 .

Experimental setup [15]. 


\section{Experimental setup}

ECVT system consists of three parts: (i) sensors, (ii) data acquisition, and (iii) system reconstruction and visualization as shown in Figure 3. In this experiment we used 32 channel hexagonal sensors for the first experiment and 24 channel hexagonal sensors for the second experiment, as a soil column with diameter and height 11.5 and $27 \mathrm{~cm}$, respectively. This column is divided into 32 layers with the 1st layer as the top layer and 32nd layer as the bottom layer.

In the first experiment, 31 by volume of soil in the column was supplied. The soil material that was used in this study was sand collected from the Cisadane River in Tangerang, Indonesia. The soil contained $17 \%$ fine sand and $83 \%$ medium sand with porosity of soil $41.79 \%$. The specific gravity and soil density were 2.663 and $1.55 \mathrm{~g} \mathrm{~cm}^{-3}$, respectively. In this experiment, the soil in the vessel was supplied with water flow with a discharge of $7.2 \mathrm{ml} / \mathrm{s}$ until ponded condition, and the discharge was stopped when the pond of water level was at $2 \mathrm{~cm}$ above the surface soil. During ponded condition, the data capacitances were measured iteratively and sent to the computer. The data acquisition frequency was set to one frame per second.

In the second experiment, $3 \mathrm{~kg}$ of silty sand was supplied into the column. After that, 1.4 liter of water was filled into the soil column using constant head method, in which height of the water is maintained constant by a distance of $4 \mathrm{~cm}$ from the surface of soil.

\section{Result and discussion}

Normalized volumetric water content and relative permittivity relationship of several equations is shown in Figure 4. The first model of the proposed model seems to have a similar pattern with Topp et al. [20] and Malicki et al. [32] models. By adding some constants value to the first model, this model will be quite fit with

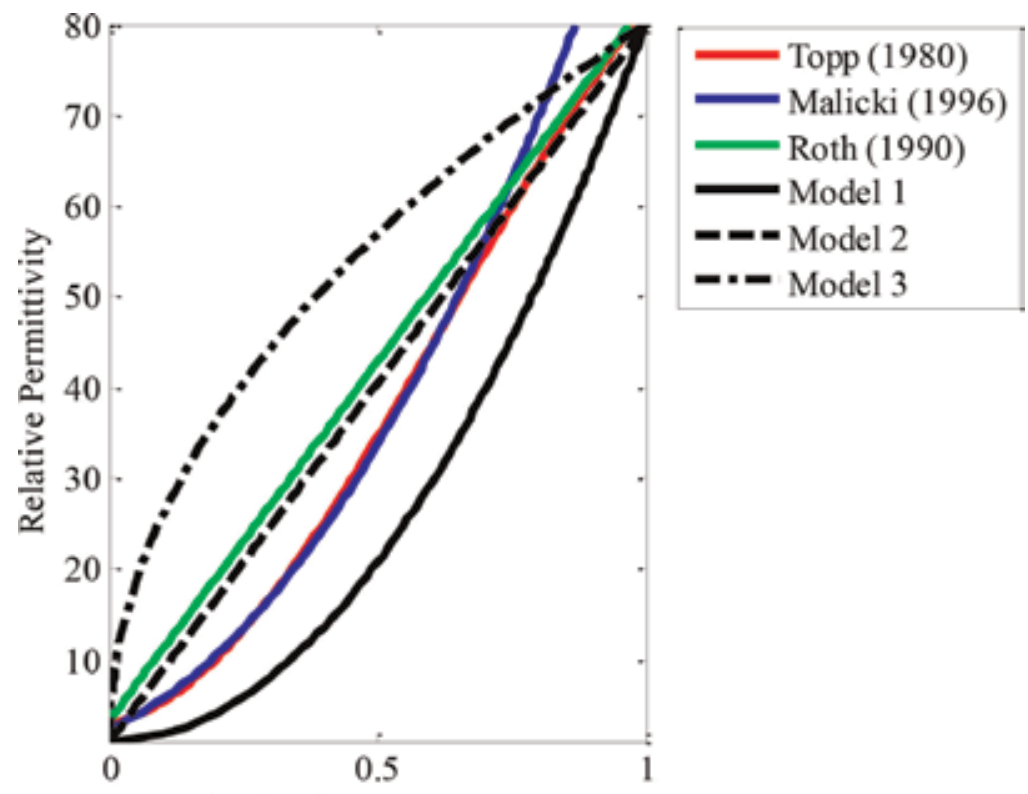

Normalized Volumetric Water Content

Figure 4.

Normalized volumetric water content and relative permittivity relationship of the three proposed models compared with established models. 


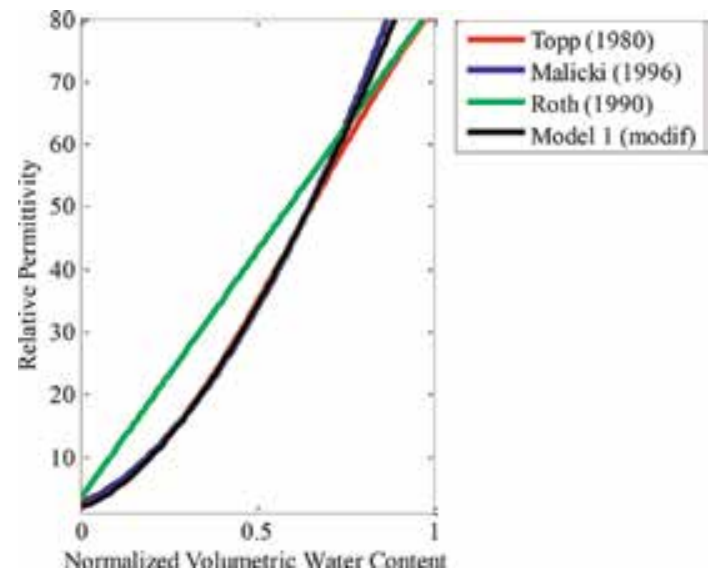

Figure 5.

Normalized volumetric water content and relative permittivity relationship of the first model modification compared with established models.
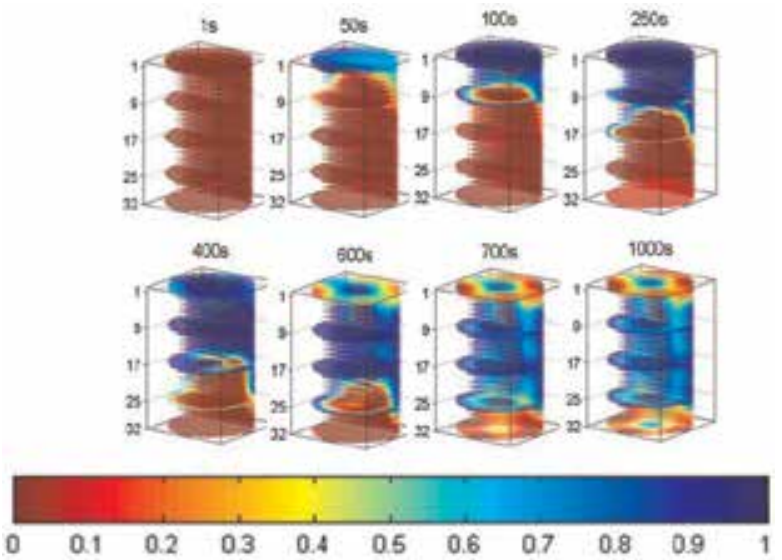

(a)

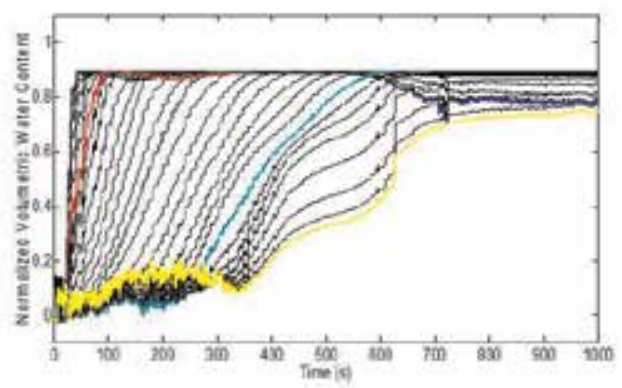

(b)

Figure 6.

Result from experiment 1, soil water infiltration (a) and normalized volumetric water content of soil (blue line, 1st layer; red line, gth layer; green line, 17 th layer; cyan line, 25 th layer; and yellow line, 32 nd layer) (b).

Topp and Malicki models. However, the second model looks similar to Roth et al. [21] model, which gives a linear relationship between normalized volumetric water content and relative permittivity. In contrast, the third model is out of fit with other models. 
Dielectric Analysis Model for Measurement of Soil Moisture Water Content Using Electrical... DOI: http://dx.doi.org/10.5772/intechopen.89057

Modification to the first model (Eq. (23a)) to fit with previous models can be done by adding the constants by trial and error. The modification is shown below:

$$
\Theta=0.9 \times\left(\varepsilon_{N^{-}}-0.015\right)^{0.65}
$$

Figure 5 shows the first model with modification (Eq. (25)) quite fit with the Topp and Malicki models.

Based on this result, Eq. (25) was used to analyze the normalized volumetric water content during water infiltration in soil. The results of water infiltration in soil can be seen in Figure 6. Figure 6a shows the images of normalized volumetric water content from red (i.e., dry condition, $\varepsilon_{N}=0$ ) to blue (i.e., saturated condition, $\left.\varepsilon_{N}=1\right)$ colors. The scale of the color means normalization value of relative permittivity distribution in image.

Figure 6a shows the image sequencing of water infiltration methods from 1,50 , $100,250,400,600,700$, and $1000 \mathrm{~s}$, respectively. In this figure, the position and movement of water per second can be seen clearly. Figure $\mathbf{6 b}$ shows normalized

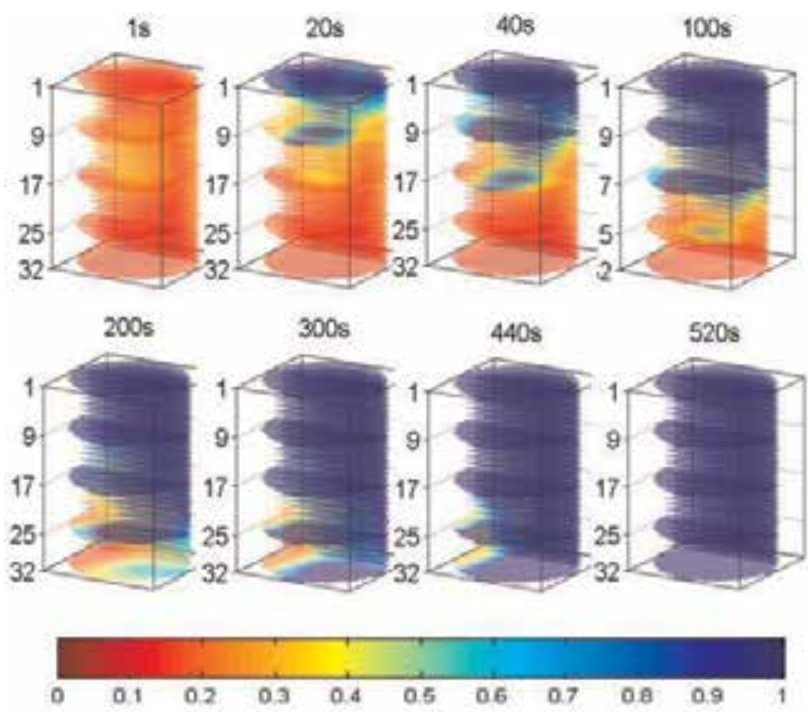

(a)

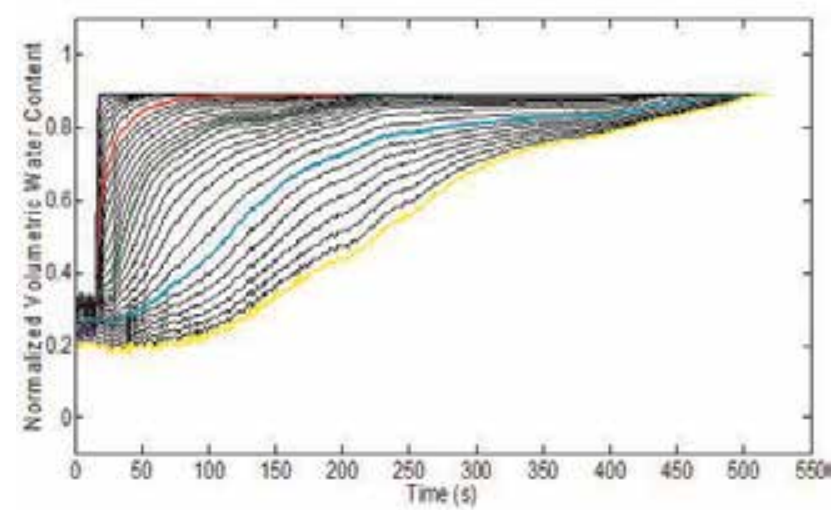

(b)

Figure 7.

Result from experiment 2, soil water infiltration (a) and normalized volumetric water content of soil (blue line, 1st layer; red line, gth layer; green line, 17th layer; cyan line, 25 th layer; and yellow line, 32 nd layer) (b). 
volumetric water content of soil for 32 layers during water infiltration in the soil. Blue, green, red, cyan, and yellow lines indicated 1, 9, 17, 25, and 32 soil layers, respectively. In the first layer, the normalized volumetric water content increases very fast and reaches a saturated condition at around 100-300 s. The degree of saturation for 1st layer starts to decrease after supplying of water was stopped at $300 \mathrm{~s}$ and relative constant was at around 0.3 from 700 to $1000 \mathrm{~s}$. For 9th and 17th layers, the degree of saturation increases sequencing and reaches a saturated condition at a similar time (i.e., at around $450 \mathrm{~s}$ ) and starts to decrease at $500 \mathrm{~s}$, while for the 25th layer, the degree of saturation increases at around $300 \mathrm{~s}$ and reaches a stable condition at $700 \mathrm{~s}$ with a degree of saturation at around 0.85 . Moreover at the $32 \mathrm{nd}$ layer, the degree of saturation increases at $630 \mathrm{~s}$, and the degree of saturation reaches 0.2 at the end of the experiment (i.e., $1000 \mathrm{~s}$ ). This experiment showed clearly the availability of air trapped at the bottom of soil in the vessel (see Figure 6a).

Figure 7 also shows the image sequencing of water infiltration (Figure 7a) and normalized volumetric water content of each layer of the soil column (Figure 7b). In Figure 7a, it can be seen that the silty sand has a normalized relative permittivity value around 0.3 before water infiltrate to the soil column. It can be caused by two possibilities, (i) because of the moisture content stored in the soil and (ii) because the soil particles are very small and compact, so the porosity of soil is also small which causes no air cavity in the soil. From Figure $7 \mathbf{b}$ we can see how the water infiltrates into the each layer clearly. The first layer has increased drastically around the first $30 \mathrm{~s}$. It is easily understood that the top layers will reach the maximum value of normalized volumetric water content first, because these layers get the first supply of water.

\section{Conclusion}

Some equations between the relative permittivity and volumetric water content have been described in this study. From this study, there is an equation that has demonstrated efficacy in conformity with the data of experimental results, that is, equation proposed by [32]. This equation uses the porosity factor as a parameter in the relationship between volumetric water content and relative permittivity.

Normalized volumetric water content of soil has been analyzed in this study using ECVT system. Normalized volumetric water content can be shown and analyzed layer per layer of soil column for every second. We found that the ECVT system has advantages in measuring soil water content which are nondestructive and noninvasive to the sample object, 3D image, and real-time monitoring for water infiltration.

\section{Acknowledgements}

The authors wish to thank the Ministry of Research, Technology and Higher Education of Republic of Indonesia, for providing the research grand.

\section{Conflict of interest}

The authors declare that there is no conflict of interest. 
Dielectric Analysis Model for Measurement of Soil Moisture Water Content Using Electrical... DOI: http://dx.doi.org/10.5772/intechopen.89057

\section{Author details}

Mukhlisin Muhammad ${ }^{1 *}$ and Saputra Almushfi ${ }^{2}$

1 Politeknik Negeri Semarang, Semarang, Indonesia

2 CTECH Labs Edwar Technology, Tangerang, Indonesia

*Address all correspondence to: mmukhlis2@gmail.com

\section{IntechOpen}

(C) 2019 The Author(s). Licensee IntechOpen. This chapter is distributed under the terms of the Creative Commons Attribution License (http://creativecommons.org/licenses/ by/3.0), which permits unrestricted use, distribution, and reproduction in any medium, provided the original work is properly cited. (cc) BY 


\section{References}

[1] Moroizumi T, Hamada H, Sukchan S, Ikemoto M. Soil water content and water balance in rainfed fields in Northeast Thailand. Agricultural Water Management. 2009;96(1):160-166

[2] Kottler BD, White JC, Kelsey JW. Influence of soil moisture on the sequestration of organic compounds in soil. Chemosphere. 2001;42(8):893-898

[3] Osman N, Barakbah SS. Parameters to predict slope stability-Soil water and root profiles. Ecological Engineering. 2006;28(1):90-95

[4] Mukhlisin M, Taha MR, Kosugi K. Numerical analysis of effective soil porosity and soil thickness effects on slope stability at a hillslope of weathered granitic soil formation. Geosciences Journal. 2008;12(4):401-410

[5] Mukhlisin M, Taha MR. Slope stability analysis of a weathered granitic hillslope as effects of soil thickness. European Journal of Scientific Research. 2009;30(1):36-44

[6] Mukhlisin M, Baidillah MR, Taha MR, El-Shafie A. Effect of soil water retention model on slope stability analysis. International Journal of Physical Sciences. 2011;6(19):4629-4635

[7] Topp GC. State of the art of measuring soil water content. Hydrological Processes. 2003;17(14): 2993-2996

[8] Huisman JA, Hubbard SS, Redman JD, Annan AP. Measuring soil water content with ground penetrating radar: A review. Vadose Zone Journal. 2003; 2(4):476-491

[9] Basinger JM, Kluitenberg GJ, Ham JM, Frank JM, Barnes PL, Kirkham MB. Laboratory evaluation of the dual-probe heat-pulse method for measuring soil water content. Vadose Zone Journal. 2003;2(3):389
[10] Gaskin GJ, Miller JD. Measurement of soil water content using a simplified impedance measuring technique. Journal of Agricultural Engineering Research. 1996;63(2):153-159

[11] Ramírez V, Sánchez JA, Hernández G, Solís S, Torres J, Antaño R, et al. A promising electrochemical test for evaluating the hydrocarbon-type pollutants contained in industrial waste soils. International Journal of Electrochemical Science. 2011;6(5): 1415-1437

[12] Ruíz C, Anaya J, Ramírez V, Alba GI, García MG, Carrillo-Chávez A, et al. Soil Arsenic Removal by a Permeable Reactive Barrier of Iron Coupled to an Electrochemical Process. 2011

[13] Wu SY, Zhou QY, Wang G, Yang L, Ling CP. The relationship between electrical capacitance-based dielectric constant and soil water content.

Environment and Earth Science. 2011; 62(5):999-1011

[14] Robinson DA, Jones SB, Wraith JM, Or D, Friedman SP. A review of advances in dielectric and electrical conductivity measurement in soils using time domain reflectometry. Vadose Zone Journal. 2003;2(4):444-475

[15] Gardner CMK, Dean TJ, Cooper JD. Soil water content measurement with a high-frequency capacitance sensor. Journal of Agricultural Engineering Research. 1998;71(4):395-403

[16] Warsito W, Marashdeh Q, Fan L-S. Electrical capacitance volume tomography. IEEE Sensors Journal. 2007;7(4):525-535

[17] Warsito W, Marashdeh Q, Fan LS. Real time volumetric imaging of multiphase flows using electrical capacitance volume-tomography 
(ECVT). In: 5th World Congress in Industrial Process Tomography; 2007. pp. $755-760$

[18] Mukhlisin M, Baidillah MR, El-Shafie A, Taha MR. Real time monitoring of soil water infiltration using electrical capacitance volumetomography (ECVT). In: 4th International Conference on Water Resources and Arid Environments (ICWRAE 4); 2010. pp. 749-756

[19] Yang WQ, Spink DM, York TA, McCann H. An image-reconstruction algorithm based on Landweber's iteration method for electricalcapacitance tomography. Measurement Science and Technology. 1999;10(11): 1065-1069

[20] Topp GC, Davis JL, Annan AP. Electromagnetic determination of soil water content: Measurements in coaxial transmission lines. Water Resources Research. 1980;16(3):574-582

[21] Roth CH, Malicki MA, Plagge R. Empirical evaluation of the relationship between soil dielectric constant and volumetric water content as the basis for calibrating soil moisture measurements by TDR. Journal of Soil Science. 1992; 43(1):1-13

[22] Malicki MA, Plagge R, Renger M, Walczak RT. Application of timedomain reflectometry (TDR) soil moisture miniprobe for the determination of unsaturated soil water characteristics from undisturbed soil cores. Irrigation Science. 1992;13(2): 65-72

[23] Ferré PA, Rudolph DL, Kachanoski RG. Spatial averaging of water content by time domain reflectometry: Implications for twin rod probes with and without dielectric coatings. Water Resources Research. 1996;32(2):271-279

[24] Schaap MG, de Lange L, Heimovaara TJ. TDR calibration of organic forest floor media. Soil Technology. 1997;11(2):205-217

[25] Curtis JO. Moisture effects on the dielectric properties of soils. IEEE Transactions on Geoscience and Remote Sensing. 2001;39(1):125-128

[26] Lundien JR. Terrain Analysis by Electromagnetic Means. Vicksburg, MS: U.S. Army Engineer Waterways Experiment Station; 1971

[27] Newton RW. Microwave Remote Sensing and its Application to Soil Moisture Detection. College Station, TX: Texas A\&M University, College Station; 1977

[28] Wang J, Schmugge T, Williams D. Dielectric constants of soils at microwave frequencies-II. National Aeronautics and Space Administration Technical Paper. 1978

[29] Wang JR, Schmugge TJ. An empirical model for the complex dielectric permittivity of soils as a function of water content. IEEE Transactions on Geoscience and Remote Sensing. 1980;GE-18(4):288-295

[30] Roth K, Schulin R, Fluhler H, Attinger W. Calibration of time domain reflectometry for water content measurement using a composite dielectric approach. Water Resources Research. 1990;26:2267-2273

[31] Dobson MC, Ulaby FT, Hallikainen MT, El-Rayes MA. Microwave dielectric behavior of wet soil-Part II: Dielectric mixing models. IEEE Transactions on Geoscience and Remote Sensing. 1985; GE-23:35-45

[32] Malicki MA, Plagge R, Roth CH. Improving the calibration of dielectric TDR soil moisture determination taking into account the solid soil. European Journal of Soil Science. 1996;47:357-366

[33] Friedman SP. A saturation degreedependent composite spheres model for 
describing the effective dielectric constant of unsaturated porous media. Water Resources Research. 1998;34: 2949-2961

[34] Hilhorst MA, Dirksen C, Kampers FWH, Feddes RA. New dielectric mixture equation for porous materials based on depolarization factors. Soil Science Society of America Journal. 2000;64:1581-1587

[35] Robinson DA, Jones SB, Blonquist JM, Friedman SP. A physically derived water content/permittivity calibration model for coarse-textured, layered soils. Soil Science Society of America Journal. 2005;69:1372-1378 


\title{
Techniques for Calculating Two Interesting Types of Dielectric Materials in Straight Rectangular Waveguides and Their Applications
}

\author{
Zion Menachem
}

\begin{abstract}
This chapter presents two interesting types of dielectric materials in the straight rectangular waveguides. Five examples of the different discontinuous cross sections and complementary shapes will demonstrate. We will introduce in all case the effective technique to calculate the dielectric profile in the cross section. The first type will demonstrate where the dielectric material is located in the center of the cross section. The second type will demonstrate where the hollow core is located in the center of the cross section in the case of the hollow waveguide. The two different types are complementary shapes for two different applications. The proposed techniques relate to the method based on Laplace and Fourier transforms and the inverse Laplace and Fourier transforms. The method is based also on Fourier transform, thus we need use with the image method to calculate the dielectric profile in the cross section. The image method and periodic replication are needed for fulfilling the boundary condition of the metallic waveguide. The applications are useful for straight waveguides in millimeter regimes, in the cases where the dielectric profile is located in the center of the cross section, for cases where the hollow rectangle is located in the center of the cross section, and also for complicated and discontinuous profiles in the cross section.
\end{abstract}

Keywords: wave propagation, dielectric profiles, rectangular waveguides, dielectric materials

\section{Introduction}

Review of numerical and approximate methods for the modal analysis of general optical dielectric waveguides with emphasis on recent developments has been published [1]. In this review, interesting methods are given such as, the finitedifference and the finite-element methods. Review of numerical methods for the analysis of the homogeneous and inhomogeneous, isotropic and anisotropic, microwave and optical dielectric waveguides with arbitrarily-shaped cross sections has been published [2]. The main approaches as the integral equations, finite difference, and finite element have been discussed. 
A fundamental and accurate approach to compute the attenuation of electromagnetic waves propagating has been proposed [3]. The propagation constant was found by substituting the values of transverse wave numbers into the dispersion relation. A Green's dyadic for describing the propagating electromagnetic waves in a rectangular dielectric waveguide has been developed [4]. The use of Green's dyadic developed in order to calculate the effect of small perturbations upon the system.

An analytical method for solution of one-dimensional optical systems, based on the differential transfer matrices has been presented [5]. An approach to solve the problem of the propagation of electromagnetic waves in unidimensional media with an arbitrary variation of their dielectric permittivity has been proposed [6]. This method was deduced from the Maxwell equations with a minimum of approximations and allows a full vectorial description of both the electric and magnetic fields through the direct calculation of their cartesian coordinates. The equations permit the simulation of materials with a continuous variation of their dielectric permittivity without approximating them by discontinuous layered media, reducing so the computational effort of the models.

An analytical method for solution of non-homogeneous anisotropic optical systems, based on the extension of transfer matrices into differential form has been presented [7]. This approach can be used for exact calculation of various functions including reflection and transmission coefficients, band structures and bound states. A full-wave analysis of lossy dielectric waveguides using a hybrid vector finite element method has been presented [8]. The direct matrix solution technique with minimum degree of reordering has been combined with the modified Lanczos algorithm to solve for the resultant sparse generalized eigenmatrix equation efficiently.

Three-dimensional finite-element method with edge elements for electromagnetic waveguide discontinuities has been proposed [9]. This paper shows that the finite-element method using edge elements succeeds in suppressing spurious solutions and moreover that this method succeeds in the analysis of three-dimensional electromagnetic waveguide problems with metal wedges. An analytical approach based on scalar wave approximation to estimate the modal dispersion characteristics and cutoff condition of an optical waveguide has been presented [10]. This approach has an arbitrary and uniform core cross-section. The structure represents the core of a circular waveguide which is compressed at both the ends of a diameter.

Propagation characteristics of modes in some rectangular waveguides using the finite-difference time-domain method have been studied [11]. The method in this paper was used to determine the modal characterization of rectangular waveguide structures by means of a least-square non-linear fitting to a theoretical modal expansion. Wave propagation along a rectangular waveguide with slowly varying width has been investigated [12] with the help of field theory and approximate circuit theory. Many properties of the modulated periodic structure, e.g., the frequency dependence of the propagation constant, group and phase velocities, and the electric field axial variation for the fundamental space harmonic and its filterlike property have been investigated.

A method for measuring samples using a partially-filled waveguide has been presented [13]. A mode-matching technique was used to determine the fields in the three regions. An advantageous finite element method for the rectangular waveguide problem has been developed [14] by which complex propagation characteristics may be obtained for arbitrarily shaped waveguide.

A method has been introduced for the frequency domain analysis of arbitrary longitudinally inhomogeneous waveguides [15]. The integral equations of the longitudinally inhomogeneous waveguides, converted from their differential equations, were solved using the method of moments. A method has been introduced for the frequency domain analysis of arbitrarily loaded lossy and dispersive 
nonuniform transmission lines [16]. In this method, all distributed primary parameters of the line and also the voltage and current distribution along the line were considered as a Taylor's series.

A technique based on the two-dimensional Fourier transform has been presented [17] and applied to the study of nonlinear wave propagation phenomena in one-dimensional, finite, nonlinear transmission lines.

A method for calculating the modes of arbitrarily shaped dielectric waveguides has been presented [18]. It consists of expanding the field in a two-dimensional Fourier series. The expansion has been used to convert the scalar wave equation into a matrix eigenvalue equation. A transfer matrix function for the analysis of electromagnetic wave propagation along the straight dielectric waveguide with arbitrary profiles has been proposed [19]. This method is based on the Laplace and Fourier transforms and the inverse Laplace and Fourier transforms.

\section{Complicated and discontinuous profiles in the cross section}

In this chapter, we present some examples of dielectric structures as shown in Figure 1a-e. The method is based on Fourier transform, thus we need use with the image method and periodic replication for fulfilling the boundary conditions of the metallic waveguide. We relate also to the complementary shapes for different applications. The periodicity and the symmetry properties are chosen to force the boundary conditions at the location of the walls in real problem, by extending the

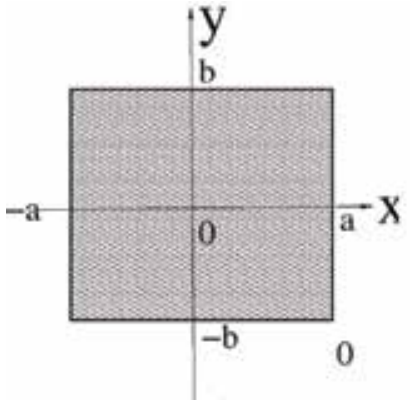

(a)

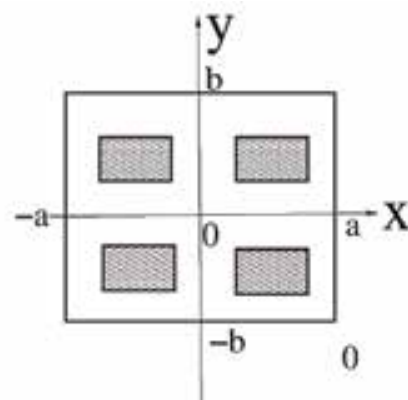

(b)

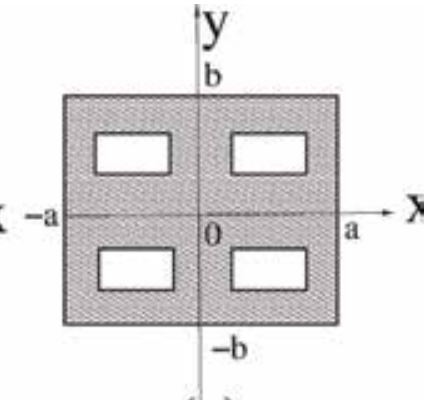

(c)

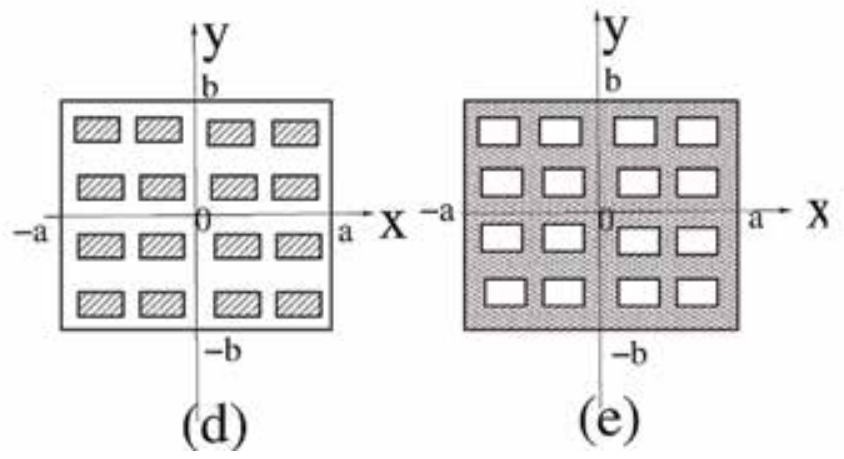

Figure 1.

The image method and periodic replication for five examples. (a). The cross section entirely filled with the dielectric material, $(b)$. The dielectric material is located in the center of the cross section, $(c)$. The hollow waveguide where the hollow rectangle is located in the center of the cross section, $(d)$. The cross section consists with four dielectric profiles, and (e). The hollow waveguide with four hollow rectangles. 


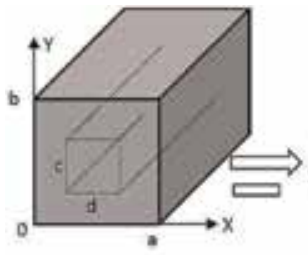

(a)

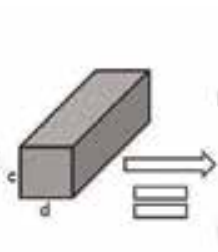

(b)

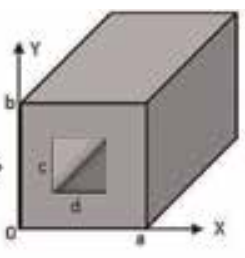

(c)

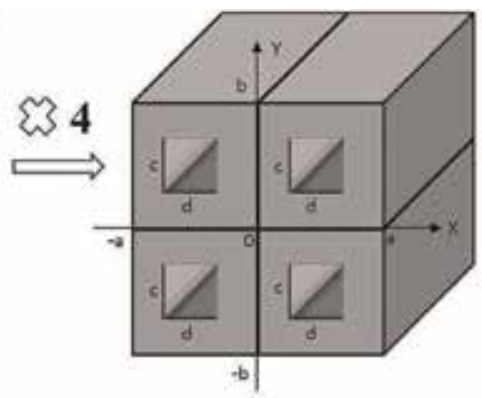

(d)

Figure 2.

Example of receiving a hollow waveguide where the hallow rectangle is located in the center (c) by substracting the waveguide with dielectric material located at the center of the cross section (b) from the waveguide entirely filled with the dielectric material (a), and by using the image method $(d)$.

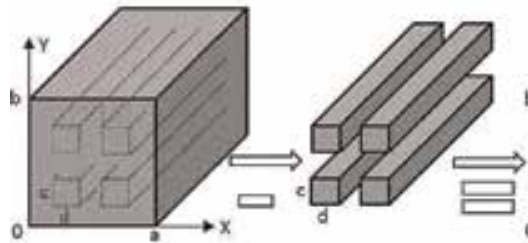

(a)

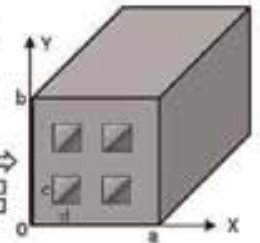

(c)

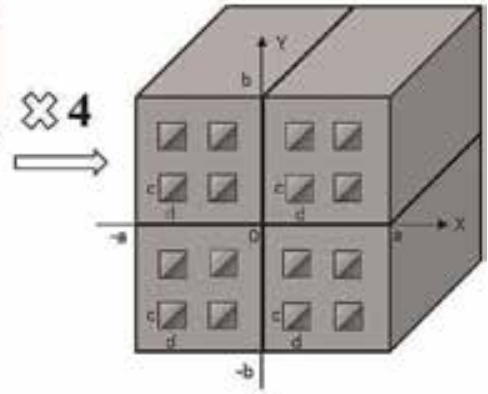

(d)

Figure 3.

Example of receiving a hollow waveguide with four hollow rectangles (c) by substracting the four dielectric rectangles (b) from the waveguide entirely filled with the dielectric material (a), and by using the image method $(d)$.

waveguide region $(0 \leq x \leq a$, and $0 \leq y \leq b)$ to a fourfold larger regions ( $-a \leq x \leq a$, and $-b \leq y \leq b)$, as larger regions, as described in [19]. Figure 1a shows the case where the cross section entirely filled with the dielectric material. The dielectric material in Figure 1b is located in the center of the cross section. Figure 1c represents the hollow waveguide where the hollow rectangle is located in the center of the cross section. The cross section of Figure 1d consists with four dielectric profiles in the cross section. Figure 1e represents the hollow waveguide with four hollow rectangles.

Note that the geometries from Figure 1a-e become more complex. Calculating of the dielectric profile for Figure 1a is the simplest and for Figure 1e is the most complicated in relation to the examples described in these examples. The integrals of the dielectric profiles of the geometries described in Figure 1a and $\mathbf{b}$ can be calculated by analytical form and without solving numerical form. The calculation of the dielectric profile for the geometry of Figure 1c for a hollow rectangle in the center is interesting in the case of hollow waveguide, but it is more complicated than the case of Figure $1 \mathbf{b}$. Therefore, in this case we should calculate the dielectric profile by substracting the dielectric profile of Figure 1b from the dielectric profile of Figure 1a. The calculation of the integrals of the dielectric profiles for the geometries depicted in Figure 1d and e are already more complicated, and in such cases the proposed techniques require that the integrals be solved numerically. We will explain the proposed technique for calculating the dielectric profile for each case.

Figure 2c represents an example of receiving a hollow waveguide where the hollow rectangle is located in the center by substracting the waveguide with dielectric material 
located at the center of the cross section (Figure $2 \mathbf{b}$ ) from the waveguide entirely filled with the dielectric material (Figure 2a), and by using the image method (Figure 2d). Figure $3 \mathrm{c}$ represents an example of receiving a hollow waveguide with four hollow rectangles by substracting the four dielectric rectangles (Figure $3 \mathbf{b}$ ) from the waveguide entirely filled with the dielectric material (Figure 3a), and by using with the image method (Figure 3d).

\section{The analytical technique to calculate the dielectric profiles}

This section presents a technique to calculate the dielectric profile for the two inhomogeneous geometries of the cross section, as shown in Figure $\mathbf{4 a}$ and $\mathbf{b}$.

Figure $\mathbf{4 a}$ and $\mathbf{b}$ show the cross section of Figure $\mathbf{1 b}$ and $\mathbf{c}$, respectively, with the relevant parameters. These figures represent two examples of the complementary shapes in the cross section. The periodic replication is shown in Figure $\mathbf{4 c}$ by using with the image method. The dielectric material in Figure $\mathbf{4 a}$ is located in the center of the cross section. The hollow rectangle in Figure $\mathbf{4 b}$ is located in the center of the cross section in the case of the hollow waveguide.

The dielectric profile $g(x, y)$ is given according to $\epsilon(x, y)=\epsilon_{0}(1+g(x, y))$. If $g(x, y)=g_{0}$ and $\epsilon_{r}(x, y)=\epsilon_{r}$, then we obtain that $g 0=\epsilon_{r}-1$. According to

Figure 4a and $\mathbf{c}$ for $g(x, y)=g_{0}$, we obtain

$$
\begin{aligned}
& g(n, m)=\frac{g_{0}}{4 a b} \int_{-a}^{a} d x \int_{-b}^{b} \exp \left[-j\left(k_{x} x+k_{y} y\right)\right] d y \\
&=\frac{g_{0}}{4 a b}\left\{\int_{x_{11}}^{x_{12}} d x \int_{y_{11}}^{y_{12}} \exp \left[-j\left(k_{x} x+k_{y} y\right)\right] d y+\int_{-x_{12}}^{-x_{11}} d x \int_{y_{11}}^{y_{12}} \exp \left[-j\left(k_{x} x+k_{y} y\right)\right] d y\right. \\
&\left.+\int_{-x_{12}}^{-x_{11}} d x \int_{-y_{12}}^{-y_{11}} \exp \left[-j\left(k_{x} x+k_{y} y\right)\right] d y+\int_{x_{11}}^{x_{12}} d x \int_{-y_{12}}^{-y_{11}} \exp \left[-j\left(k_{x} x+k_{y} y\right)\right] d y\right\} \\
&=\frac{g_{0}}{4 a b}\left\{\int_{x_{11}}^{x_{12}} d x \int_{y_{11}}^{y_{12}} \exp \left[-j\left(k_{x} x+k_{y} y\right)\right] d y+\int_{x_{12}}^{x_{11}}-d x \int_{y_{11}}^{y_{12}} \exp \left[-j\left(-k_{x} x+k_{y} y\right)\right] d y\right. \\
&\left.+\int_{x_{12}}^{x_{11}}-d x \int_{y_{12}}^{y_{11}}-\exp \left[-j\left(-k_{x} x-k_{y} y\right)\right] d y+\int_{x_{11}}^{x_{12}} d x \int_{y_{12}}^{y_{11}}-\exp \left[-j\left(k_{x} x-k_{y} y\right)\right] d y\right\} \\
&=\frac{g_{0}}{4 a b}\left\{\int_{x_{11}}^{x_{12}} \exp \left[j\left(k_{x} x\right)\right] d x \int_{y_{11}}^{y_{12}} \exp \left[j\left(k_{y} y\right)\right] d y+\int_{x_{11}}^{x_{12}} \exp \left[j\left(k_{x} x\right)\right] d x \int_{y_{11}}^{y_{12}} \exp \left[-j\left(k_{y} y\right)\right] d y\right. \\
&\left.+\int_{x_{11}}^{x_{12}} \exp \left[-j\left(k_{x} x\right)\right] d x \int_{y_{11}}^{y_{12}} \exp \left[j\left(k_{y} y\right)\right] d y+\int_{x_{11}}^{x_{12}} \exp \left[-j\left(k_{x} x\right)\right] d x \int_{y_{11}}^{y_{12}} \exp \left[-j\left(k_{y} y\right)\right] d y\right\} \\
&=\frac{g_{0}}{4 a b}\left\{\int_{x_{11}}^{x_{12}} d x \int_{y_{11}}^{y_{12}} \exp \left[-j\left(k_{x} x+k_{y} y\right)\right] d y+\int_{x_{11}}^{x_{12}} d x \int_{y_{11}}^{y_{12}} \exp \left[j\left(k_{x} x-k_{y} y\right)\right] d y\right. \\
&\left.+\int_{x_{11}}^{x_{12}} d x \int_{y_{11}}^{y_{12}} \exp \left[j\left(k_{x} x+k_{y} y\right)\right] d y+\int_{x_{11}}^{x_{12}} d x \int_{y_{11}}^{y_{12}} \exp \left[-j\left(k_{x} x-k_{y} y\right)\right] d y\right\} \\
& \frac{g_{0}}{2 a b}\left\{\int_{x_{11}}^{x_{12}}\left(\exp \left(j k_{x} x\right)+\exp \left(-j k_{x} x\right)\right) d x \int_{y_{12}}^{y_{11}} \cos \left(k_{y} y\right) d y\right\} \\
& \\
&
\end{aligned}
$$




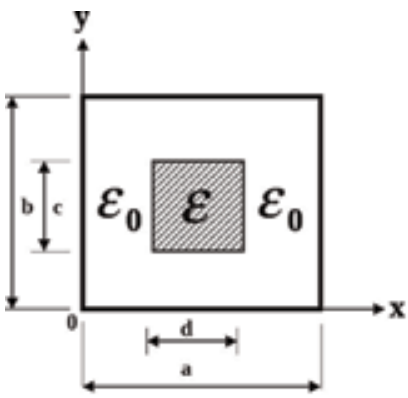

(a)

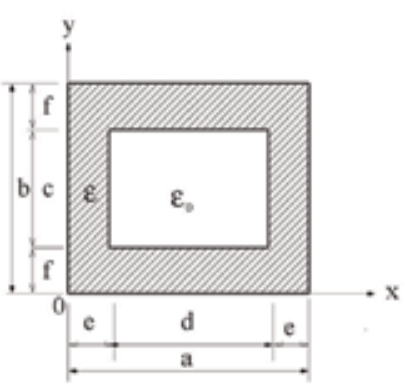

(b)

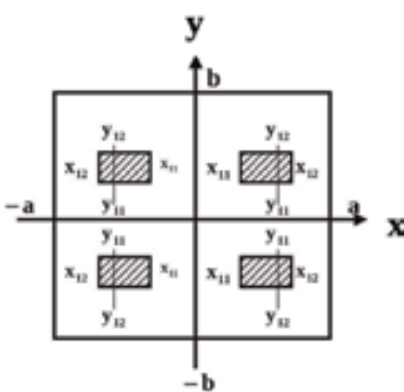

(c)

Figure 4 .

Two examples of the complementary shapes of profiles in the cross section and the periodic replication, (a). The dielectric material is located in the center of the cross section with the relevant parameters of Figure $\mathbf{1} b,(b)$. The hollow rectangle is located in the center of the cross section in the case of the hollow waveguide with the relevant parameters of Figure 1c, (c). The periodic replication according to the image method.

where $k_{x}=(n \pi x) / a$, and $k_{y}=(m \pi y) / b$. The derivatives of the dielectric profile are calculated according to $g_{x}(x, y)=(1 / \epsilon(x, y))(d \epsilon(x, y) / d x)$, and $g_{y}(x, y)=(1 / \epsilon(x, y))(d \epsilon(x, y) / d y)$, where $\epsilon(x, y)=\epsilon_{0}(1+g(x, y))$.

For the cross section as shown in Figure 4a and according to Figure 4c, the center of the dielectric rectangle is located at $(\mathrm{a} / 2, \mathrm{~b} / 2)$. According to Figure $4 \mathbf{a}$ and c, the Fourier components of the dielectric profile are given by

$$
g_{1}(n, m)= \begin{cases}\frac{g_{0}}{4 a b}(4 c d) & n=0, m=0 \\ \frac{g_{0}}{4 a b}\left(\frac{8 d}{k_{0 y} m} \sin \left(\frac{k_{0 y} m c}{2}\right) \cos \left(\frac{k_{0 y} m b}{2}\right)\right) & n=0, m \neq 0 \\ \frac{g_{0}}{4 a b}\left(\frac{8 c}{k_{0 x} n} \sin \left(\frac{k_{0 x} n d}{2}\right) \cos \left(\frac{k_{0 x} n a}{2}\right)\right) & n \neq 0, m=0 \\ \frac{g_{0}}{4 a b}\left(\frac{16}{k_{0 x} k_{0 y} n m} \sin \left(\frac{k_{0 x} n d}{2}\right) \cos \left(\frac{k_{0 x} n a}{2}\right) \sin \left(\frac{k_{0 y} m c}{2}\right) \cos \left(\frac{k_{0 y} m b}{2}\right)\right) & n \neq 0, m \neq 0\end{cases}
$$

Similarly, for the cross section entirely filled with the dielectric material as shown in Figure 1a, the Fourier components of the dielectric profile are given by

$$
g_{2}(n, m)=\left\{\begin{array}{ll}
g_{0} & n=0, m=0 \\
\frac{g_{0}}{4 a b}\left(\frac{8 a}{k_{0 y} m} \sin \left(\frac{k_{0 y} m b}{2}\right) \cos \left(\frac{k_{0 y} m b}{2}\right)\right) & n=0, m \neq 0 \\
\frac{g_{0}}{4 a b}\left(\frac{8 b}{k_{0 x} n} \sin \left(\frac{k_{0 x} n a}{2}\right) \cos \left(\frac{k_{0 x} n a}{2}\right)\right) & n \neq 0, m=0 \\
\frac{g_{0}}{4 a b}\left(\frac{16}{k_{0 x} k_{0 y} n m} \sin \left(\frac{k_{0 x} n a}{2}\right) \cos \left(\frac{k_{0 x} n a}{2}\right) \sin \left(\frac{k_{0 y} m b}{2}\right) \cos \left(\frac{k_{0 y} m b}{2}\right)\right) & n \neq 0, m \neq 0
\end{array} .\right.
$$

The Fourier components of the dielectric profile $(g(n, m))$ of the hollow rectangular waveguide with the hollow rectangle in the center of the cross section 
(Figure 4b) are calculated by subtracting the dielectric profile of the waveguide with the dielectric material in the core $\left(g_{1}(n, m)\right)$ as shown in Figure 4a from the dielectric profile of the waveguide entirely with the dielectric material $\left(g_{2}(n, m)\right)$ as shown in Figure 1a. Namely, the Fourier components of the dielectric profile $(g(n, m))$ are given by $g_{2}(n, m)-g_{1}(n, m)$. The two different types of the cross sections are complementary shapes for two different applications.

\section{The numerical technique to calculate the dielectric profiles}

\subsection{Calculation of the dielectric profile according to the $\omega_{\varepsilon}$ function}

Figure $5 \mathbf{a}$ and $\mathbf{b}$ show the cross section of Figure 1d and e, respectively, with the relevant parameters. These figures represent two examples of the complementary shapes in the cross section. The cross section consists with four dielectric profiles as shown in Figure 5a. The hollow waveguide with four hollow rectangles is shown in Figure 5b. The centers of the first, the second, the third and the firth dielectric rectangles in Figure 5a and hollow rectangles in Figure $5 \mathbf{b}$ are located at the points $(\mathrm{a} / 4, \mathrm{~b} / 4),(3 \mathrm{a} / 4, \mathrm{~b} / 4),(\mathrm{a} / 4,3 \mathrm{~b} / 4)$, and $(3 \mathrm{a} / 4,3 \mathrm{~b} / 4)$, respectively. The calculation of the elements of the matrix in Figure $5 \mathbf{b}$ is already more complicated. Thus, the Fourier components of the dielectric profile $(g(n, m))$ of the hollow waveguide with four hollow rectangles in Figure $5 \mathbf{b}$ are calculated by subtracting the dielectric profile of the cross section with four dielectric profiles in Figure 5a from the dielectric profile of the waveguide entirely with the dielectric material $\left(g_{2}(n, m)\right)$ as shown in Figure 1a, and according to Eq. (3).

In order to calculate the elements of the dielectric profiles of the inhomogeneous geometry of the cross section in Figure 5a, we can use with the $\omega_{\varepsilon}$ function [20], as shown in Figure 6. The $\omega_{\varepsilon}$ function is defined as $\omega_{\varepsilon}(r)=C_{\varepsilon} \exp \left[-\varepsilon^{2} /\left(\varepsilon^{2}-|r|^{2}\right)\right]$ for $|r|>\varepsilon$, where $C_{\varepsilon}$ is a constant, and $\int \omega_{\varepsilon}(r) d r=1$. In the limit $\varepsilon \rightarrow 0$, the $\omega_{\varepsilon}$ function is shown in Figure 6.

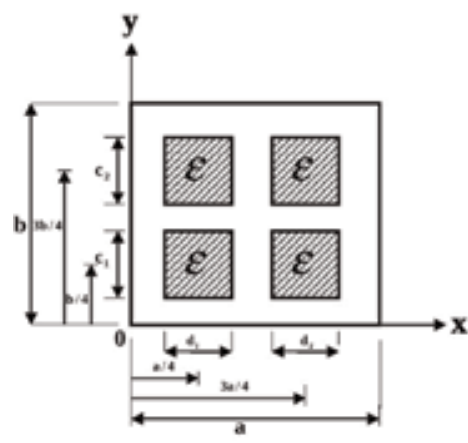

(a)

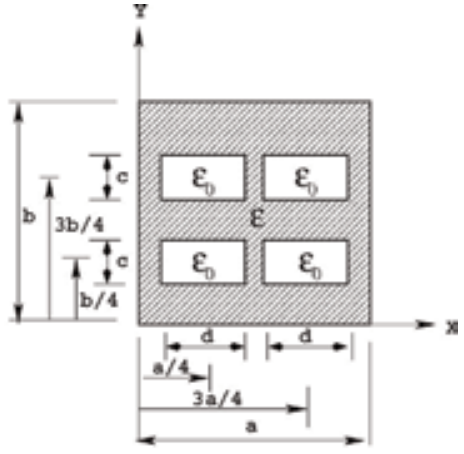

(b)

Figure 5.

Two examples of the complementary shapes of profiles in the cross section and their relevant parameters. The centers of the first, the second, the third and the firth rectangles are located at the points $(a / 4, b / 4),(3 a / 4, b / 4)$, $(a / 4,3 b / 4)$, and $(3 a / 4,3 b / 4)$, respectively: (a) the cross section consists with four dielectric profiles with the relevant parameters of Figure 1d; $(b)$ the hollow waveguide with four hollow rectangles with the relevant parameters of Figure 1e. 


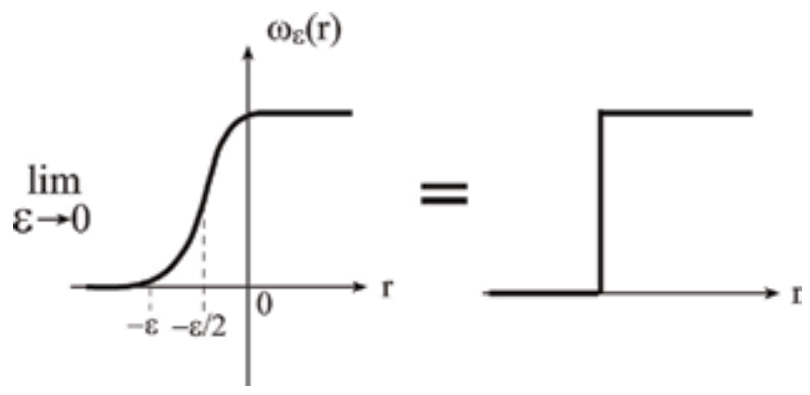

Figure 6.

The $\omega_{\varepsilon}$ function to calculate the elements of the dielectric profiles of the inhomogeneous geometry.

\subsection{The technique based on $\omega_{\varepsilon}$ function for the inhomogeneous cross section}

It is good idea to calculate the dielectric profile of Figure 4a again, by using the $\omega_{\varepsilon}$ function, and before considering to the more complicated geometry as shown in Figure 5a. The second way to calculate the dielectric profile of Figure 4a is given according to the $\omega_{\varepsilon}$ function, where the dielectric profile is located at $(\mathrm{a} / 2, \mathrm{~b} / 2)$ and is given by

$$
g(x)= \begin{cases}g_{0} \exp \left(1-g_{1}(x)\right) & (a-d-\varepsilon) / 2 \leq x<(a-d+\varepsilon) / 2 \\ g_{0} & (a-d+\varepsilon) / 2<x<(a+d-\varepsilon) / 2 \\ g_{0} \exp \left(1-g_{2}(x)\right) & (a+d-\varepsilon) / 2 \leq x<(a+d+\varepsilon) / 2 \\ 0 & \text { else }\end{cases}
$$

and

$$
g(y)=\left\{\begin{array}{ll}
g_{0} \exp \left(1-g_{3}(y)\right) & (b-c-\varepsilon) / 2 \leq y<(b-c+\varepsilon) / 2 \\
g_{0} & (b-c+\varepsilon) / 2<y<(b+c-\varepsilon) / 2 \\
g_{0} \exp \left(1-g_{4}(y)\right) & (b+c-\varepsilon) / 2 \leq y<(b+c+\varepsilon) / 2 \\
0 & \text { else }
\end{array},\right.
$$

where

$$
\begin{array}{ll}
g_{1}(x)=\frac{\varepsilon^{2}}{\varepsilon^{2}-[x-(a-d+\varepsilon) / 2]^{2}}, & g_{2}(x)=\frac{\varepsilon^{2}}{\varepsilon^{2}-[x-(a+d-\varepsilon) / 2]^{2}}, \\
g_{3}(y)=\frac{\varepsilon^{2}}{\varepsilon^{2}-[y-(b-c+\varepsilon) / 2]^{2}}, & g_{4}(y)=\frac{\varepsilon^{2}}{\varepsilon^{2}-[y-(b+c-\varepsilon) / 2]^{2}} .
\end{array}
$$

Similarly, we can calculate the rectangular dielectric profile according to the location of the profile in the cross section of the waveguide.

The elements of the matrix in Fourier space of the inhomogeneous geometry described in Figure $\mathbf{4 a}$ are given in the case of $b \neq c$ by 
Techniques for Calculating Two Interesting Types of Dielectric Materials in Straight Rectangular... DOI: http://dx.doi.org/10.5772/intechopen.88991

$$
\begin{aligned}
g(n, m)= & \frac{g_{0}}{a b}\left\{\int_{(a-d-\varepsilon) / 2}^{(a-d+\varepsilon) / 2} \exp \left(1-g_{1}(x)\right) \cos \left(\frac{n \pi x}{a}\right) d x\right. \\
& \left.+\int_{(a-d+\varepsilon) / 2}^{(a+d-\varepsilon) / 2} \cos \left(\frac{n \pi x}{a}\right) d x+\int_{(a+d-\varepsilon) / 2}^{(a+d+\varepsilon) / 2} \exp \left(1-g_{2}(x)\right) \cos \left(\frac{n \pi x}{a}\right) d x\right\} \\
& \left\{\int_{(b-c-\varepsilon) / 2}^{(b-c+\varepsilon) / 2} \exp \left(1-g_{3}(y)\right) \cos \left(\frac{m \pi y}{b}\right) d y\right. \\
& \left.+\int_{(b-c+\varepsilon) / 2}^{(b+c-\varepsilon) / 2} \cos \left(\frac{m \pi y}{b}\right) d y+\int_{(b+c-\varepsilon) / 2}^{(b+c+\varepsilon) / 2} \exp \left(1-g_{4}(y)\right) \cos \left(\frac{m \pi y}{b}\right) d y\right\} .
\end{aligned}
$$

\subsection{The technique based on $\omega_{\varepsilon}$ function for the inhomogeneous cross section}

Figure 5a shows the cross section where the centers of the first, the second, the third and the firth rectangles are located at the points $(a / 4, b / 4),(3 a / 4, b / 4),(a / 4$, $3 \mathrm{~b} / 4)$, and $(3 \mathrm{a} / 4,3 \mathrm{~b} / 4)$, respectively, and for $\epsilon_{r}=1.1,1.2,1.3$ and 1.4 , respectively. We assume for simplicity that $d_{1}=d_{2}=d$ and $c_{1}=c_{2}=c$, according to Figure 5a.

The dielectric profile of Figure $5 \mathbf{a}$ is given according to the $\omega_{\varepsilon}$ function by

$$
g(x)= \begin{cases}g_{0} \exp \left(1-q_{1}(x)\right) & \left((a / 2)-d_{1}-\varepsilon\right) / 2 \leq x<\left((a / 2)-d_{1}+\varepsilon\right) / 2 \\ g_{0} & \left((a / 2)-d_{1}+\varepsilon\right) / 2<x<\left((a / 2)+d_{1}-\varepsilon\right) / 2 \\ g_{0} \exp \left(1-q_{2}(x)\right) & \left((a / 2)+d_{1}-\varepsilon\right) / 2 \leq x<\left((a / 2)+d_{1}+\varepsilon\right) / 2 \\ g_{0} \exp \left(1-q_{3}(x)\right) & \left((3 a / 2)-d_{2}-\varepsilon\right) / 2 \leq x<\left((3 a / 2)-d_{2}+\varepsilon\right) / 2 \\ g_{0} & \left((3 a / 2)-d_{2}+\varepsilon\right) / 2<x<\left((3 a / 2)+d_{2}-\varepsilon\right) / 2 \\ g_{0} \exp \left(1-q_{4}(x)\right) & \left((3 a / 2)+d_{2}-\varepsilon\right) / 2 \leq x<\left((3 a / 2)+d_{2}+\varepsilon\right) / 2 \\ 0 & \text { else }\end{cases}
$$

and

$$
g(y)= \begin{cases}g_{0} \exp \left(1-q_{5}(y)\right) & \left((b / 2)-c_{1}-\varepsilon\right) / 2 \leq y<\left((b / 2)-c_{1}+\varepsilon\right) / 2 \\ g_{0} & \left((b / 2)-c_{1}+\varepsilon\right) / 2<y<\left((b / 2)+c_{1}-\varepsilon\right) / 2 \\ g_{0} \exp \left(1-q_{6}(y)\right) & \left((b / 2)+c_{1}-\varepsilon\right) / 2 \leq y<\left((b / 2)+c_{1}+\varepsilon\right) / 2 \\ g_{0} \exp \left(1-q_{7}(y)\right) & \left((3 b / 2)-c_{2}-\varepsilon\right) / 2 \leq y<\left((3 b / 2)-c_{2}+\varepsilon\right) / 2 \\ g_{0} & \left((3 b / 2)-c_{2}+\varepsilon\right) / 2<y<\left((3 b / 2)+c_{2}-\varepsilon\right) / 2 \\ g_{0} \exp \left(1-q_{8}(y)\right) & \left((3 b / 2)+c_{2}-\varepsilon\right) / 2 \leq y<\left((3 b / 2)+c_{2}+\varepsilon\right) / 2 \\ 0 & \text { else }\end{cases}
$$

where

$$
\begin{array}{ll}
q_{1}(x)=\frac{\varepsilon^{2}}{\varepsilon^{2}-\left[x-\left((a / 2)-d_{1}+\varepsilon\right) / 2\right]^{2}}, & q_{2}(x)=\frac{\varepsilon^{2}}{\varepsilon^{2}-\left[x-\left((a / 2)+d_{1}-\varepsilon\right) / 2\right]^{2}}, \\
q_{3}(x)=\frac{\varepsilon^{2}}{\varepsilon^{2}-\left[x-\left((3 a / 2)-d_{2}+\varepsilon\right) / 2\right]^{2}}, & q_{4}(x)=\frac{\varepsilon^{2}}{\varepsilon^{2}-\left[x-\left((3 a / 2)+d_{2}-\varepsilon\right) / 2\right]^{2}} \\
q_{5}(y)=\frac{\varepsilon^{2}}{\varepsilon^{2}-\left[y-\left((b / 2)-c_{1}+\varepsilon\right) / 2\right]^{2}}, & q_{6}(y)=\frac{\varepsilon^{2}}{\varepsilon^{2}-\left[y-\left((b / 2)+c_{1}-\varepsilon\right) / 2\right]^{2}}, \\
q_{7}(y)=\frac{\varepsilon^{2}}{\varepsilon^{2}-\left[y-\left((3 b / 2)-c_{2}+\varepsilon\right) / 2\right]^{2}}, & q_{8}(y)=\frac{c^{2}}{\varepsilon^{2}-\left[y-\left((3 b / 2)+c_{2}-\varepsilon\right) / 2\right]^{2}} .
\end{array}
$$


The elements of the matrix for the inhomogeneous geometry of the cross section of Figure 5a are calculated in Fourier space by

$$
\begin{aligned}
g(n, m)= & \frac{g_{0}}{a b}\left\{\int_{\left((a / 2)-d_{1}-\varepsilon\right) / 2}^{\left((a / 2)-d_{1}+\varepsilon\right) / 2} \exp \left(1-q_{1}(x)\right) \cos \left(\frac{n \pi x}{a}\right) d x\right. \\
& +\int_{\left((a / 2)-d_{1}+\varepsilon\right) / 2}^{\left((a / 2)+d_{1}-\varepsilon\right) / 2} \cos \left(\frac{n \pi x}{a}\right) d x+\int_{\left((a / 2)+d_{1}-\varepsilon\right) / 2}^{\left((a / 2)+d_{1}+\varepsilon\right) / 2} \exp \left(1-q_{2}(x)\right) \cos \left(\frac{n \pi x}{a}\right) d x \\
& +\int_{\left((3 a / 2)-d_{2}-\varepsilon\right) / 2}^{\left((3 a / 2)-d_{2}+\varepsilon\right) / 2} \exp \left(1-q_{3}(x)\right) \cos \left(\frac{n \pi x}{a}\right) d x \\
& \left.+\int_{\left((3 a / 2)-d_{2}+\varepsilon\right) / 2}^{\left((3 a / 2)+d_{2}-\varepsilon\right) / 2} \cos \left(\frac{n \pi x}{a}\right) d x+\int_{\left((3 a / 2)+d_{2}-\varepsilon\right) / 2}^{\left((3 a / 2)+d_{2}+\varepsilon\right) / 2} \exp \left(1-q_{4}(x)\right) \cos \left(\frac{m \pi y}{b}\right) d x\right\} \\
& \left\{\int_{\left((b / 2)-c_{1}-\varepsilon\right) / 2}^{\left((b / 2)-c_{1}+\varepsilon\right) / 2} \exp \left(1-q_{5}(y)\right) \cos \left(\frac{m \pi y}{b}\right) d y\right. \\
& +\int_{\left((b / 2)-c_{1}+\varepsilon\right) / 2}^{\left((b / 2)+c_{1}-\varepsilon\right) / 2} \cos \left(\frac{m \pi y}{b}\right) d y+\int_{\left((b / 2)+c_{1}-\varepsilon\right) / 2}^{\left((b / 2)+c_{1}+\varepsilon\right) / 2} \exp \left(1-q_{6}(y)\right) \cos \left(\frac{m \pi y}{b}\right) d y \\
& +\int_{\left((3 b / 2)-c_{2}-\varepsilon\right) / 2}^{\left((3 b / 2)-c_{2}+\varepsilon\right) / 2} \exp \left(1-q_{7}(y)\right) \cos \left(\frac{m \pi y}{b}\right) d y \\
& \left.+\int_{\left((3 b / 2)-c_{2}+\varepsilon\right) / 2}^{\left(\left((3 b / 2)+c_{2}-\varepsilon\right) / 2\right.} \cos \left(\frac{m \pi y}{b}\right) d y+\int_{\left((3 b / 2)+c_{2}-\varepsilon\right) / 2}^{\left((3 b / 2)+c_{2}+\varepsilon\right) / 2} \exp \left(1-q_{8}(y)\right) \cos \left(\frac{m \pi y}{b}\right) d y\right\} .
\end{aligned}
$$

According to Ref. [19]. The cyclic matrix $G$ is given by the form

$$
G=\left[\begin{array}{ccccccc}
g_{00} & g_{-10} & g_{-20} & \cdots & g_{-n m} & \cdots & g_{-N M} \\
g_{10} & g_{00} & g_{-10} & \cdots & g_{-(n-1) m} & \cdots & g_{-(N-1) M} \\
g_{20} & g_{10} & \ddots & \ddots & \ddots & & \\
\vdots & g_{20} & \ddots & \ddots & \ddots & & \\
g_{n m} & \ddots & \ddots & \ddots & g_{00} & \vdots & \\
\vdots & & & & & & \\
g_{N M} & \cdots & \cdots & \cdots & \cdots & \cdots & g_{00}
\end{array}\right]
$$

Similarly, the $\mathbf{G}_{\mathbf{x}}$ and $\mathbf{G}_{\mathrm{y}}$ matrices are obtained by the derivatives of the Fourier components of the dielectric profile. These matrices relate to the method that based on the Laplace and Fourier transforms, the inverse Laplace and Fourier transforms [19].

The proposed technique to calculate the elements of the matrix $\mathbf{G}$ relates to the method [19]. This method becomes an improved method by using the proposed technique also for discontinuous problems of the hollow rectangular waveguide.

\section{Numerical results}

This section presents several examples for the different geometries in the cross section. Five examples of the different discontinuous cross sections and complementary shapes are demonstrated in Figure 1a-e. All the next graphical results are 
Techniques for Calculating Two Interesting Types of Dielectric Materials in Straight Rectangular... DOI: http://dx.doi.org/10.5772/intechopen.88991

demonstrated as a response to a half-sine $\left(T E_{10}\right)$ input-wave profile and the inhomogeneous geometries of the cross section.

Figure $7 \mathbf{a}-\mathbf{e}$ shows the results of the output field as a response to a half-sine $\left(T E_{10}\right)$ input-wave profile for the rectangular dielectric profile (Figure $\mathbf{4 a}$ ) in the rectangular cross section for $\epsilon_{r}=3,5,7$, and 10, respectively, where $\mathrm{a}=\mathrm{b}=20 \mathrm{~mm}$, and $\mathrm{c}=\mathrm{d}=5 \mathrm{~mm}$. The center of the dielectric rectangle is located in the center of the cross section at the point $(\mathrm{a} / 2, \mathrm{~b} / 2)$, where $\epsilon_{r}=3,5,7$, and 10 , respectively. The output field in the same cross section of Figure 7a-d is shown in Figure 7e for the $\mathrm{x}$ axis where $\mathrm{y}=\mathrm{b} / 2=10 \mathrm{~mm}$, where $\epsilon_{r}=3,5,7$, and 10, respectively. The other parameters are $\mathrm{z}=15 \mathrm{~cm}, k_{0}=1671 / \mathrm{m}, \lambda=3.75 \mathrm{~cm}$, and $\beta=581 / \mathrm{m}$.

The results of Figure 7a-e are demonstrated for $\epsilon_{r}=3,5,7$, and 10, respectively. The results are strongly affected by the half-sine $\left(T E_{10}\right)$ input-wave profile, and the location of the center of the dielectric material.

The output field for the hollow rectangular waveguide where the dielectric material is located between the hollow rectangle and the metal is shown in

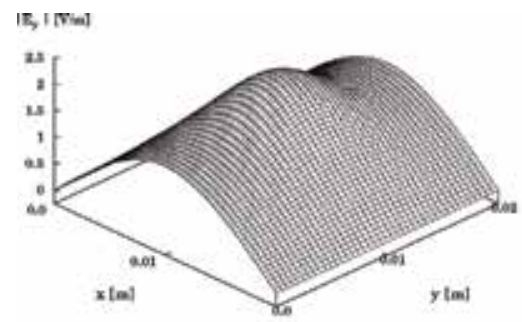

(a)

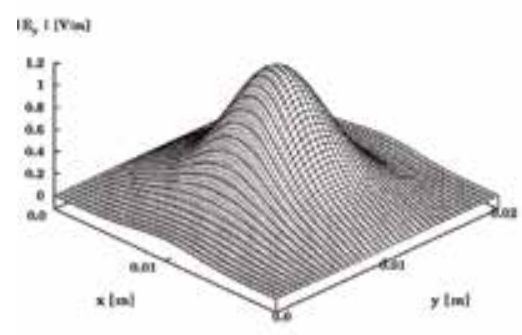

(c)

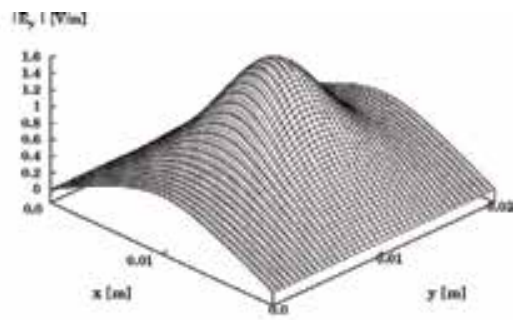

(b)

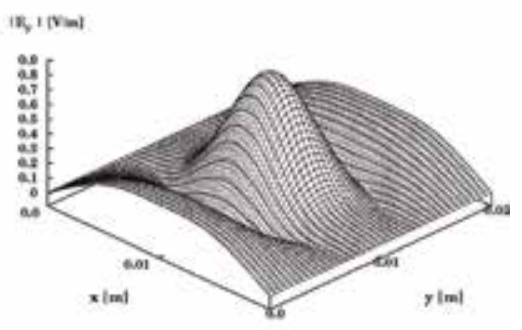

(d)

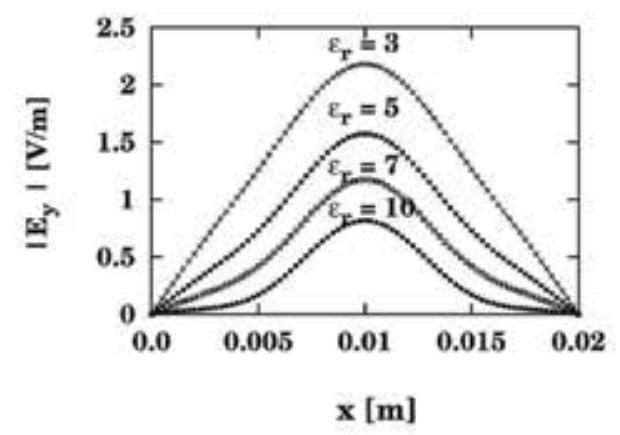

(e)

Figure 7.

The output field as a response to a half-sine $\left(T E_{10}\right)$ input-wave profile for the rectangular dielectric profile

Figure $4 a$ in the rectangular cross section, where $a=b=20 \mathrm{~mm}$, and $c=d=5 \mathrm{~mm}$. The center of the rectangle dielectric profile is located in the center of the cross section, for $(a) \epsilon_{r}=3$, for $(b) \epsilon_{r}=5$, for $(c) \epsilon_{r}=7$, and for $(d) \epsilon_{r}=10$. (e). The output field in the same cross section of the results $(a-d)$ for $x$-axis and where $y=b / 2=10 \mathrm{~mm}$, for the values of $\epsilon_{r}=3,5,7$, and 10 , respectively. 
Figure 8a-d, where $\mathrm{a}=\mathrm{b}=20 \mathrm{~mm}$, and $\mathrm{c}=\mathrm{d}=14 \mathrm{~mm}$. In this case the thickness of the dielectric layer is equal to $3 \mathrm{~mm}(\mathrm{e}=\mathrm{f}=3 \mathrm{~mm})$. The results are demonstrated for $\epsilon_{r}=2.5, \epsilon_{r}=3, \epsilon_{r}=3.5$, and $\epsilon_{r}=4$, respectively. The output field in the same cross section of Figure 8a-d is shown in Figure 8e, for $\varepsilon_{r}=2.5,3,3.5$, and 4, respectively, for $\mathrm{x}$-axis where $\mathrm{y}=\mathrm{b} / 2=10 \mathrm{~mm}$. In this case, $\mathrm{z}=15 \mathrm{~cm}, k_{0}=1671 / \mathrm{m}, \lambda=3.75 \mathrm{~cm}$, and $\beta=581 / \mathrm{m}$. These results are shown for $\epsilon_{r}=2.5,3,3.5$, and 4 , respectively, and these results are strongly affected by the half-sine $\left(T E_{10}\right)$ input-wave profile, the location of the center of the dielectric material, and the thickness of the dielectric material between the hollow rectangle and the metal.

We can find the relevant parameters to obtain the Gaussian behavior of the output field. We can show that the Gaussian behavior is obtained when the thickness of the dielectric layer is equal to $3 \mathrm{~mm}$, as shown in the results of Figure 8a-d. The output results are strongly affected by the thickness of the dielectric layer.

Figure 9a-d shows the results of the output field as a response to a half-sine $\left(T E_{10}\right)$ input-wave profile for the cross section with four dielectric profiles, as

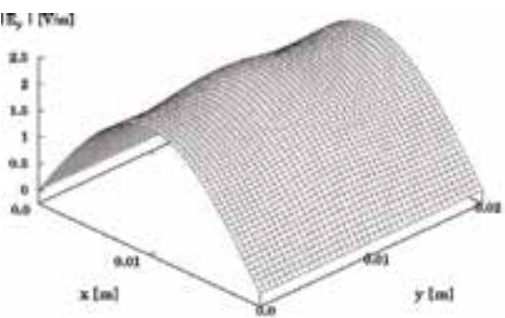

(a)

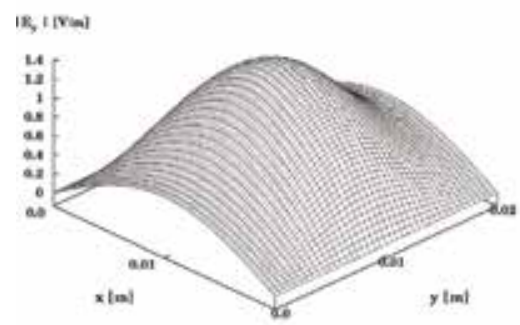

(c)

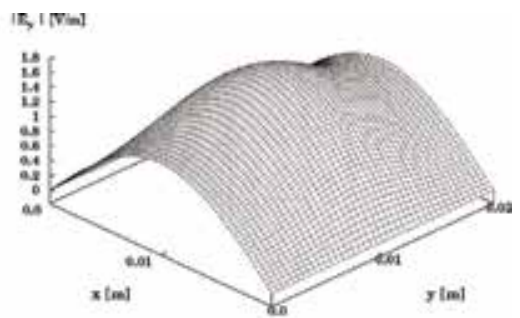

(b)

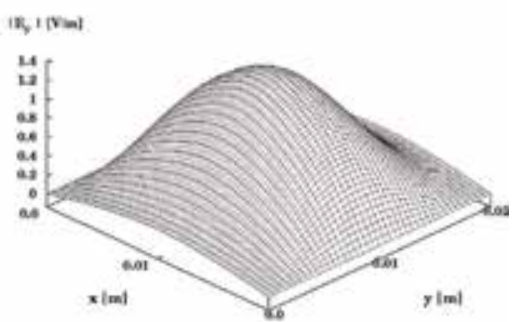

(d)

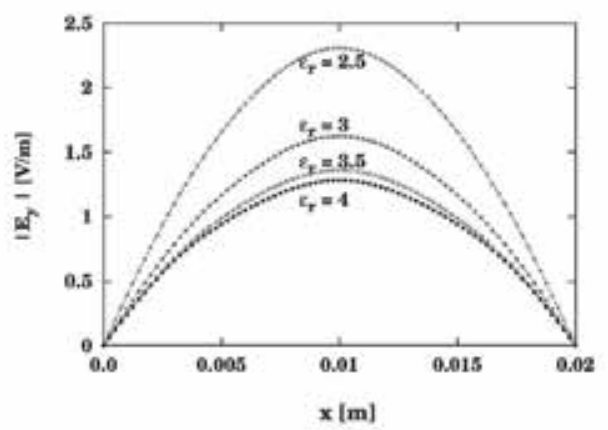

(e)

Figure 8.

The output field as a response to a half-sine $\left(T E_{10}\right)$ input-wave profile for the hollow rectangular waveguide where the hollow rectangle is located in the center of the cross section Figure $4 b$, where $a=b=20 \mathrm{~mm}$, $c=d=14 \mathrm{~mm}$. The thickness of the dielectric layer is equal to $3 \mathrm{~mm}(e=f=3 \mathrm{~mm})$. The results are shown for (a) $\epsilon_{r}=2.5$, for, (b) $\epsilon_{r}=3$, for, (c) $\epsilon_{r}=3.5$, and for, $(d) \epsilon_{r}=4$, (e) the output field in the same cross section of the results $(a-d)$ is shown for $x$-axis and where $y=b / 2=10 \mathrm{~mm}$, for $\epsilon_{r}=2.5,3,3.5$, and 4, respectively. 


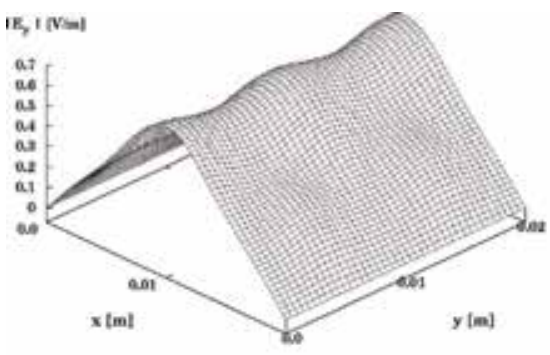

(a)

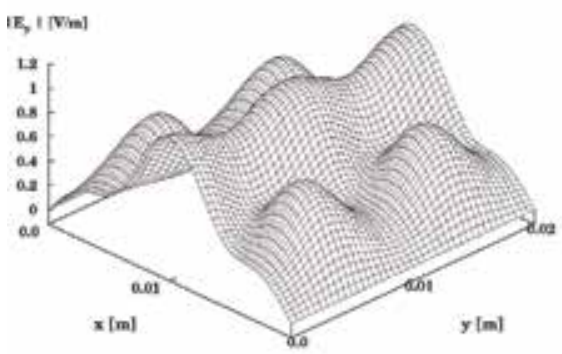

(c)

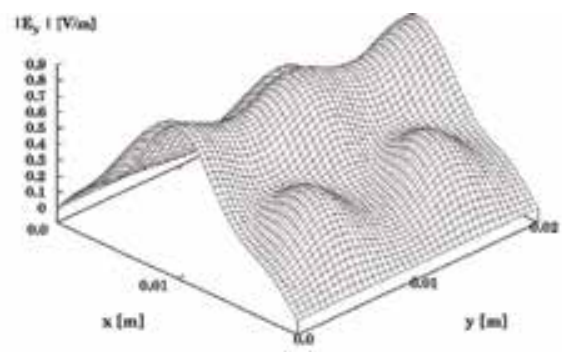

(b)

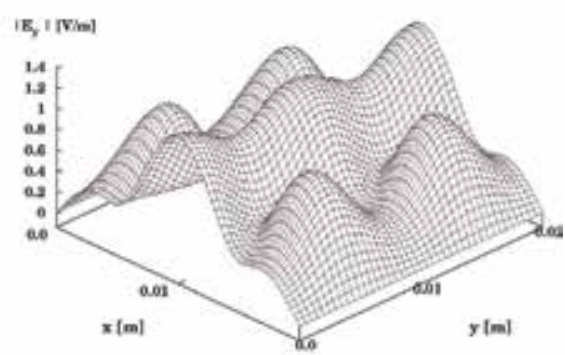

(d)

Figure 9.

The output field as a response to a half-sine $\left(T E_{10}\right)$ input-wave profile for the cross section with four dielectric profiles, as shown in Figure 5 a. The centers of the first, the second, the third and the firth dielectric rectangles are located at the points $(a / 4, b / 4),(3 a / 4, b / 4),(a / 4,3 b / 4)$, and $(3 a / 4,3 b / 4)$, respectively. The parameters are $a=b=20 \mathrm{~mm}, c=d=0.4 \mathrm{~mm}$, and $z=15 \mathrm{~cm}$, where $(a) . \epsilon_{r}=1.1 ;(b) . \epsilon_{r}=1.2$; (c). $\epsilon_{r}=1.3 ;(d) . \epsilon_{r}=1.4$.

shown in Figure 5a. In this example we suppose that $a=b=20 \mathrm{~mm}, c=d=0.4 \mathrm{~mm}$, $\mathrm{z}=15 \mathrm{~cm}$, the centers of the first, the second, the third, and the firth dielectric rectangles are located at the points $(a / 4, b / 4),(3 a / 4, b / 4),(a / 4,3 b / 4)$, and $(3 a / 4$, $3 \mathrm{~b} / 4)$, respectively, and for $\varepsilon_{r}=1.1,1.2,1.3$ and 1.4 , respectively. The other parameters are $k_{0}=1671 / \mathrm{m}, \lambda=3.75 \mathrm{~cm}$ and $\beta=581 / \mathrm{m}$. By increasing the parameter $\epsilon_{r}$ from 1.1 to 1.4 , the output dielectric profile increased, the output profile of the halfsine $\left(T E_{10}\right)$ profile decreased, and the output amplitude increased. These results are strongly affected by the half-sine $\left(T E_{10}\right)$ input-wave profile, and the locations of the rectangular profiles along $\mathrm{x}$-axis and $\mathrm{y}$-axis.

Figure 10a-d shows the results of the output field as a response to a half-sine $\left(T E_{10}\right)$ input-wave for the hollow waveguide with four hollow rectangles, where $\mathrm{a}=\mathrm{b}=20 \mathrm{~mm}, \mathrm{c}=\mathrm{d}=3.3 \mathrm{~mm}$, and $\mathrm{z}=15 \mathrm{~cm}, \epsilon_{r}=1.2,1.3,1.4$, and 1.5 , respectively. The centers of the first, the second, the third and the firth hollow rectangles are located at the points $(\mathrm{a} / 4, \mathrm{~b} / 4),(3 \mathrm{a} / 4, \mathrm{~b} / 4),(\mathrm{a} / 4,3 \mathrm{~b} / 4)$, and $(3 \mathrm{a} / 4,3 \mathrm{~b} / 4)$, respectively. The other parameters are $k_{0}=1671 / \mathrm{m}, \lambda=3.75 \mathrm{~cm}$ and $\beta=581 / \mathrm{m}$. These results are strongly affected by the half-sine $\left(T E_{10}\right)$ input-wave profile, and the locations of the rectangular profiles along $\mathrm{x}$-axis and $\mathrm{y}$-axis.

By increasing only the parameter $\epsilon_{r}$, the output dielectric profile increased, the output profile of the half-sine $\left(T E_{10}\right)$ profile decreased, and the output amplitude increased. These results are strongly affected by the half-sine $\left(T E_{10}\right)$ input-wave profile, and the locations of the rectangular profiles along $\mathrm{x}$-axis and $\mathrm{y}$-axis.

The applications are useful for straight waveguides in millimeter regimes, in the cases where the dielectric profile is located in the center of the cross section, for the cases where the hollow rectangle is located in the center of the cross section, and also for complicated and discontinuous profiles in the cross section. 


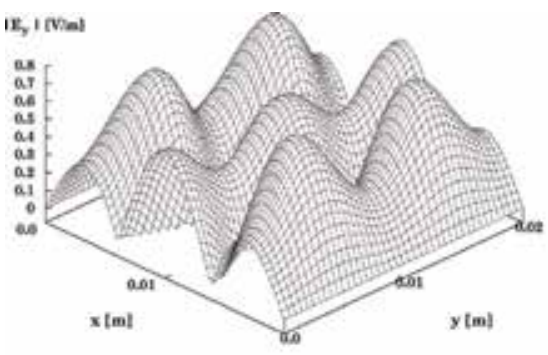

(a)

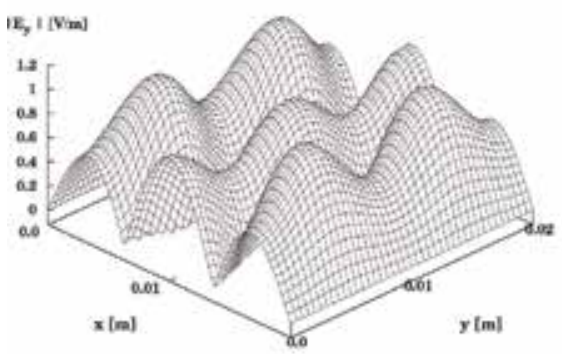

(c)

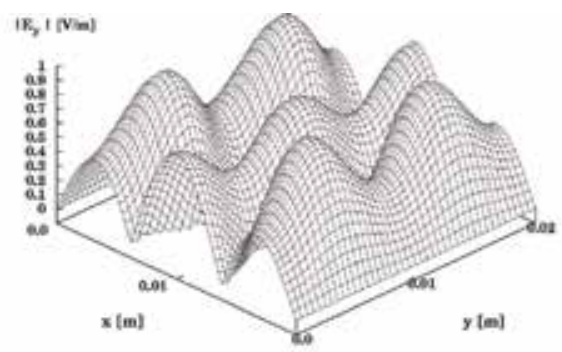

(b)

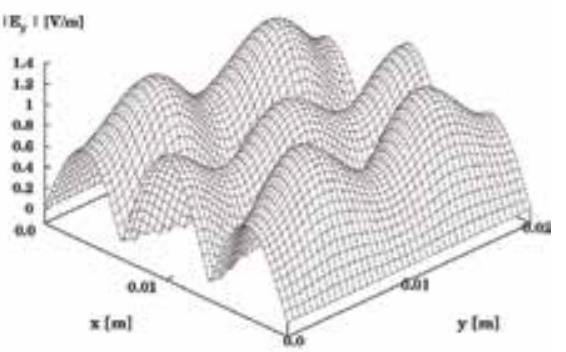

(d)

Figure 10.

The output field as a response to a half-sine $\left(T E_{10}\right)$ input-wave profile for the hollow waveguide with four hollow rectangles, as shown in Figure $5 \boldsymbol{b}$. The centers of the first, the second, the third and the firth hollow rectangles are located at the points $(a / 4, b / 4),(3 a / 4, b / 4),(a / 4,3 b / 4)$, and $(3 a / 4,3 b / 4)$, respectively. The parameters are $a=b=20 \mathrm{~mm}, c=d=3.3 \mathrm{~mm}$, and $z=15 \mathrm{~cm}$, where hollow waveguide with four hollow rectangles, as shown in Figure 1e, (a). $\epsilon_{r}=1.5 ;(b) . \epsilon_{r}=2 ;(c) . \epsilon_{r}=2.5 ;(d) . \epsilon_{r}=3$.

\section{Conclusions}

This chapter presents two interesting types of dielectric materials in the straight rectangular waveguides and their applications. Five examples of the different discontinuous cross section were demonstrated. The effective technique was proposed for all case of Figure 1a-e, in order to calculate the dielectric material in the specific cross section.

The proposed techniques are very important to understand the influence of the dielectric materials and the hollow rectangles in all case of discontinuous geometry in the cross section. All the graphical results are demonstrated as a response to a halfsine $\left(T E_{10}\right)$ input-wave profile. The proposed techniques relate to the method based on Laplace and Fourier transforms and the inverse Laplace and Fourier transforms.

The method is based on Fourier transform, thus we need use with the image method to calculate the dielectric profile in the cross section. The image method and periodic replication are needed for fulfilling the boundary condition of the metallic waveguide.

Figure 8a-e relates to the hollow rectangular waveguide where the hollow rectangle is located in the center of the cross section as shown in Figure $\mathbf{4 b}$. We can find the relevant parameters to obtain the Gaussian behavior of the output field. From the results of Figure 8a-e, the Gaussian behavior is obtained when the thickness of the dielectric layer is equal to $3 \mathrm{~mm}$. The output results are strongly affected by the thickness of the dielectric layer.

By increasing only the parameter $\epsilon_{r}$, the output dielectric profile increased, the output profile of the half-sine $\left(T E_{10}\right)$ profile decreased, and the output amplitude 
Techniques for Calculating Two Interesting Types of Dielectric Materials in Straight Rectangular... DOI: http://dx.doi.org/10.5772/intechopen.88991

increased. The results are strongly affected by the half-sine $\left(T E_{10}\right)$ input-wave profile, the location of the center of the dielectric material, and the thickness of the dielectric material between the hollow rectangle and the metal.

The applications are useful for straight waveguides in millimeter regimes, in the cases where the dielectric profile is located in the center of the cross section, for cases where the hollow rectangle is located in the center of the cross section, and also for complicated and discontinuous profiles in the cross section.

\section{Author details}

Zion Menachem

Department of Electrical Engineering, Sami Shamoon College of Engineering, Beer Sheva, Israel

*Address all correspondence to: zionme@sce.ac.il; zionmm@gmail.com

\section{IntechOpen}

(C) 2019 The Author(s). Licensee IntechOpen. This chapter is distributed under the terms of the Creative Commons Attribution License (http://creativecommons.org/licenses/ by/3.0), which permits unrestricted use, distribution, and reproduction in any medium, provided the original work is properly cited. (c) BY 


\section{References}

[1] Chiang KS. Review of numerical and approximate methods for the modal analysis of general optical dielectric waveguides. Optical and Quantum Electronics. 1994;26:S113-S134

[2] Saad M. Review of numerical methods for the analysis of arbitrarilyshaped microwave and optical dielectric waveguides. IEEE Transactions on Microwave Theory and Techniques. 1985;MTT-33:894-899

[3] Yeap KH, Teh KH, Yeong KC, Lai KC, Loh MC. Propagation in dielectric rectangular waveguides. Optica Applicata. 2016;XLVI:317-330

[4] Bartling JQ. Propagation of an electromagnetic wave in an infinite rectangular dielectric wave guide. Journal of the Franklin Institute. 1969; 287:389-407

[5] Khorasani S. Differential transfermatrix method for solution of onedimensional linear nonhomogeneous optical structures. Optical Society of America. 2003;20:91-96

[6] Rojas JAM, Alpuente J, Pineiro J, Sanchez R. Rigorous full vectorial analysis of electromagnetic wave propagation in 1D inhomogeneous media. Progress in Electromagnetics Research. 2006;63:89-105

[7] Mehrany K, Khorasani S. Analytical solution of non-homogeneous anisotropic wave equations based on differential transfer matrices. Journal of Optics A. 2002;4:624-635

[8] Lee JF. Finite element analysis of lossy dielectric waveguides. IEEE Transactions on Microwave Theory and Techniques. 1994;42:1025-1031

[9] Ise K, Inoue K, Koshiba M. Threedimensional finite-element method with edge elements for electromagnetic waveguide discontinuities. IEEE Transactions on Microwave Theory and Techniques. 1991;39:1289-1295

[10] Singh V, Joshi M, Prasad B, Ojha P. Modal dispersion characteristics and waveguide dispersion of an optical waveguide having a new unconventional core cross-section. Journal of Electromagnetic Waves and Applications. 2004;18:455-468

[11] Hernandez-Lopez MA, Quintillan M. Propagation characteristics of modes in some rectangular waveguides using the finitedifference time-domain method. Journal of Electromagnetic Waves and Applications. 2000;14:1707-1722

[12] Mallick AK, Sanyal GS. Electromagnetic wave propagation in a rectangular waveguide with sinusoidally varying width. IEEE Transactions on Microwave Theory and Techniques. 1978;MTT-26:243-249

[13] Bogle A, Havrilla M, Nyquis D, Kempel L, Rothwell E. Electromagnetic material characterization using a partially-filled rectangular waveguide. Journal of Electromagnetic Waves and Applications. 2005;19:1291-1306

[14] Vaish A, Parthasarathy H. Analysis of a rectangular waveguide using finite element method. Progress in Electromagnetics Research C. 2008;2: 117-125

[15] Khalaj-Amirhosseini M. Analysis of longitudinally inhomogeneous waveguides using the method of moments. Progress In Electromagnetics Research. 2007;74:57-67

[16] Khalaj-Amirhosseini M. Analysis of nonuniform transmission lines using Taylor's series expansion. International 
Techniques for Calculating Two Interesting Types of Dielectric Materials in Straight Rectangular... DOI: http://dx.doi.org/10.5772/intechopen.88991

Journal of RF and Microwave

Computer-Aided Engineering. 2006;10: 536-544

[17] Molina-Fernandez I, Camacho-

Penalosa C, Ramos JI. Application of the two-dimensional Fourier transform to nonlinear wave propagation phenomena. IEEE Transactions on Microwave Theory and Techniques. 1994;42:1079-1085

[18] Henry CH, Verbeek BH. Solution of the scalar wave equation for arbitrarily shaped dielectric waveguides by twodimensional Fourier analysis. Journal of Lightwave Technology. 1989;7:308-313

[19] Menachem Z, Jerby E. Transfer matrix function (TMF) for propagation in dielectric waveguides with arbitrary transverse profiles. IEEE Transactions on Microwave Theory and Techniques. 1998;46:975-982

[20] Vladimirov V. Equations of Mathematical Physics. New York, NY: Marcel Dekker; 1971 



\title{
Research Progress on Synergistic Effect between Insulation Gas Mixtures
}

\author{
Su Zhao and Dengming Xiao
}

\begin{abstract}
Synergetic effect is a special gas discharge phenomenon among insulating gas mixtures, which has important reference value for gas selection of future gasinsulated power equipment. The research progress and investigation methods of synergistic effect and insulation characteristics of different gas mixtures at home and abroad are reviewed in this chapter. The synergistic effect between different kinds of gas mixtures including $\mathrm{SF}_{6}$ gas mixtures and some new insulation gases such as $\mathrm{c}-\mathrm{C}_{4} \mathrm{~F}_{8}, \mathrm{CF}_{3} \mathrm{I}$, and $\mathrm{C}_{4} \mathrm{~F}_{7} \mathrm{~N}$ is presented. Combined with the results of multiple studies, it can be seen that the synergistic effect of the gas mixture has a certain relationship with the electronic transport parameters and discharge patterns. Besides, the synergistic effect of the same gas mixture may change with the change of external conditions such as gas pressure, voltage type, and electrode distance.
\end{abstract}

Keywords: gas discharge, synergistic effect, gas mixture, insulation, environmental friendly gas

\section{Introduction}

With the continuous development of power industry, gas-insulated power equipment has been used more and more widely in electrical transmission system. Gas-insulated power equipment mainly includes gas-insulated transformer (GIT), gas-insulated transmission line (GIL), and gas-insulated switchgear (GIS). These gasinsulated equipment are noncombustible, nonexplosive, safe, and stable and have long maintenance period [1] . At present, $\mathrm{SF}_{6}$ gas is the most widely used gas in power equipment, which has excellent insulation performance and arc extinguishing ability. As early as the $1940 \mathrm{~s}, \mathrm{SF}_{6}$ gas has been used as insulation gas in power equipment [2]. Since the 1970s, researchers have studied the use of gas mixture including $\mathrm{SF}_{6}$ and some buffer gases such as $\mathrm{N}_{2}, \mathrm{CO}_{2}, \mathrm{Ar}, \mathrm{He}$, air, etc. to replace pure $\mathrm{SF}_{6}$ as the insulation medium [3-5]. With further study, researchers found that the insulation characteristics of gas mixtures containing $\mathrm{SF}_{6}$ gas did not increase linearly according to the mixing ratio of $\mathrm{SF}_{6}$, and the electrical strength of the mixture was higher than the weighted average of the electrical strength of the two gas components. In 1980, Wootton and Chantry first used synergism to describe this phenomenon [6]. In the study of synergistic effect, scholars have found that there are also super synergistic effect and negative synergistic effect, which are shown in Figure 1.

Suppose that the composition of gas mixture is gas 1 and gas 2, the breakdown voltage of gas 1 is $U_{1}$, the breakdown voltage of gas 2 is $U_{2}$, and $U_{1}>U_{2}$, the 


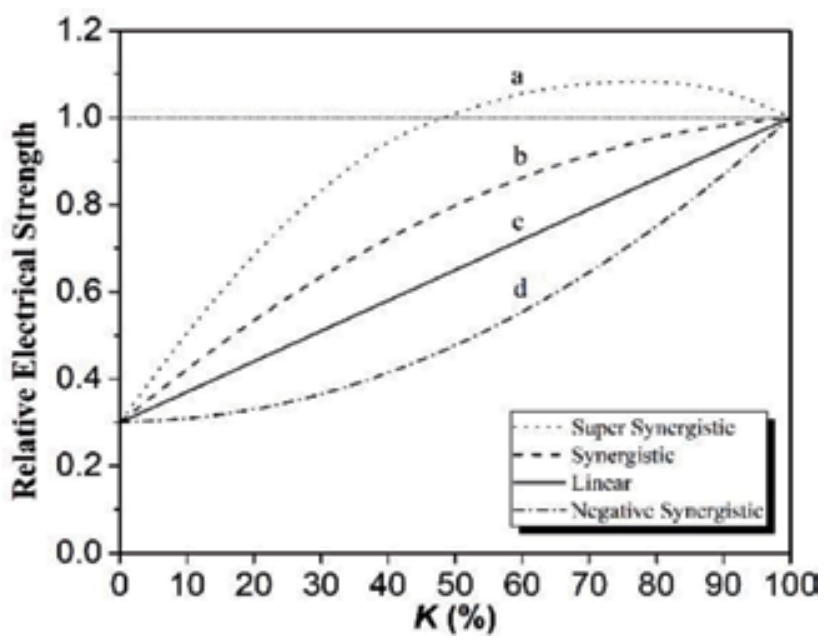

Figure 1.

Types of synergistic effect.

breakdown voltage of gas mixture is $U_{\mathrm{m}}$, and $K$ is the mixing ratio of gas 1 in the gas mixture. For super synergistic effect, $U_{\mathrm{m}}>U_{1}$ and $U_{\mathrm{m}}>U_{2}$ exist in some mixing ratios, as shown in curve a; for synergistic effect, $U_{\mathrm{m}}>U_{1}+U_{2}$, as shown in curve b; for linear relationship, $U_{\mathrm{m}}=U_{1}+U_{2}$, as shown in curve c; for negative synergistic effect, $U_{\mathrm{m}}<U_{1}+U_{2}$, as shown in curve d in Figure 1 .

At first, researchers studied the synergistic effect of $\mathrm{SF}_{6}$ gas mixture in order to solve the problems of high liquefaction temperature, sensitivity to electric field, and the high price of $\mathrm{SF}_{6}$ gas [7]. However, with the deepening understanding of $\mathrm{SF}_{6}$, scholars have found that $\mathrm{SF}_{6}$ is a strong greenhouse gas. It is estimated that the global annual production of $\mathrm{SF}_{6}$ gas is more than 20,000 tons and $80 \%$ of $\mathrm{SF}_{6}$ gas produced globally each year is used in the power industry. Although $\mathrm{SF}_{6}$ has many advantages, its greenhouse effect on the earth cannot be ignored. The global warming potential (GWP) value of $\mathrm{SF}_{6}$ gas is 23,900; it means that the emission of $1 \mathrm{~kg} \mathrm{SF}_{6}$ is equivalent to the emission of $23,900 \mathrm{~kg}$ of $\mathrm{CO}_{2}$. What is more serious is that $\mathrm{SF}_{6}$ has a very stable chemical property, which is difficult to decompose after it spreads to the outside environment, and can exist for up to 3200 years. The environmental impact and greenhouse effect generated by $\mathrm{SF}_{6}$ will continue to accumulate [8-10].

Affected by climate change, more and more international cooperation has been carried out to reduce greenhouse gas emissions, so as to curb global climate change and maintain the sustainable development of the environment. In the Kyoto protocol of the United Nations framework convention on climate change signed in Kyoto, Japan, in $1997, \mathrm{SF}_{6}$ has been clearly regulated as one of the six greenhouse gases and requires developed countries to freeze and reduce the total greenhouse gas emissions [11]. It means that the use of $\mathrm{SF}_{6}$ in the industrial field will be increasingly restricted and pressured. Therefore, it is an urgent task to study a new gas insulation scheme to replace $\mathrm{SF}_{6}$.

Although at present there have been many studies about alternative $\mathrm{SF}_{6}$ gas, no gas can thoroughly replace $\mathrm{SF}_{6}$ gas in the form of a single gas; they all have to be mixed with buffer gas for industrial application. The reasonable use of synergistic effect can effectively improve dielectric strength of gas mixtures and reduce the use of insulation gas, so in this paper, the research progress and methods of synergistic effect with gas mixtures are introduced; the prospect and the difficulties in the field were also discussed. This paper is expected to provide help and reference for future research on synergistic effect. 


\section{Synergistic effect in $\mathrm{SF}_{6}$ gas mixtures}

In view of the synergistic effect and insulation strength of $\mathrm{SF}_{6}$ mixture, scholars studied and analyzed it through theoretical calculation and experimental research. Figure 2a is a simple and intuitive calculation method proposed by Wieland et al. for the insulation strength of $\mathrm{SF}_{6}$ gas mixture, and Figure $\mathbf{2 b}$ is the comparison of the calculation results with the actual values and weighted values [12].

Christophorou et al. thought that the preferred gas mixture should include effective electron-attaching gas and/or electron-slowing down gas [13]. The attachment cross section of electron-attaching components should be as wide as possible, or the attachment cross section of different gases is in different energy interval, so the attachment cross section of gas mixtures is also wide. The effect of electron-slowing down gas is to slow down the free electrons, making them easier to attach and reducing secondary ionization. Based on this theory, they believed that when an electronegative gas and a gas with a large dipole moment are mixed, the synergistic effect and insulation strength of the gas mixture will be better. Figure 3 shows the experimental results of $\mathrm{SF}_{6}$ gas mixtures with $\mathrm{CF}_{4}, \mathrm{CHF}_{3}$, and 1,1,1- $\mathrm{CH}_{3} \mathrm{CF}_{3}$ gas, and the electric dipole moments of these three gases are $0,1.65 \mathrm{D}$, and 2.32D, respectively.

From the figure, it can be seen that the breakdown voltage curve of $\mathrm{SF}_{6}-\mathrm{CF}_{4}$ gas mixtures shows almost a linear trend, and the electric dipole moments of $\mathrm{CF}_{4}$ is 0 . When it comes to $\mathrm{SF}_{6}-\mathrm{CHF}_{3}$ gas mixture, as the content of $\mathrm{SF}_{6}$ gas increases, the breakdown voltage of the gas mixtures does not increase in a straight line, and there is synergistic effect that occurs. The electric dipole moments of $1,1,1-\mathrm{CH}_{3} \mathrm{CF}_{3}$ gas is $2.32 \mathrm{D}$, which is the one with the largest electric dipole moment among the three gases; from Figure 3c it can be seen that the synergistic effect of this gas mixture is the most pronounced.

Okubo et al. investigated the partial discharge (PD) and breakdown characteristics of $\mathrm{SF}_{6}-\mathrm{N}_{2}$ gas mixtures in order to analyze the relationship between electronegativity, additive gases, and the insulation strength [14]. They believed that the synergistic effect of gas mixture is related to the change of discharge form. Figure 4 shows the

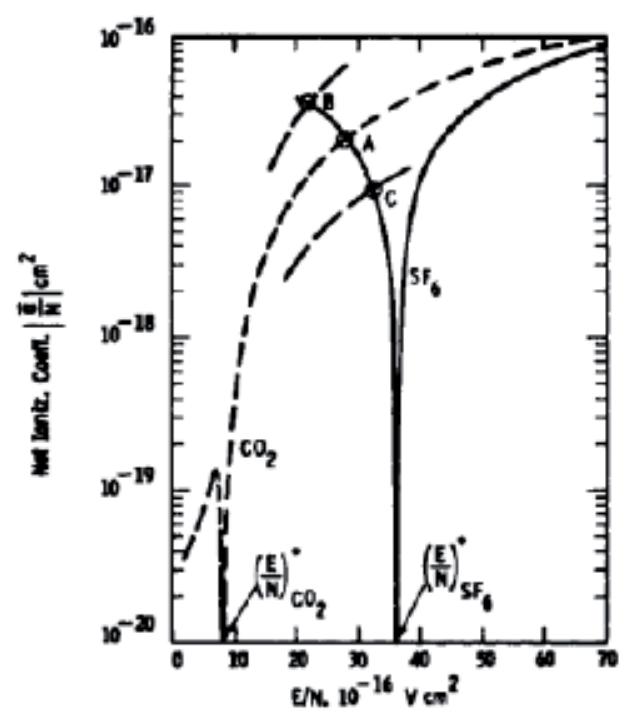

(a)

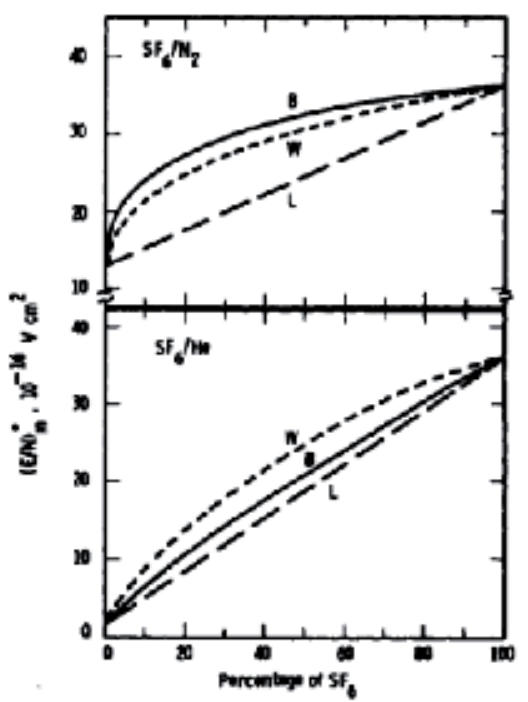

(b)

Figure 2.

Experimental results of $\mathrm{SF}_{6}$ gas mixtures. (a) Wieland calculation method, (b) Results comparison of different methods. 


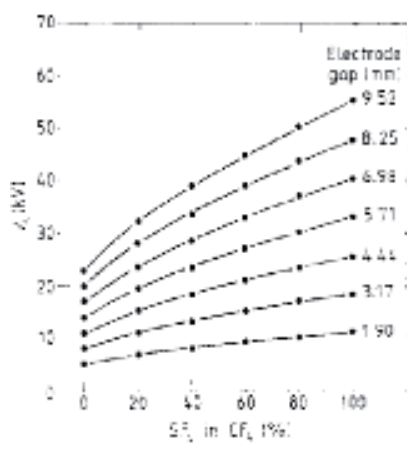

(a)

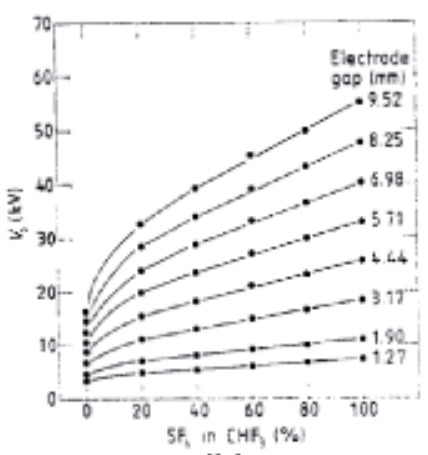

(b)

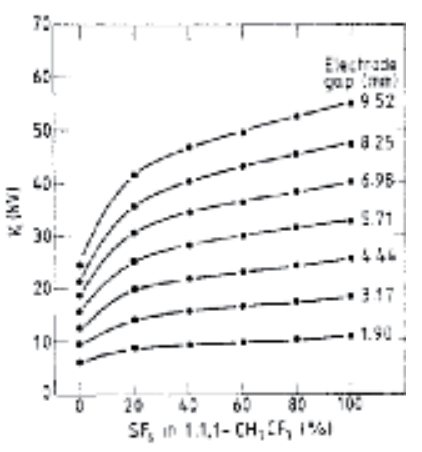

(c)

Figure 3.

Experimental results of $\mathrm{SF}_{6}$ gas mixtures. (a) $\mathrm{SF}_{6}-\mathrm{CF}_{4}$, (b) $\mathrm{SF}_{6}-\mathrm{CHF}_{3}$, (c) $\mathrm{SF}_{6}-1,1,1-\mathrm{CH}_{3} \mathrm{CF}_{3}$.

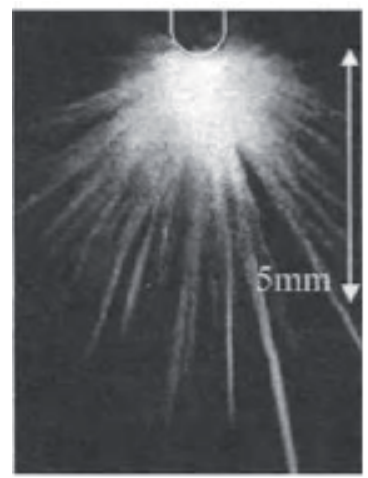

(a)

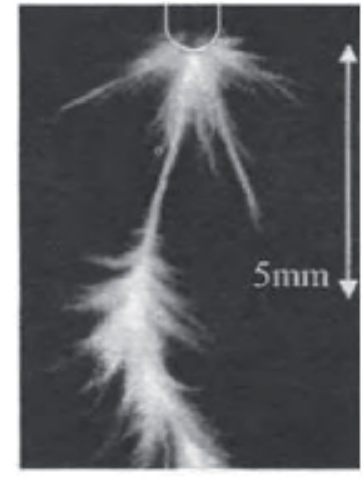

(b)

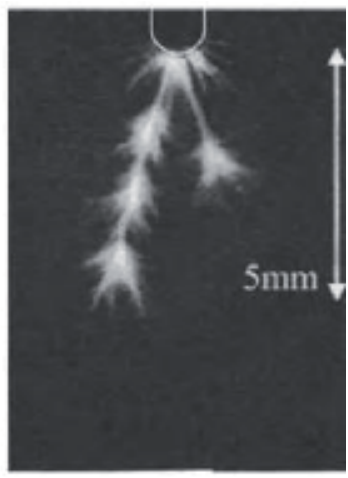

(c)

Figure 4.

Impulse PD in $\mathrm{SF}_{6}-\mathrm{N}_{2}$ gas mixture. (a) o.1 $\mathrm{MPa},(\mathrm{b}) 0.2 \mathrm{MPa}$, (c) o.3 $\mathrm{MPa}$.

impulse $\mathrm{PD}$ of $\mathrm{SF}_{6}-\mathrm{N}_{2}$ gas mixture at different pressure. The needle-plate electrode was used in the experiment, and the content of $\mathrm{SF}_{6}$ was $10 \%$ and $\mathrm{N}_{2}$ was $90 \%$.

It can be seen that with the change of gas pressure, the development of impulse PD has changed. When the gas pressure is $0.1 \mathrm{MPa}$, as shown in Figure 5a, the brushlike partial discharge occurred around the needle electrode, and it can be thought of as streamer discharge. When the pressure increases to $0.2 \mathrm{MPa}$, it can be seen from Figure $5 \mathrm{~b}$ that the development of discharge process becomes longer; streamer discharge turns into leader discharge. As the pressure continues to increase, when the pressure is $0.3 \mathrm{MPa}$, the PD type of the gas mixture is still the leader discharge, and the path of discharge development becomes shorter with the increase of the pressure.

Yamada et al. studied the insulation properties of a kind of gas mixture containing ultra-dilute $\mathrm{SF}_{6}$ gas [15]. It has been found that trace $\mathrm{SF}_{6}$ has a significant effect on the streamer discharge of the gas mixture. As the $\mathrm{SF}_{6}$ content increases, the number of the discharge channels decreased significantly, and the number of channels that can reach the plane electrode also reduced, as shown in Figure 4. Except for the effect on discharge characteristics, the results show that the breakdown voltage and the $\mathrm{PD}$ voltage of $\mathrm{SF}_{6} / \mathrm{N}_{2}$ gas mixture have a significant synergistic effect. Yamada $\mathrm{T}$ thought that the addition of trace $\mathrm{SF}_{6}$ inhibits the development of streamer discharge process, which leads to synergistic effect.

Osmokrovic et al. conducted an in-depth study on the synergistic effect of $\mathrm{SF}_{6} / \mathrm{N}_{2}$ under impulse voltage. The experimental results show that the synergistic 


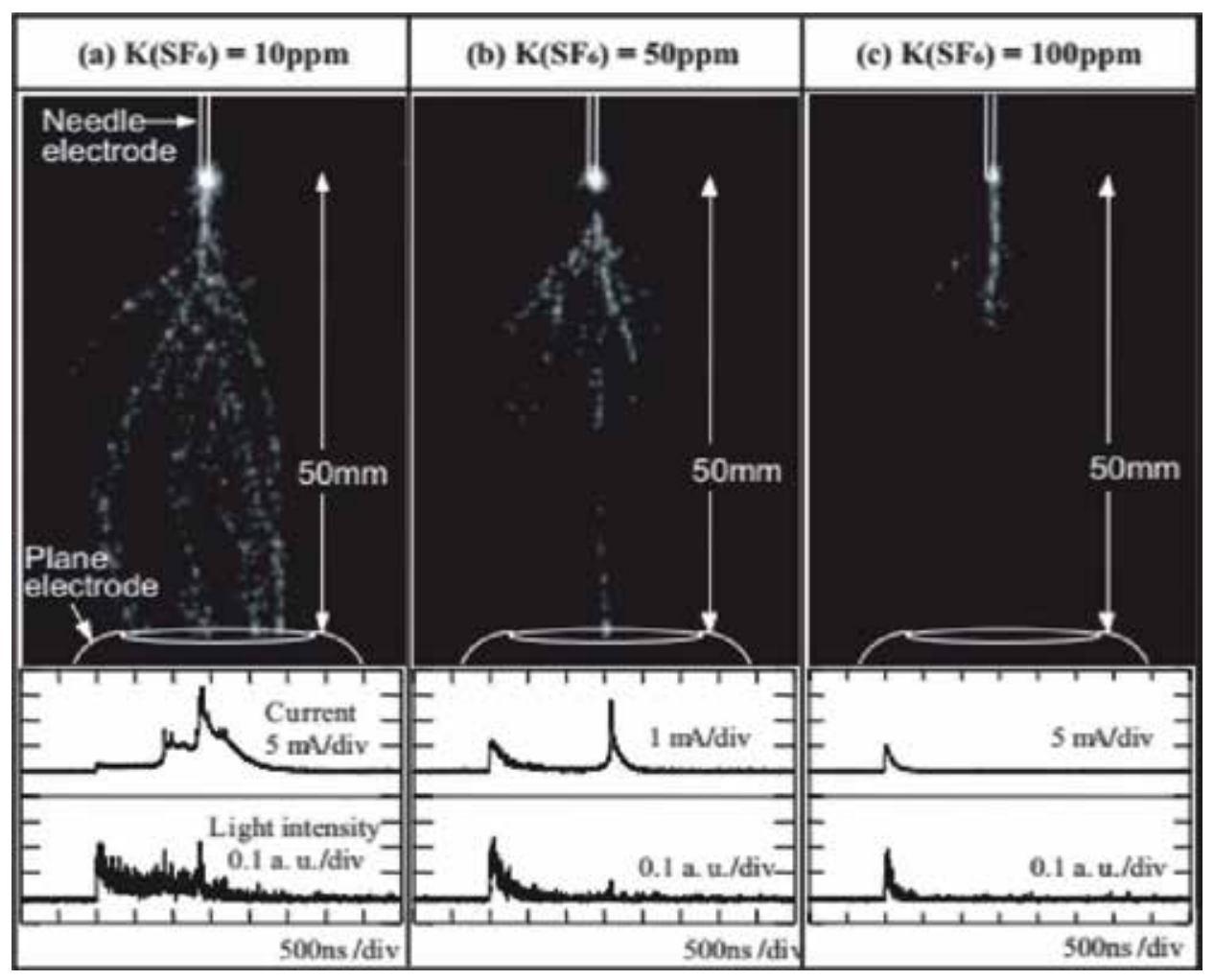

Figure 5.

$P D$ characteristics in $\mathrm{SF}_{6}-\mathrm{N}_{2}$ gas mixture.

effect of $\mathrm{SF}_{6} / \mathrm{N}_{2}$ gas mixture related to the rising rate of the impulse voltage. As the impulse voltage rise rate increases, the synergistic effect is gradually weakened. The synergistic effect of $\mathrm{SF}_{6}$ gas mixture is very weak and almost completely disappears under some impulse voltage with very high rise rate [16]. The insulation characteristics of $\mathrm{SF}_{6}$ and $\mathrm{SF}_{6} / \mathrm{N}_{2}$ gas mixture under impulse voltage with different rise rate are shown in Figure 5. The rise rates of shock voltage in Figure 6a-c are 1, 50 and $800 \mathrm{kv} / \mathrm{ms}$, respectively.

Based on the above phenomenon, Osmokrovic proposed that after adding $\mathrm{N}_{2}$ to the $\mathrm{SF}_{6}$ gas, electrons can make $\mathrm{N}_{2}$ vibration and rotation dynamics excited or dissociated, this process will make electrons lose energy, and the effective temperature decreases, to realize the modulation of electron energy spectrum and increase the probability that the electrons are captured. The rise rate of impulse voltage has influence on the modulation of the electron energy spectrum, which in turn affects the synergistic effect of $\mathrm{SF}_{6}$ gas mixture.

Hayakawa et al. studied the generation and development of PD characteristics of $\mathrm{SF}_{6} / \mathrm{N}_{2}$ gas mixture under positive lightning impulse [17]. PD and breakdown characteristics with different $\mathrm{SF}_{6}$ content are shown in Figure 7. Hayakawa proved through the film of the streak camera and ICCD that the discharge process in $\mathrm{SF}_{6} /$ $\mathrm{N}_{2}$ gas mixture did change with the increase of gas pressure. Streamer discharge and leader discharge are the two types of $\mathrm{PD}$ process in $\mathrm{SF}_{6} / \mathrm{N}_{2}$ gas mixture, which all have relationship with gas pressure and $\mathrm{SF}_{6}$ content. With the increase of gas pressure and the content of $\mathrm{SF}_{6}$ gas, the leader discharge process gradually takes the leading position, and the streamer discharge process of gradually weakens.

Chen studied the discharge characteristics of $\mathrm{SF}_{6} / \mathrm{N}_{2}$ gas mixture under DC voltage and lightning impulse in extremely uneven electric field. The experimental 


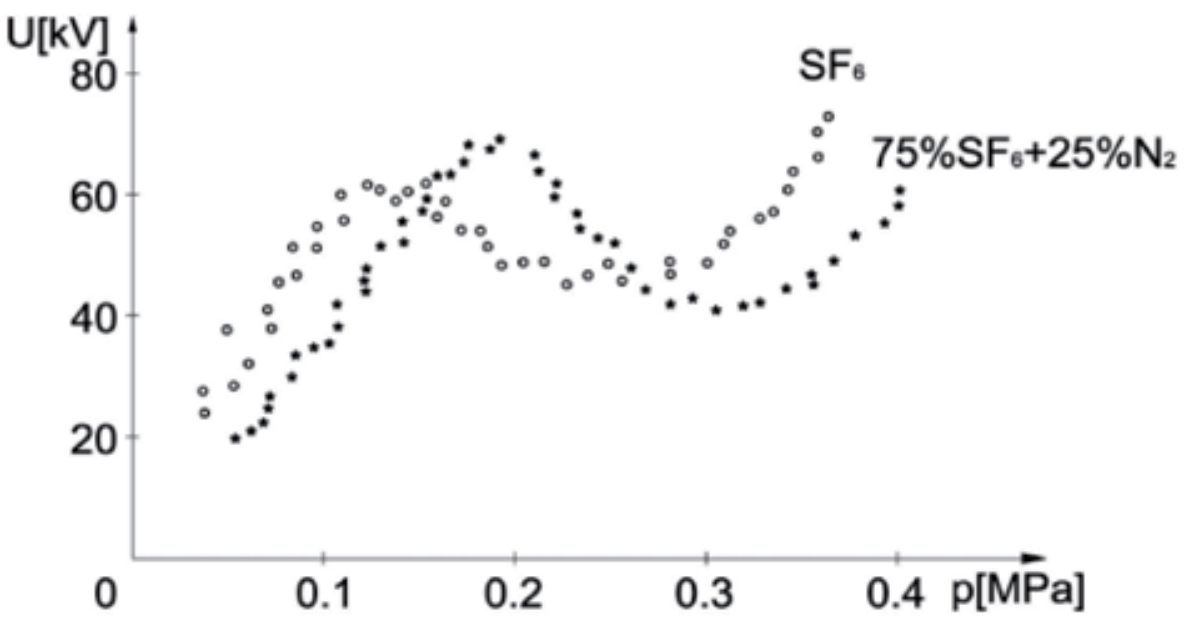

(a)
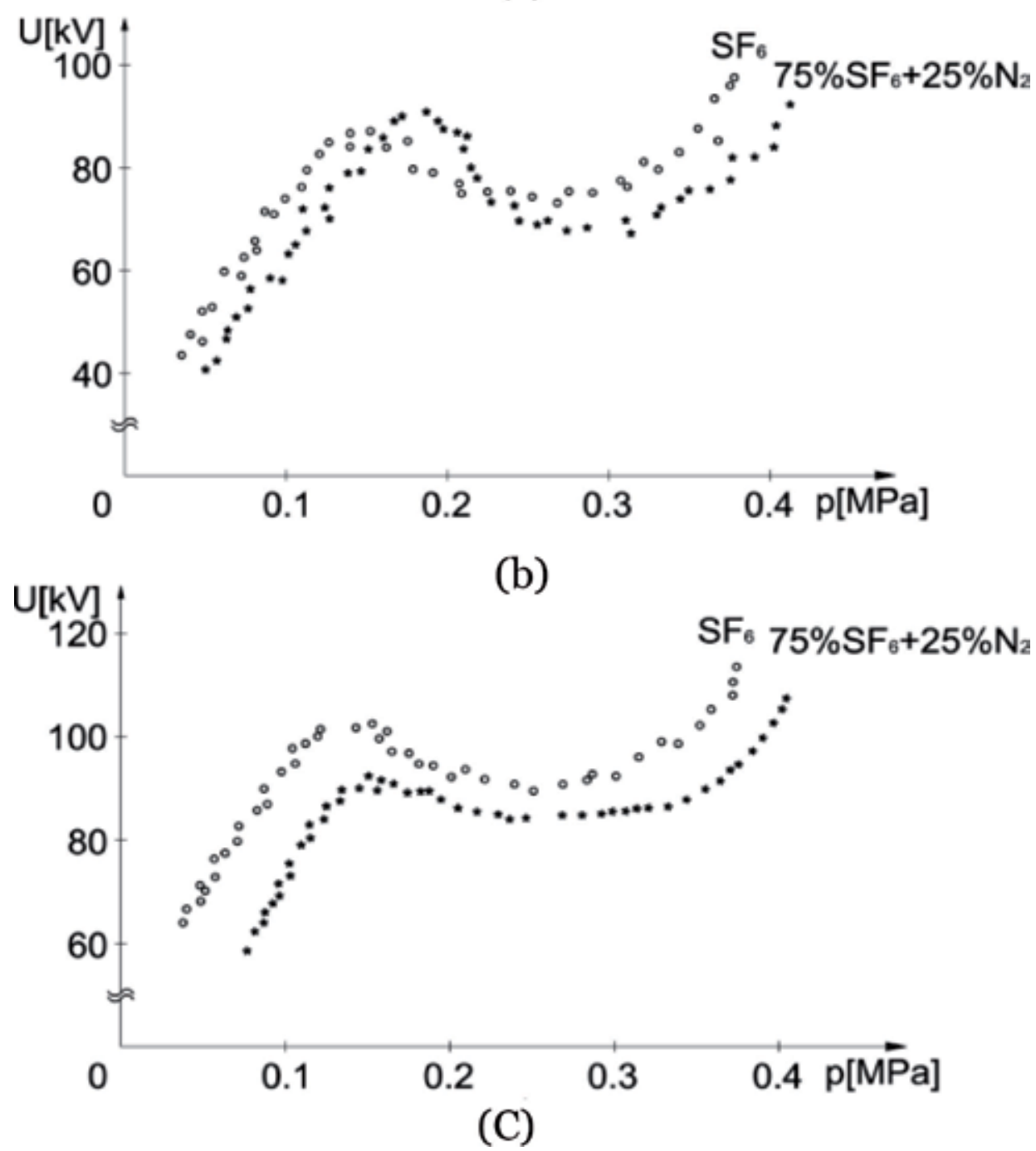

Figure 6.

Insulation characteristics of $\mathrm{SF}_{6}$ and $\mathrm{SF}_{6} / \mathrm{N}_{2}$ gas mixture under different rising rate impulse voltages. (a) $1 \mathrm{kV} / \mathrm{ms}$, (b) $50 \mathrm{kv} / \mathrm{ms}$, (C) $800 \mathrm{kV} / \mathrm{ms}$. 


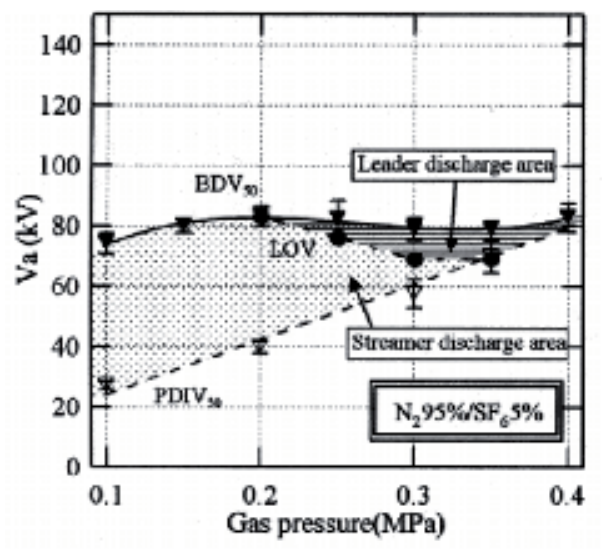

(a)

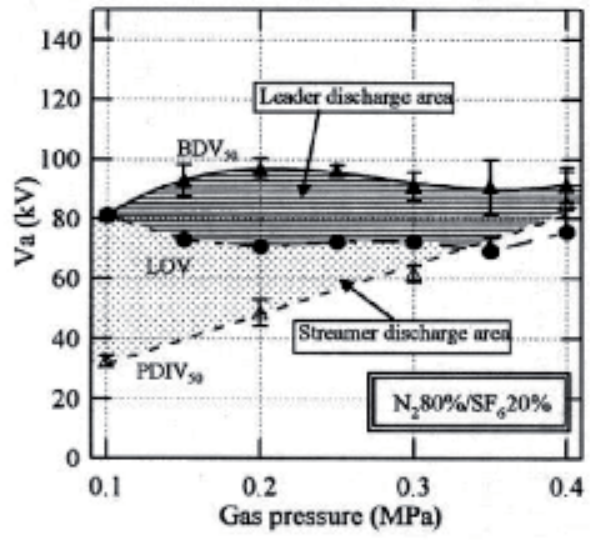

(c)

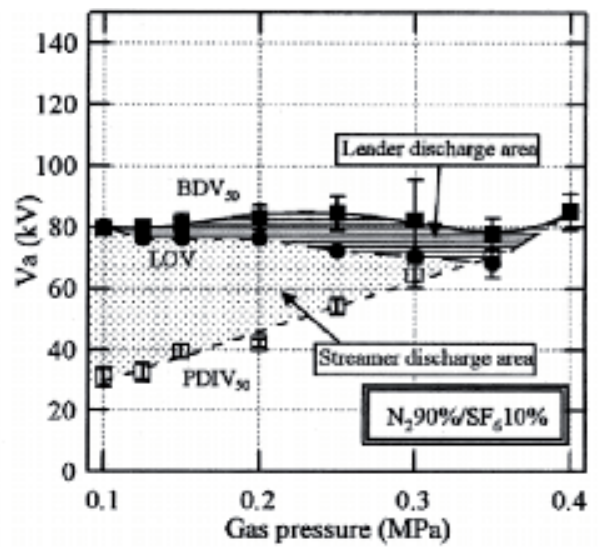

(b)

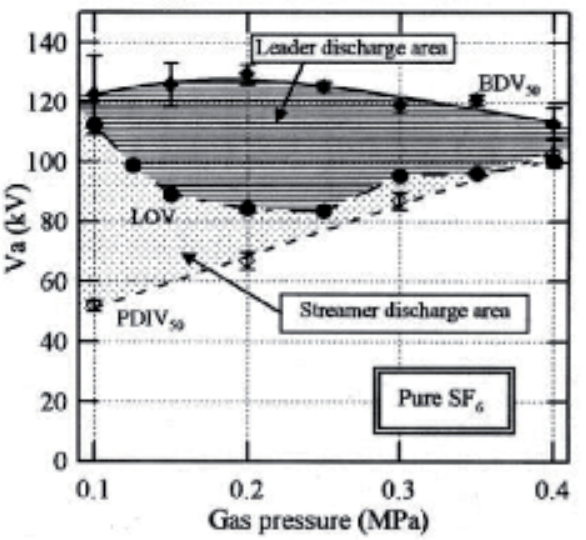

(d)

Figure 7 .

$P D$ and breakdown characteristics with different $S F_{6}$ content. $\left(B D V_{50}\right.$, breakdown voltage; $P D I V_{50}, 50 \%$ probability $P D$ inception voltage; $L O V$, leader discharge onset voltage). (a) $S F_{65}-N_{2} 95 \%$, (b) $S F_{6} 10-N_{2} 90 \%$, (c) $\mathrm{SF}_{6} \mathrm{OO}-\mathrm{N}_{2} 80 \%$, (d) pure $S F_{6}$.

results show that there was a significant decrease of breakdown voltage with the increase of gas pressure under DC voltage and the abnormal discharge characteristics pressure range of $\mathrm{SF}_{6} / \mathrm{N}_{2}$ gas mixture is larger than $\mathrm{SF}_{6}$ gas. This phenomenon leads to the breakdown voltage of $\mathrm{SF}_{6} / \mathrm{N}_{2}$ gas mixture is higher than that of $\mathrm{SF}_{6}$ gas. So under this abnormal range, positive synergistic effect occurs. However, under lighting impulse voltage, no abnormal discharge phenomenon was found, but positive synergistic effect still existed, as shown in Figure 8 [18].

Tagashira et al. believe that synergistic effects can be divided into three categories: $a$-synergistic effect $\left(\mathrm{SF}_{6}+\mathrm{SiH}_{4}\right), \eta$-synergistic effect $\left(\mathrm{SF}_{6}+\mathrm{c}-\mathrm{C}_{4} \mathrm{~F}_{8}\right)$, and $\gamma$-synergistic effect $\left(\mathrm{N}_{2}+\mathrm{CH}_{4}\right)$. The research found that the curves of $\mathrm{SF}_{6} / \mathrm{SiH}_{4}$, $\mathrm{SF}_{6} / \mathrm{c}-\mathrm{C}_{4} \mathrm{~F}_{8}, \mathrm{SF}_{6} / \mathrm{C}_{3} \mathrm{~F}_{6}$ gas mixtures with $\mathrm{SF}_{6}$ gas content all decreased first and then increased, that is, the curve had a minimum point. For the curve of $\mathrm{SF}_{6} / \mathrm{SiH}_{4}$ gas mixture, the falling part of the curve is due to the decrease of ionization coefficient $\alpha$, that is, $\alpha$-synergistic effect, and for the curve of $\mathrm{SF}_{6} / \mathrm{c}-\mathrm{C}_{4} \mathrm{~F}_{8}$ gas mixture, the rising part is caused by the increase of attachment coefficient $\eta$ and that is $\eta$-synergistic effect. In addition to these two synergistic effects, they also proposed a synergistic effect of $\gamma$ on the secondary ionization coefficient of $\mathrm{N}_{2}+\mathrm{CH}_{4}$ gas mixture [19]. 


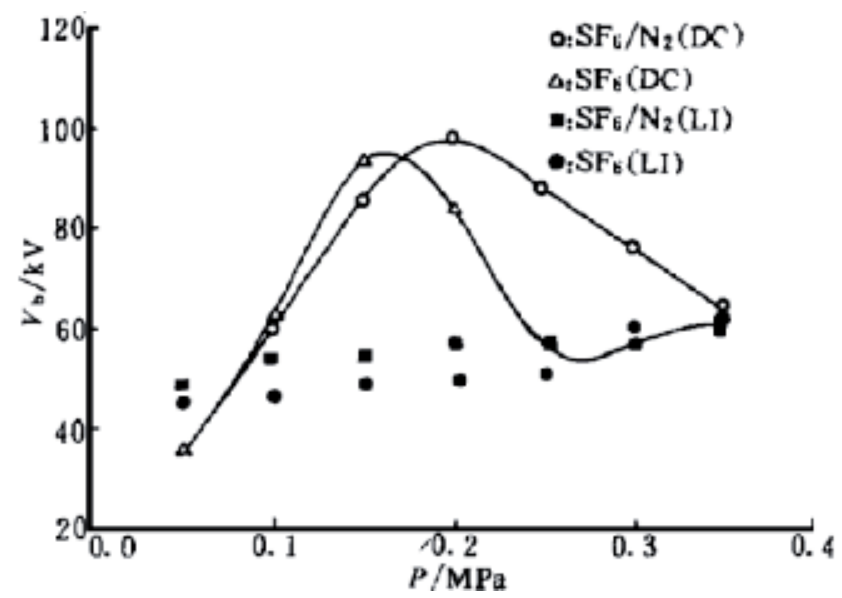

Figure 8.

Abnormal discharge phenomenon in $\mathrm{SF}_{6} / \mathrm{N}_{2}$ and $\mathrm{SF}_{6}$.

Takuma et al. studied the synergistic effect of gas mixtures such as $\mathrm{SF}_{6} / \mathrm{N}_{2}$, $\mathrm{CCl}_{2} \mathrm{~F}_{2} / \mathrm{N}_{2}$, etc. They assumed that the effective ionization coefficient of the gas mixture is equal to the sum of the coefficients of the two component gases multiplied by their respective partial pressure ratios, suggesting an empirical formula for breakdown voltage of $\mathrm{SF}_{6} / \mathrm{N}_{2}$ gas mixture under slight uneven electric field [20]:

$$
U_{m}=U_{2}+\frac{k}{k+C(1-k)}\left(U_{1}-U_{2}\right)
$$

where $U_{1}$ and $U_{2}$ are the breakdown voltage of component gas 1 and gas $2, U_{\mathrm{m}}$ is the breakdown voltage of gas mixture $\left(U_{1}>U_{2}\right), k$ is the partial pressure ratio of component gas 1 , and $C$ is the synergistic effect coefficient, which is independent of the partial pressure ratio $k$. When Constant $C=0.08$, the calculated and experimental values of the $\mathrm{SF}_{6} / \mathrm{N}_{2}$ gas mixture by this formula are shown in Figure 5 . The breakdown voltage values are basically the same, which can reflect the synergistic effect of $\mathrm{SF}_{6}$ gas mixture.

According to formula (1), the formula for calculating the synergy coefficient proposed by Takuma is

$$
C=\frac{k\left(U_{1}-U_{M}\right)}{(1-k)\left(U_{M}-U_{2}\right)}
$$

As can be seen from Eq. (2), when $C=1$, the breakdown voltage of the gas mixture is equal to the weighting value of breakdown voltage of the two components according to the mixing ratio. That is, the breakdown voltage of the gas mixture exhibits a linear relationship that increases as the mixing ratio of the component gas increases. When $0<C<1$, the breakdown voltage of the gas mixture reflects synergistic effect phenomenon, and the smaller the value of $C$, the more significant the nonlinear increase of the breakdown voltage of the gas mixture, which means synergistic effect becomes more significant. When $C=0$, the breakdown voltage of the gas mixture equals to the breakdown voltage of gas 1 , which means $U_{\mathrm{M}}=U_{1}$.

If all the situations of synergistic effect, i.e., positive synergistic effect, synergistic effect, and negative synergistic, are considered at the same time, then the above formula is no longer applicable. Assuming a positive synergistic effect of the gas mixture, it can be seen from the calculation that the coefficient $C<0$ under positive synergistic. When the breakdown voltage of the gas mixture is lower than all the breakdown voltage of the component gas, that is, the phenomenon of "super 
negative synergistic effect" appears, then value of the coefficient $C$ is still less than 0 , so the various synergistic effects of the gas mixture cannot be clearly distinguished by formula (2).

Guo et al. introduced a normalization coefficient $h$ to investigate the synergistic effect of $\mathrm{SF}_{6} / \mathrm{N}_{2}$ gas mixture under lightning impulse. The definition of coefficient $\mathrm{h}$ is as follows:

$$
\begin{array}{ll}
h=\frac{\left(U_{m}-U_{2}\right)-k\left(U_{1}-U_{2}\right)}{0.5\left(U_{1}+U_{2}\right)} & 0<k<1 \\
h=0 & k=0 \text { or } 1
\end{array}
$$

where $U_{1}, U_{2}, U_{\mathrm{m}}$, and $k$ in Eq. 3 have the same meaning as those in Eq. 2. When $h>0$, it means that gas mixture has synergistic effect, and when $h<0$, it represents that the gas mixture has negative synergistic effect. The relationship between the

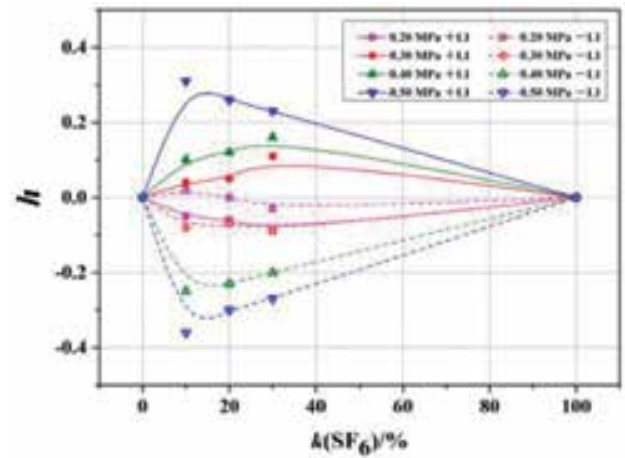

(a)

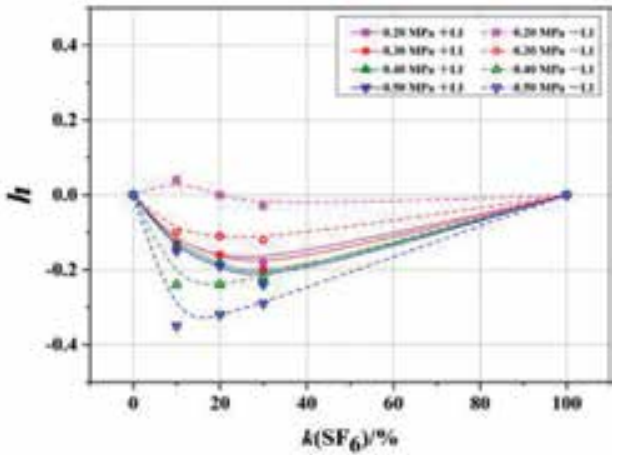

(b)

Figure 9.

Coefficient $\mathrm{h}$ for $\mathrm{SF}_{6} / \mathrm{N}_{2}$ gas mixture under lightning impulse with different needle-plane electrode ( $\mathrm{R}$ is radius curvatures of needle electrodes). (a) $R=2 \mathrm{~mm},(b) R=1 \mathrm{~mm}$.
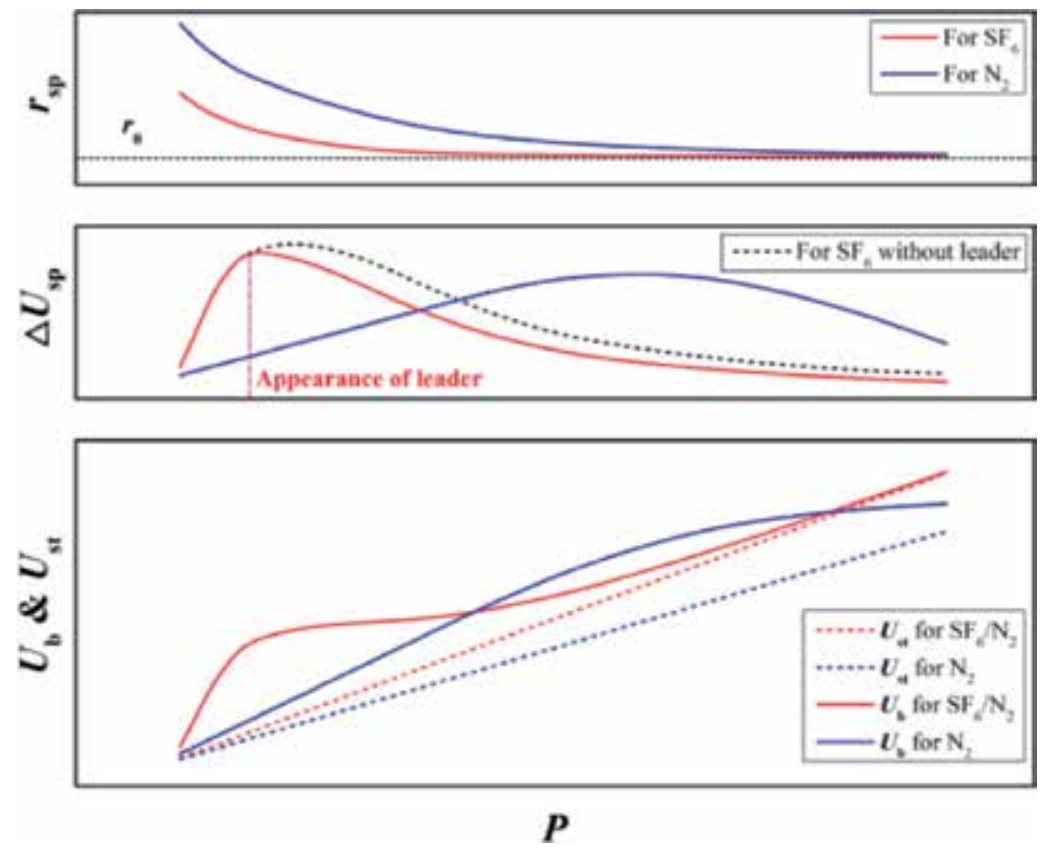

Figure 10.

Breakdown voltage differences between $N_{2}$ and $S_{6} / N_{2}$. 
coefficient $h$ of $\mathrm{SF}_{6} / \mathrm{N}_{2}$ and $k$ under lightning impulse with different needle-plane electrodes is shown in Figure 9 [21].

The analysis results show that under the action of negative lightning impulse voltage, the negative synergistic effect increases with the increase of gas pressure. The synergistic effect under the positive impact voltage decreases with the decrease of gas pressure. With the increase of the electric field inhomogeneity coefficient, the synergistic effect has a negative synergistic effect. The analysis of the development process of the flow discharge shows that there are three reasons for the negative synergistic effect: similar flow corona starting voltage, different space charge effects, and different $\mathrm{N}_{2}$ and $\mathrm{SF}_{6} / \mathrm{N}_{2}$ mixed gas discharge forms. The difference of breakdown voltage between $\mathrm{N}_{2}$ and $\mathrm{SF}_{6} / \mathrm{N}_{2}$ gas mixture is shown in Figure 10 . $r_{\mathrm{sp}}$ represents the range of space charge, $\Delta U_{\mathrm{SP}}$ is the influence of space charge on breakdown voltage, $U_{\mathrm{st}}$ is the streamer corona onset voltage, and $U_{\mathrm{b}}=U_{\mathrm{st}}+\Delta U_{\mathrm{SP}}$.

\section{Synergistic effect in $\mathrm{SF}_{6}$ alternative gas mixtures}

\section{$3.1 \mathrm{c}-\mathrm{C}_{4} \mathrm{~F}_{8}$}

c- $\mathrm{C}_{4} \mathrm{~F}_{8}$ is colorless, odorless, nonflammable, and nonexplosive; the GWP of c- $\mathrm{C}_{4} \mathrm{~F}_{8}$ is about 8700 , and the molecular structure of $\mathrm{c}-\mathrm{C}_{4} \mathrm{~F}_{8}$ is shown in Figure 11. The insulation performance of pure $\mathrm{c}_{-} \mathrm{C}_{4} \mathrm{~F}_{8}$ gas is better than $\mathrm{SF}_{6}$, and the gas mixture of $\mathrm{c}-\mathrm{C}_{4} \mathrm{~F}_{8}$ gas has similar insulation characteristics to $\mathrm{SF}_{6}$, which can meet the requirements of practical application. The liquefaction temperature of $c-\mathrm{C}_{4} \mathrm{~F}_{8}$ is about $-6^{\circ} \mathrm{C}$, higher than that of $\mathrm{SF}_{6}$, which is $-63.8^{\circ} \mathrm{C}[22]$. Therefore, $c-\mathrm{C}_{4} \mathrm{~F}_{8}$ can only be mixed with the gas in a certain proportion to reduce the overall liquefaction temperature of the gas mixture for application.

While studying the synergistic effect of $\mathrm{SF}_{6}$ gas mixture, Christophorou et al. also conducted a comparative study on the synergistic effect of $\mathrm{c}-\mathrm{C}_{4} \mathrm{~F}_{8}$ gas mixtures.

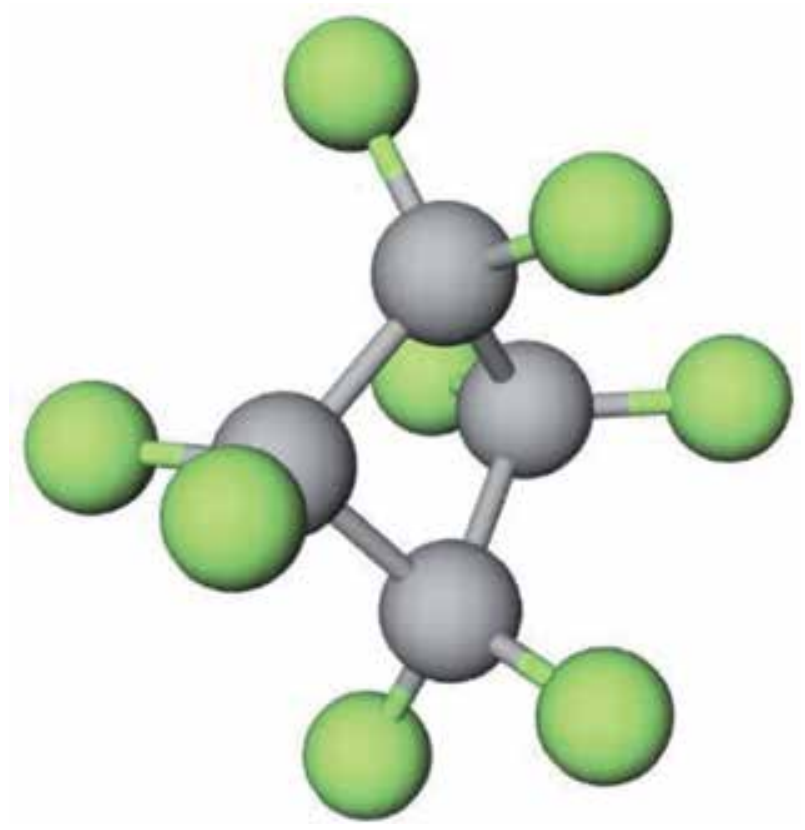

Figure 11.

Molecular structure of $c-C_{4} F_{8}$. 


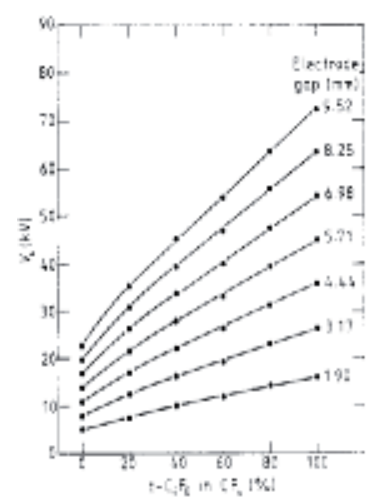

(a)

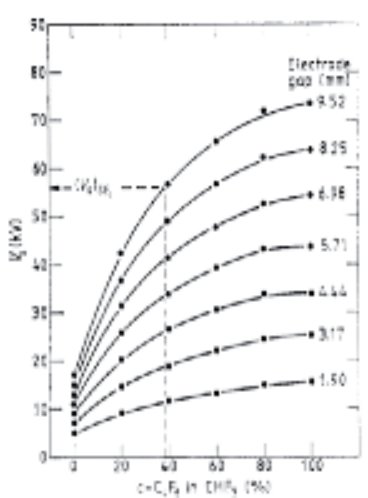

(b)

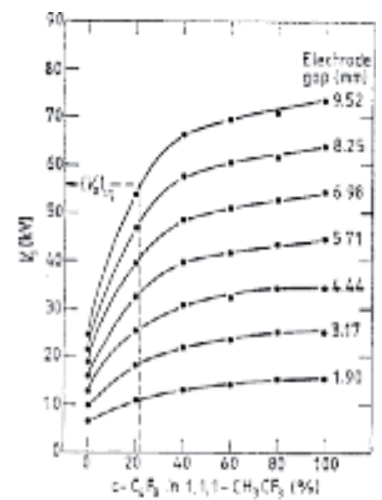

(c)

Figure 12.

Breakdown voltage of $c-C_{4} F_{8}$ gas mixtures for various electrode gaps. (a) $c-C_{4} F_{8} / C F_{4},(b) c-C_{4} F_{8} / C H F_{3},(c)$ $c-\mathrm{C}_{4} \mathrm{~F}_{8} / 1,1,1-\mathrm{CH}_{3} \mathrm{CF}_{3}$.

The insulation characteristics and synergistic effect of $\mathrm{c}-\mathrm{C}_{4} \mathrm{~F}_{8} / \mathrm{CF}_{4}$ and $\mathrm{c}-\mathrm{C}_{4} \mathrm{~F}_{8} / \mathrm{CHF}_{3}$ gas mixture are shown in Figure 12 [13].

It can be seen from the figure that the addition of a small amount of $\mathrm{c}-\mathrm{C}_{4} \mathrm{~F}_{8}$ can greatly improve the insulation strength of gas mixture. Mixing $20 \% \mathrm{c}-\mathrm{C}_{4} \mathrm{~F}_{8}$ in $\mathrm{CHF}_{3}$ can double the breakdown voltage. The insulation strength of $\mathrm{c}-\mathrm{C}_{4} \mathrm{~F}_{8} / 1,1,1-\mathrm{CH}_{3} \mathrm{CF}_{3}$ gas with the same mixing ratio is the same as that of pure $\mathrm{SF}_{6}$ gas. By comparison, it can be seen that the synergistic effect phenomenon will also occur when $c-\mathrm{C}_{4} \mathrm{~F}_{8}$ gas and the gas with large dipole moment are mixed.

Xing et al. discussed the feasibility of replacing $\mathrm{SF}_{6}$ gas with $\mathrm{c}-\mathrm{C}_{4} \mathrm{~F}_{8} / \mathrm{N}_{2}$ mixture for gas insulation equipment from the perspective of $\mathrm{PD}$ performance. The initial $\mathrm{PD}$ voltage of $\mathrm{c}-\mathrm{C}_{4} \mathrm{~F}_{8} / \mathrm{N}_{2}$ gas mixture was measured under different pressure, different mixing ratio, and different electrode distance. The influence of these three factors on the $\mathrm{PD}$ performance of the gas mixture was obtained and compared with the initial $\mathrm{PD}$ voltage of pure $\mathrm{SF}_{6}$ gas. The results show that the initial $\mathrm{PD}$ voltage of pure $c-\mathrm{C}_{4} \mathrm{~F}_{8}$ gas is 1.3 times that of pure $\mathrm{SF}_{6}$ gas [23]. $\mathrm{c}-\mathrm{C}_{4} \mathrm{~F}_{8}$ and $\mathrm{N}_{2}$ have synergistic effect, and the synergistic coefficient calculated by Takuma's equation under different electrode distance and gas pressure is shown in Table 1.

Yamamoto et al. examined the insulation characteristics of $\mathrm{c}-\mathrm{C}_{4} \mathrm{~F}_{8}$ gas mixtures such as $\mathrm{c}-\mathrm{C}_{4} \mathrm{~F}_{8} / \mathrm{N}_{2}, \mathrm{c}-\mathrm{C}_{4} \mathrm{~F}_{8} /$ air, and $\mathrm{c}-\mathrm{C}_{4} \mathrm{~F}_{8} / \mathrm{CO}_{2}$ under different electric field [24]. The experimental results show that $\mathrm{c}-\mathrm{C}_{4} \mathrm{~F}_{8} / \mathrm{CO}_{2}$ gas mixture has better synergistic effect than the other two mixtures, and improve gas pressure or gap distance can greatly

\begin{tabular}{|c|c|c|c|c|c|}
\hline \multirow[t]{2}{*}{ Electrode distance $/ \mathrm{mm}$} & \multirow{2}{*}{$\begin{array}{c}\text { Gas pressure/ } \\
\text { Mpa }\end{array}$} & \multicolumn{4}{|c|}{$C$} \\
\hline & & $5 \% c-C_{4} F_{8}$ & $10 \% c-C_{4} F_{8}$ & $15 \% c-C_{4} F_{8}$ & $20 \% c-C_{4} F_{8}$ \\
\hline \multirow[t]{2}{*}{10} & 0.20 & 0.48 & 0.50 & 0.54 & 0.53 \\
\hline & 0.25 & 0.20 & 0.40 & 0.44 & 0.63 \\
\hline \multirow[t]{2}{*}{20} & 0.20 & 0.47 & 0.86 & 0.45 & 0.52 \\
\hline & 0.25 & 0.34 & 0.50 & 0.49 & 0.54 \\
\hline \multirow[t]{2}{*}{30} & 0.20 & 0.15 & 0.25 & 0.30 & 0.36 \\
\hline & 0.25 & 0.24 & 0.32 & 0.34 & 0.41 \\
\hline
\end{tabular}

Table 1.

Synergistic coefficient for $c-C_{4} F_{8} / N_{2}$. 


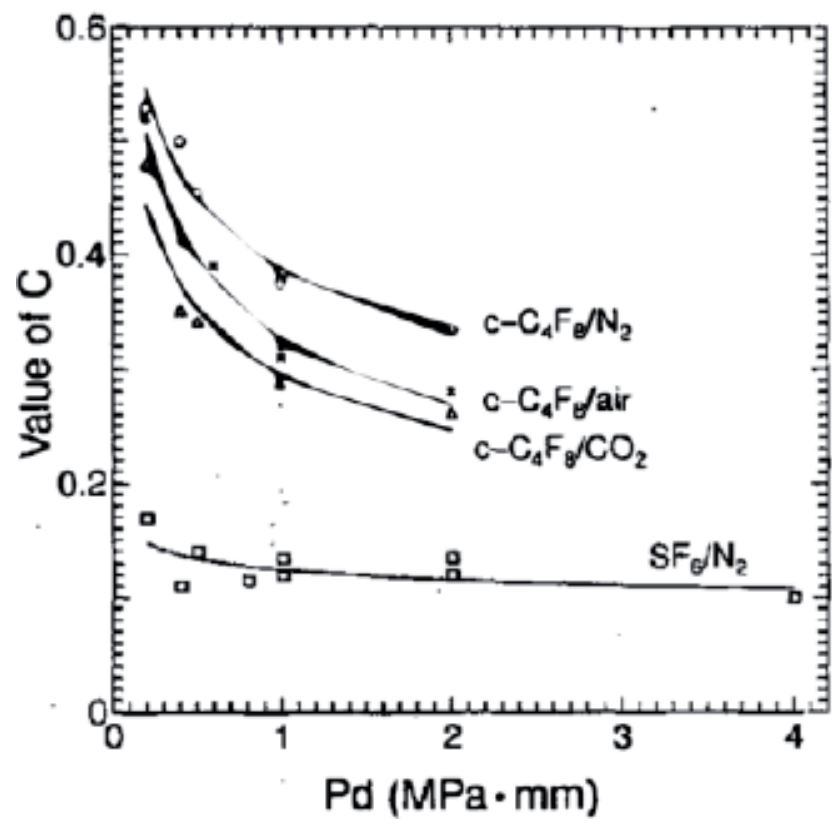

Figure 13.

Relationship of synergistic effect coefficient $\mathrm{C}$ with $\mathrm{Pd}$.

enhance the synergistic effect of $\mathrm{c}-\mathrm{C}_{4} \mathrm{~F}_{8}$ gas mixture. The relationship of synergistic effect coefficient $C$ with $\mathrm{Pd}$ is shown in Figure 13.

\section{$3.2 \mathrm{CF}_{3} \mathrm{I}$}

$\mathrm{CF}_{3} \mathrm{I}$ gas is odorless, nonflammable, chemically stable, and material compatible. The molecular structure of $\mathrm{CF}_{3} \mathrm{I}$ is shown in Figure 14. $\mathrm{CF}_{3} \mathrm{I}$ is considered as one of the ideal alternatives to conventional Freon refrigerant. In terms of environmental characteristics, $\mathrm{CF}_{3} \mathrm{I}$ is an extremely environmental friendly gas, and its GWP value is about $1-5$, much lower than $\mathrm{SF}_{6}$ gas. At the same time, the C-I chemical bond in the molecular structure of $\mathrm{CF}_{3} \mathrm{I}$ is easy to photolysis under solar radiation, resulting in the very short existence time of $\mathrm{CF}_{3} \mathrm{I}$ in the atmosphere, and the ozone destruction potential of the gas can also be ignored. The boiling point of $\mathrm{CF}_{3} \mathrm{I}$ at normal pressure is $-22.5^{\circ} \mathrm{C}$, which indicates that $\mathrm{CF}_{3} \mathrm{I}$ gas will transform from gaseous to liquid when the temperature is lower than $-22.5^{\circ} \mathrm{C}$ [25]. Therefore, $\mathrm{CF}_{3} \mathrm{I}$ gas must be mixed with buffer gas before it can be used in power equipment.

Urquijo et al. experimentally measured the electron transport parameters of $\mathrm{CF}_{3} \mathrm{I}$ and $\mathrm{CF}_{3} \mathrm{I} / \mathrm{N}_{2}$ gas mixture. They analyzed and studied several parameters including electron drift velocity, diffusion coefficient, electron ionization coefficient, and attachment coefficient by means of pulsed Townsend method. Experimental results show that the critical electric field intensity $(E / N)_{\lim }$ of $\mathrm{CF}_{3} \mathrm{I}$ was 437Td, and the insulation strength of the gas is about 1.2 times that of $\mathrm{SF}_{6}$ gas. When the content of $\mathrm{CF}_{3} \mathrm{I}$ is $70 \%$, the dielectric strength of $\mathrm{CF}_{3} \mathrm{I} / \mathrm{N}_{2}$ gas mixture is basically the same with pure $\mathrm{SF}_{6}$ gas [26]. The insulation characteristic comparison of $\mathrm{CF}_{3} \mathrm{I} / \mathrm{N}_{2}$ and $\mathrm{SF}_{6} / \mathrm{N}_{2}$ gas mixtures IS shown in Figure 15. From the figure it can be seen that $\mathrm{CF}_{3} \mathrm{I} / \mathrm{N}_{2}$ and $\mathrm{SF}_{6} / \mathrm{N}_{2}$ gas mixture both have synergistic effect phenomenon, but the phenomenon of $\mathrm{CF}_{3} \mathrm{I} / \mathrm{N}_{2}$ gas mixture is weak when compared with $\mathrm{SF}_{6} / \mathrm{N}_{2}$.

In addition to $\mathrm{CF}_{3} \mathrm{I} / \mathrm{N}_{2}$ mixture, there is also a synergistic effect of $\mathrm{CF}_{3} \mathrm{I} / \mathrm{CO}_{2}$ mixture. Jiao et al. studied the gas mixture of $\mathrm{CF}_{3} \mathrm{I} / \mathrm{CO}_{2}$ with low content of $\mathrm{CF}_{3} \mathrm{I}$, 


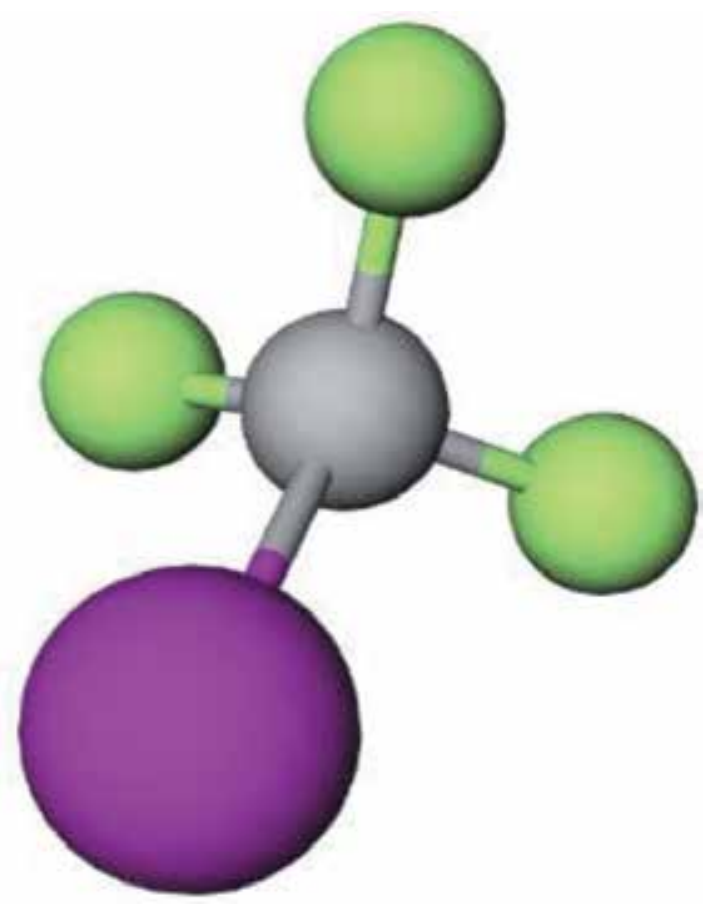

Figure 14 .

Molecular structure of $\mathrm{CF}_{3} I$.

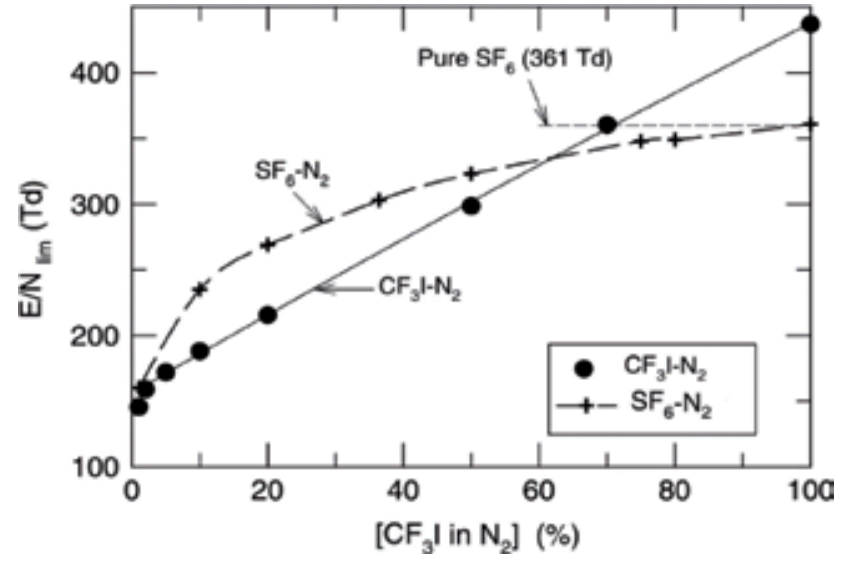

Figure 15.

Comparison of insulation strength between $\mathrm{CF}_{3} I / N_{2}$ and $\mathrm{SF}_{6} / \mathrm{N}_{2}$.

and conducted breakdown test on $\mathrm{CF}_{3} \mathrm{I} / \mathrm{CO}_{2}$ gas mixture with different mixing ratio, different gas pressure, and different discharge gap distance under extremely uneven electric field [27]. The experimental results show that the mixing of trace $\mathrm{CF}_{3} \mathrm{I}$ can significantly increase the breakdown voltage, and the breakdown voltage tends to be stable when the content of $\mathrm{CF}_{3} \mathrm{I}$ gas is over $6 \%$. Although there is still a gap of the breakdown voltage between $\mathrm{CF}_{3} \mathrm{I} / \mathrm{CO}_{2}$ and $\mathrm{SF}_{6}$, the trace amount of $\mathrm{CF}_{3} \mathrm{I}$ has a good synergistic relationship with $\mathrm{CO}_{2}$ mixture. The relationship between breakdown voltage and $\mathrm{CF}_{3} \mathrm{I}$ content under different electrode distances is shown in Figure 16, and the synergistic coefficient of $\mathrm{CF}_{3} \mathrm{I} / \mathrm{CO}_{2}$ gas mixture calculated by Takuma's equation is shown in Table 2 . The author believes that the negative synergetic effect coefficient is caused by the saturation of the breakdown voltage and 


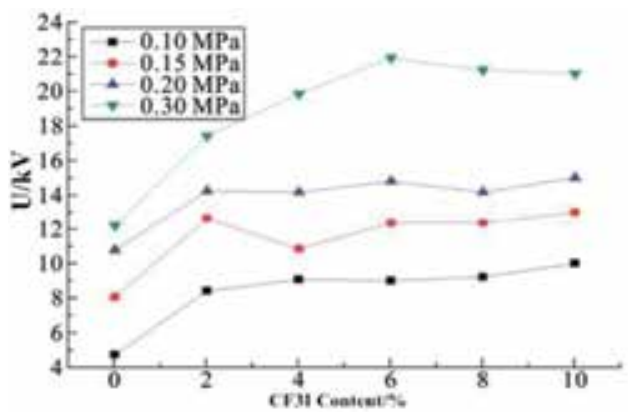

(a)

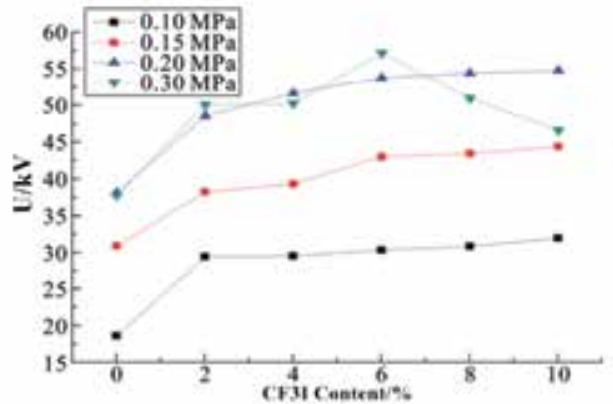

(c)

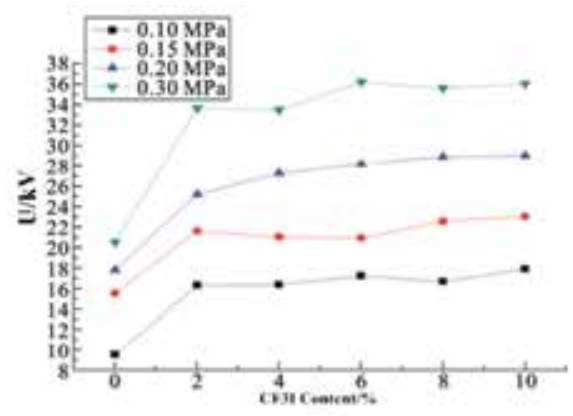

(b)

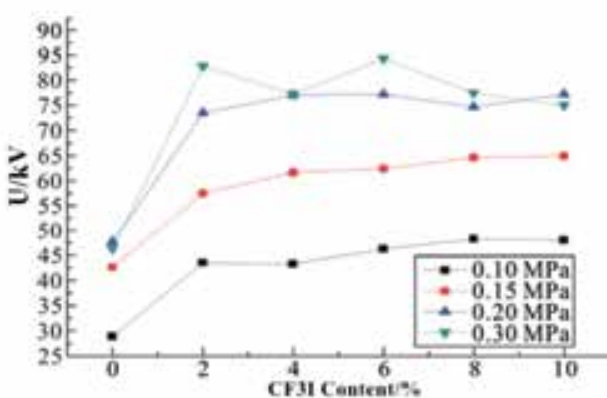

(d)

Figure 16.

Relationship between breakdown voltage and $\mathrm{CF}_{3} I$ content under different electrode distance. (a) $5 \mathrm{~mm}$, (b) $10 \mathrm{~mm}$, (c) $20 \mathrm{~mm}$, (d) $30 \mathrm{~mm}$.

\begin{tabular}{|c|c|c|c|c|c|}
\hline \multirow[t]{2}{*}{ Gap distance /mm } & \multirow[t]{2}{*}{ Pressure /MPa } & \multicolumn{4}{|c|}{ Synergetic coefficient } \\
\hline & & $2 \% \mathrm{CF}_{3} \mathrm{I}$ & $4 \% \mathrm{CF}_{3} \mathrm{I}$ & $6 \% \mathrm{CF}_{3} \mathrm{I}$ & $8 \% \mathrm{CF}_{3} \mathrm{I}$ \\
\hline \multirow[t]{4}{*}{5} & 0.10 & 0.11 & 0.14 & 0.35 & 0.72 \\
\hline & 0.15 & 0.02 & 0.52 & 0.22 & 0.60 \\
\hline & 0.20 & 0.06 & 0.16 & 0.08 & 0.99 \\
\hline & 0.30 & 0.17 & 0.10 & -0.14 & -0.09 \\
\hline \multirow[t]{4}{*}{10} & 0.10 & 0.06 & 0.15 & 0.13 & 0.68 \\
\hline & 0.15 & 0.06 & 0.24 & 0.58 & 0.27 \\
\hline & 0.20 & 0.13 & 0.12 & 0.12 & 0.04 \\
\hline & 0.30 & 0.05 & 0.15 & 0.02 & 0.20 \\
\hline \multirow[t]{4}{*}{20} & 0.10 & 0.06 & 0.15 & 0.22 & 0.36 \\
\hline & 0.15 & 0.21 & 0.40 & 0.18 & 0.31 \\
\hline & 0.20 & 0.15 & 0.16 & 0.10 & 0.04 \\
\hline & 0.30 & -0.07 & -0.20 & -0.82 & -1.32 \\
\hline \multirow[t]{4}{*}{30} & 0.10 & 0.08 & 0.22 & 0.15 & -0.05 \\
\hline & 0.15 & 0.12 & 0.11 & 0.19 & 0.06 \\
\hline & 0.20 & 0.04 & 0.01 & 0.01 & 0.38 \\
\hline & 0.30 & -0.05 & -0.05 & -0.37 & -0.32 \\
\hline
\end{tabular}

Table 2.

Synergetic coefficients of $\mathrm{CF}_{3} \mathrm{I} / \mathrm{CO}_{2}$ gas mixtures using Takuma's equation. 


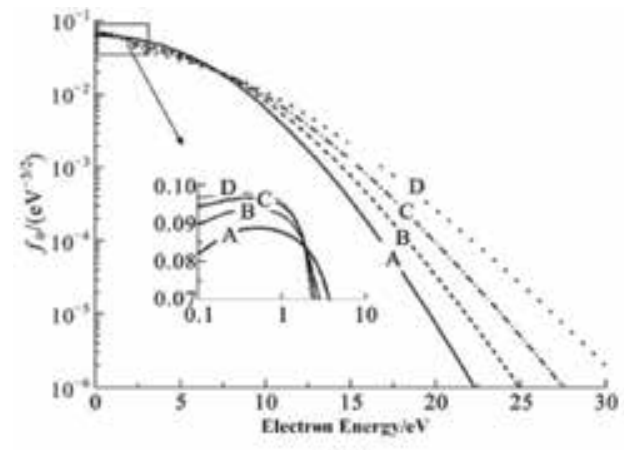

(a)

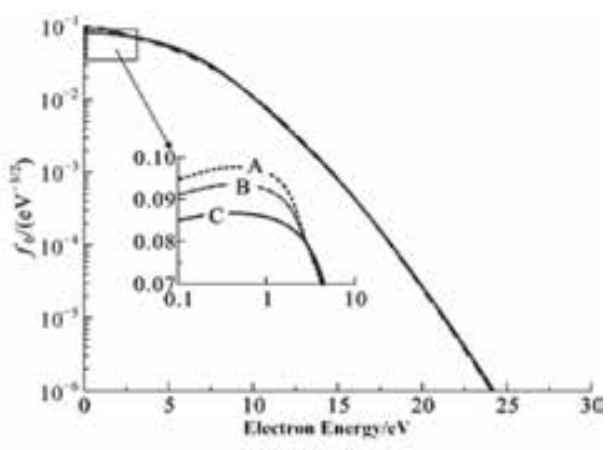

(b)

Figure 17.

EEDF of $\mathrm{CF}_{3}$ I ternary gas mixtures. (a) $\mathrm{CF}_{3} I / S F_{6} / N_{2}$, (b) $C F_{3} I / N_{2} / C F_{4}$.

does not continue to analyze the reason. In fact, this phenomenon may be related to the abnormal breakdown of the gas mixture containing $\mathrm{CO}_{2}$.

In addition to binary $\mathrm{CF}_{3} \mathrm{I}$ gas mixture, some scholars have studied ternary gas mixture containing $\mathrm{CF}_{3} \mathrm{I}$ gas. Xu et al. calculated the electron energy distribution function (EEDF) of $\mathrm{CF}_{3}$ I ternary gas mixtures by solving the Boltzmann equation and propose a new equation to analyze synergistic effect. The results show that if buffer gases such as $\mathrm{N}_{2}$ or $\mathrm{CO}_{2}$ are concluded in the ternary mixture, the distribution of low-energy electrons in EEDF increases, leading to the synergistic effect, but there is no synergistic effect with $\mathrm{CF} 4$. The mixture of two strongly electronegative gases, $\mathrm{CF}_{3} \mathrm{I} / \mathrm{SF}_{6}$, shows weak negative synergistic effect, while the addition of $\mathrm{N}_{2}$ or $\mathrm{CO}_{2}$ can reduce the negative synergistic effect [28]. The EEDF of $\mathrm{CF}_{3} \mathrm{I}$ ternary gas mixtures is shown in Figure 17.

The computational formula of synergistic effect coefficient proposed by $\mathrm{Xu}$ is as follows:

$$
\xi=\frac{(E / N)_{\text {lim,mix }}}{\sum x_{i}(E / N)_{\text {lim,i }}}-1
$$

where $(E / N)_{\text {lim, mix }}$ is the critical reduced field intensity of gas mixture and $(E / N)_{\lim , \mathrm{i}}$ is the critical reduced field intensity of single gas. $x_{i}$ is the molar fraction of the gas component. Fix the buffer mole fraction of gas to 0,10 , and $30 \%$, and change the mixing ratio of $\mathrm{CF}_{3} \mathrm{I}$ and $\mathrm{SF}_{6}$ gas; the $(E / N)_{\text {lim }}$ and synergistic effect coefficient of $\mathrm{CF}_{3} \mathrm{I}$ ternary gas mixtures are shown in Figure 18.

\section{$3.3 \mathrm{C}_{4} \mathrm{~F}_{7} \mathrm{~N}$}

$\mathrm{C}_{4} \mathrm{~F}_{7} \mathrm{~N}$ is an $\mathrm{SF}_{6}$ alternative gas jointly developed by Alstom of France and $3 \mathrm{M}$ of the United States. The commodity name of this gas is Novec 4710 , which is an organic compound containing four $\mathrm{C}$ atoms and seven $\mathrm{F}$ atoms. Its molecular structure is shown in Figure 19. The chemical characteristics of the gas are similar to those of fluoro organic gas, and the chemical characteristics are relatively stable, which can achieve good compatibility with other materials in electrical equipment. The relative molecular weight of $\mathrm{C}_{4} \mathrm{~F}_{7} \mathrm{~N}$ is 195.0 , and it also has a high liquefaction temperature, which is $-4.7^{\circ} \mathrm{C}$ [29]. Therefore, it also needs to be mixed with buffer gas.

$\mathrm{C}_{4} \mathrm{~F}_{7} \mathrm{~N}$ gas mixture is a popular alternative to $\mathrm{SF}_{6}$. At present, most researches on $\mathrm{SF}_{6}$ alternative gases are concentrated on this gas. In order to obtain the optimal buffer gas type and mixing ratio in $\mathrm{C}_{4} \mathrm{~F}_{7} \mathrm{~N}$ gas mixture, $\mathrm{Hu}$ et al. studied the power frequency breakdown performance and synergistic characteristics of $\mathrm{C}_{4} \mathrm{~F}_{7} \mathrm{~N} / \mathrm{CO}_{2}$ and $\mathrm{C}_{4} \mathrm{~F}_{7} \mathrm{~N} / \mathrm{N}_{2}$ gas mixture with uniform electric field [30]. The gas pressure is 

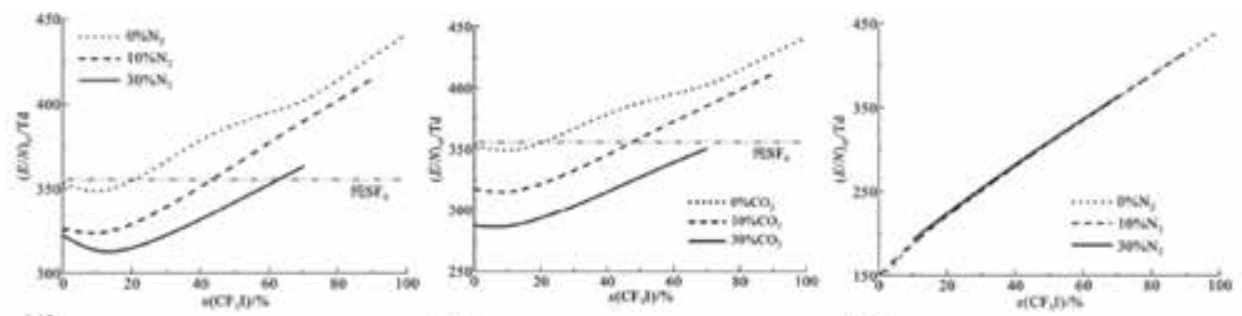

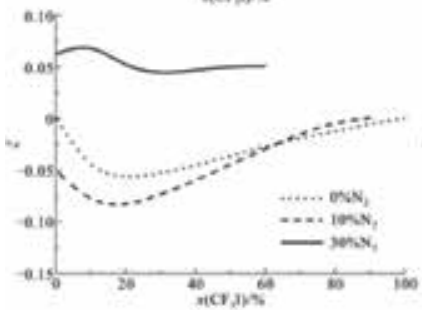

(a)

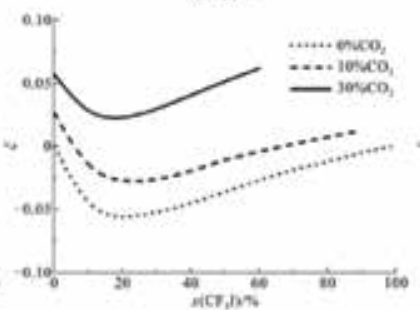

(b)

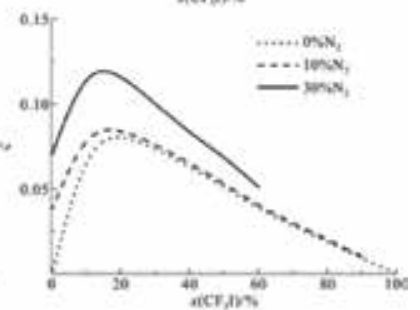

(c)

Figure 18.

The $(\mathrm{E} / \mathrm{N})_{\text {lim }}$ and synergistic coefficient of $C F_{3} I$ gas mixtures. (a) $C F_{3} I / S F_{6} / N_{2},(b) C F_{3} I / S F_{6} / C O_{2}$, (c) $C F_{3} I /$ $\mathrm{CF}_{4} / \mathrm{N}_{2}$.

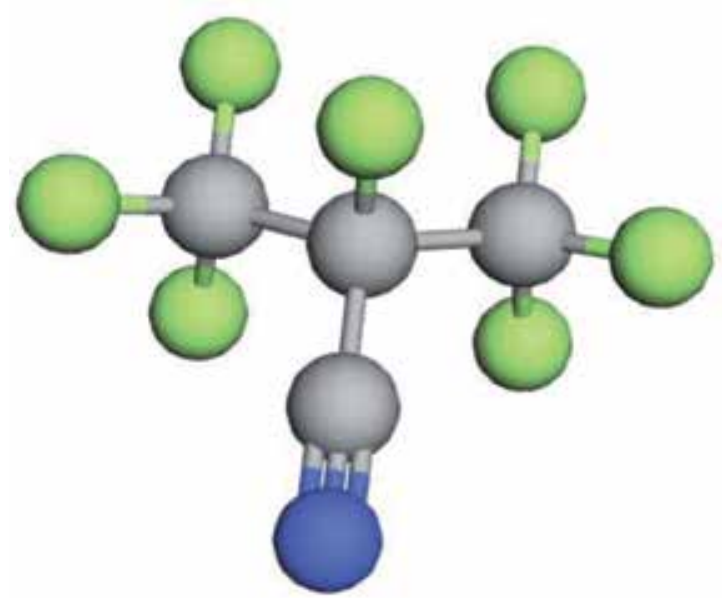

Figure 19.

Molecular structure of $\mathrm{C}_{4} F_{7} N$.

0.1-0.7 MPa, and the $\mathrm{C}_{4} \mathrm{~F}_{7} \mathrm{~N}$ content ratio in gas mixture is $5-20 \%$. The experimental and calculation results show that the $\mathrm{C}_{4} \mathrm{~F}_{7} \mathrm{~N} / \mathrm{CO}_{2}$ and $\mathrm{C}_{4} \mathrm{~F}_{7} \mathrm{~N} / \mathrm{N}_{2}$ gas mixture both have synergistic effects, and the synergistic effect of $\mathrm{C}_{4} \mathrm{~F}_{7} \mathrm{~N} / \mathrm{CO}_{2}$ is stronger than $\mathrm{C}_{4} \mathrm{~F}_{7} \mathrm{~N} / \mathrm{N}_{2}$. The interaction between $\mathrm{C}_{4} \mathrm{~F}_{7} \mathrm{~N}$ and $\mathrm{CO}_{2}$ bimolecular is stronger than that of $\mathrm{C}_{4} \mathrm{~F}_{7} \mathrm{~N}$ and $\mathrm{N}_{2}$, and the research indicates that there is a certain correlation between the synergistic effect of $\mathrm{C}_{4} \mathrm{~F}_{7} \mathrm{~N}$ gas mixture and the intermolecular interaction. This result presents the theoretical calculation and analysis direction for the qualitative judgment of the synergistic effect of $\mathrm{C}_{4} \mathrm{~F}_{7} \mathrm{~N}$ gas mixture. The synergistic coefficient $h$ of $\mathrm{C}_{4} \mathrm{~F}_{7} \mathrm{~N} / \mathrm{CO}_{2}$ and $\mathrm{C}_{4} \mathrm{~F}_{7} \mathrm{~N} / \mathrm{N}_{2}$ calculated by Guo Can's equation is shown in Table 3.

Zheng et al. also studied the synergistic effect and insulation performance of $\mathrm{C}_{4} \mathrm{~F}_{7} \mathrm{~N} / \mathrm{CO}_{2}$ gas mixture and found that the synergistic coefficient was related to avalanche parameters of pure gas. They proposed a graphical method based on the Wieland approximation to calculate the critical electric field of gas mixture. 
Research Progress on Synergistic Effect between Insulation Gas Mixtures DOI: $h$ ttp://dx.doi.org/10.5772/intechopen.90705

\begin{tabular}{|c|c|c|c|c|c|}
\hline \multirow[t]{2}{*}{ Gas type } & \multirow[t]{2}{*}{ Pressure /MPa } & \multicolumn{4}{|c|}{ Coefficient $h$} \\
\hline & & $5 \% \mathrm{C}_{4} \mathrm{~F}_{7} \mathrm{~N}$ & $7 \% \mathrm{C}_{4} \mathrm{~F}_{7} \mathrm{~N}$ & $9 \% \mathrm{C}_{4} \mathrm{~F}_{7} \mathrm{~N}$ & $13 \% \mathrm{C}_{4} \mathrm{~F}_{7} \mathrm{~N}$ \\
\hline \multirow[t]{7}{*}{$\mathrm{C}_{4} \mathrm{~F}_{7} \mathrm{~N} / \mathrm{CO}_{2}$} & 0.1 & 0.57 & 0.63 & 0.69 & 0.77 \\
\hline & 0.2 & 0.49 & 0.59 & 0.63 & 0.69 \\
\hline & 0.3 & 0.46 & 0.52 & 0.57 & 0.69 \\
\hline & 0.4 & 0.45 & 0.53 & 0.57 & 0.69 \\
\hline & 0.5 & 0.45 & 0.54 & 0.59 & 0.73 \\
\hline & 0.6 & 0.47 & 0.55 & 0.62 & 0.75 \\
\hline & 0.7 & 0.50 & 0.58 & 0.64 & 0.76 \\
\hline \multirow[t]{7}{*}{$\mathrm{C}_{4} \mathrm{~F}_{7} \mathrm{~N} / \mathrm{N}_{2}$} & 0.1 & 0.40 & 0.46 & 0.53 & 0.62 \\
\hline & 0.2 & 0.44 & 0.51 & 0.57 & 0.63 \\
\hline & 0.3 & 0.34 & 0.41 & 0.48 & 0.56 \\
\hline & 0.4 & 0.35 & 0.42 & 0.48 & 0.56 \\
\hline & 0.5 & 0.37 & 0.43 & 0.48 & 0.56 \\
\hline & 0.6 & 0.39 & 0.46 & 0.50 & 0.64 \\
\hline & 0.7 & 0.44 & 0.52 & 0.58 & 0.64 \\
\hline
\end{tabular}

Table 3.

Synergistic coefficient $\mathrm{h}$ of $\mathrm{C}_{4} \mathrm{~F}_{7} \mathrm{~N} / \mathrm{CO}_{2}$ and $\mathrm{C}_{4} \mathrm{~F}_{7} \mathrm{~N} / \mathrm{N}_{2}$ gas mixture.

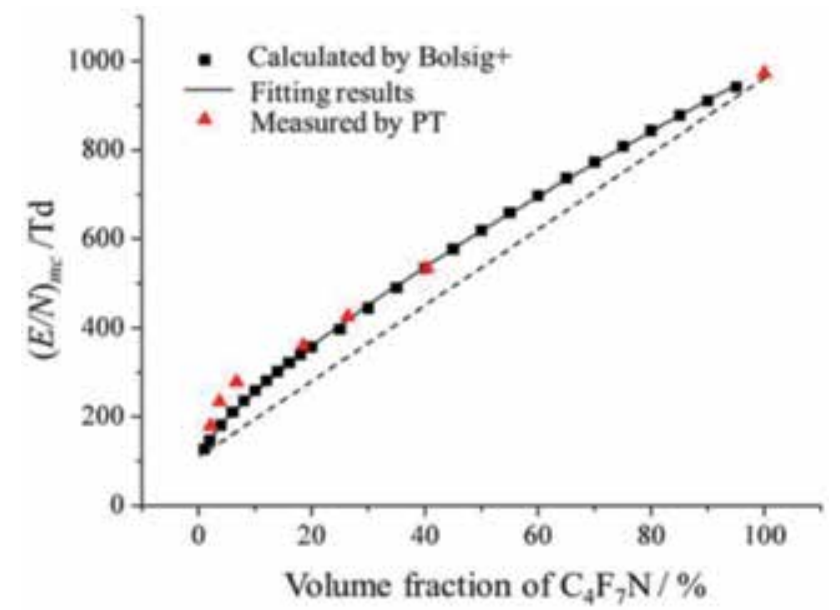

Figure 20.

The relationship of the critical electric field of gas mixture and $\mathrm{C}_{4} \mathrm{~F}_{7} \mathrm{~N}$ content.

Based on the formula, the $\mathrm{C}_{4} \mathrm{~F}_{7} \mathrm{~N}$ content takes $9 \%$ in gas mixture tends to be the optimal mixing ratio [31]. Figure 20 shows the relationship of the critical electric field of gas mixture and $\mathrm{C}_{4} \mathrm{~F}_{7} \mathrm{~N}$ content.

\section{Conclusions}

With the development of the power system, gas-insulated equipment such as GIS will be more and more widely used. At present, $\mathrm{SF}_{6}$ gas is still the most used insulating gas in power systems. However, due to the greenhouse effect of $\mathrm{SF}_{6}$ gas and the 
consensus of countries on the development of low-carbon clean energy systems, it is imperative to use new environmental friendly insulating gas in power equipment. Looking for $\mathrm{SF}_{6}$ replacement gas will also be a continuing research hotspot in the field of electrical engineering. According to the current research status, the replacement of $\mathrm{SF}_{6}$ by single gas and its application in gas insulation equipment is still in the laboratory research stage. The use of an electronegative gas mixed with a buffer gas is a well-feasible solution. This paper reviews the research status of the synergistic effect of insulating gas mixtures including $\mathrm{SF}_{6}$ gas. It has been found that some achievements have been made in the process and mechanism of synergistic effect, the types of synergistic effects under different conditions, and their influencing factors:

1. $\mathrm{SF}_{6}, \mathrm{c}-\mathrm{C}_{4} \mathrm{~F}_{8}, \mathrm{CF}_{3} \mathrm{I}$, and $\mathrm{C}_{4} \mathrm{~F}_{7} \mathrm{~N}$ gas all have synergistic effects when mixed with various buffer gases. $\mathrm{SF}_{6} / \mathrm{N}_{2}$ gas mixture will have negative synergistic effect under the lightning impulse. The synergistic effect of the gas mixture composed of $\mathrm{c}-\mathrm{C}_{4} \mathrm{~F}_{8}, \mathrm{CF}_{3} \mathrm{I}$ and $\mathrm{C}_{4} \mathrm{~F}_{7} \mathrm{~N}$ and $\mathrm{CO}_{2}$ is more significant than that after mixing with $\mathrm{N}_{2}$.

2. The synergistic effect of gas mixture is affected by the type of gas and external conditions such as gas pressure, electric field type, voltage type, etc. The synergistic effect of the same gas mixture may change with the change of external conditions, such as from synergistic effect to negative synergistic or from negative synergistic to synergistic effect.

3. The synergistic effect of the gas mixture has a certain relationship with the electronic transport parameters. However, since the assumptions of the parameter calculation differ greatly from the external conditions of the experimental measurements, the interpretation of the synergistic effect is not universal.

4. The synergistic effect of gas mixture has a relationship with the discharge pattern. When synergistic effect occurs, the initial discharge voltage of the streamer discharge rises. When negative synergistic phenomenon occurs, the streamer discharge gradually changes to leader discharge, and the discharge starting voltage decreases.

\title{
Author details
}

\author{
Su Zhao and Dengming Xiao* \\ Department of Electrical Engineering, Shanghai Jiao Tong University, Shanghai, \\ China
}

*Address all correspondence to: dmxiao@sjtu.edu.cn

\section{IntechOpen}

(C) 2020 The Author(s). Licensee IntechOpen. This chapter is distributed under the terms of the Creative Commons Attribution License (http://creativecommons.org/licenses/ by/3.0), which permits unrestricted use, distribution, and reproduction in any medium, provided the original work is properly cited. (cc) BY 


\section{References}

[1] Koch H, Hopkins M. Overview of gas insulated lines (GIL). In: IEEE Power Engineering Society General Meeting. 2005. pp. 940-944

[2] Yunkun D. Basic Research of the Environmentally Friendly Insulating Gas $\mathrm{CF}_{3} \mathrm{I}$ for its Application in Electric Power Apparatus [PhD Thesis]. Shanghai: Shanghai Jiao Tong University; 2016

[3] Hertz W, Motschmann H, Wittel H. Investigations of the properties of $\mathrm{SF}_{6}$ as an arc quenching medium. Proceedings of the IEEE. 1971;59(4):485-492

[4] Malik NH, Qureshi AH, Theophilus GD, et al. Prebreakdown phenomenon in SF6-N2 mixtures at various pressures. Electrical insulation. In: 1978 IEEE International Conference on Electrical Insulation. 1978. pp. 140-145

[5] Abdelsalam M, Abdellah M. Dielectric strength of compressed insulating gases and their mixtures. IEEE Transactions on Industry Applications. 1978;IA-14(6):516-522

[6] Wootton RE, Chantry PJ. A critique of methods for calculating the dielectric strength of gas mixtures and a proposed test for, $\eta$-synergism. In: Proceedings of the Second International Symposium on Gaseous Dielectrics. Knoxville, USA; 1980. pp. 32-42

[7] Kline LE, Davies D, Chen C, et al. Dielectric properties for $\mathrm{SF}_{6}$ and $\mathrm{SF}_{6}$ mixtures predicted from basic data. Journal of Applied Physics. 1979;50(11):6789-6796

[8] Zhao S, Xiao D, Xue P, et al. Analysis of insulation performance and polar effect of $\mathrm{CF}_{3} \mathrm{I} / \mathrm{CO}_{2}$ mixtures. IEEE Transactions on Dielectrics and Electrical Insulation. 2018;25(4):1364-1370

[9] Ravishankara AR, Solomon S, Turnipseed AA, et al. Atmospheric lifetimes of long-lived halogenated species. Science. 1993;259(5092):194-199

[10] Rinsland CP, Brown LR, Farmer CB. Infrared spectroscopic detection of sulfur-hexafluoride $\left(\mathrm{SF}_{6}\right)$ in the lower stratosphere and upper troposphere. Journal of Geophysical Research: Atmospheres. 1990;95(D5):5577-5585

[11] Reilly J, Prinn R, Harnisch J, et al. Multi-gas assessment of the Kyoto protocol. Nature. 1999;401(6753):549

[12] Chantry PJ, Wootton RE. A critique of methods for calculating the dielectric strength of gas mixtures. Journal of Applied Physics. 1981;52(4):2731-2739

[13] Christophorou LG, James DR, Mathis RA. Dielectric gas mixtures with polar components. Journal of Physics D: Applied Physics. 1981;14(4):675

[14] Okubo H, Hayakawa N. Dielectric characteristics and electrical insulation design techniques of gases and gas mixtures as alternatives to $\mathrm{SF}_{6}$. In: Gaseous Dielectrics X. Springer; 2004. pp. 243-252

[15] Yamada T, Ishida T, Hayakawa N, et al. Partial discharge and breakdown mechanisms in ultra-dilute $\mathrm{SF}_{6}$ and $\mathrm{PFC}$ gases with $\mathrm{N}_{2}$ gas. Journal of Physics $\mathrm{D}$ : Applied Physics. 2002;35(21):2760-2765

[16] Osmokrovic P, Stojkanovic M, Stankovic K, et al. Synergistic effect of $\mathrm{SF}_{6}$ and $\mathrm{N}_{2}$ gas mixtures on the dynamics of electrical breakdown. IEEE Transactions on Dielectrics and Electrical Insulation. 2012;19(2):677-688

[17] Hayakawa N, Yoshitake Y, Koshino N, et al. Impulse partial discharge characteristics and their mechanisms under non-uniform 
electric field in $\mathrm{N}_{2} / \mathrm{SF}_{6}$ gas mixtures. IEEE Transactions on Dielectrics and Electrical Insulation.

2005;12(5):1035-1042

[18] Chen Q, Xiao D, Qiu Y. Discharge characteristics of $\mathrm{SF}_{6} / \mathrm{N}_{2}$ gas mixture. Journal of Xian Jiaotong University. 2001;35(4):338-342

[19] Nakanishi K. New gaseous insulation. IEEE Transactions on Electrical Insulation. 1986;EI-21(6): 933-937

[20] Takuma T, Watanabe T, Kita K. Breakdown characteristics of compressed-gas mixtures in nearly uniform fields. Proceedings of the Institution of Electrical Engineers. 1972;119(7):927-928

[21] Guo C, Zhang Q, Ma J, et al. Negative synergistic effect of $\mathrm{SF}_{6} / \mathrm{N}_{2}$ gas mixtures in non-uniform electric field under lightning impulse. IEEE Transactions on Dielectrics and Electrical Insulation. 2018;25(4):1393-1402

[22] Zhao S, Jiao J, Zhao X, et al. Synergistic effect of c-C4F8/N2 gas mixtures in slightly non-uniform electric field under lightning impulse. In: IEEE Electrical Insulation Conference. IEEE; 2016. pp. 531-534

[23] Xing W, Zhang G, Li K, et al. Experimental study of partial discharge characteristics of $\mathrm{C}_{4} \mathrm{~F}_{8} / \mathrm{N}_{2}$ mixtures. Proceeding of the CSEE. 2011;31(7):119-124

[24] Yamamoto O, Takuma T, Hamada $\mathrm{H}$, et al. Applying a gas mixture containing $\mathrm{c}-\mathrm{C}_{4} \mathrm{~F}_{8}$ as an insulation medium. IEEE Transactions on Dielectrics and Electrical Insulation. 2001;8(6):1075-1081

[25] Zhao S, Xiao D, Xue P, et al. Experimental research on polarity effect of $\mathrm{CF}_{3} \mathrm{I} / \mathrm{N}_{2}$ mixtures under lightning impulse. IEEE Transactions on Dielectrics and Electrical Insulation. 2018;25(4):1357-1362

[26] De Urquijo J, Juárez AM, Basurto E, et al. Electron impact ionization and attachment, drift velocities and longitudinal diffusion in $\mathrm{CF}_{3} \mathrm{I}$ and $\mathrm{CF}_{3} \mathrm{I}-\mathrm{N}_{2}$ mixtures. Journal of Physics D: Applied Physics. 2007;40(7):2205

[27] Jiao J, Xiao D, Zhao S, et al. Insulation characteristics for $\mathrm{CF}_{3} \mathrm{I}-\mathrm{CO}_{2}$ gas mixtures with low proportion of $\mathrm{CF}_{3} \mathrm{I}$ in extreme non-uniform electric field. High Voltage Engineering. 2017;43(3):772-779

[28] Xu L, Cheng L, Du W, et al. Analysis of the insulation characteristic of $\mathrm{CF}_{3} \mathrm{I}$ ternary gas mixture using Boltzmann equation. High Voltage Engineering. 2017;43(3):721-726

[29] Li Y, Zhang X, Xiao S, et al.

Decomposition properties of $\mathrm{C}_{4} \mathrm{~F}_{7} \mathrm{~N} /$ $\mathrm{N}_{2}$ gas mixture: An environmentally friendly gas to replace $\mathrm{SF}_{6}$. Industrial \& Engineering Chemistry Research. 2018;57(14):5173-5182

[30] Hu S, Zhou W, Zheng Y, et al. Power Frequency Breakdown Experiments and Synergistic Effect Analysis of $\mathrm{C}_{4} \mathrm{~F}_{7} \mathrm{~N} /$ $\mathrm{CO}_{2}$ and $\mathrm{C}_{4} \mathrm{~F}_{7} \mathrm{~N} / \mathrm{N}_{2}$ Mixtures. High Voltage Engineering. In press

[31] Zheng Y, Yan X, Chen W, et al. Calculation of electrical insulation of $\mathrm{C}_{4} \mathrm{~F}_{7} \mathrm{~N} / \mathrm{CO}_{2}$ mixed gas by avalanche characteristics of pure gas. Plasma Research Express. 2019:025013 



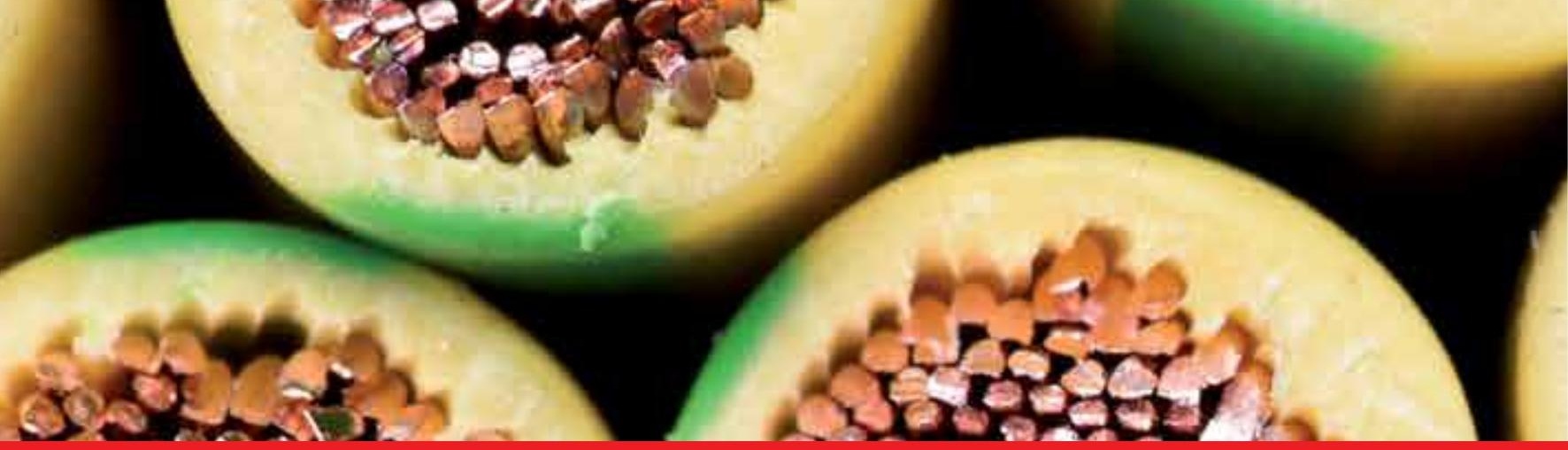

\section{Edited by Dengming Xiao and Krishnaswamy Sankaran}

Electrostatics and dielectric materials have important applications in modern society. As such, they require improved characteristics. More and more equipment needs to operate at high frequency, high voltage, high temperature, and other harsh conditions. This book presents an overview of modern applications of electrostatics and dielectrics as well as research progress in the field.

\section{IntechOpen}
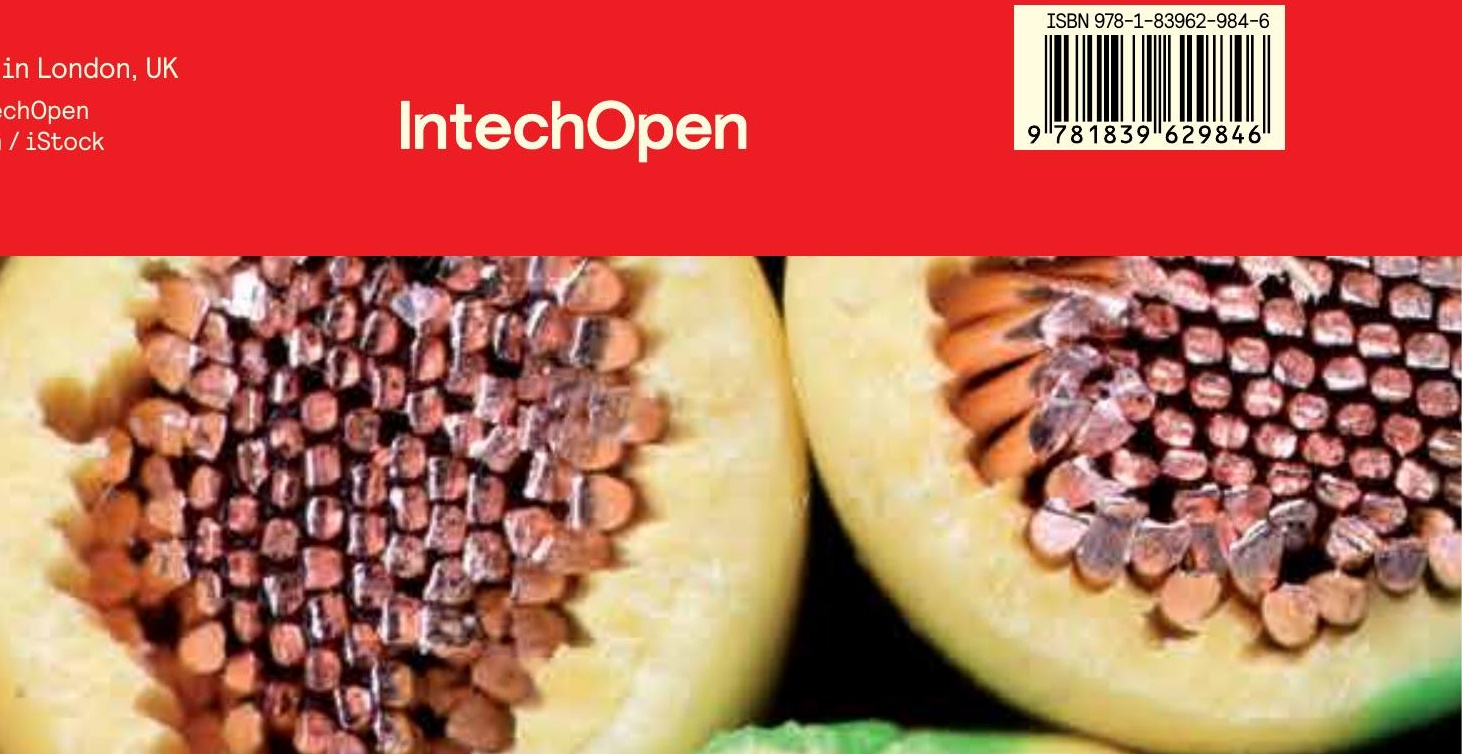